University of Tennessee Health Science Center

UTHSC Digital Commons

\title{
An Interface of the Taste and Reward Systems in the Brainstem and Its Role in Feeding
}

Louis Saites

University of Tennessee Health Science Center

Follow this and additional works at: https://dc.uthsc.edu/dissertations

Part of the Biochemical Phenomena, Metabolism, and Nutrition Commons, Medical Biochemistry Commons, and the Neurosciences Commons

\section{Recommended Citation}

Saites, Louis (http://orcid.org/0000-0002-7124-9642), "An Interface of the Taste and Reward Systems in the Brainstem and Its Role in Feeding" (2018). Theses and Dissertations (ETD). Paper 462.

http://dx.doi.org/10.21007/etd.cghs.201.0456.

This Dissertation is brought to you for free and open access by the College of Graduate Health Sciences at UTHSC Digital Commons. It has been accepted for inclusion in Theses and Dissertations (ETD) by an authorized administrator of UTHSC Digital Commons. For more information, please contact jwelch30@uthsc.edu. 


\title{
An Interface of the Taste and Reward Systems in the Brainstem and Its Role in Feeding
}

\author{
Abstract \\ We eat what tastes good. We also eat because it is necessary for our health. In fact, some of the most \\ nutritious foods (e.g., vegetables) are often less appetizing, and the tastiest (e.g., fast food, ice cream) \\ may be the least healthy. Despite the former, we may also have a lower limit of what we accept at which \\ point nutrition becomes irrelevant (e.g., "spinach is just too yucky"). Further, we may eat unhealthily \\ because of overwhelming urges. We investigated the complex interactions of taste and feeding at the \\ neurobiological level using the experiments described.
}

In one sense, this neurobiology begins at the periphery with information about ingested substances (i.e., presumably food) being sent to central nuclei. The taste pathways provide one of these routes to the central nervous system. In terms of regulating feeding, we have the neurobiological substrates for urge, pleasure, and displeasure. The relationship of the dopamine (DA) system with reward is well-known, and indeed, studies have shown taste nuclei project to these areas.

Since earlier studies and data collected in our lab showed that the neurons of the parabrachial nucleus (PBN) projected to the ventral tegmental area (VTA), and lesioning the PBN attenuates taste-elicited release of DA in the nucleus accumbens, we hypothesized this connection plays a crucial role in the control of feeding, especially with regard to the processing of both appetitive and aversive stimuli, and the relationship of this processing to classical reward circuitry. We therefore utilized a number of neuroanatomical and behavioral techniques to probe taste and intake-related activity in the PBN, VTA, and the PBN-to-VTA circuit. The overarching goal was to contribute to a comprehensive understanding of the taste and reward neural mechanisms that mediate feeding.

We used a variety of immunohistochemical methods to test our hypotheses, including one measuring cFos-like immunoreactivity (FLI) in neurons (a measure that correlates with neuronal activation in some systems such as taste). Intraoral stimuli increased FLI in the PBN across a number of subnuclei, and in this case, we used a diaminobenzidine stain (DAB) with brightfield microscopy. Comparing C57BL6/J (B6) with mice lacking TRPM5 (KO) showed that some of this increase is driven by taste receptor input, but this effect is predominantly for quinine hydrochloride $(\mathrm{QHCl})$. On the other hand, increases in FLI to sucrose (relative to water) in the lateral PBN were the same for both $\mathrm{B} 6$ and $\mathrm{KO}$ mice, leading to the conclusion that this FLI may be visceral in nature. Sucrose-elicited FLI in the external lateral subnucleus (el) was probably visceral, whereas QHCl-elicited FLI there was taste-related. We also combined measurement of FLI with retrograde tracing under fluorescent microscopy to compare activity in PBN projections to the VTA and gustatory thalamus (VPMpc). Retrograde tracing revealed two largely independent projections, with VTA-projecting neurons found more contralaterally, and VPMpc-projecting neurons found ipsilaterally. However, both types of cells are found in the caudal, gustatory "waist" portion of the PBN. Interestingly, there is a lack of VTA-projecting cells in the el. Patterns of FLI were consistent with the DAB

experiment, except with higher expression as compared to water in this fluorescent experiment in a few subnuclei. This may have been due to methodological differences. As for double-labeled cells, more VTAprojecting cells expressed FLI in response to sucrose or QHCl than to water; this numbered to only about $5 \%$ of cells, however, and did not differ according to side. This was compared to double-labeling in VPMpc- projecting cells, where the percent of tracer was around 10\% for both $\mathrm{QHCl}$ and sucrose on the ipsilateral side and $5 \%$ on the contralateral side.

We looked at FLI throughout the VTA as well to see if the activity indicated there was a differential 
response to stimuli with varying taste valence. First, using the same intraorally-stimulated mice with DABstained sections, we observed FLI in the VTA. It did not occur in a stimulus-specific fashion and apparently not in a taste-dependent fashion (no significant differences between B6 and KO). In another experiment using fluorescent stains and confocal microscopy, we looked at the FLI in the VTA while delineating it by subnuclei, counting section by section, and identifying DA and GABA cell types. There were many more DA cells in the VTA than GABA cells, and they had distinct patterns of expression across subnuclei and section levels (i.e., within the anteroposterior [AP] dimension). The rostromedial tegmental area was located as a region with higher GABA cell expression. More DA cells were double-labeled with $\mathrm{FLI}$ for $\mathrm{QHCl}$ than for water or sucrose in the caudal linear nucleus of the raphe. Few GABA cells were double-labeled with FLI.

To show the PBN-to-VTA circuit's role in taste-mediated feeding, we attempted a procedure that would selectively activate VTA-projecting PBN neurons using designer receptors exclusively activated by designer drugs (DREADDs). However, we were unable to verify the efficacy of clozapine-N-oxide to activate the circuit and opted for an alternative manipulation. We instead inhibited the VTA with direct injections of the GABA agonist, muscimol. This resulted in mice reducing their licking (relative to baseline) of sucralose, but not $\mathrm{QHCl}$ or water (i.e., an arrangement of non-caloric stimuli with palatable, aversive, and neutral valence). Muscimol also reduced licking of sucrose and QHCl-adulterated sucrose (i.e., caloric stimuli). The reduction in licking to caloric stimuli was accompanied by a decrease in the rate of intake, i.e., muscimol-inhibited mice slowed their lick rate and possibly stopped licking sooner compared to vehicle- injected controls.

Overall, this project confirmed that both the PBN and VTA function to communicate taste and reward information. Although the PBN-to-VTA circuit's function remained elusive, the evidence of the direct path connecting these two nuclei was fortified. Further, to our knowledge, this was the first time evidence was found of its existence as a PBN projection pathway that is mostly separate from the projection to the gustatory thalamus. Combined with the knowledge of this circuit, the activity in these nuclei and the ability to affect consumption by inactivating the VTA suggest the PBN and VTA work together to influence feeding by detecting and integrating information about palatability and calories.

\section{Document Type}

Dissertation

Degree Name

Doctor of Philosophy (PhD)

\section{Program}

Biomedical Sciences

\section{Research Advisor}

John D. Boughter, Ph.D.

\section{Keywords}

Addiction, Dopamine, Eating, GABA, Gustation, Obesity

\section{Subject Categories}

Biochemical Phenomena, Metabolism, and Nutrition | Medical Biochemistry | Medical Sciences | Medicine and Health Sciences | Neurosciences 
An Interface of the Taste and Reward Systems in the Brainstem and Its Role in Feeding

\author{
A Dissertation \\ Presented for \\ The Graduate Studies Council \\ The University of Tennessee \\ Health Science Center \\ In Partial Fulfillment \\ Of the Requirements for the Degree \\ Doctor of Philosophy \\ From The University of Tennessee
}

By

Louis Saites

August 2018 
Copyright (C) 2018 by Louis Saites. All rights reserved. 


\section{DEDICATION}

To my family and friends. 


\section{ACKNOWLEDGEMENTS}

I start with my mentor, Dr. John Boughter, who welcomed me into his lab as well as his home. I met Dr. Boughter while taking his Behavioral Neuroscience class and enjoyed his enthusiastic approach to teaching. Later, I would come to Dr. Boughter to complete the final official rotation allowed by our graduate program. I was seeking a lab in which to study addiction, and he made it clear that was not something which his lab researched. Still, he agreed to let me rotate. To my delight on the first day of the rotation, I was given a 12-inch stack of articles and told to propose a project studying the overlap of reward (a phenomenon related to addiction) and taste (the broad field Dr. Boughter's lab studies). I happily accepted the challenge and welcomed the opportunity to foster my independence. This rotation assignment launched me into the trek that would culminate into the work presented in this dissertation. As if providing an awesome lab in which to work was not enough, Dr. Boughter cooks a mean Thanksgiving dinner!

This brings me to my committee members: Drs. Kristin Hamre, Bill Armstrong, Matt Ennis, and WenLin Sun. I am grateful to have found such thoughtful and supportive guides. You shared your knowledge and insight. You tested my ability to think critically in ways that I would describe as "tough but fair". My skill in pursuing the use of science has improved as a result of your challenging and inspiring supervision.

I also owe appreciation to my lab mates: Dr. Lianyi Lu, Dr. Vivian Guedes, Dr. Jennifer Saputra, and Stephanie Staszko. First to Dr. Lu, you were here the whole time, maintaining the lab and always willing to help. Thank you for teaching me how to do microinjections and keeping the solution carboys full. Dr. Vivian Guedes, thank you for teaching me immunohistochemistry and offering critical insight. Dr. Jennifer Saputra, I have referenced the protocols in your lab notebook too many times to count. Stephanie Staszko, you arrived as I was halfway through and contributed a few favors as well. Thanks for always being gracious about sharing the Davis rigs.

Beyond the Boughter lab, several others contributed to this work. Dr. Kristen O'Connell provided the two viral vectors I used in the DREADDs experiment (Chapter 8). The expertise of Dr. T. J. Hollingsworth, who had to overhaul the confocal microscope midway through the experiments presented in Chapter 7. I also thank the principal investigator of our lab's closest collaborators, Dr. Max Fletcher and his student, Jordan Ross. Dr. Fletcher, I do not know how Chapter 8 would have turned out had you not in passing suggested I try a pharmacological inhibition of the VTA. I also thank you for the assistance with ordering our viral vectors, teaching me how to implant cannulas in mice brains, and the use of your lab space for some of my last dissertation experiments. Jordan, thank you for teaching me how to use the microsyringe pump and lending me your headscrews.

Finally, to all my family and friends, to whom this dissertation is dedicated, there are so many of you, I dare not begin to try naming, lest I forget and hurt feelings. Instead, please know you all mean so much to me. For all the moments of patience, 
understanding, empathizing. For all the long-talks and adventurous walks. For all the times we shared and times you cared. I sincerely thank you, thank you, thank you. I truly could not have done this without you. 


\begin{abstract}
We eat what tastes good. We also eat because it is necessary for our health. In fact, some of the most nutritious foods (e.g., vegetables) are often less appetizing, and the tastiest (e.g., fast food, ice cream) may be the least healthy. Despite the former, we may also have a lower limit of what we accept at which point nutrition becomes irrelevant (e.g., "spinach is just too yucky"). Further, we may eat unhealthily because of overwhelming urges. We investigated the complex interactions of taste and feeding at the neurobiological level using the experiments described.
\end{abstract}

In one sense, this neurobiology begins at the periphery with information about ingested substances (i.e., presumably food) being sent to central nuclei. The taste pathways provide one of these routes to the central nervous system. In terms of regulating feeding, we have the neurobiological substrates for urge, pleasure, and displeasure. The relationship of the dopamine (DA) system with reward is well-known, and indeed, studies have shown taste nuclei project to these areas.

Since earlier studies and data collected in our lab showed that the neurons of the parabrachial nucleus $(\mathrm{PBN})$ projected to the ventral tegmental area (VTA), and lesioning the PBN attenuates taste-elicited release of DA in the nucleus accumbens, we hypothesized this connection plays a crucial role in the control of feeding, especially with regard to the processing of both appetitive and aversive stimuli, and the relationship of this processing to classical reward circuitry. We therefore utilized a number of neuroanatomical and behavioral techniques to probe taste and intake-related activity in the PBN, VTA, and the PBN-to-VTA circuit. The overarching goal was to contribute to a comprehensive understanding of the taste and reward neural mechanisms that mediate feeding.

We used a variety of immunohistochemical methods to test our hypotheses, including one measuring c-Fos-like immunoreactivity (FLI) in neurons (a measure that correlates with neuronal activation in some systems such as taste). Intraoral stimuli increased FLI in the PBN across a number of subnuclei, and in this case, we used a diaminobenzidine stain (DAB) with brightfield microscopy. Comparing C57BL6/J (B6) with mice lacking TRPM5 (KO) showed that some of this increase is driven by taste receptor input, but this effect is predominantly for quinine hydrochloride $(\mathrm{QHCl})$. On the other hand, increases in FLI to sucrose (relative to water) in the lateral PBN were the same for both B6 and KO mice, leading to the conclusion that this FLI may be visceral in nature. Sucrose-elicited FLI in the external lateral subnucleus (el) was probably visceral, whereas QHCl-elicited FLI there was taste-related. We also combined measurement of FLI with retrograde tracing under fluorescent microscopy to compare activity in PBN projections to the VTA and gustatory thalamus (VPMpc). Retrograde tracing revealed two largely independent projections, with VTA-projecting neurons found more contralaterally, and VPMpc-projecting neurons found ipsilaterally. However, both types of cells are found in the caudal, gustatory "waist" portion of the PBN. Interestingly, there is a lack of VTA-projecting cells in the el. Patterns of FLI were consistent with the DAB 
experiment, except with higher expression as compared to water in this fluorescent experiment in a few subnuclei. This may have been due to methodological differences. As for double-labeled cells, more VTA-projecting cells expressed FLI in response to sucrose or $\mathrm{QHCl}$ than to water; this numbered to only about 5\% of cells, however, and did not differ according to side. This was compared to double-labeling in VPMpcprojecting cells, where the percent of tracer was around 10\% for both $\mathrm{QHCl}$ and sucrose on the ipsilateral side and $5 \%$ on the contralateral side.

We looked at FLI throughout the VTA as well to see if the activity indicated there was a differential response to stimuli with varying taste valence. First, using the same intraorally-stimulated mice with DAB-stained sections, we observed FLI in the VTA. It did not occur in a stimulus-specific fashion and apparently not in a taste-dependent fashion (no significant differences between B6 and KO). In another experiment using fluorescent stains and confocal microscopy, we looked at the FLI in the VTA while delineating it by subnuclei, counting section by section, and identifying DA and GABA cell types. There were many more DA cells in the VTA than GABA cells, and they had distinct patterns of expression across subnuclei and section levels (i.e., within the anteroposterior [AP] dimension). The rostromedial tegmental area was located as a region with higher GABA cell expression. More DA cells were double-labeled with FLI for $\mathrm{QHCl}$ than for water or sucrose in the caudal linear nucleus of the raphe. Few GABA cells were double-labeled with FLI.

To show the PBN-to-VTA circuit's role in taste-mediated feeding, we attempted a procedure that would selectively activate VTA-projecting PBN neurons using designer receptors exclusively activated by designer drugs (DREADDs). However, we were unable to verify the efficacy of clozapine-N-oxide to activate the circuit and opted for an alternative manipulation. We instead inhibited the VTA with direct injections of the GABA agonist, muscimol. This resulted in mice reducing their licking (relative to baseline) of sucralose, but not $\mathrm{QHCl}$ or water (i.e., an arrangement of non-caloric stimuli with palatable, aversive, and neutral valence). Muscimol also reduced licking of sucrose and QHCl-adulterated sucrose (i.e., caloric stimuli). The reduction in licking to caloric stimuli was accompanied by a decrease in the rate of intake, i.e., muscimol-inhibited mice slowed their lick rate and possibly stopped licking sooner compared to vehicleinjected controls.

Overall, this project confirmed that both the PBN and VTA function to communicate taste and reward information. Although the PBN-to-VTA circuit's function remained elusive, the evidence of the direct path connecting these two nuclei was fortified. Further, to our knowledge, this was the first time evidence was found of its existence as a PBN projection pathway that is mostly separate from the projection to the gustatory thalamus. Combined with the knowledge of this circuit, the activity in these nuclei and the ability to affect consumption by inactivating the VTA suggest the PBN and VTA work together to influence feeding by detecting and integrating information about palatability and calories. 


\section{PREFACE}

The studies of this dissertation set out to investigate the phenomenon of why we eat what we eat and test some of the hypotheses derived from this concept by asking specific questions in the category, "What is the underlying neurobiology?" Consider two scenarios that illustrate how the sense of taste interacts with mechanisms for affect and feeding. Both scenarios begin with the organism experiencing the state of hunger and subsequent exposure to appetitive pre-ingestive stimulation (e.g., the organism smells something it identifies as potentially food). The organism will experience an urge to consume the substance and an anticipatory pleasure of alleviating hunger. The point at which the organism causes the substance to enter its oral cavity is where the two scenarios diverge. In the first scenario, the organism experiences a palatable taste. This further elicits pleasure and the urge to swallow. In the second scenario, the organism experiences a strongly aversive taste. This opposes the initial appetitive effects, elicits displeasure, and reverses the directionality of behavior motivated by urge, causing egestion. The neurobiology underlying the markedly differential behavioral responses (and the suspected differences in the mediation of its preceding pre-ingestive taste sensation and affect) in these two scenarios is the subject of this dissertation.

The pleasure and displeasure of food tastes are mediated by the reward system, and dysfunctions within any of the neurobiology for eating may lead to pathological consequences. The ability of addictive drugs to hijack the reward system is well known. Drugs disrupt the normal release of dopamine (DA) in response to cues which typically prime behavior to consume something which will evoke pleasure. However, as tolerance to drugs develops, the pleasure may wane, leaving behind a compulsion to consume despite the elevated cost-to-benefit ratio. Interestingly, some substances we call "food" actually have scant nutritional value yet taste pleasurable. Because of their ability to elicit our affect, these may cause a similar pathological and maladaptive behavioral pattern as drugs. The pleasure derived from the food may lower, but if the drive to consume remains, feeding may continue despite neither pleasure nor nutrition being present. Such a phenomenon blurs the line between drugs and food, but empirical validation of this remains scarce.

The DA system and its mesoaccumbens and nigrostriatal pathways are connected to centers for both taste and feeding. Modulation of DA release likely drives urges to consume palatable food, pleasurable drugs, and to approach their associated cues. Lesioning the parabrachial nucleus (PBN) in the pons of the brainstem disrupts the ability of palatable food to elicit DA release in the nucleus accumbens (NAc). Changes in the expression of $\triangle F$ osB in D1-receptor-expressing GABAergic medium spiny neurons of the NAc - a sign that plasticity potentiating addiction has occurred-has also been shown to change after the consumption of some foods. In contrast, another change in plasticity to palatable foods and addictive drugs actually differentiates these. Similar to cocaine, operant responding procedures used for food or sucrose potentiated glutamatergic synapses in the ventral tegmental area (VTA); however, these effects were shorter, with the ratio of AMPA to NMDA receptors returning to its original expression between 7 
days and 3 weeks after the last session. Even further, habituation of DA release to food rewards occurs in the NAc shell, but not in medial prefrontal cortex and NAc core. Chronic, but not acute morphine, can sensitize the NAc shell so that habituation of DA release to food reward no longer occurs. This may mean that although food itself cannot cause "food addiction" via sensitizing the DA system, the prior use of some addictive substances can.

Paradoxically, feeding behavior can become pathological in either direction. Bulimia nervosa (BN) and anorexia nervosa (AN) occur when someone refuses to allow food to be ingested or absorbed. Both of these are accepted and defined in the 5 th edition of the Diagnostics and Statistics Manual (DSM-V) used by psychiatrists. Conversely, binge eating disorder (BED) is only considered a research category. Despite BED's nascent status, neurobiological content on taste-mediated feeding is more relevant to the pathological effects of overeating; thus, I will briefly direct attention to BED and the effects of long-term overeating.

The first description of BED may have been in the 1990s. The working definition proposed that BED is, "eating an amount of food in a discrete period of time that is definitely larger than most people would eat in a similar period of time under similar circumstances". Over a lifetime, prevalence of BED is estimated at 3.5\% in women and $2.0 \%$ in men. These rates are twice as high as either AN or BN. As might be expected, BED is higher in obese individuals. BED has been proposed to parallel Koob and LeMoal's allostatic model of addiction, and extensive evidence indicates the involvement of the dopaminergic system in the modulation of feeding. No long term, effective treatment for BED has been identified, and BED is associated with obesity and diabetes.

Moreover, obesity and diabetes come with their own pathologies and may result from a lifetime of unhealthy eating habits. Obesity in the United States has skyrocketed in the last couple of decades with more than $35 \%$ of U.S. adults and about $17 \%$ of children and adolescents aged $2-19$ years being obese. Obesity is associated with heart disease, stroke, type 2 diabetes, and some kinds of cancer. Obesity is estimated to cost the U.S. \$147 billion annually, and healthcare costs of obese people are approximately $\$ 1,429$ more than those of individuals with normal weights. Type 2 diabetes is further associated with cardiovascular disorders, and left untreated, can cause necrosis of digits and appendages. Perhaps the most disturbing aspect of this trend is that these disease etiologies are completely preventable.

Often when feeding is the focus, attention is either on the forebrain or the hypothalamus. Yet, we know the VTA is a site of extensive neural convergence, offering unparalleled signal processing for the early stages of motor output and behavioral determination. Since the PBN, the second relay of the taste system, also located in the brainstem, provides some of these inputs, it is reasonable to hypothesize this connection may function to partially control feeding. To test this, I designed the project described in 
this dissertation to investigate the direct neural pathway from the PBN to the VTA and whether it provides such functional connectivity to the taste and reward systems.

First, I want to note that after this preface, I chose to use the collective pronoun "we" as opposed to "I". None of the work done herein was solely completed by me alone, as noted in the acknowledgements. Thus, with the team effort invested in completing the projects presented, I chose to write my dissertation with a style reflective of this fact. However, as these stylistic choices as well as those regarding chapter structure were mine, in the preface, I use I/me/my.

Secondly, I found myself facing the decision of whether to write a dissertation on feeding that happened to be influenced by taste via the reward system or on an interaction between taste and reward that happened to influence feeding. My decision was to write the latter. As such, the following choices about chapter organization were made.

In the first four chapters, I summarize the history and science of taste, reward, and feeding. I begin by defining relevant phenomena and provide examples to ensure a priori clarity about the field's concepts and what I investigated. Further, I introduce the background most salient to my experiments, which is elaborated on in the three literature review chapters that follow (Chapters 2 - 4). I found that providing the history of the science of feeding was most challenging to cover because less had been done to compile its philosophical foundation. Thus, the historical perspectives focused on taste and reward. The second and third chapters expound the neurobiology of taste and reward, respectively.

The fourth chapter narrows down on the research concerning the PBN and VTA. I explain the important anatomical and physiological findings of these areas. I continue by pointing out their connectivity, finishing with my proposal to test their collective involvement in taste reward and feeding. I conclude this chapter by outlining the aims of my experiments.

Naturally, the fifth through eighth chapters detail the methods used in the investigation as well as the results of each. The fifth chapter provides general methodology which was used in multiple parts of the experiments that follow. Each of chapters six to eight provides more specific methodology as well as what was found resulting from investigations into the PBN, VTA, and circuit/nuclei manipulations, respectively.

Chapter nine discusses findings specific to each of the investigations as well as relationships of the findings across investigations. After making some final statements about the limitations of the methodology, I briefly conclude the discussion by predicting the future of this field and philosophizing on this dissertation's findings. 


\section{TABLE OF CONTENTS}

\section{CHAPTER 1. HISTORICAL PERSPECTIVES ON TASTE AND REWARD, AND HOW THEY RELATE TO FEEDING ....................................................................1}

Taste 1

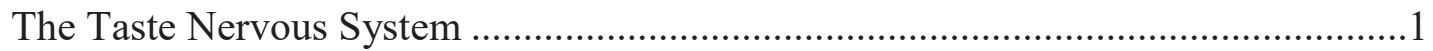

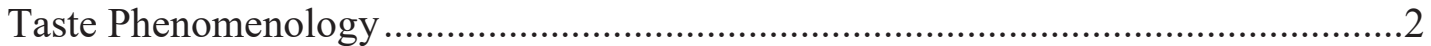

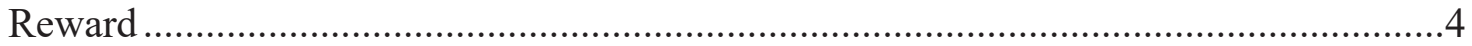

Historical Perspective of Reward.......................................................................4

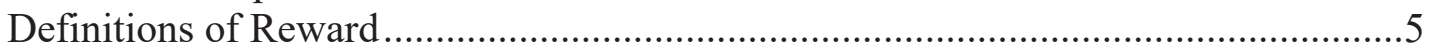

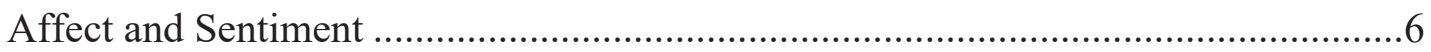

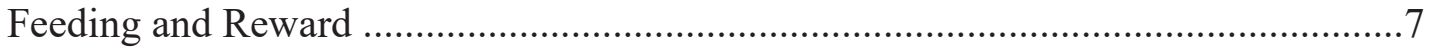

CHAPTER 2. NEUROBIOLOGY OF THE TASTE SYSTEM..................................9

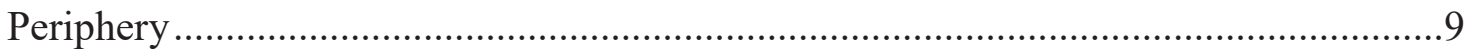

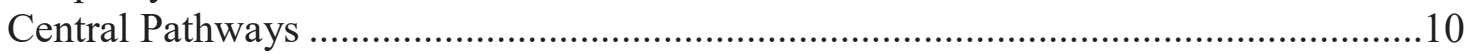

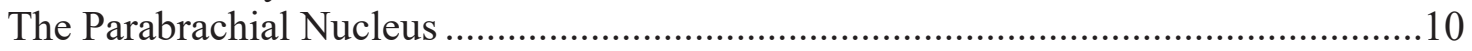

CHAPTER 3. THE NEUROBIOLOGY OF REWARD ................................................14

The Role of the Dopamine System in Reward .........................................................14

Dopamine Neurons Mediate Urge in Response to Rewards .....................................14

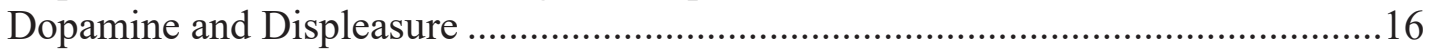

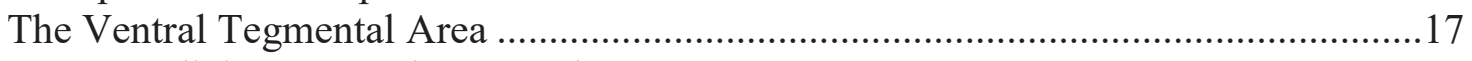

Rostromedial Tegmental Area and Non-DA VTA Neurons .....................................18

The Neurobiology of Affect in Taste and Feeding ................................................. 19

\section{CHAPTER 4. THE TASTE/REWARD INTERFACE OF THE PBN AND VTA ....21}

Direct and Indirect Connectivity between Taste and Reward Areas ............................21

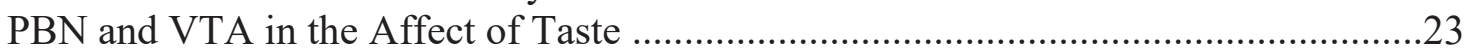

Post-ingestive and Taste Interactions .................................................................24

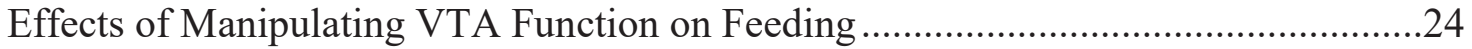

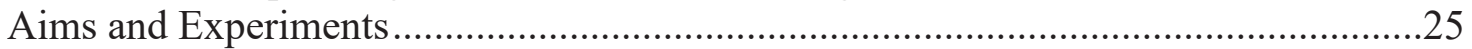

Analyze the PBN Response in Relation to Taste Valence and Characterize its

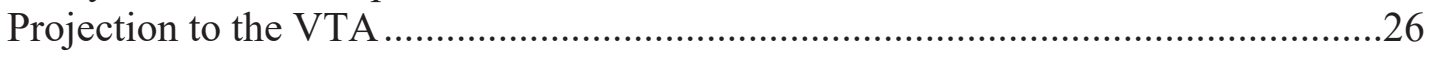

Determine the Location and Morphology of VTA Neurons Responsive to Taste

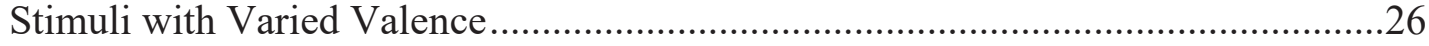

Modulate Feeding by Manipulating the VTA with DREADDs and Drugs ..............28

CHAPTER 5. GENERAL METHODOLOGY ...................................................29

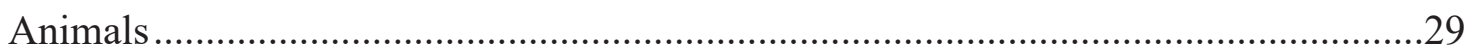

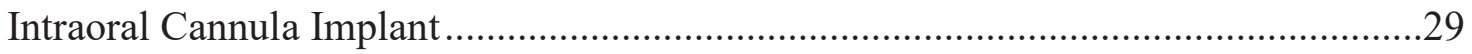

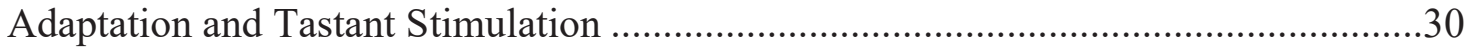

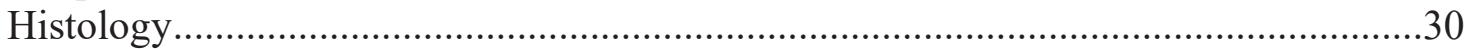

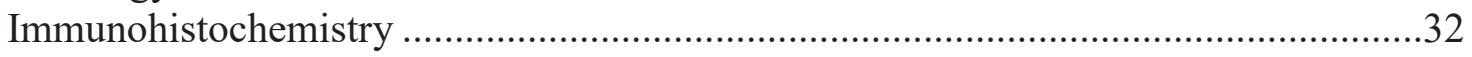


Imaging

\section{CHAPTER 6. TASTE-EVOKED ACTIVITY IN PBN NEURONS INCLUDING} PROJECTIONS TO EITHER THE VTA OR GUSTATORY THALAMUS ...........33

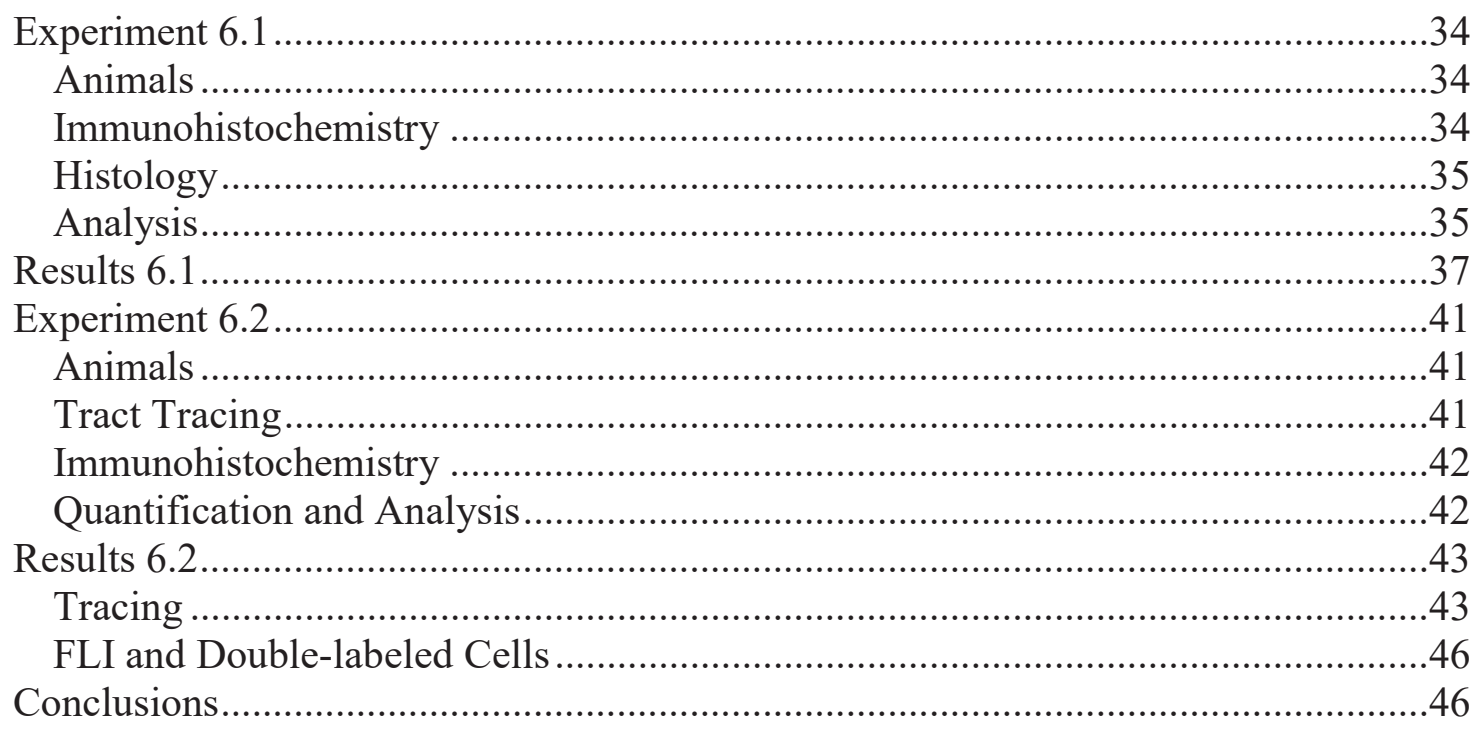

\section{CHAPTER 7. LOCATION AND MORPHOLOGY OF VTA NEURONS} RESPONSIVE TO TASTE STIMULI WITH VARIED VALENCE .........................50

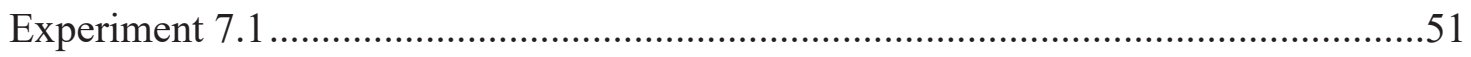

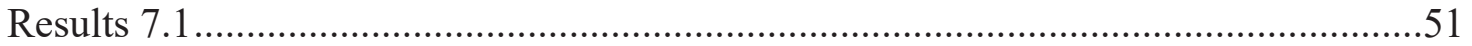

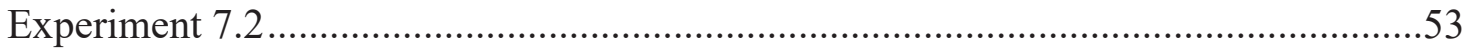

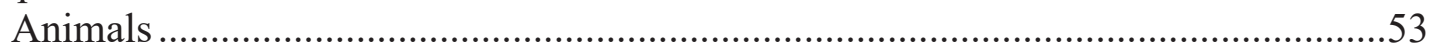

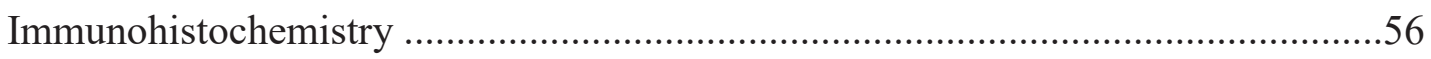

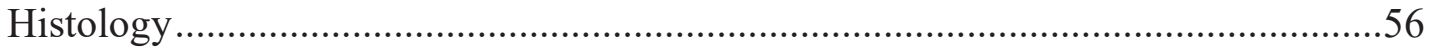

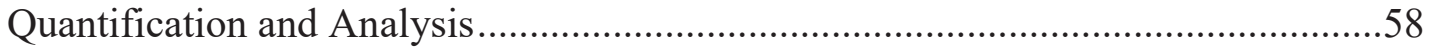

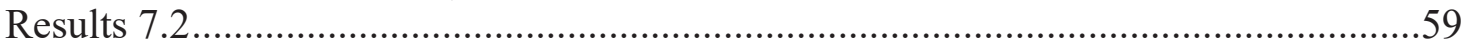

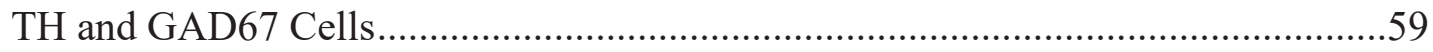

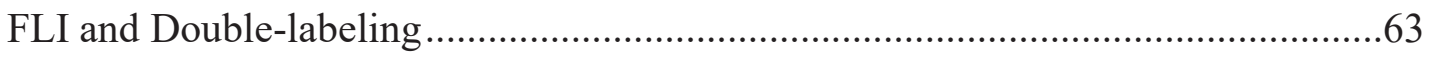

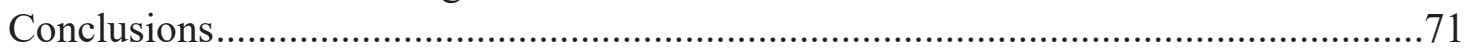

\section{CHAPTER 8. MODULATION OF FEEDING BY MANIPULATING THE VTA ..76}

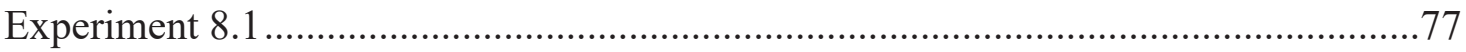

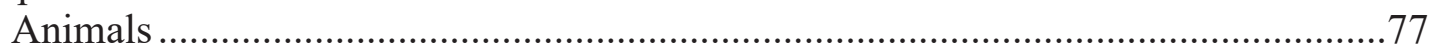

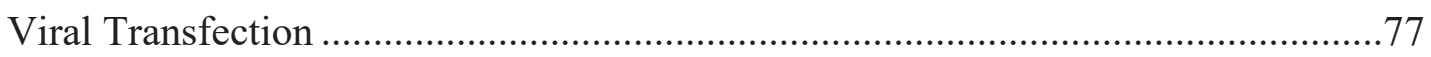

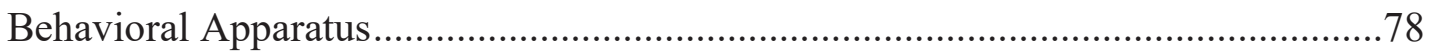

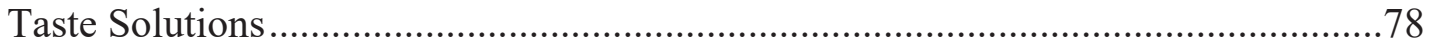

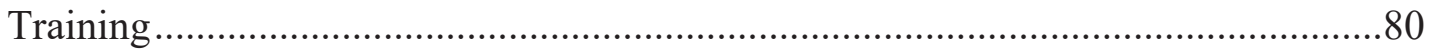

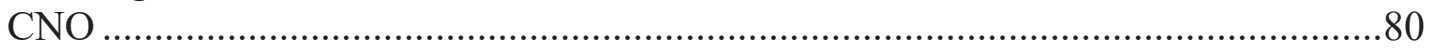

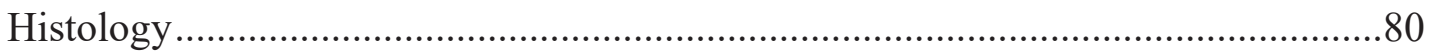

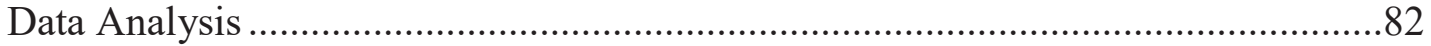

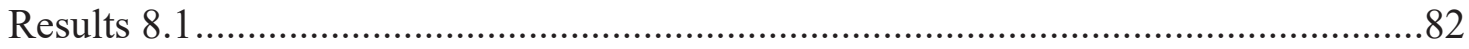

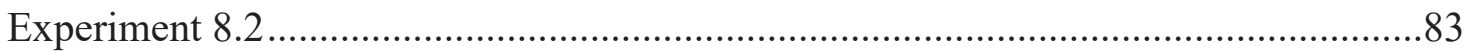


Animals

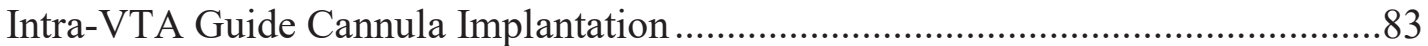

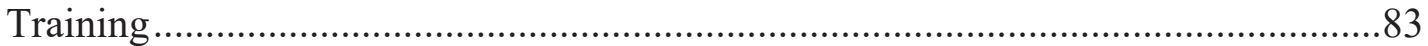

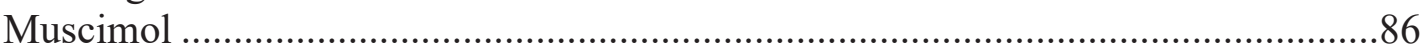

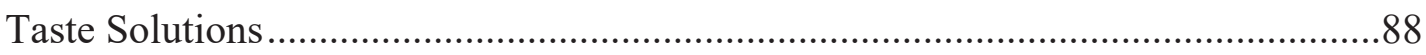

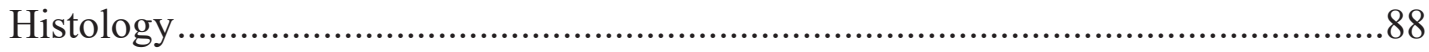

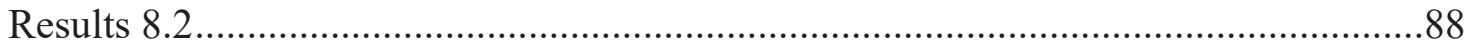

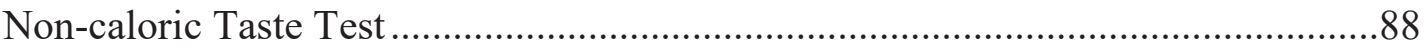

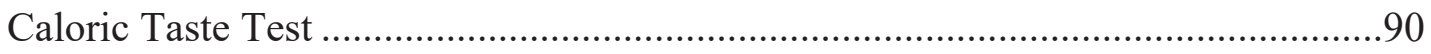

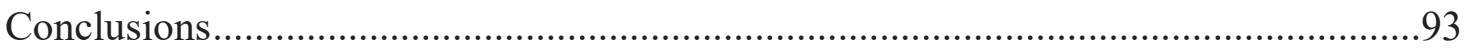

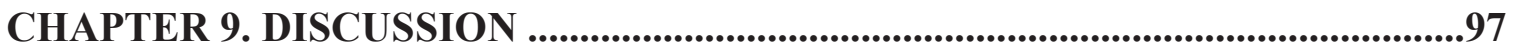

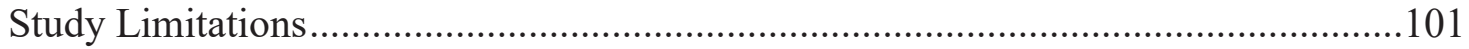

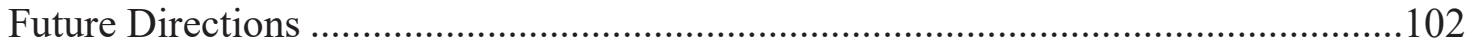

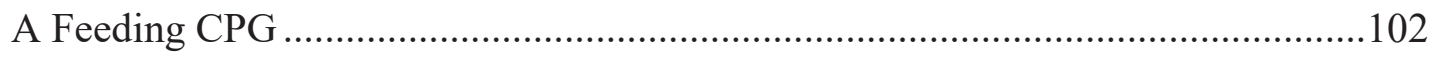

Learning in Feeding and Synaptic Adaptation......................................................103

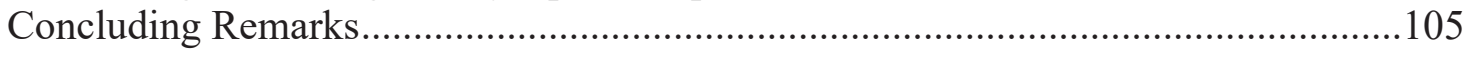

LIST OF REFERENCES ......................................................................................106

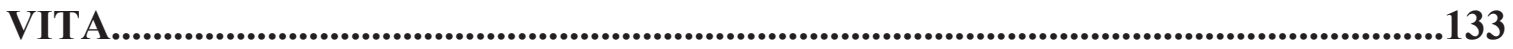




\section{LIST OF TABLES}

Table 1-1. Terms used for taste, reward, and feeding phenomena................................

Table 5-1. Stimuli used in anatomical, physiological, and behavioral studies. .............31

Table 7-1. $\quad$ Results of ANOVAs at VTA AP levels...................................................54

Table 7-2. Results of ANOVAs testing AP level for each strain/stimulus group..........55

Table 7-3. Effects in VTA GAD67 + FLI double-labeled neurons.............................72

Table 7-4. Effects in RMTg double-labeled neurons. ............................................73

Table 8-1. PBN-to-VTA activation behavioral paradigm schedule............................81

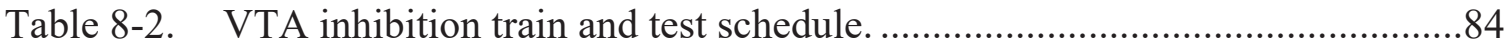

Table 8-3. VTA inhibition water and food partial restriction schedules......................85 


\section{LIST OF FIGURES}

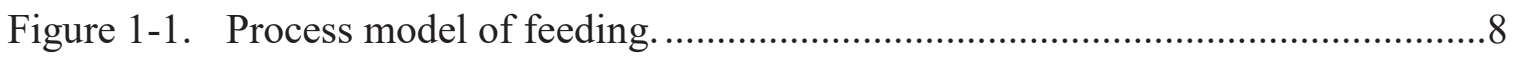

Figure 2-1. Peripheral and central taste pathways.......................................................11

Figure 4-1. Parabrachial nucleus (PBN) fibers and varicosities in midbrain and

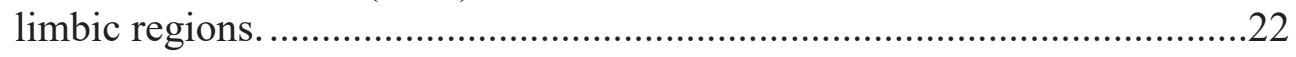

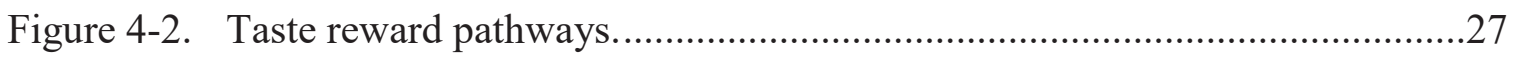

Figure 6-1. Delineation of PBN at 5 rostral-caudal levels..............................................36

Figure 6-2. FLI in PBN measured with DAB immunohistochemistry in B6 and

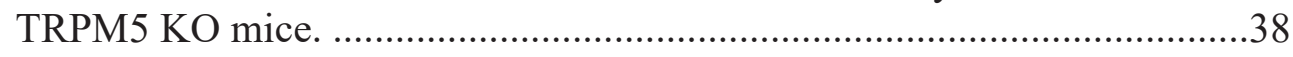

Figure 6-3. FLI in mouse strains and to taste stimuli.....................................................39

Figure 6-4. FLI in C57BL/6J (B6) and trpm5 -/- (KO) mice in PBN. ...........................40

Figure 6-5. VTA and VPMpc injection sites...............................................................

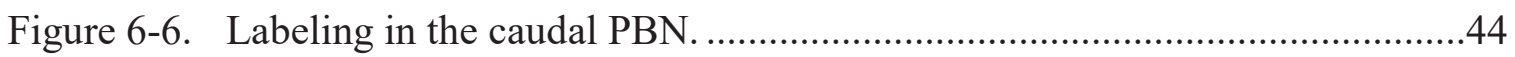

Figure 6-7. Examples of FG, CTb, and FLI labeling in the PBN...................................45

Figure 6-8. Quantification of FG, CTb, and FLI labeling in the PBN by subnucleus.....47

Figure 7-1. FLI in VTA measured with DAB immunohistochemistry in B6 and

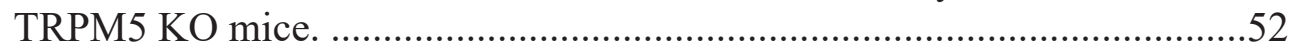

Figure 7-2. FLI in B6 and $\mathrm{KO}$ mice to taste stimuli....................................................54

Figure 7-3. Semi-consecutive representative sections containing VTA subnuclei.........57

Figure 7-4. Examples of taste-elicited c-Fos in TH and GAD67 VTA neurons. .............60

Figure 7-5. TH+ and GAD67+ cells in VTA subnuclei. ..............................................61

Figure 7-6. TH+ and GAD67+ cells in the VTA organized by AP level (mm relative to bregma).

Figure 7-7. TH+ and GAD67+ neurons in the RMTg subnucleus of the VTA...............64

Figure 7-8. FLI ratios in VTA subnuclei to taste stimuli. ..............................................65

Figure 7-9. FLI ratios to taste stimuli in the VTA by anterior-posterior level................66 
Figure $7-10 . \mathrm{TH}+$ FLI counts to taste stimuli in the VTA.....................................6

Figure 7-11. TH + FLI ratios to taste stimuli relative to constituents in VTA subnuclei.

Figure 7-12. TH + FLI ratios to taste stimuli relative to constituents along AP axis of

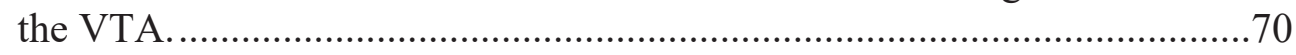

Figure 8-1. Targeted activation of the PBN-to-VTA circuit may affect feeding. ...........79

Figure 8-2. Body weight (BW) fluctuation throughout experiment. ............................87

Figure 8-3. Exemplar of VTA cannula placement indicated by injections. ...................89

Figure 8-4. Test-pretest ratios to non-caloric and caloric taste stimuli. .......................91

Figure 8-5. Cumulative licking in sessions to non-caloric taste stimuli......................92

Figure 8-6. Cumulative licking in sessions to caloric taste stimuli..............................94 


\section{LIST OF ABBREVIATIONS}

\begin{tabular}{|c|c|}
\hline AADC & amino acid decarboxylase \\
\hline AAV & adeno-associated virus \\
\hline AMPA & $\alpha$-amino-3-hydroxy-5-methyl-4-isoxazoleproprionic acid \\
\hline AMY & amygdala \\
\hline AP & anteroposterior \\
\hline ATP & adenosine triphosphate \\
\hline aVTA & anterior VTA \\
\hline B6 & C57BL/6J wild-type mice \\
\hline $\mathrm{BC}$ & brachium conjunctivum \\
\hline BLA & basolateral amygdala \\
\hline $\mathrm{BST} / \mathrm{BNST}$ & bed nucleus of the stria terminalis \\
\hline $\mathrm{BW}$ & body weight \\
\hline $\mathrm{CeA}$ & central amygdala \\
\hline CeAlc & laterocapsular portion of the $\mathrm{CeA}$ \\
\hline CGRP & calcitonin gene-related peptide \\
\hline $\mathrm{cl}$ & central lateral subnucleus of the PBN \\
\hline $\mathrm{CLi}$ & caudal linear nucleus of the raphe of the VTA \\
\hline $\mathrm{CNO}$ & clozapine-N-oxide \\
\hline cNST & caudal, visceral NST \\
\hline $\mathrm{cp}$ & cerebral peduncle \\
\hline $\mathrm{CS}$ & conditioned stimulus \\
\hline CTA & conditioned taste aversion \\
\hline
\end{tabular}




$\begin{array}{ll}\text { CTb } & \text { cholera toxin subunit B } \\ \text { DA } & \text { dopamine } \\ \text { DAergic } & \text { dopaminergic } \\ \text { DAB } & \text { 3, 3'-diaminobenzidine HCl } \\ \text { DAMGO } & \text { [D-Ala2, N-Me-Phe4, Gly5-ol]-enkephalin } \\ \text { DAT } & \text { dopamine transporter } \\ \text { DDS } & \text { dopamine dysregulation syndrome } \\ \text { DL } & \text { double-labeled (cell/count) } \\ \text { dl } & \text { dorsal lateral subnucleus of the PBN } \\ \text { dm } & \text { dorsal medial subnucleus of the PBN } \\ \text { DREADDs } & \text { designer receptors exclusively activated by designer drugs } \\ \text { DV } & \text { dorsoventral } \\ \text { el } & \text { external lateral subnucleus of the PBN } \\ \text { em } & \text { gustatory cortex } \\ \text { ENaCs } & \text { green fluorescent protein } \\ \text { FG } & \text { fornal medial subnucleus of the PBN } \\ \text { FLI } & \text { epithelial sodium channels } \\ \text { fMRI } & \text { Fluoro-Gold } \\ \text { fr } & \text { functional magnetic resonance imaging } \\ \text { GABA } & \text { glutamate decarboxylase } \\ \text { GAD } & \end{array}$




\begin{tabular}{|c|c|}
\hline i.o. & intraoral/intraorally \\
\hline i.g. & intragastric \\
\hline i.p. & intraperitoneal \\
\hline IEG & immediate early gene \\
\hline IF & interfascicular nucleus of the VTA \\
\hline il & internal lateral subnucleus of the PBN \\
\hline IP & interpeduncular area \\
\hline IPN & interpeduncular nucleus \\
\hline KF & Kölliker-Fuse nucleus \\
\hline KO & trpm 5 -/- knockout mice \\
\hline $\mathrm{LC}$ & locus coeruleus \\
\hline L-DOPA & L-3,4-dihydroxyphenylalanine \\
\hline LDTg & laterodorsal tegmental area \\
\hline LH & lateral hypothalamus \\
\hline 1PBP & lateral PBP \\
\hline 1VTA & lateral VTA \\
\hline $\mathrm{m}$ & medial subnucleus of the PBN \\
\hline $\mathrm{MCH}$ & melanin concentrating hormone \\
\hline Me5 & mesencephalic trigeminal nucleus \\
\hline ML & mediolateral \\
\hline $\mathrm{ml}$ & medial lemniscus \\
\hline MM & mammillary nucleus \\
\hline $\mathrm{mPBP}$ & medial PBP \\
\hline
\end{tabular}




\begin{tabular}{|c|c|}
\hline $\mathrm{mPFC}$ & medial PFC \\
\hline $\mathrm{mt}$ & mammillothalamic tract \\
\hline mVTA & medial VTA \\
\hline NAc & nucleus accumbens \\
\hline NAcc & core of the NAc \\
\hline NAcs & shell of the NAc \\
\hline NMDA & N-methyl-D-aspartate \\
\hline NST & nucleus of the solitary tract \\
\hline OFC & orbitofrontal cortex \\
\hline $\mathrm{OX}$ & orexin \\
\hline PAP & peroxidase anti-peroxidase \\
\hline PB & phosphate buffer \\
\hline $\mathrm{PbN} / \mathrm{PBN}$ & parabrachial nucleus \\
\hline PBP & pigmented parabrachial nucleus of the VTA \\
\hline PBS & phosphate buffered saline \\
\hline PBTx & $0.8 \%$ triton-X 100 in $0.1 \mathrm{M} \mathrm{PB}$ \\
\hline PF & parafascicular thalamic nucleus \\
\hline PFC & prefrontal cortex \\
\hline $\mathrm{PH}$ & posterior hypothalamus \\
\hline $\mathrm{PN}$ & paranigral nucleus of the VTA \\
\hline pVTA & posterior VTA \\
\hline $\mathrm{QHCl}$ & quinine hydrochloride \\
\hline RF & reticular formation \\
\hline
\end{tabular}




\begin{tabular}{|c|c|}
\hline RLi & rostral linear nucleus of the raphe of the VTA \\
\hline RMTg & rostromedial tegmental area of the VTA \\
\hline $\mathrm{RN}$ & red nucleus \\
\hline rNST & rostral, gustatory NST \\
\hline RRF & retrorubral field \\
\hline $\operatorname{scp}$ & superior cerebellar peduncle \\
\hline $\mathrm{SN}$ & substantia nigra \\
\hline $\mathrm{SNC} / \mathrm{SNpc}$ & substantia nigra, pars compacta \\
\hline $\mathrm{SNR} / \mathrm{SNpr}$ & substantia nigra, pars reticulata \\
\hline $\operatorname{subN}$ & subnucleus \\
\hline T1R & sweet taste receptor \\
\hline $\mathrm{T} 2 \mathrm{R}$ & bitter taste receptor \\
\hline $\mathrm{TH}$ & tyrosine hydroxylase \\
\hline $\mathrm{THC}$ & $\Delta$-9-tetrahydrocannabinol \\
\hline TRC & taste receptor cell \\
\hline TRPM5 & transient receptor potential cation channel subfamily $\mathrm{M}$ member 5 \\
\hline tVTA & "tail" region of the VTA \\
\hline UCS & unconditioned stimulus \\
\hline VGluT2 & vesicular glutamate transporter 2 \\
\hline $\mathrm{vl}$ & ventral lateral subnucleus of the PBN \\
\hline VMAT2 & vesicular monoamine transporter 2 \\
\hline VP & ventral pallidum \\
\hline PM & venteroposteromedial nucleus of the thalamus \\
\hline
\end{tabular}




$\begin{array}{ll}\text { VPMpc } & \begin{array}{l}\text { parvicellular region of the venteroposteromedial nucleus of the } \\ \text { thalamus }\end{array} \\ \text { vsc } & \text { ventral spinocerebellar tract } \\ \text { VTA } & \text { ventral tegmental area } \\ \text { wa } & \text { gustatory waist of the PBN } \\ \text { ZI } & \text { trigeminal motor nucleus } \\ \text { V } & \text { facial motor nucleus } \\ \text { VII } & \text { hypoglossal motor nucleus } \\ \text { XII } & \text { facial nerve } \\ \text { VIIth } & \text { glossopharyngeal nerve } \\ \text { IXth } & \text { vagus nerve } \\ \text { Xth } & \text { 6-hydroxydopamine } \\ \text { 6-OHDA } & \end{array}$




\section{CHAPTER 1. HISTORICAL PERSPECTIVES ON TASTE AND REWARD, AND HOW THEY RELATE TO FEEDING}

The influence of taste on feeding is almost so obvious it might be overlooked as a matter for scientific inquiry. We eat what tastes good. However, we might also eat less appetizing but nutritious foods (e.g., vegetables) and avoid the tastiest but unhealthy foods (e.g., fast food, ice cream). Despite the former, we may also have a lower limit of what we accept at which point nutrition becomes irrelevant (e.g., "spinach is just too yucky"). Further, we may eat unhealthily because of overwhelming urges. We used the experiments of this dissertation to investigate these complex interactions of taste and feeding.

\section{Taste}

To understand how taste influences feeding, we need to begin by defining the taste sensory system and the mechanisms that use that information to generate action. In this case, the taste system forms the interface between our environment and the affective components that later determine decisions about feeding.

\section{The Taste Nervous System}

The Greek philosopher, Alcmaeon (5th century B.C.), was one of the earliest scholars to suggest a mechanism for how the tongue sensed taste ${ }^{1}$. He posited that particles entered pores in the tongue to elicit taste in the "sensorium". Incidentally, he also believed the sensorium, and therefore, the perception of taste, was located in the brain. As one of the chemical senses, we now know molecules do enter pores in the tongue where they can then bind receptors located in the taste buds of papillae ${ }^{2,3}$. The father of anatomy, Galen (2nd century A.D.), hypothesized the necessity that the molecules be solubilized, indicating specifically that the tongue must be moist for it to sense properly ${ }^{1}$. He also correctly identified the glossopharyngeal nerve as one which innervated the tongue and carried taste information centrally. The beginning of the alimentary canal contains taste buds, the most peripheral sense organs of this system, which are innervated by cranial nerves, beginning the communication of taste information centrally ${ }^{4}$. Albrecht von Haller $(1708-1777)$ was the first to describe papillae, the epithelial tongue structures which "house" taste buds ${ }^{1}$. Taste buds, composed of taste receptor cells (TRCs) and basal cells ${ }^{2,3}$, were discovered later by Gustav Schwalbe $(1844-1916)$ and Otto Christian Lovén $\left(1835\right.$ - 1904) ${ }^{1}$. Carlo Francesco Bellingeri (1789 - 1848) discovered that the chorda tympani branch of the facial nerve conducted taste from the anterior tongue (i.e. from fungiform taste buds) more than a millennium after Galen's identification of the function of the glossopharyngeal ${ }^{1}$. Discoveries of the taste system's central pathways began in the 19th century with lesion studies in animals ${ }^{1}$. These were followed by human neuropsychology cases and advancements in neural tracing. 


\section{Taste Phenomenology}

The sensory modality of taste is also known as "gustation". Two aspects of taste sensation that can be discerned psychophysically are the basic taste categories (i.e., qualities) and taste valence (i.e., the perceptual identification of a taste stimulus' hedonic affect) ${ }^{5}$. Aristotle (4th century B.C.) correctly identified bitter, salty, sour, and sweet as basic tastes, although he also included astringent, harsh, and pungent in his list ${ }^{1,6,7}$. A fifth taste known as umami (Japanese word meaning "good taste"; refers to a savory taste sensation) elicited primarily by certain L-type amino acids was first described by Ikeda in $1909^{8}$. Having entered the common parlance, umami taste is widely known and accepted as a distinct primary taste, perhaps especially following the discovery of specific taste receptors for its prototypical stimuli. Whether there is a distinct quality for fat and other tastes is still debated. In conjunction with taste, other sensations contribute to perceptions of food. Olfaction (i.e., smell) combines with taste to produce flavor, a complex perception to which somatosensation (texture) and pain (spicy) may also contribute. Although all of these sensations may occur to one substance in the oral cavity, taste is not the same as flavor. Interestingly, recent findings have even shown vision and hearing may interact with taste ${ }^{9}$, but no consensus establishes whether this interaction is impacting flavor or a separate and more complex perception.

Taste is also associated with other psychological phenomena such as affect, reward, and motivation. Hedonic impact indicates how palatable or aversive a taste is and is measured using taste reactivity ${ }^{10}$. In animals with less developed brains, such as rodents and young primates, stereotypical facial expressions and behaviors can be used to quantify hedonic impact in taste reactivity tests. Positive hedonic impact (i.e., evidence of a pleasurable taste) is a necessary component of the development of incentive salience related to taste (see below), an attribute of taste stimuli (and associated non-taste stimuli) which causes or primes approach and/or ingestion (i.e., makes the taste appetitive). While it is known that negative hedonic impact (i.e., evidence of displeasure) of taste stimuli is associated with avoidance and/or egestion, no counterpart to incentive salience which would cause or prime these behaviors (i.e., make them repulsive) has been identified. The positive and negative extremes of hedonic impact might be taken to imply that the underlying neurobiology can be sufficiently explained by associating it with one dimension of affect, however others link the neurobiological systems with two or more orthogonal dimensions. Further attention to the relationship of neurobiology with affective dimensions will be given below in the section on reward.

Care was taken here to avoid conflating one of the sensory aspects of taste, valence, with the affective aspect, hedonics (see Table 1-1). (While this hypothetical distinction may not ubiquitously align with the terminology used by other researchers, attention was paid to ensure these terms were used consistently within this dissertation.) In addition to quality, humans and animals can innately sense valence of taste stimuli, the two extremes of which are like and dislike. Aristotle may have been the first to identify taste valence, as he stated sweet and bitter were the two extremes between which the other qualities lay. However, this conceptualization conflates quality and valence. Indeed, 
Table 1-1. Terms used for taste, reward, and feeding phenomena.

\begin{tabular}{lcc}
\hline & \multicolumn{2}{c}{ Terms } \\
\cline { 2 - 3 } Phenomenon & Like & Dislike \\
\hline Perceptual Identification of Taste Stimulus's & & \\
Hedonic Affect & Pleasure & Displeasure \\
Salience & Incentive & Disincentive \\
Taste Hedonic = HedonicAffect*Taste & Palatable & Aversive \\
Taste Salience = TasteHedonic*Urge & Appetitive & Repulsive \\
Taste Reactivity (i.e., Facial/Behavioral Expression) & Positive & Negative \\
Locomotion & Approach & Avoidance \\
Feeding Behavior & Consume, Ingest & Expel, Egest \\
\hline
\end{tabular}


one is challenged not to conflate these, as the sensory and affective phenomena are typically experienced together. Teasing them apart has only been possible in more recent neurobiological experiments capable of specifically inhibiting each of their underlying mechanisms ${ }^{5,10}$ (see Marks, $2011^{11}$ ). In this dissertation, taste liking is the sensory experience associated with substances that are avidly ingested and elicit pleasure, and disliking is associated with substances that are avoided or egested and elicit displeasure. Both valence and hedonic aspects relate taste to feeding as will be further explained below.

\section{Reward}

Once the taste system has transduced stimuli from our environment into neurochemical signals, our brains can use the information to generate affect. Affect is related to reward and emotion, but all three rely on different neurobiology. Nonetheless, sensations like taste can contribute to reward and affect, and understanding the affect of taste helps explain how this sense contributes to the modulation of feeding.

\section{Historical Perspective of Reward}

The first documented ideas about reward date back to four millennia ago when the poem "Song of the Harper" was inscribed on the tomb of the Egyptian King Intef ${ }^{11}$. Its verses lament the finality of death and urge the reader to enjoy the pleasures of life in the present. This philosophy came to be known as hedonism. It would later be incorporated in the utilitarian theory of Jeremy Bentham $(1748-1842)$, an influence on scientific thought in the 19th century, including on motivation and reward. Aristotle wrote presciently about motivation and pleasure in De Anima and De Sensu ${ }^{11}$. In trying to describe how sensory experience relates to knowledge, he asked questions about how to measure and qualify pleasure. He wrote about the contingency that can occur with conditional pleasures and biological states as when some deprivation states can elicit hunger for foods that alleviate that deprivation specifically. This phenomenon would later be described by Michel Cabanac as alliesthesia ${ }^{12}$. Aristotle also predicted that pleasure occurs through different processes than motivation, a hypothesis later confirmed by Kent C. Berridge ${ }^{10}$.

The delineation of sensation and reward increased as thinking came to specify how the nervous system worked in detail ${ }^{11}$. Johannes Müller $(1801-1858)$ explained in his doctrine of "specific energies of the nerve" that sensations were due to activity in the tracts in the brain rather than dependent on the associated modality's stimuli. Müller's student, Herman von Helmholtz, also implicated pleasure's independence from stimuli, as Müller had of all qualities of sensation. Oswald Külpe further differentiated pleasure, a feeling, would be parallel to sensation instead of subsumed by it.

By the 20th century, the rich understanding of reward coupled with advancing techniques allowed science to begin empirically validating these hypotheses about 
sensation and pleasure ${ }^{11,13}$. Leonard Troland introduced beneceptors, nociceptors, and neutroceptors, each with a corresponding relationship to its associated stimuli's benefit or harm. He attempted to explain motivation based on how stimuli activated these receptors. James Olds and Peter Milner confirmed the ability to drive behavior with electrical brain stimulation by training rats to press a bar for administration. Ivan Pavlov had already shown classical conditioning could drive dogs to salivate at the sound of a bell. But Olds and Milner thought they were providing a neurological explanation for Edward L. Thorndike's Law of Effect, which originally suggested that responses which are satisfying (i.e., rewarding) are repeated, a fundamental principle that explains how operant conditioning occurs. More importantly, applying Thorndike's principle indicates feeding-related behaviors which are rewarding - whether in a taste or visceral capacitywill be repeated.

\section{Definitions of Reward}

According to Berridge, reward includes distinguishable parts of pleasure, learning, and incentive salience ${ }^{10,14}$. Rewards are stimuli which themselves elicit pleasure or are associated with stimuli that elicit pleasure. Associated rewards attain their status through learning. The brain transmitter dopamine (DA) is commonly associated with the concept of reward in popular culture, which in turn is often erroneously equated with pleasure. For example, consider a current article about "the science of love" on an educational neuroscience website (www.youramazingbrain.org ${ }^{15}$ ):

“...newly 'love struck' couples [had] their brains examined and discovered they have high levels of the neurotransmitter dopamine. This chemical stimulates 'desire and reward' by triggering an intense rush of pleasure".

In fact, landmark studies by Wolfram Schultz showed that activity in midbrain DA neurons (and their resulting DA release in forebrain targets) encode reward prediction error, a result of the learning process which associates inherently pleasurable rewards with associated stimuli ${ }^{16}$. However, DA is not itself responsible for mediating pleasure (see Chapter 3, especially the section titled The Role of the Dopamine System in Reward) or the learning process ${ }^{17}$. Instead, DA release causes incentive salience, which is an attribute of a percept of a stimulus that causes approach and/or consumption of that stimulus, or primes the approach and/or consumption of another stimulus. Although incentive salience involves the motivational value of a percept of a stimulus, it lacks the targeting aspects necessary to guide the approach or consumption directly to that specific stimulus. In other words, incentive salience is a general urge to act that happens to occur when an organism is exposed to a reward.

An additional distinction is worth making between pleasure and meaning. The ancient Greeks referred to two forms of happiness as hedonia (pleasure or euphoria) and eudaimonia (meaningful, contentment, or joy) ${ }^{18,19}$. While it is generally accepted that rewards are stimuli that elicit pleasure or are associated with stimuli that elicit pleasure, meaningful experiences do not fit this definition and may not work through the same 
neurological mechanism. Eating and sex are inherently pleasurable and lead to the development of incentive salience through the DA system's mechanism described above. However, it is not clear how meaningful experiences like those described by Viktor Frankl would fit into Berridge's theory of DA and reward. Frankl describes creating works of art and science, nurturing close relationships, and the challenging act of choosing to mentally overcome suffering as examples of meaningful experiences ${ }^{20}$. Nascent evidence from the treatment of depression with serotonin modulators and the recreational and medicinal use of psychedelics and entheogens suggests the serotonin system underlies the conscious experience of meaning ${ }^{21-30}$. Further work may be needed to verify that the experience of meaning is due to a separate neurobiological mechanism.

For this dissertation, feeding and its associated affect are generated in such a way that Berridge's definition of reward is adopted. Some foods happen to elicit pleasure. We learn to eat the foods we find pleasurable. The incentive salience of associated cues causes or primes us to approach and consume them. However, avoiding and egesting are not explained by incentive salience; thus, the addition of an orthogonal dimension of affect, displeasure, is necessary. This dissertation extends the general urge to act beyond Berridge's conceptualization to include the driving of behaviors which occurs upon exposure to aversive stimuli. The interplay of neurobiological mechanisms associated with urge and displeasure is elaborated in Chapter 3.

\section{Affect and Sentiment}

To extrapolate on these definitions of reward, a delineation of affect and sentiment based on Baruch Spinoza's Ethics Part III is provided here ${ }^{31}$. Sentiments are experiences of emotions, and emotions, themselves, are physiological responses to events (e.g., the release of epinephrine at the sight of a 12 point buck). While emotion occurs subconsciously, sentiment is a conscious experience resulting from the emotion (e.g., feeling surprised after epinephrine binds receptors in the hypothalamus). Affect is one of three aspects of sentiments and can be isolated from the other two-sensation and cognition-according to how they are perceived temporally in relation to their associated event (Papanicolaou, personal communication ${ }^{32}$ ). While sensation and cognition are identifiable immediately, affect can only be recognized in retrospect (i.e., the sight of the buck caused urge). Due to the phenomena associated with affect, one may choose to prolong, repeat, prevent, or end an experience (e.g., urge which leads to crouching down, stalking, and spearing the buck; pleasure while feeding on venison). An empirical validation using neurobiological studies to delineate affect from sentiment is possible but outside the scope of this introduction.

Affect's relationship with reward is straightforward: Pleasure is one dimension of affect and a recognized component of reward. Additional dimensions of affect are displeasure, urge, and meaning. Earlier, urge was suggested as halfway to being synonymous with Berridge's incentive salience (by excluding the negative component that relates urge to displeasure), and meaning was differentiated from pleasure. The neurobiology of pleasure as well as the DA system and urge will be treated in more detail 
in Chapter 3, and meaning was already indicated as plausibly being mediated by the serotonin system. Displeasure seems it could be of the same mechanism as pleasure but working in the opposite direction. However, phenomenological evidence suggests it is a separate dimension associated with a different neurobiological mechanism. For example, one may simultaneously experience pleasure in eating their favorite food and displeasure in knowing they will soon have consumed the entire meal. Thus, displeasure may be mediated via neural mechanisms other than those that subserve pleasure.

\section{Feeding and Reward}

Feeding, or the consumption of nutritious substances (e.g., calories for energy), is fundamental to life. We may eat to satisfy metabolic needs (homeostasis), to experience pleasure (palatability, 'liking'), and/or due to an urge/incentive/motivation (appetitive, 'wanting') (Figure 1-1). Feeding (i.e., eating) primarily involves the two stages, hunger and satiety, and is influenced by the reward system described above. Depending on their affect, tastes are a powerful factor that may increase or decrease feeding.

Our survival as a species was in part dependent on our ability to feed in effective and safe ways. Evolution favored those of us who developed mechanisms that aided these processes. Some tastes are inherently aversive (e.g., bitter), and the urge and displeasure we experience from these prime or cause us to avoid consuming substances with these tastes. For example, the majority of toxins found in nature have bitter, aversive tastes. Some tastes are inherently appetitive (e.g., sweet), and the urge and pleasure we experience from these prime or cause us to approach and consume substances with these tastes. The systems at work promote survival by performing the heuristic function of indicating which foods are toxic and which are nutritious.

The subjects of taste and reward begin to overlap when one considers the psychology of feeding, and an additional subject arises in learning associations about foods, tastes, and post-ingestive effects. As food enters the alimentary canal, soluble molecules begin to bind taste receptors and tastes are sensed. If the food is palatable, pleasure may be experienced. If the food is appetitive, an urge to continue consuming may be perceived. In contrast, an aversive substance may elicit displeasure and provoke rejection and lead to spitting or some behavior to allow passive drainage. "[H]umans and rats alike tend to eat less of food that is unpalatable, and to seek and eat more when available foods are more palatable" ${ }^{33-38}$. However, if an animal is sickened and caused gastrointestinal malaise after consuming even the most palatable substance, if that substance is novel, the animal will develop an aversion to that substance and others that taste similar (i.e., a conditioned taste aversion [CTA]). Although learning processes are likely mediated at least in part through these systems, the full extent of their involvement is outside the scope of this dissertation. However, effects mediated through post-ingestive interaction is addressed briefly. 


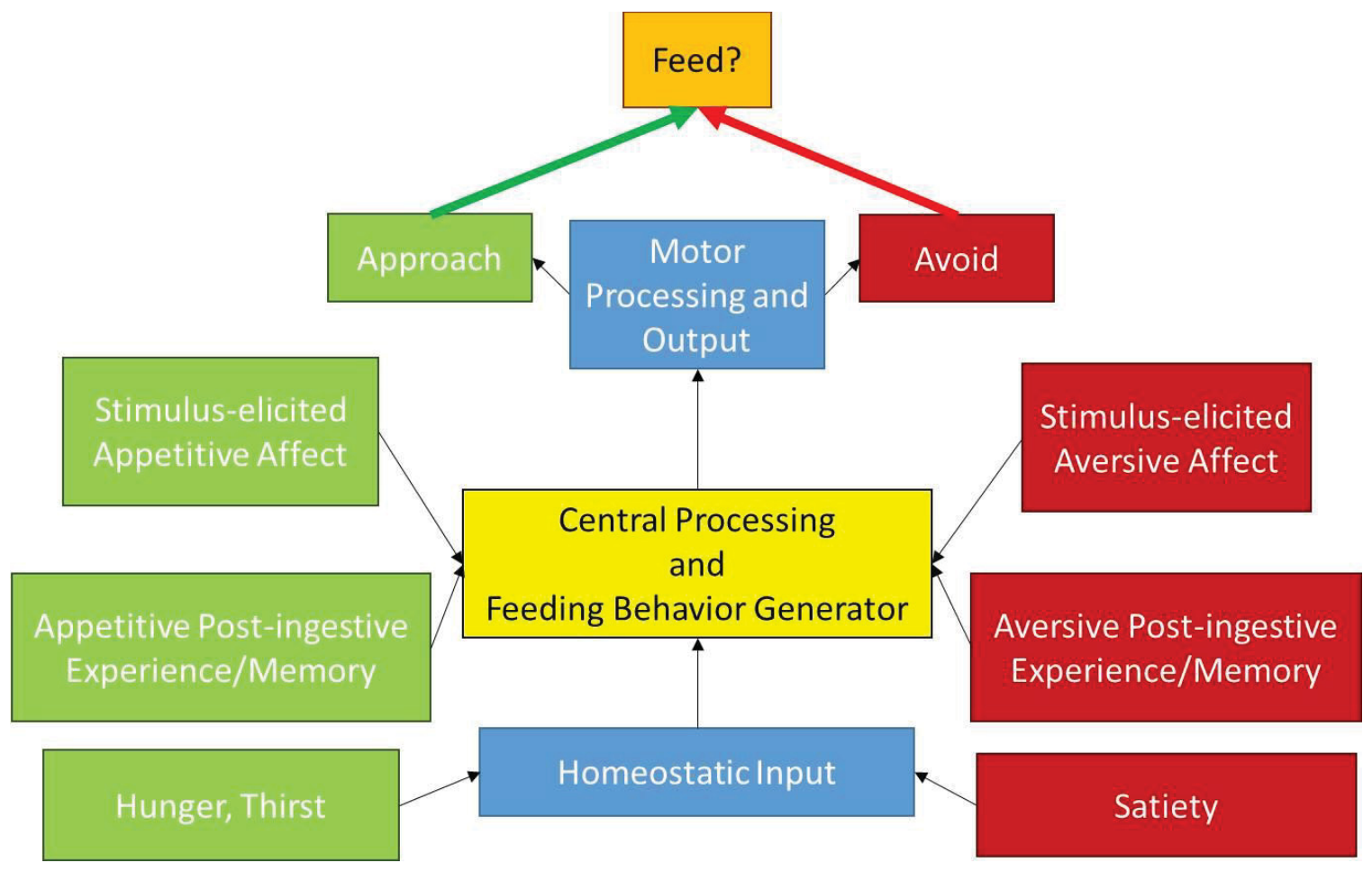

Figure 1-1. Process model of feeding.

Factors of congruent and opposing valence are integrated in decisions to approach, feed, or avoid potentially edible substances. Appetitive inputs and outputs (left side) increase feeding. Aversive inputs and outputs (right side) decrease feeding. Integration occurs in systems represented in the middle, which ultimately lead to decisions and behaviors. 


\section{CHAPTER 2. NEUROBIOLOGY OF THE TASTE SYSTEM}

\section{Periphery}

The sensory process of taste begins when substances are consumed, and dissolved stimuli interact with the apical surface of taste cells in the oral cavity. Taste cells are modified neuroepithelial cells grouped together in discrete units called taste buds ${ }^{2}$. Taste buds are found within papillae on the tongue as well as in other places in the oral cavity, on the soft palate, epiglottis, and in rodents within the nasoincisor duct, located anteriorly on the hard palate. In humans and other mammals, the three different types of papillae that contain taste buds are called fungiform (on the anterior tongue), circumvallate or vallate (posterior tongue), and foliate (sides of the tongue). Within the taste bud, there are anatomically- and molecularly-defined subsets of TRCs, as well as basal cells, which serve as TRC precursors; TRCs have a lifespan of approximately 7-10 days, and are replaced from the basal cell population. Traditionally, three types of TRCs were classified based on morphology, called Type I, Type II, and Type III cells. However, another way to categorize TRCs is according to which of the basic tastes it responds, which in turn is largely dependent on which taste receptors or mechanisms it expresses. Recent work suggests that many TRCs respond selectively to stimuli of a particular class, as well as a link between function and morphology: Type I cells respond to salts, subsets of Type II cells respond to sweet, umami, or bitter stimuli, and Type III cells respond to $\operatorname{acids}^{2}$.

Transduction mechanisms for taste include both G-protein-coupled receptors, as well as ion channels expressed on the apical surface of TRCs ${ }^{2,3}$. Sweet and umami stimuli bind to receptors in the T1R family, whereas bitter stimuli activate T2R receptors. $\mathrm{NaCl}$, a potent taste stimulus for mammals, is transduced when sodium ions flow down a concentration gradient into the TRC through passive epithelial sodium channels (ENaCs). Surprisingly, the mechanism for taste detection of acids is less established, but thought to involve apical proton currents. Depending on transduction mechanism, activation of these receptors or channels leads to activation of a signal cascade, or direct effects on the TRC's membrane voltage or conductance. Depolarization of the TRC, and/or calcium entry through basolateral cation channels, leads to transmitter release. An essential transmitter between TRCs and sensory nerve fibers is adenosine triphosphate (ATP), although some taste cells express and release other transmitters, such as $\mathrm{\gamma}$-aminobutyric acid (GABA), serotonin, and norepinephrine. It is unclear to what degree these other transmitters contribute to TRC-nerve communication, as there is also evidence for neuromodulation among the TRCs themselves.

Axons of cranial nerves innervating the TRCs carry taste information centrally

and include the facial (VIIth), glossopharyngeal (IXth), and vagus (Xth) nerves ${ }^{39-44}$. The chorda tympani and greater petrosal branches of VIIth innervate taste buds on the anterior tongue (fungiform) and palate, respectively. The IXth innervates circumvallate and foliate taste buds on the posterior tongue. A small amount of taste buds are also found in the oropharynx on the aryepiglottic folds; these are innervated by the superior laryngeal 
branch of $\mathrm{X}^{4}$. Taste sensory neurons composing these cranial nerves are pseudo unipolar cells; their somas are located in the geniculate (VIIth), petrosal (IXth), and nodose ganglia (Xth).

\section{Central Pathways}

Peripheral taste neurons terminate centrally in the brainstem, in the rostral-most portion of the nucleus of the solitary tract (NST) ${ }^{39-47}$. These terminations occur in a rough rostral-caudal order, with VIIth nerve axons terminating in the most rostral part of the NST, and Xth nerve taste axons found most caudally. However, the terminal fields of different nerves overlap to some extent. At even more caudal levels of the NTS, vagal input is visceral. Taste-responsive neurons in the NST are glutamatergic, and in rodents, a majority of these project to and terminate in the parabrachial nucleus (PBN) in the pons 43,48-56; however, central taste pathways in humans and other primates exclude the PBN 57,58 .

From the PBN, two major pathways have been traditionally described, termed thalamocortical and ventral (these pathways originate from the NST in humans). As the name implies, PBN neurons projecting to the forebrain as part of the thalamocortical pathway target the thalamic taste area, which is located in the parvicellular region of the venteroposteromedial nucleus of the thalamus (VPMpc). Taste-responsive thalamic neurons in turn project to the primary gustatory cortex (GC), which comprises part of the insular cortex. On the other hand, the ventral pathway includes known projections from the PBN to closely situated limbic forebrain structures such as the central amygdala (CeA), bed nucleus of the stria terminalis (BNST), and lateral hypothalamus (LH) ${ }^{43,59-64}$. This dual-pathway classification is certainly an oversimplified concept, as individual neurons often collateralize to both pathways, and most of the gustatory target brain regions are interconnected. Still, conceptually its value has been in separating presumed functions, with the thalamocortical pathway thought to handle taste quality discrimination and conscious sensory perception, while the projections in the ventral pathway are implicated in hedonics, feeding behavior, association learning and reward. Iron-clad evidence for this segregation of function is lacking, however. A schematic of both the peripheral and central taste pathways is shown in Figure 2-1.

\section{The Parabrachial Nucleus}

The PBN is comprised of cytoarchitecturally-defined groups of neurons surrounding the superior cerebellar peduncle (scp) in the pons, some of which may be considered taste neurons. The PBN functions as an important autonomic and multimodal sensory center in rodents - besides for taste processing, the PBN is a key structure for visceral processing, especially satiety. It also contributes to the processing of somatosensory and pain information, as well as respiratory control ${ }^{65}$. Taste neurons tend to be found at more caudal levels of the PBN ${ }^{49,50}$, and may be multimodal, with some 


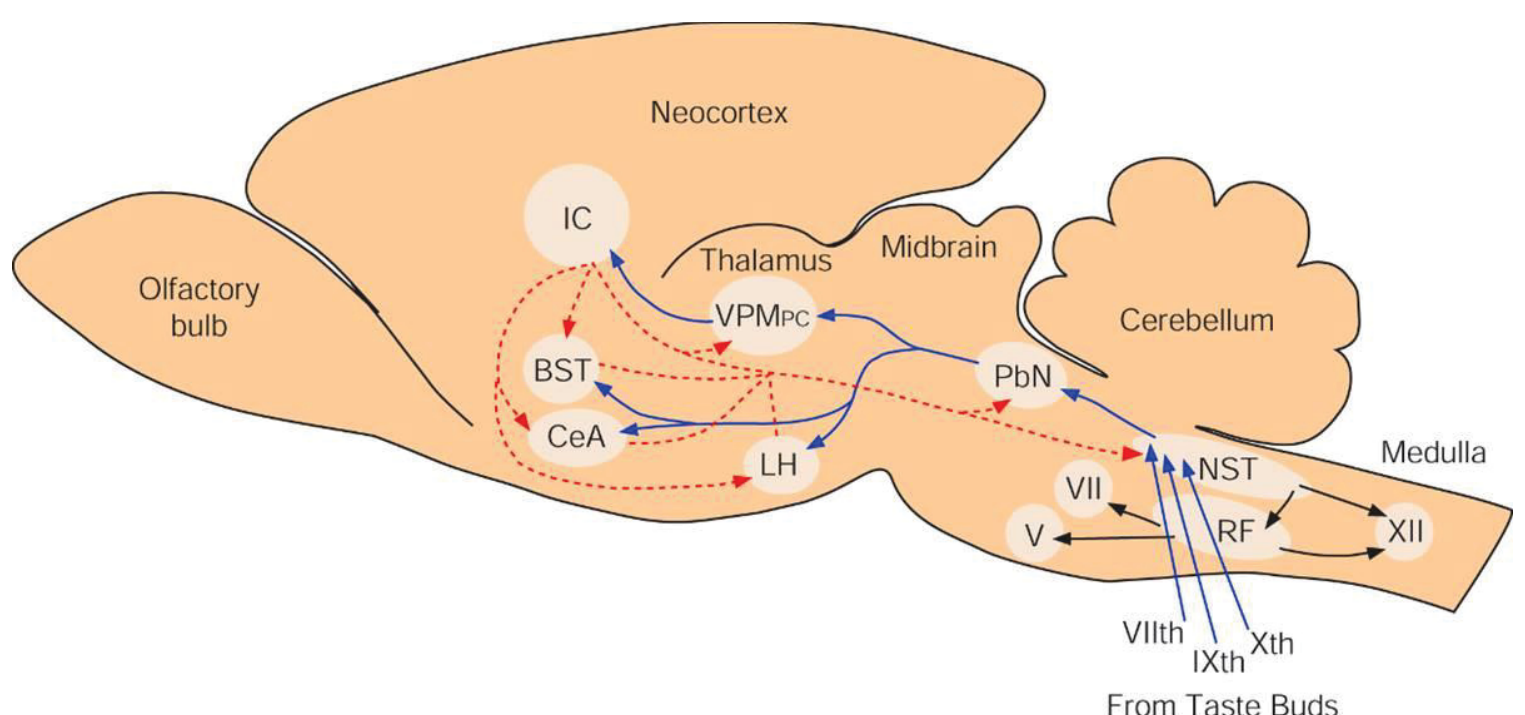

Figure 2-1. Peripheral and central taste pathways.

Taste information enters the central nervous system from facial (VIIth), glossopharyngeal (IXth), and vagus (Xth) cranial nerves. The first relay is the nucleus of the solitary tract (NST) which passes the signal on to the parabrachial nucleus $(\mathrm{PbN})$. From the $\mathrm{PbN}$, the pathways diverge, with one projecting to the parvicellular portion of the venteroposteromedial nucleus of the thalamus (VPMpc) which then innervates the taste portion of the insular cortex (IC). The other pathway projects to ventral forebrain areas, including the lateral hypothalamus ( $\mathrm{LH})$, central amygdala $(\mathrm{CeA})$, and bed nucleus of the stria terminalis (BST). Descending input from all of the nuclei which the $\mathrm{PbN}$ innervates and the IC project back to each of the nuclei lower than it. Ascending pathways are drawn with solid, blue arrows; descending, with dotted, red arrows. Black lines are local circuit projections from NST to medullary nuclei: RF, reticular formation; V, VII, and XII, trigeminal, facial, and hypoglossal motor nuclei.

Reprinted from Encyclopedia of Neuroscience, Smith DV, Boughter JD, Taste: Vertebrate Central Pathways, p. 876, 2009, with permission from Elsevier ${ }^{4}$. 
also responding to tactile or temperature stimuli, as well as others responding to visceral manipulation. Therefore, the PBN appears to be an area of sensory integration.

Gustatory neurons cluster caudally in the PBN in subnuclei found on either side of the white matter tracts of the scp (also called brachium conjunctivum or BC). At caudal levels the BC typically narrows in its center, and PBN neurons are found here crossing between the axon bundles, hence the nickname "waist area", or "gustatory waist" (wa). Electrophysiology studies in the mouse show that taste-responsive cells are found enriched here in several PBN subnuclei, including especially the medial (m) and ventral lateral (vl) subnuclei; they are also found within the BC. Although taste neurons in the PBN can be identified/classified as firing "best" (most robustly) to a particular quality of taste stimulus, their tendency is to respond to multiple taste stimuli, and hence they range in their breadth of tuning ${ }^{45}$. In a recent study in the mouse, taste cells were found that responded best to either sweet, salty, acid or bitter stimuli: Percentages of sucrose-, $\mathrm{NaCl}$-, citric acid-, and quinine hydrochloride $(\mathrm{QHCl})$-best neurons were $42.7 \%, 33.1 \%, 13.5 \%$, and $10.7 \%$, respectively. Overall, studies in rodents show that taste responsiveness in the $\mathrm{PBN}$ is roughly somatotopic, with a greater amount of sucrose-best neurons found medially and within the $\mathrm{BC}$, and a greater amount of $\mathrm{NaCl}$, acid- and QHCl-best neurons found laterally. This pattern may reflect the differential distribution of neurons receiving input (via the NTS) from nerves innervating either the anterior or posterior oral cavity.

In addition to physiological approaches, many studies of taste function in the PBN have utilized expression of the immediate early gene (IEG) $c F$ os to examine neuronal activity (i.e., a proxy for neuronal activation). Early studies in rats showed that intraoral (i.o.) stimulation with basic taste compounds resulted in Fos expression in the PBN that was strongly elevated relative to non-stimulation, and moreover that individual tastes elicited somewhat unique (though partially overlapping) patterns of Fos expression across subnuclei (e.g., Yamamoto et al., $1994^{66}$ ). Furthermore, taste stimulation causes Fos expression in a wider range of PBN subnuclei than predicted by physiology. In addition to the $\mathrm{m}, \mathrm{vl}$, and $\mathrm{BC}$, robust Fos expression is elicited by tastes in the dorsal medial $(\mathrm{dm})$, dorsal lateral (dl), central lateral (cl), and external lateral (el) subnuclei. One likely explanation for this greater pattern of activity in the Fos studies is that while taste stimulation can be restricted to the oral cavity in anesthetized animals during in vivo electrophysiology, i.o. or free-licking stimulation in the awake animals also results in ingestion (depending on stimulus) and this likely provokes Fos expression in neurons that respond to visceral stimulation ${ }^{67}$. Indeed, there is a strong visceral representation in the PBN, especially at more rostral levels, and especially in the el subnucleus ${ }^{68}$. Studies in the mouse showed that two taste stimuli, sucrose and $\mathrm{QHCl}$, resulted in disparate patterns of Fos expression across the PBN (although there is still overlap), including a strong $\mathrm{QHCl}$ signal in the $\mathrm{dm}$, and a strong sucrose signal in the $\mathrm{dl}$.

Understanding taste-related inputs and outputs of the PBN is another method to further analyze how this area encodes, stores, and recalls gustatory information. Tracing studies show that PBN neurons located in most subnuclei project to the thalamus, especially the VPMpc ${ }^{69}$. Other populations of PBN neurons project to the CeA, 
basolateral amygdala (BLA), BNST, LH and substantia innominata ${ }^{59,62,63,69-71}$. Some evidence also suggests that some cells collateralize to multiple forebrain regions, especially cells that project to both VPMpc and CeA. Differential organization of these projection cells across and within PBN subnuclei is also apparent. For example, the dl subnucleus contains LH, but not CeA or VPMpc-projecting neurons. A subset of the LHprojecting neurons here shows elevated activity to i.o. presentation of appetitive, but not aversive, taste stimuli. This subnucleus also receives concomitant input from the LH. Intriguing evidence from studies by Palmiter and colleagues show a large set of CeAprojection neurons found in the el subnucleus express calcitonin gene-related peptide (CGRP), respond to satiety cues, as well a variety of noxious stimuli (including the bitter taste of $\mathrm{QHCl}$ ), and may play a role in a general "threat detection" circuit ${ }^{72-74}$. Beyond this, there are few cellular markers of function, including taste, making cell populationspecific manipulation difficult. Other projections from the PBN target the periaqueductal grey, the midbrain dopaminergic (DAergic) areas (including ventral tegmental area [VTA]), and the ventral striatum. It is not clear if these projections have a gustatoryrelated function ${ }^{75}$.

As mentioned earlier, the PBN receives inputs primarily from the NST. An anterograde tracing study by Karimnamazi et al. (2002) ${ }^{76}$ examined inputs from either the rostral, gustatory NST (rNST), or the caudal, visceral NST (cNST). Terminals from rNST were found enriched at caudal levels of the PBN (especially in the wa), whereas those from cNST were found predominantly rostral. However, there was some degree of co-mingling of both types of input at every level, and in most subnuclei. In addition to ascending input from the NST, the PBN also receives input from spinal afferents, especially in more rostral portions of the el and dl subnuclei. The PBN also accepts ample descending input from many of the same forebrain regions discussed above. Studies with glutamate decarboxylase (GAD) transgenic mice (i.e., express fluorescent molecules on the GAD promoter) indicate the presence of many GABAergic fibers and terminals throughout the PBN, while there are relatively few GABAergic cell bodies (Boughter, unpublished). However, many PBN cells express GABA receptor subunits. This reflects descending inhibitory modulation from forebrain areas. Indeed, taste responses in the PBN can be inhibited via stimulation of the CeA or $\mathrm{LH}^{75,77}$. Anatomical studies also show that there is a substantial population of somatostatin+ cells in the CeA that coexpress GABA and project to the PBN ${ }^{78,79}$. 


\section{CHAPTER 3. THE NEUROBIOLOGY OF REWARD}

In this chapter, we turn our attention to the neurobiological evidence that supports the categorization of urge, pleasure, and displeasure into orthogonal dimensions of affect. The relationship of DA with urge has accumulated the most evidence and is elaborately explained. After detouring to the anatomy of the VTA, we will then venture into reviewing pleasure and displeasure, focusing on their relationship with feeding while also incorporating the mechanisms of urge.

\section{The Role of the Dopamine System in Reward}

A well-known group of DAergic nuclei are found in the midbrain that project to the striatum and other forebrain areas. Two pathways known as the nigrostriatal and mesocorticolimbic, whose function have been the subject of considerable debate, were alluded to earlier while introducing Schultz's reward prediction error and Berridge's incentive salience theories. The evidence provided below illustrates that the DA system's primary function is to elicit urge, albeit some of the nuclei involved also encode a degree of information about pleasure and displeasure. For further clarity, the reader is referred back to the section on reward in Chapter 1 and to specific manipulations of the neurobiology underlying affect as it pertains to feeding covered in Chapter 4. Working together and/or in opposition, these systems can collaborate or compete to ultimately produce approach, consumption, and avoidance behaviors (Figure 1-1).

\section{Dopamine Neurons Mediate Urge in Response to Rewards}

Midbrain DA neurons fire to novel, salient, and/or rewarding stimuli ${ }^{16,80-86}$, resulting in DA release in forebrain structures ${ }^{87,88}$. When the VTAs of rats are stimulated electrically, DA is released in the nucleus accumbens (NAc) ${ }^{88}$. Further, the elevation of DA and its metabolites were correlated with current intensity. A meta-analysis of studies using in vivo microdialysis to measure DA release in the NAc concluded that the typical peak after intravenous administration of cocaine (a highly rewarding stimulus and potently addictive drug) is slightly less than $300 \%$ of baseline $(2.39 \mathrm{nM})$ and that the dose-response is more correlated in the shell (NAcs) than core (NAcc) ${ }^{87}$.

After firing to a reward stimulus on the first exposure, midbrain DA neurons tend to shift the phasic onset of their firing to correlate with the onset of reward-predicting stimuli ${ }^{16,83,89,90}$. This shifting of phasic firing that occurs with subsequent exposures of the predictor-reward pair coincides with behavioral conditioning. After conditioning, midbrain DA neurons silence when reward fails to follow a reward predicting stimulus. From his electrophysiology experiments in primates, Schultz and his colleagues take this to mean midbrain DA neurons code for reward prediction error ${ }^{83,89}$. Confirmation in humans was done with functional magnetic resonance imaging (fMRI) ${ }^{83,91}$ and in mice with optogenetic single-cell identification of VTA DA neurons ${ }^{83,92}$. "Typically, 
dopaminergic (DA) neurons exhibit a highly stereotyped, short latency ( $<100 \mathrm{~ms}$ ), short duration $(\sim 100 \mathrm{~ms})$ population response to unpredicted stimuli in a variety of modalities that are salient by virtue of their novelty, intensity or reward value" ${ }^{80}$ (but see Freeman and Bunney, $1987^{81}$; Horvitz et al., $1997^{82}$; and Schultz, $1998^{16}$ ). Certainly, more can be said about the electrophysiology of midbrain DA neurons, but that level of detail is tangential to the point that DA mediates urge. Instead, the reader is referred to the numerous reviews and original research on this subject a few examples of which are cited here (Bruce Bean: Puopolo et al., $2007^{93}$; Lammel et al., $2008^{94}$; Fu-Ming Zhou: Ding et al., $2011^{95}$; Roeper, $2013^{96}$ ).

The aforementioned pervasive belief that DA release itself mediates pleasure has accumulated a wealth of empirical opposition. Depletion of DA with 6-hydroxydopamine (6-OHDA, which selectively destroys DAergic and noradrenergic neurons) lesions did not reduce positive facial reactions in taste reactivity tests, suggesting DA is not necessary for pleasure (or at least would not be necessary for activating the analogous circuitry which would be further processed in humans to elicit pleasure) ${ }^{10,17,97}$. DA neurons in monkeys cease to fire to juice rewards after the prediction is fully learned, but we assume the juice remains palatable ${ }^{10,89,98}$. Hedonic impact was not increased by activation of mesolimbic DA using several different methods, however, seeking and feeding was increased ${ }^{10,99-103}$. Human Parkinson's patients do not report lower subjective pleasure for sweet foods despite the disease's depletion of DA ${ }^{10,104}$. Parkinson's patients with DA dysregulation syndrome (DDS) compulsively request extra L-3,4dihydroxyphenylalanine (L-DOPA; a precursor of DA and a drug which when metabolized, increases DA levels) but do not report experiencing pleasure ${ }^{10,105}$. In fact, the dyskinesia, which the patients know results from excessive L-DOPA, is reported as extremely distressing. Rather than pleasure, DA release more likely elicits a general urge to act.

Two additional hypotheses about the function of the DA system remain the most competitive alternatives to the incentive salience hypothesis. The activation-sensorimotor hypothesis states that DA causes action generation, effort, movement, and general arousal or behavioral activation ${ }^{10,106-110}$. A considerable amount of evidence is emerging to support this; however, it cannot fully explain the phenomenon altered when one manipulates the DA system. A learning hypothesis derived from Schultz's work is less plausible (note that Schultz himself did not proffer this hypothesis). Anticipation of reward caused by conditioned stimuli that act as cues for rewarding unconditioned stimuli is associated with the activation of DA neurons ${ }^{10,16,111-114}$. Some investigators inferred from this that DA mediates the learning process of using cues to predict reward. Indeed, studies indicate that after conditioning DA in the NAc increases temporally with exposure to stimuli predictive of reward, but not when the reward is presented alone ${ }^{115-}$ ${ }^{118}$. However, this appears to be an encoding phenomenon rather than indicative of a learning process: DA deficient mice are still able to learn ${ }^{10,119}$ and hyperdopaminergic mice do not show higher learning (only increased motivation and locomotion) ${ }^{10,99,100,120-}$ ${ }^{122}$. Importantly, rats work harder for a reward when DA is activated ${ }^{10,123,124}$, indicating an increase in motivation. Generally, 'liking' activates 'wanting': 6-OHDA lesions (suppress 'wanting') and phasic benzodiazepine administration (stimulate 'liking') teases 
this apart ${ }^{10,17}$. After lesioning DA neurons in rats, the benzodiazepine, diazepam, was still able to enhance positive facial reactions in rats i.o. infused with palatable $0.3 \mathrm{M}$ sucrose.

Berridge and colleagues also devised an ingenious experiment to differentiate learning from incentive salience ${ }^{10}$. Berridge identified the ventral pallidum (VP) as an area receiving convergent input from all that DA is doing, allowing tests of his theory of incentive salience. Note that the temporal relationship of the conditioned stimuli (CSs) with the unconditioned stimulus (UCS) is important. "When two CSs in series (CS+1 followed by CS+2) always predict a sugar pellet reward (UCS), ventral pallidal neurons also gradually learn to shift firing forward, so that they eventually fire most to the first CS +2 tone" (Berridge, $2007{ }^{10}$ but see Tindell et al., $2004{ }^{125}$ ). Berridge explains another of this group's studies in detail regarding how they teased apart incentive salience from the rest of incentive salience theory ${ }^{102}$. They indicate $\mathrm{CS}+1$ is more predictive of the reward because it occurs the furthest before a reward. $\mathrm{CS}+2$ is more proximal because it occurs right before the reward is presented. Since the VP activity is ultimately highest for the $\mathrm{CS}+2$, this indicates DA is ultimately influencing a process for incentive salience (proximal rewards) not learning (predictive rewards). Interestingly, this coincides with human reports of intensifying experiences which reach a zenith just before selfadministration of addictive drugs (Hamilton, personal communication ${ }^{126}$ ).

\section{Dopamine and Displeasure}

Although most evidence of DA release is to rewarding stimuli, NAc DA release has also been shown to occur in response to aversive stimuli such as $\mathrm{QHCl}$, high $\mathrm{NaCl}$ concentrations, and red fox urine ${ }^{127}$. In addition to firing to pleasant stimuli and cues signaling pleasant stimuli, VTA DA neurons also fire in response to aversive stimuli such as footshock ${ }^{80}$ and air puffs ${ }^{92}$. The DA projections from the VTA to NAc are activated by stress (i.e., the experience of emotion within modalities associated with negative sentiments such as fear, anger, or sadness) ${ }^{128-138}$. Aversive and stressful events can cause DA release in the NAc ${ }^{86,139-143}$. Since both DA release and neuronal firing - the neurobiological hallmarks Berridge uses to indicate the attribute of incentive salience to pleasurable stimuli - may also occur in response to aversive stimuli, further assimilation suggests DA might ascribe a general attribute of salience to both incentives and disincentives. In this way, DA would function to elicit urge while separate systems mediating pleasure and displeasure determine the directionality of behavior (e.g., whether to approach or avoid). Perhaps Schultz agrees since when explaining the function of DA, he defines salience as a capacity of stimuli to elicit arousal, alertness, and attention ${ }^{144}$ and sets "incentive salience" off to the side because it depends on positive valence.

However, Schultz also states that although many studies have shown aversive stimuli can increase DA neuron firing and striatal DA release ${ }^{90,92,144-153}$, phasic depressions to aversive stimuli provide more evidence that the midbrain DA system does not function solely for reward. Moreover, Schultz indicates silencing of DA neurons may be a more powerful effector than activation and DA release ${ }^{144}$. Consider that the relative 
change (i.e., ratio) from 1 to near 0 approaches infinite, as opposed to the change from 1 to 3, which is only 3. Several reward neurons, including in the VTA, orbitofrontal cortex (OFC), prefrontal cortex (PFC), dorsal and ventral striatum, premotor cortex, and parietal cortex, show temporal discounting - that is, decreases in reward response as the time between the predictive stimulus and reward stimulus increases. In other words, phasic depressions occur not only to when predicted reward stimuli do not occur, but also to aversive stimuli or predictive cues of aversive stimuli ${ }^{80,90,92,142,144-152,154,155}$. Clearly, the VTA has a more multidimensional role than previously theorized, but more work is needed to explain these contradictory findings.

\section{The Ventral Tegmental Area}

So far the function of the VTA has been discussed as it naturally follows from a discussion of reward and DA; however, we will now divert our attention to its anatomy and then how that contributes to the VTA's function. The VTA is largely known for being 1 of 3 midbrain DAergic nuclei ${ }^{83}$. Activity of the DA neurons in this region results in DA release in the NAc, dorsal striatum, and PFC. Neurons within the VTA are responsive to taste stimuli, in line with the idea that reward value ascribed to the tastes of food relies on this nucleus. A wealth of investigation has been done into the anatomy and cytoarchitecture of this nucleus as well as its connectivity with other brain areas ${ }^{156,157 .}$

The VTA includes projections for 1 of up to 3 DAergic pathways that have been identified, depending on the source ${ }^{157}$ : A8 (mesocortical), A9 (nigrostriatal), and A10 (mesolimbic). A8, A9, and A10 specifically refer to the projections of DA neurons, whereas the nuclei and subnuclei, while marked by a primarily DAergic population, contain heterogeneous neuronal populations which also include glutamatergic and GABAergic cells (see Yetnikoff et al., $2014^{157}$ ). Sometimes A8 and A10 are combined and referred to as the mesocorticolimbic. A8 is a projection from the retrorubral field (RRF) to the cortex. A9 neurons project from the substantia nigra (SN) to the striatum. A10 somas are located in the VTA and project to the NAc. Two additional subdivisions of A10 are the A10dc (dorsal, caudal) which projects to the ventrolateral periaqueductal gray and A10rv (rostroventral) which projects into the supermammillary nucleus. Additional DAergic neurons project from the VTA to the hypothalamus, amygdala (AMY), lateral habenula, pallidum, and BNST ${ }^{143,158,159}$. The VTA can be broadly divided into 3 regions in mice: anterior (aVTA; from bregma -2.92 to $-3.28 \mathrm{~mm}$ in the mouse), posterior (pVTA; from bregma - 3.28 to $-3.80 \mathrm{~mm}$ ), and "tail" region (tVTA; from bregma - 3.80 to $-4.04 \mathrm{~mm}$; but also see below) ${ }^{160-167}$. Various VTA cell groups have been categorized as separate subnuclei ${ }^{156,157,168-171}$ by cytoarchitecture and projection. Proposed subnuclei of the VTA include the rostromedial tegmental area (RMTg), interfascicular nucleus (IF), caudal linear nucleus of the raphe (CLi), rostral linear nucleus of the raphe (RLi), pigmented parabrachial nucleus (PBP), and paranigral nucleus (PN) ${ }^{83,156,157,172}$. The PN is located ventromedially and is cell-dense, whereas the PBP is more lateral and dorsal. The IF, RLi, and CLi are midline structures ${ }^{157,167,169,172}$. The VTA can be divided by its medial and lateral regions (mVTA and IVTA, respectively) based on its forebrain projection patterns ${ }^{83,94,96,143,157,173-177}$. The medial 
VTA tends to project to the NAcc, medial NAcs, PFC, and BLA ${ }^{83,94,143,173-177}$. The lateral VTA tends to project to the lateral NAcs ${ }^{83,94,167,173-181}$. DA neurons are less dense in the RLi and CLi, which spreads into the paramedian mesopontine tegmentum ${ }^{157,167,182,183}$. VTA DA neurons have a differential latero-medial cellular co-expression of additional markers: amino acid decarboxylase (AADC), vesicular monoamine transporter 2 (VMAT2), DA transporter (DAT), DA D2 receptor (D2R), and vesicular glutamate transporter 2 (VGluT2) ${ }^{83,184}$. Calbindin-D $28 \mathrm{kD}$ expression occurs preferentially in DA neurons projecting to the NAcc ${ }^{157,185,186}$ and the RRF ${ }^{157,187}$.

The structure of VTA neurons (with long dendrites) combined with an enormously heterogeneous set of inputs allows the VTA to function as a likely integrator of information relevant to reward: "In the VTA itself, long dendrites enmeshed in major fiber bundles provide a morphological basis for a tremendous integration of different inputs" (Geisler and Zahm, $2005^{188}$ ). Inputs to the VTA include projections from the PFC, NAc, VP, CeA, BNST, LH, laterodorsal tegmental area (LDTg), and RMTg, among others $83,138,143,156,157,169,188-191$. VTA inputs are highly overlapping 157,188,191,192 - they are not discrete, but have difficult-to-define boundaries ${ }^{157,193}$.

\section{Rostromedial Tegmental Area and Non-DA VTA Neurons}

The debate over whether the RMTg is a VTA subnucleus (see Yetnikoff et al., $2014^{157}$ ) makes it worth additional discussion, especially as to how it might contribute to the function of the VTA and reward. It was first identified by Perrotti et al. (2005) ${ }^{182}$ who used the term "VTA tail" to describe a posterior region of the VTA in rats sparsely populated by tyrosine hydroxylase $(\mathrm{TH})$ neurons and found to express $\Delta$ FosB in GAD + neurons after chronic administration of psychostimulants. It later came to be known as the RMTg, located posterior to the VTA proper at $-3.9 \mathrm{~mm}$ posterior to bregma, $+/-0.9$ $\mathrm{mm}$ lateral to the midline, and $-3.6 \mathrm{~mm}$ ventral to the skull surface in mice ${ }^{194}$. It primarily contains GAD67-expressing GABAergic neurons; GAD+ cells are also present outside its perimeter ${ }^{166,195-198}$. Kaufling et al. (2009) ${ }^{166}$ and Jhou et al. (2009) ${ }^{197}$ appear to have located an anatomically similar (if not the same) area in rats and use either RMTg or the term "tail of the VTA". (Note that the "tail" region of the VTA mentioned above refers to the most posterior volume of the VTA, but these latter terms are alternative names which have been used for the RMTg.) The RMTg is known to receive input from the lateral habenula (LHb). Stamatakis and Stuber (2012) ${ }^{195}$ conducted a behavioral/system study in mice to determine the function of the LHb-RMTg circuit. Aversive stimuli activated a LHb excitatory input to RMTg neurons. Selective optogenetic stimulation of the LHb-RMTg circuit promoted avoidance.

Projections of the GABAergic neurons in RMTg, as well as other non-DA VTA projections, have been identified in mice ${ }^{198}$. Overall VTA projection neurons are $\sim 60-$ $65 \%$ DAergic, $\sim 30-35 \%$ GABAergic, and $\sim 2-5 \%$ glutamatergic ${ }^{157,198-201}$. However, VTA neurons are generally not systematically distributed across regions or subnuclei with respect to these neurotransmitters ${ }^{157}$. Taylor et al. (2014) ${ }^{198}$ characterized DA-independent efferents of mouse VTA, including to VP, lateral and magnocellular 
preoptic nuclei, $\mathrm{LH}, \mathrm{LHb}, \mathrm{CeA}$, mediodorsal thalamus, dorsal raphe, deep mesencephalic nuclei, PFC, and NAcs and NAcc. Some new technology reveals VTA input/output architecture some of which includes GABAergic neurons ${ }^{202}$.

VTA GABAergic neurons increased firing in response to rewarding and aversive stimuli ${ }^{92,148,198}$. However, activating VTA GABAergic neurons that project to the NAc did not modulate consumption of a reward ${ }^{83,203}$. More work is needed to determine what role the RMTg and GABAergic neurons in the VTA play in affect.

\section{The Neurobiology of Affect in Taste and Feeding}

Although DA is the neurotransmitter largely responsible for urge, other neurotransmitters/pathways may contribute pleasure and displeasure which add directionality to feeding behavior (i.e., approach, consume, avoid). A brief introduction to these relationships is covered here but excludes the work most relevant to the central focus of the experiments of this dissertation. Those neurobiological studies will be thoroughly covered in Chapter 4 (next).

The administration of opioid, cannabinoid, and benzodiazepine agonists to some brain areas may increase positive facial reactions to palatable food ${ }^{10}$. Opioid and benzodiazepines are reported by humans to elicit pleasure, and administration of the cannabinoids found in cannabis has a long-standing reputation for enhancing the palatability of food. Systemic $\Delta$-9-tetrahydrocannabinol (THC) administration increases hedonic reactions in response to intra-oral infusions of palatable stimuli ${ }^{204}$. The OFC, NAc, and interestingly, the PBN (sometimes referred to as "hedonic hotspots"), mostly via manipulation of these neurotransmitter systems, have been implicated in mediating pleasure ${ }^{205}$. The OFC mediates pleasure experience, valence, learning/memory, and in more posterior areas, it functions for sensory rewards such as taste ${ }^{205-209}$.

All three hot spots for pleasure in the brain use opioids ${ }^{205-209}$. Opioids in the VP also elicit 'liking' reactions ${ }^{209,210}$. The hedonic hotspot in the NAcs is rostral. Opioid activation via agonist microinjection in NAc amplifies positive facial reactions in taste reactivity tests, suggesting this may cause an increase in pleasure (or at least activating the analogous circuitry which would be further processed in humans to elicit pleasure) ${ }^{10}$. $\mathrm{Mu}$ opioid agonist [D-Ala ${ }^{2}, \mathrm{~N}-\mathrm{Me}-\mathrm{Phe}^{4}, \mathrm{Gly}^{5}$-ol]-enkephalin (DAMGO) in the rostrodorsal part of the medial NAcs caused a three-fold increase in positive taste reactions to sucrose ${ }^{208,210}$. Delta and kappa opioid receptor stimulation there also increases positive taste reactions ${ }^{206,210}$.

Systemic or intracerebroventricular (lateral) opioids increase food intake and positive taste reactions, and opioid antagonists block these effects ${ }^{57}$. The systemic opioid agonist morphine increased sucrose consumption, but had no effect on $\mathrm{QHCl}$ consumption ${ }^{211}$. Opioids administered systemically or to the lateral ventricles increased saccharin intake, but not neutral or aversive stimuli, and positive taste reactions in gastric fistulated animals. Beta-endorphins rise in blood and cerebrospinal fluid after palatable 
food consumption, suggesting mediation is via the hypothalamus (ventromedial, paraventricular nuclei), however, the hypothalamus may only increase appetite through energy regulation rather than palatability. The NTS contains mu-opioid receptors but not delta- or kappa-, suggesting this as another possible cite of action for opioid-mediated palatability enhancement. Park and Carr (1998) ${ }^{212}$ suggest the opioid system as the origin of the pleasure associated with food, however their work only confirms an increase in urge. Morphine injected into the VTA increased feeding, and naloxone blocked it. If this manipulation had also increased positive facial reactions, the intra-VTA opioid effect could be concluded to increase pleasure ${ }^{212}$, however, this has not been shown.

Palatability and consumption effects are shown to occur via GABA system manipulations. The systemic benzodiazepine agonist midazolam increased sucrose consumption, but had no effect on $\mathrm{QHCl}$ consumption ${ }^{211}$. GABA agonists increased food intake, especially palatable food intake, in gastric fistulated rats ${ }^{57}$. Intra-PBN midazolam (GABA) infusion increased food intake and hedonia-associated taste reactions to intraoral infusions to sucrose $+\mathrm{QHCl}^{10}$. Infusions into the PPT and NTS did not increase food intake, and taste reactivity was not tested for these areas. These effects suggest GABA manipulation impacts feeding through both pleasure and urge, including by specifically mediating effects through the PBN.

The NAc and PBN have also been implicated in mediating displeasure. Unlike the OFC and PBN, the NAc has been shown to act like a "keyboard" with an anteroposterior (AP) organization, wherein the anterior performs urge and pleasure functions, and the posterior, displeasure ${ }^{205}$. The el projects to the laterocapsular portion of the CeA (CeAlc) 73. The activation of this circuit decreases food intake, and inhibition prevents suppression of appetite. The el also projects to BNST, LH, medial thalamus, and the dorsal PBN. The el was not found to project to the arcuate or paraventricular nuclei of the hypothalamus. As mentioned above, neurons especially responsive to posterior oral stimulation have been found in the rat el ${ }^{69,213,214}$. Fos expression was high in rat el when they were stimulated with intra-oral, but not intra-gastric, QHCl solution ${ }^{66,68,215}$. Two other non-taste functions of el neurons include salt and nociceptive responses. 


\section{CHAPTER 4. THE TASTE/REWARD INTERFACE OF THE PBN AND VTA}

\section{Direct and Indirect Connectivity between Taste and Reward Areas}

For taste, experiments conducted by Hajnal and Norgren (2005) ${ }^{216}$ first showed, using a sham feeding preparation, that the taste of sucrose alone led to DA release in the NAc. They also found that this release was greatly attenuated when the PBN was lesioned, suggesting that the PBN plays a key role in this process. However, the exact nature of this connectivity was not known, despite evidence that tracing studies that some PBN neurons, including those characterized as glutamatergic, project directly to the VTA ${ }^{80,188}$ (Saites et al., 2015, poster ${ }^{217}$ ). The nature of this projection, and its potential role in taste/reward/intake, is a topic of study of this dissertation. We hypothesized that taste information communicated from the PBN to the VTA may be used to generate urge, priming or causing the animal to approach and consume. If the PBN projection to the VTA is excitatory and its terminals synapse on DA neurons there, exposure to rewarding taste stimuli might activate this circuit and cause DA release. As explained in Chapter 3 , VTA DA neurons shift their activity toward proximal cues, and this increases the urge associated with those cues.

It is possible that taste information in the PBN could take a more direct or an indirect approach to influencing striatal DA release. Li et al. (2012) ${ }^{75}$ showed via in vivo physiology that a subpopulation of taste-responsive PBN cells projects directly to the NAcs in hamsters; while the mechanistic function of this projection is uncertain, they also showed that a reciprocal NAc to PBN projection is inhibitory with regard to taste responses. Retrograde tracing data from our laboratory, however, suggested that this is a rather small projection relative to others, at least in mice, with only a handful of cells projecting to NAc located in taste-related areas of the PBN (Figure 4-1; Saites et al., 2016 , poster ${ }^{218}$ ). As mentioned above, the PBN also projects robustly to areas with known involvement in feeding and associative learning such as the CeA, BLA, and LH 59,138,219-221. In turn, retrograde and anterograde tracing studies revealed both CeA-to-VTA and LH-to-VTA projections ${ }^{188}$ in rats ${ }^{189,222-224}$. Evidence suggests the BLA also projects to the $\mathrm{CeA} 43,225,226$, which would indirectly connect the BLA to the VTA. The BLA and CeA have reciprocal connectivity with the gustatory brainstem (NST and PBN), and the $\mathrm{BLA}$ has reciprocal connections with the $\mathrm{GC}^{227-231}$. Electrical stimulation of the BLA increases neuronal activity of the VTA ${ }^{138,219-221}$. Orexin (OX) and melanin concentrating hormone $(\mathrm{MCH})$ neurons in the LH project to the VTA ${ }^{222-224}$. LH-projecting PBN neurons show elevated activity to i.o. gustatory stimuli ${ }^{59}$. Collectively, these PBN to limbic to VTA circuits are not as direct as connections from the PBN to the NAc or VTA, but this anatomy clearly supports the possibility that multiple, parallel taste-responsive brainstem-to-midbrain/forebrain circuits could be simultaneously regulating DA release, reward, urge, and feeding. 

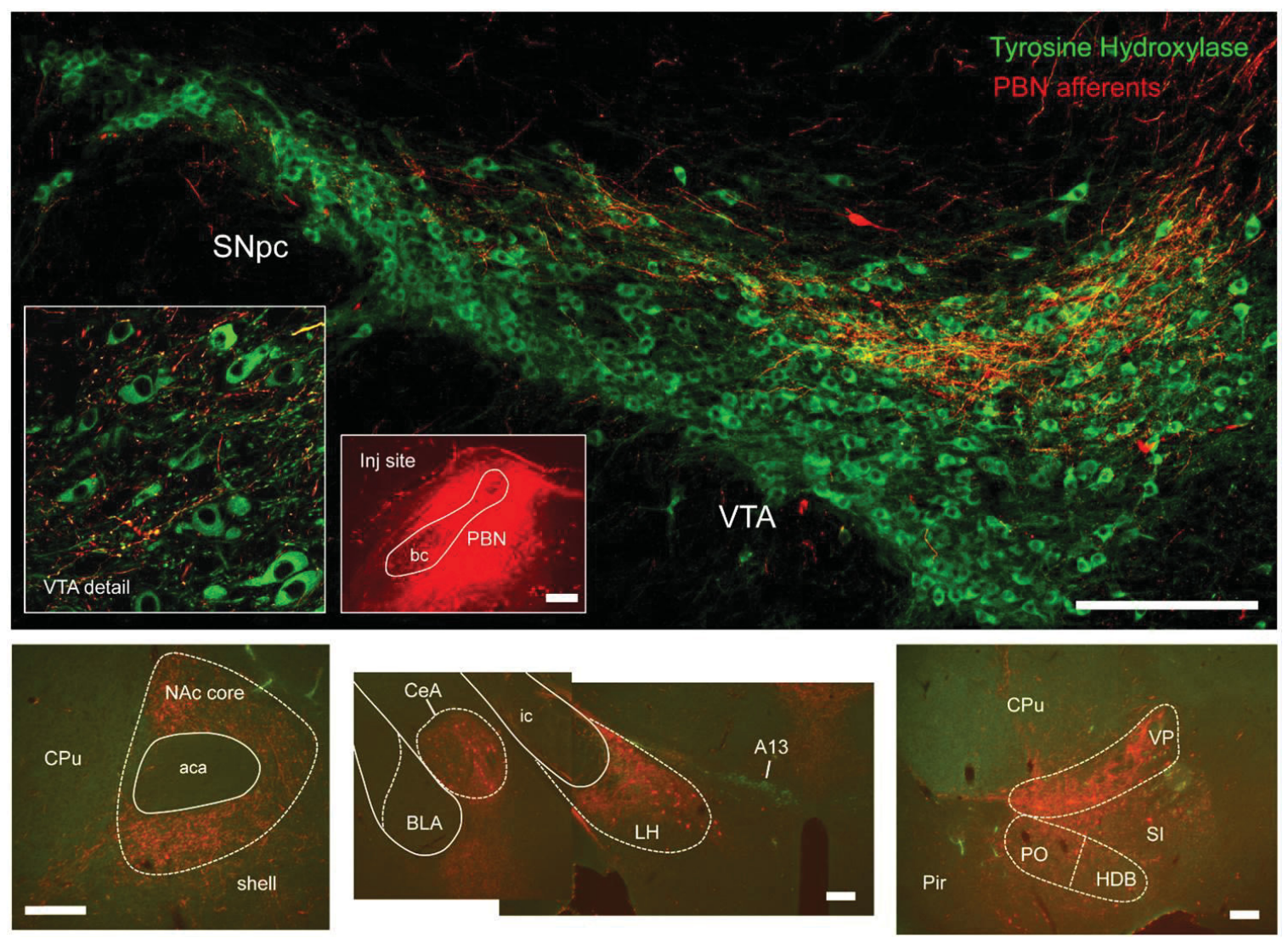

Figure 4-1. Parabrachial nucleus (PBN) fibers and varicosities in midbrain and limbic regions.

Top: Dopaminergic cells (green) are indicated by tyrosine hydroxylase (TH) labeling; higher power image (lower left) shows apposition of what may be PBN projection neuron terminals with DA neuron dendrites; inset (lower middle) shows the injection site in the PBN. Bottom: Nucleus accumbens (NAc), central nucleus of the amygdala (CeA), lateral hypothalamus (LH), and ventral pallidum (VP) were positive for PBN fibers; A13 of the zona incerta was an additional area labeled positive for TH. Substantia nigra pars compacta, SNpc; ventral tegmental area, VTA; brachium conjunctivum, BC; caudoputamen, $\mathrm{Cpu}$; anterior part of the anterior commissure, aca; basolateral nucleus of the amygdala, BLA; internal capsule, ic; piriform cortex, Pir; preoptic nucleus, PO; horizontal band of Broca, HDB; substantia innominata, SI. Scale bar $=200 \mu \mathrm{m}$. 


\section{PBN and VTA in the Affect of Taste}

In Chapter 3, we saw that several neurotransmitter systems throughout the brain can contribute to the affect of taste, which in turn, may influence feeding. Whereas DA tends to influence the dimension of urge, opioid, GABA, and cannabinoid receptor modulation may alter pleasure and displeasure. As noted, these systems work throughout the brain, but this section reviews the evidence specific to the PBN and VTA.

Evidence suggests opioids in the PBN may increase both the pleasure of, and urge to consume, palatable tastes, indicating a role in priming or causing of feeding. The PBN contains a high density of opioid receptors ${ }^{57,212,232}$. Specifically, the lateral PBN contains mu-opioid receptors, suggesting this as a possible site of action of systemic opioid action and to be necessary and sufficient to mediate food intake and increase positive facial reactions ${ }^{57}$. Intra-PBN naltrexone decreases feeding ${ }^{212,233}$ and morphine increases saccharin preference in animals whose preference was lost due to LH lesioning ${ }^{212,234}$.

The GABA system in the PBN may also increase pleasure and urge to palatable tastes. The $\mathrm{GABA}_{\mathrm{A}}$ agonist, midazolam, increases food intake and palatable taste reactivity when intraperitoneal (i.p.) injected, indicating GABA's effect is mediated by $\mathrm{GABA}_{\mathrm{A}}$ receptors ${ }^{57,235}$. Administration of GABA agonists also increased palatable food intake in gastric fistulated rats ${ }^{57,236}$, meaning this effect occurs independently of postingestional input (i.e., taste specific). Intra-PBN midazolam (GABA) infusion increased food intake and hedonia-associated taste reactions to intra-oral infusions of a bittersweet $7 \%$ sucrose $+0.01 \%$ QHCl solution ${ }^{10,237,238}$.

The processes by which DA may regulate feeding and interact with other neurotransmitter systems (and reward aspects) remain unclear. Examination of forebrain DA release and midbrain activity suggests the VTA causes urge or drive in response to stimuli with extreme taste hedonics regardless of valence. Stimulating rats with palatable foods elicits activity in the VTA ${ }^{212}$. It also causes DA release in the NAc and medial PFC (mPFC) ${ }^{127,239}$. However, DA release in the NAc and PFC occurs in response to both sweet (i.e., appetitive) and bitter (i.e., repulsive) substances ${ }^{127}$. The pathway from the ventral striatum (approximately the same structure as NAc) to the VP (the downstream output of which was shown by Berridge to indicate salience) communicates taste but not caloric information ${ }^{240}$; thus, taste salience information is likely to be communicated by the VTA-to-NAc pathway (also see next two sections on post-ingestive and taste input and manipulating VTA output). Interestingly, activating VTA DA neurons decreases food intake $^{241}$, and activating the VTA-to-NAc projection recapitulates the effects of this manipulation better than activating projections to either the PFC or AMY. This suggests the taste salience information carried by the VTA-to-NAc may lean towards negative. Habituation of DA release to food rewards occurs in the NAcs, but not in mPFC and NAcc ${ }^{239}$. Chronic, but not acute morphine, can sensitize the NAcs so that habituation of DA release to food reward no longer occurs. This pattern of DA release habituation in the NAc to food rewards may occur in part due to effects in the VTA DA neurons. Pleasurable and urge effects on feeding of opioids are at least partially mediated via the 
VTA because Fos expression increases in the VTA of rats in response to a palatable meal can be blocked with opioid antagonists such as naltrexone ${ }^{212}$.

\section{Post-ingestive and Taste Interactions}

That flavor-the composition of odor and taste-alone can elicit pleasure begs the question of Why do we swallow food? Indeed, if we are trying to prevent weight gain or even lose weight, Why would we not allow ourselves to experience the flavor and then spit the food back out? It stands to reason that something about swallowing or the "feeling of fullness" is also rewarding. It may be that viscerosensation communicates in its own way to pleasure centers. Furthermore, since post-ingestive effects (i.e., calories) are also likely drivers of appetite, various controls must be implemented to ensure consumption effects are in fact due to taste and palatability (addressed in methods sections throughout this dissertation).

Responsiveness in the PBN to intragastric (i.g.) stimulation differs from i.o. ${ }^{68}$. Fos expression was measured in the area postrema, NST, and PBN to differentiate these effects. QHCl (bitter-aversive), sucrose (sweet-preferred-caloric), saccharin (sweetpreferred-non-caloric), and water (control) were used to stimulate. I.g. and sucrose stimulation elicited more Fos expression in general, but water did not. Differential Fos expression of the brainstem areas occurred on the basis of i.g. versus i.o. and depending on the taste stimulus, including caloric versus non-caloric and preferred versus aversive. Besides in the PBN, the path visceral information takes centrally parallels that of taste, except for at least one difference in that the cNST (rather than rNST) is responsive $57,242,243$.

Additional work has shown that viscerosensation and the transmission of caloric signals may be just as effective at driving consumption behaviors as taste. Trpm5 -/- mice which are blind to sweet taste (see Chapter 6 methods) ${ }^{244,245}$ continue to develop a preference to the caloric sweetener, sucrose, but not to the non-caloric sucralose ${ }^{246}$. Further, sucrose, but not sucralose, caused significant NAc DA release in trpm5 -/- mice. The authors suggest these effects are due to post-ingestive feedback, which remain intact despite the disruption to the taste pathways. Moreover, separate circuits at levels higher than the PBN and VTA were implicated in the communication of hedonic and nutritional information about sugar 240 .

\section{Effects of Manipulating VTA Function on Feeding}

Manipulating VTA neurons with lesioning and designer receptors exclusively activated by designer drugs (DREADDs) activation produces mixed results. Bilateral lesions induced electrolytically in the VTA of rats had a negative effect on sucrose consumption, but not of other non-palatable fluids ${ }^{211}$. Although activating VTA DA neurons with DREADDs had little effect on feeding latency, it decreased average meal size, increased feeding frequency, and decreased total food intake ${ }^{241}$. The effects of this 
manipulation appeared to be primarily dependent on the VTA neurons that project to the NAc. Although activating the VTA-NAc projection did not change total food intake, it decreased average meal size and increased feeding frequency. Neither VTA-PFC, nor VTA-AMY projections had significant effects on these factors. That loss-of-function and gain-of-function experiments yielded similar effects on feeding indicates both the VTA contains mechanisms for change in both directions and more targeted approaches are necessary for teasing apart how the VTA modulates feeding.

Rats given subcutaneous morphine (opioid) or i.p. midazolam (GABA agonist) increased sucrose consumption, but this was blocked with bilateral VTA lesioning of DA neurons with 6-OHDA ${ }^{211}$. Opioid agonists injected into the VTA increased feeding ${ }^{212,247}$ and had positive reinforcing effects $212,248,249$. I.p. opioid antagonists blocked the increase in feeding ${ }^{250}$. Activation of neurons in VTA by meal or meal-paired environment was decreased by naltrexone, possibly due to activation of opponent neurons by naltrexone 212. This evidence suggests the intra-VTA opioid effects are on urge, however, it seems more likely to occur via an effect on pleasure. Taste reactivity would need to be tested in conjunction with intra-VTA opioid manipulation to verify this.

VTA GABA effects interact with opioids and appear opposite for feeding relative to classical versus operant conditioning. GABA agonists administered directly to the

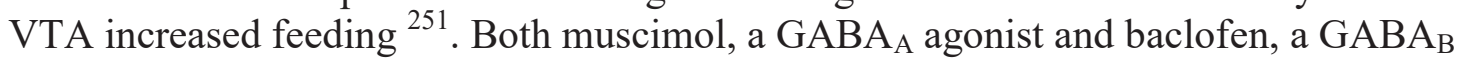
agonist, increased feeding, and the baclofen-induced increase was blocked by the $\mathrm{GABA}_{\mathrm{B}}$ antagonist, saclofen. Naltrexone had no effect on the increased feeding induced by intra-VTA muscimol, but it blocks the baclofen-induced increase ${ }^{252}$. Interestingly, intra-VTA GABA agonists (combined muscimol and baclofen) inhibited lever pressing after rats were trained to respond to a CS+ for food pellets ${ }^{253}$.

Results in experiments in which the VTA is unilaterally manipulated indicate there is a crossing over in the transmission from the VTA to areas more directly responsible for feeding. When one side of the VTA of rats was lesioned and the contralateral side stimulated, the increase in Fos expression was amplified compared to the increase seen in the unlesioned but unilaterally stimulated group ${ }^{162}$. Similarly, a shortened latency to eat in the unlesioned, unilaterally stimulated group was amplified in the group with a contralateral VTA lesion. Unilaterally lesioned rats that received no VTA stimulation showed no changes in Fos or behavior. This indicated the lesions had suppressed the inhibition of afferent brain areas to the VTA ${ }^{162}$. Further, unilateral block of excitatory input via N-methyl-D-aspartate (NMDA) receptors and current into the contralateral VTA led to a shortened feeding latency ${ }^{254}$, suggesting at least the feeding effect is mediated by glutamate.

\section{Aims and Experiments}

Since earlier studies and data collected in our lab show that PBN neurons project to the VTA ${ }^{80,188}$, and lesioning the PBN attenuates taste-elicited release of DA in the NAc ${ }^{216}$, we hypothesized this connection plays a crucial role in the control of feeding, 
especially with regard to the processing of both appetitive and aversive stimuli, and the relationship of this processing to classical reward circuitry. We therefore utilized a number of neuroanatomical and behavioral techniques to probe taste and intake-related activity in the PBN, VTA, and the PBN-to-VTA circuit. The overarching goal was therefore to contribute to a comprehensive understanding of feeding neurobiology via improving the knowledge about these brainstem nuclei. The working model for the neurobiology of taste-mediated reward for this dissertation is illustrated in Figure 4-2.

\section{Analyze the PBN Response in Relation to Taste Valence and Characterize its Projection to the VTA}

We used two experiments to look for differences in the expression of the IEG cFos in PBN neurons. In this and all other c-Fos experiments in this dissertation, c-Fos expression is measured with the use of a polyclonal antibody to the Fos protein; therefore, the term "c-Fos-like immunoreactivity" (FLI) is henceforth used. One experiment tested the hypothesis that FLI in the PBN is elevated specifically in response to taste stimuli by using the trpm 5 -/- mutant, a mouse model based on the C57BL/6J (B6) phenotype but lacking the ability to taste sweet and bitter stimuli. We examined whether FLI within PBN subnuclei and at varied rostral-caudal levels differs among palatable and aversive stimuli. We expected trpm 5 -/- mice to have lower FLI in the PBN than B6 after taste stimulation specifically in areas responsible for relaying taste information.

The second experiment retrogradely-labeled PBN neurons in B6 mice by their projection to either the VTA or gustatory thalamus (parvicellular region of the venteroposteromedial; VPMpc) and measured their corresponding FLI to palatable and aversive stimuli. Regional and functional comparisons of the PBN projections to either VTA or VPMpc were made to assess whether palatable or aversive taste stimuli preferentially elevated activity in one pathway or the other. Retrograde tracing was expected to reveal regional and functional differences in the PBN projections to either ventral (VTA) or thalamocortical pathways (reflecting reward and sensory processing, respectively). The intention was to further make functional differences apparent by combining tracing with sucrose- or QHCl -elicited FLI.

\section{Determine the Location and Morphology of VTA Neurons Responsive to Taste Stimuli with Varied Valence}

Again, we conducted two experiments, except this time to examine the VTA. A continuation from the first PBN experiment, we observed taste-evoked FLI in the aVTA, pVTA, and tVTA. Trpm5 -/- mice were expected to have lower FLI in the VTA than B6 mice after taste stimulation, highlighting specific areas for relaying taste information. 


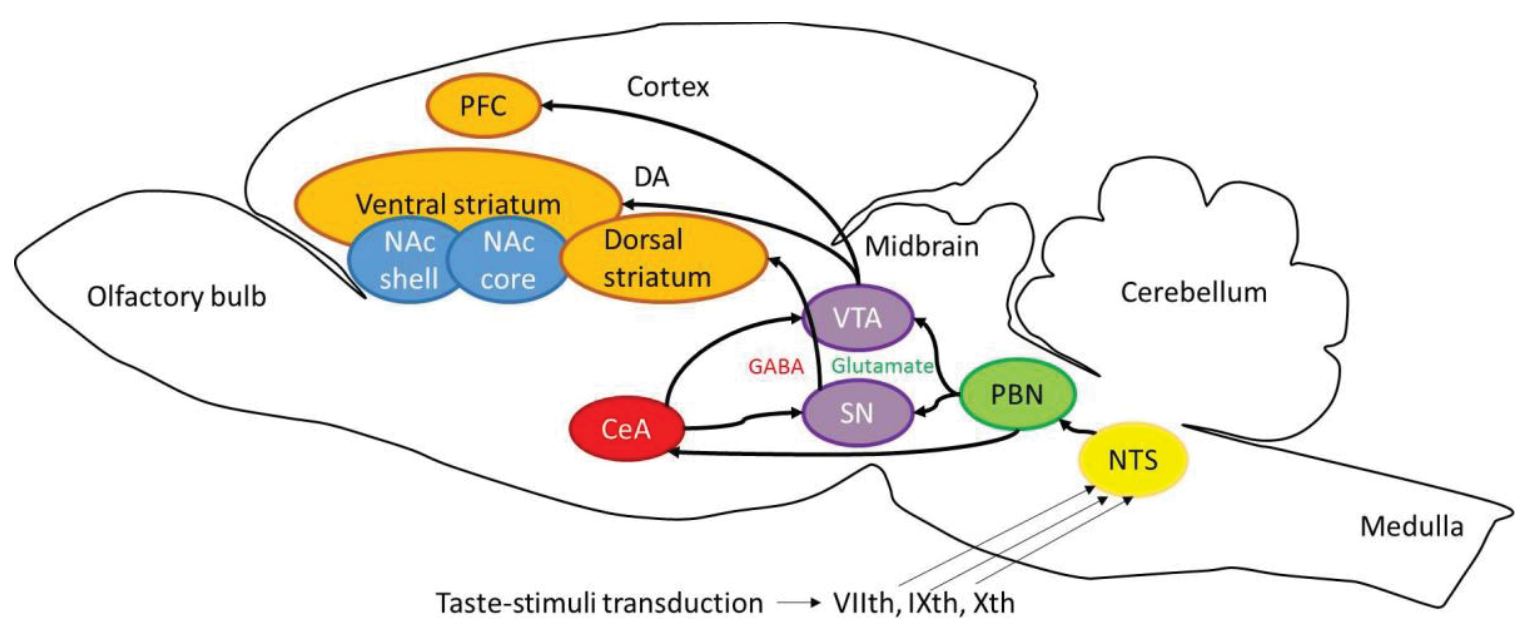

Figure 4-2. Taste reward pathways.

Taste information enters the central nervous system via cranial nerves VIIth, IXth, and Xth. After being relayed through the nucleus tractus solitarius (NTS), it travels to the parabrachial nucleus (PBN) in the pons. The PBN, along with two additional nuclei in the brainstem to which it projects, the ventral tegmental area (VTA) and substantia nigra $(\mathrm{SN})$, integrate sensory information to generate affect. The PBN also projects to the CeA which projects back to the VTA and SN, two dopaminergic nuclei located in the midbrain. The midbrain DA projections to forebrain areas, including the ventral striatum (composed of the nucleus accumbens [NAc] shell and core), dorsal striatum, and prefrontal cortex (PFC), which begin motor circuits, complete this pathway. 
The most common neurotransmitters released by VTA neurons are DA and GABA $^{198}$; these neurons were identified by their fluorescently labeled synthetic enzymes, TH and GAD67, respectively. Taste stimuli were delivered as in previous experiments. FLI, TH, and GAD67 labeled neurons (and double-labeling) were counted in the VTA delineated by its regions and subnuclei. This was expected to elucidate any differential in VTA activity elicited by palatable or aversive taste stimuli.

\section{Modulate Feeding by Manipulating the VTA with DREADDs and Drugs}

We targeted the VTA using two different methods to determine if feeding could be altered, presumably on the basis of effects on taste and/or reward circuitry. The first experiment aimed to measure the potential effects of activating the PBN-to-VTA circuit on taste-mediated feeding behavior. A two-part vector microinjection selected only the VTA-projecting PBN neurons for expression of excitatory DREADDs. Clozapine-Noxide (CNO) administration activated only the PBN-to-VTA circuit, allowing measurement of any feeding behavior it mediates. A behavioral task performed using a lickometer included measuring licks and trials initiated by mice after activation of the PBN-VTA circuit. Licked stimuli were varied on the basis of both taste valence and caloric content. Although activating this circuit was expected to affect feeding, the specific effects were challenging to predict due to uncertainty about whether PBN-VTA projections targeted only DA neurons or if VTA GABA neurons received input to the extent it would overwhelmingly oppose direct input to DA neurons.

Due to inconclusive preliminary results in the PBN-VTA experiment, we postponed it in lieu of one which targeted the VTA directly. We pharmacologically inhibited the VTA with a GABA agonist while using the same behavioral paradigm and stimuli variation described above. We expected to see an increase in consumption and to determine if the VTA was a necessary cause of any feeding effects. 


\section{CHAPTER 5. GENERAL METHODOLOGY}

The experiments of this dissertation continued work being done in the Boughter lab to elucidate the role of PBN connectivity in reward and feeding. Preliminary data from immunohistochemistry, tract tracing, genetic mouse mutants, and behavioral experiments indicated a functional connection for taste, reward, and feeding existed between the PBN and VTA. We continued the collection of this data in part by using the following general methods. Methodology specific to each experiment will be explained in Chapters 6 through 8 .

\section{Animals}

These studies used 104 male and female mice, including 74 B6, 18 trpm 5 -/knockout mice (KO), and 12 CB6-Tg(Gad1-EGFP)G42 mice (GAD67-GFP). The sample sizes chosen were the same or similar to those of relevant published reports, including from the Boughter lab ${ }^{59,67,68,80,198,255-262}$. Additional mice were excluded for reasons noted in the following chapters. Mice were housed in the University of Tennessee Health Science Center (UTHSC) Lab Animal Care Unit with controlled humidity and temperature on a $12 \mathrm{~h} \mathrm{light/dark} \mathrm{cycle} \mathrm{(lights} \mathrm{on} \mathrm{at} 0600$ and off at $1800 \mathrm{~h}$ ). Mice were fed a standard diet of dry pellet chow (22/5 rodent diet, Harlan Teklad, Madison, WI, USA). All studies were approved by the UTHSC Animal Care and Use Committee prior to experiments, and mice were handled in accordance with the National Institute of Health Guide for Care and Use of Laboratory Animals (NIH Publications No. 80-23), revised 1996.

\section{Intraoral Cannula Implant}

Mice were anesthetized with an i.p. injection of $0.04 \mu \mathrm{L}$ of ketamine/xylazine (approximately 100/10 mL/kg; Fort Dodge Animal Health, Fort Dodge, IA, USA and Lloyd Laboratories, Shenandoah, IA, USA). Additional injections of 0.01 or $0.02 \mu \mathrm{L}$ of ketamine/xylazine were given until the mouse passed the toe pinch test (i.e., no flinching) and throughout the procedure as needed if the mouse appeared to be waking up (i.e., vocalizing, ambulating, whisking). To apply taste stimuli directly to the oral cavity (i.e., i.o.), a piece of polyethylene (PE 50; Intramedic, Sparks, MD, USA) tubing approximately $13 \mathrm{~cm}$ long was tunneled through the buccal mucosa along the lateral surface of the skull guided by a $25 \mathrm{~g}$ needle. The cannula exited through a $\sim 0.5 \mathrm{~cm}$ hole made in the mouse's scalp. Flaring one end of the cannula (to prevent its recession into the buccal mucosa), a screw in the skull (No.19010-10; Fine Scientific Tools, Foster City, CA, USA), and dental acrylic (Unifast Trad; GC Corporation, Tokyo, Japan) were used to fix the length $(\sim 2 \mathrm{~cm})$ of the cannula between the oral cavity and top of the head. After the procedure, connecting the cannula to additional tubing, a syringe, and syringe pump (model 341A; Sage Instruments, Cambridge, MA, USA) allowed volume- and ratecontrolled injection of taste stimuli into the oral cavity. Cutting the cannula's end at an 
angle eased its entry into the larger connecting tube (PE 90; Intramedic, Sparks, MD, USA); the inner diameter of the connecting tube was $\sim 0.1 \mathrm{~mm}$ smaller than the outer diameter of the cannula, sealing the connection. Occasionally, mice chewed the cannula, requiring additional shortening to prevent leakage.

\section{Adaptation and Tastant Stimulation}

To habituate mice to extraneous stimuli before collecting FLI data, they were trained for 3 or 4 days by administering distilled water (DW) through the cannula at the same volume and rate to be used in testing. Training generally occurred in the same chamber and at the same time as that of testing (between 1000 and $1300 \mathrm{~h}$ ). Mice were provided access to food and water ad libitum during training but were deprived $20 \mathrm{~h}$ prior to testing to promote sampling; this short deprivation period does not cause significant weight loss. On test day, taste stimuli were delivered i.o. for $15 \mathrm{~min}$ at a rate of 0.10 $\mathrm{mL} / \mathrm{min}$ (total volume $=1.5 \mathrm{~mL}$ ). Taste stimuli were $3 \mathrm{mM} \mathrm{QHCl}$ and 0.5 and $1 \mathrm{M}$ sucrose prepared fresh using reagent grade chemicals (Sigma-Aldrich, St. Louis, MO, USA) dissolved in DW; DW was used as a control stimulus (Table 5-1). A period of $2 \mathrm{~h}$ separated the beginning of stimulation from perfusion onset. Mice remained deprived during this wait period.

\section{Histology}

Mice were anesthetized with $0.4 \mathrm{~mL}$ of $25 \%$ urethane. Their thoracic cavities were opened to expose their hearts with a Chevron incision beginning directly below the xyphoid process moving laterally followed by two paramedian incisions anteriorly from the outer edges of the first cut. A needle was inserted into their left ventricles, and a slit was cut in their right atria for exsanguination. Mice were transcardially perfused with $25 \mathrm{~mL}$ of $0.1 \mathrm{M}$ phosphate buffered saline (PBS), followed by $\sim 50 \mathrm{~mL}$ of chilled $(\sim 4$ $\left.{ }^{\circ} \mathrm{C}\right) 0.4 \%$ paraformaldehyde in $0.1 \mathrm{M}$ phosphate buffer (PB; $0.4 \%$ PFA). Brains were carefully removed and placed in jars with $0.4 \%$ PFA for at least $24 \mathrm{~h}$ in $4{ }^{\circ} \mathrm{C}$, followed by a $20 / 10 \%$ sucrose/glycerol solution in $0.1 \mathrm{M} \mathrm{PB}$ (cryoprotectant) for at least $24 \mathrm{~h}$ in $4{ }^{\circ} \mathrm{C}$. Brains were frozen with dry ice and sliced into $40 \mu \mathrm{m}$ thick coronal sections using a freezing microtome (860; American Optical Company, Buffalo, NY, USA). Brains were marked to identify left/right and ipsi/contra side. Sections were divided into two sets and kept in rostrocaudal order. One set of sections were Nissl-stained with cresyl violet (CV). When immunohistochemical (IHC) procedures were used, they preceded sections being mounted. Sections were mounted in rostrocaudal order by rolling them with a paint brush on to silane-coated slides (P4981-001; Thermo Scientific, Waltham, MA, USA). Slides were dried for at least 24 hours before CV staining or coverslipping. A mountant/xylazine (mountant was DPX for histology; Fluka, Milwaukee, WI, USA) mixture (1:1) was used to fix coverslips (except for CV, which used pure DPX). Coverslipped slides were dried at least $24 \mathrm{~h}$ prior to imaging. 
Table 5-1. Stimuli used in anatomical, physiological, and behavioral studies.

\begin{tabular}{ccc}
\hline & \multicolumn{2}{c}{ Concentrations (M) } \\
\cline { 2 - 3 } Tastant & Experiments 6.1, 6.2, 7.1, 7.2 & Experiments 8.1, 8.2 \\
\hline Sucrose & $1,0.5$ & 0.3 \\
QHCl & 0.003 & 0.0001 \\
Sucralose & & 0.003 \\
Water* & N/A & N/A \\
\hline
\end{tabular}

* Negative control used in all experiments. 


\section{Immunohistochemistry}

Except for the nickel-enhanced 3,3'-diaminobenzidine $\mathrm{HCl}$ (DAB) IHC procedure (Chapters 6 and 7), immunohistochemistry protocols followed the same 3 basic steps of binding the target molecule with primary antibodies, binding the primary antibodies with biotinylated secondary antibodies, and binding the secondary antibodies with fluorophore-conjugated streptavidin. However, binding with secondary antibodies and fluorophore-conjugated streptavidin steps were repeated twice to label two separate target molecules (except for mCherry, the antibody for which was already conjugated to fluorophore [Chapter 8]). Sections were washed with 0.02 M PBS beforehand, in between each step (except between blocking and antibody incubation), and after the last step. Blocking used 3\% serum derived from the same animal in which secondary antibody was harvested and $0.5 \%$ triton-X 100 in $0.02 \mathrm{M}$ PBS.

\section{Imaging}

Brightfield, fluorescent, and confocal microscopy were used to observe sections and collect images as data and for cell counting. Images were collected using: 1) a Leica (DMRXA2, Leica Microsystems, Bannockburn, IL, USA) episcopic-fluorescence microscope equipped with a digital camera (Hamamatsu ORCA-ER, Hamamatsu Corp., Shizuoka, Japan) and imaging software (SimplePCI, Hamamatsu Corp., Shizuoka, Japan) and, 2) a confocal microscope (Zeiss 710, Carl Zeiss, Thornwood, NY) and imaging software (ZEN, ZEISS, Thornwood, NY). 


\section{CHAPTER 6. TASTE-EVOKED ACTIVITY IN PBN NEURONS INCLUDING PROJECTIONS TO EITHER THE VTA OR GUSTATORY THALAMUS}

Physiological and neuroanatomical approaches have established that a substantial population of neurons are responsive to gustatory stimuli in the mouse PBN, and emerging evidence suggests a direct taste-reward pathway originates in this brainstem nucleus. However, much remains unknown about the selectivity and responsiveness of PBN cells that project directly to the VTA. Stimulus-evoked activity of neurons (including by taste stimuli) in the PBN has been frequently assayed using expression of cFos (or FLI) ${ }^{66,73,212,263}$. An advantage of this method, which involves immunohistochemical and microscopic analysis, is that it allows quantification of the entire population of reactive cells throughout a brain region. Previous studies in rats and mice show that taste stimuli of different taste qualities tend to evoke distinct patterns in the PBN. Additionally, some evidence indicates particular tastants preferentially stimulate certain classes of projection cells. Using a combination of retrograde tracing and taste-evoked FLI, Tokita et al. (2014) ${ }^{59}$ showed that a sweet-tasting, caloric stimulus (sucrose) preferentially elevated activity in LH-projecting PBN neurons relative to other stimuli. Palmiter and colleagues (Carter et al., $2013^{73}$ ) showed that $\mathrm{QHCl}$ selectively activates CGRP+, AMY-projection neurons in the el.

In most taste-IEG experiments, taste stimuli are presented in awake, freely moving animals through i.o. cannulas; this ensures equivalent stimulation among tastants, even to those that are hedonically negative such as QHCl. However, mice may still consume stimuli during this procedure. Although it is assumed that IEG expression based on i.o. stimulation is predominantly orosensory in nature (see Yamamoto et al., $1994{ }^{66}$ ), it is possible that some amount of it is also based on post-oral or visceral processing, especially for preferred stimuli. Therefore, in Experiment 6.1 we examined i.o. evoked FLI across the PBN to taste stimuli regarded as canonical examples of hedonically pleasurable (sucrose), displeasurable $(\mathrm{QHCl})$, and neutral (water) in both $\mathrm{B} 6$ and "tasteblind" KO mice. This approach should provide insight into the "type" of information potentially being relayed to upstream brain areas.

In Experiment 6.2 we used retrograde tracers injected into either the VTA or gustatory thalamus (VPMpc) in combination with i.o. evoked FLI to the same stimuli, to see if there is stimulus specificity in terms of activity of projection neurons. The VPMpc was chosen as a second projection target to provide a contrast to the VTA on several fronts. While we reasoned that a palatable stimulus would preferentially elicit activity in VTA-projection neurons due to our hypothesized role of this projection in taste-reward processing, we did not predict specificity in activity of thalamic projection neurons. As mentioned earlier, the VPMpc serves as the primary relay to $\mathrm{GC}$, and this projection has been historically considered to play the primary role in conscious processing and discrimination of taste quality. Therefore, it seems reasonable to predict that stimuli of all qualities and valences should elicit activity in VPMpc-projecting cells. Another reason to compare the VTA projection with the VPMpc projection is to gain possible insight into PBN subnuclear function, i.e., Do specific PBN subnuclei project to either brain area? 
and Do individual PBN neurons collateralize to both VTA and VPMpc, indicating input of "shared" information into either region? Previous retrograde tracing studies in the PBN in our lab show that the projection to thalamus is much stronger on the ipsilateral than contralateral side ${ }^{69}$. An analogous study involving the VTA in mice has not been done, but anterograde tracing from the PBN interestingly indicates this projection is stronger contralaterally (Boughter, unpublished data).

\section{Experiment 6.1}

\section{Animals}

36 male and female mice were used in this experiment, including 18 B6 inbred (10 males, 8 females) and 18 KOs (10 males, 8 females). Mice ranged from $62-175$ days old (mean $=96.6$, median $=92)$ at the start of the experiment. Mice of both strains were grouped by i.o. stimulus, including those receiving water ( $\mathrm{n}=6 / \mathrm{strain}), 1 \mathrm{M}$ sucrose ( $\mathrm{n}=6 /$ strain), or $0.003 \mathrm{M} \mathrm{QHCl}(\mathrm{n}=6 /$ strain) on the test day (Table 5-1). Concentrations were chosen on the basis of a previous study from this laboratory which used a similar paradigm ${ }^{59}$.

The experiment tested the hypothesis that FLI in the PBN is elevated specifically in response to taste stimuli by using the trpm 5 -/- mutant mouse model. Transient receptor potential cation channel subfamily M member 5 (TRPM5) KO lines have been generated independently by two separate labs. The KOs in our experiments came from Bob Margolskee's line ${ }^{245}$ as opposed to Charles Zuker's ${ }^{244}$. TRPM5 is a critical cation channel in the transduction of bitter and sweet tastants, and its absence was independently shown to cause a loss of signals in the chorda tympani and IXth ${ }^{244,245}$. Therefore, differences in FLI expression between B6 and KO mice to bitter or sweet tastants can be inferred to be due to disruption of normally taste-responsive neurons.

\section{Immunohistochemistry}

For the nickel-enhanced DAB (tablets, D5905-100TAB; Sigma-Aldrich) staining of c-Fos, sections were rinsed with $0.02 \mathrm{M}$ PBS 3 times. They were then quenched with $3 \%$ hydrogen peroxide $\left(\mathrm{H}_{2} \mathrm{O}_{2}\right)$ for $30 \mathrm{~min}$. After washing in $0.02 \mathrm{M}$ PBS 3 times, sections were incubated overnight ( $\sim 20 \mathrm{~h}$ ) with a rabbit anti-c-Fos primary (sc-52, lot \#F2510; Santa Cruz Biotechnology, CA, USA, diluted to 1:5000; sc-52, lot \#F3016; Santa Cruz Biotechnology, diluted to 1:200; or, ABE457; Millipore, Burlington, MA, USA, diluted to $1: 1000)$ in $0.8 \%$ triton-X 100 in $0.1 \mathrm{M} \mathrm{PB}(0.8 \%$ PBTx) with $5 \%$ normal horse serum (Jackson ImmunoResearch, West Grove, PA, USA) and 0.01\% sodium azide. Following primary incubation, sections were washed 3 times and incubated for $1 \mathrm{~h}$ with donkey anti-rabbit IgG secondary (antibody 111-7103; Rockland, Limerick, PA, USA, diluted to $1: 50$ ) in $0.8 \%$ PBTx. Sections were rinsed 3 times and incubated for $1 \mathrm{~h}$ in $0.2 \%$ peroxidase anti-peroxidase (PAP; Jackson ImmunoResearch, diluted to 1:500) in $0.8 \%$ 
PBTx. DAB solution was made by dissolving DAB tablets in $0.05 \%$ nickel ammonium sulfate in $0.1 \mathrm{M} \mathrm{PB}$ (10 mL for each $10 \mathrm{mg}$ tablet), filtering, and raising the $\mathrm{pH}$ dropwise with $\mathrm{NaOH}$ to 7.4. Sections were rinsed 3 times and reacted in DAB solution for $10 \mathrm{~m}$. The reaction continued for another $10 \mathrm{~m}$ after $\mathrm{H}_{2} \mathrm{O}_{2}$ was added $(14 \mu \mathrm{L}$ to $5 \mathrm{~mL}$ DAB solution; $\sim 0.003 \% \mathrm{H}_{2} \mathrm{O}_{2}$ ) followed by rinsing 3 times in $0.02 \mathrm{M}$ PBS.

\section{Histology}

FLI labeled neurons were plotted and quantified from high-resolution digital microscopic images (150 ppi) taken at 20x magnification using ImageJ (National Institutes of Health, USA) by an observer blind to strain and stimulus. FLI labeling was examined in six sections (every other section) along the rostral-caudal axis from a point about $100 \mu \mathrm{m}$ rostral to the dorsal junction of the scp with the mesencephalic trigeminal tract to the initial appearance of the $\mathrm{m}$, a range of approximately $450 \mu \mathrm{m}$ that corresponded to about -5.25 to $-5.7 \mathrm{~mm}$ from bregma. This span of 6 sections, spaced evenly over $440 \mu \mathrm{m}$, fits approximately within a length $(480 \mu \mathrm{m})$ of the pons corresponding to images 106-110 of the coronal reference atlas at Allen Brain Atlas (which itself corresponds to -5.26 to $-5.66 \mathrm{~mm}$ from bregma). For cell quantification, subnuclei were delineated according to our previous schema ${ }^{50,59,69}$, which in turn was based on the histological delineation of subnuclei in mice by Hashimoto and colleagues (2009) ${ }^{264}$. Based on the aforementioned studies, we are confident this range captures the majority of taste activity in the mouse PBN, which tends to be found at more caudal levels.

The PBN was delineated on both sides of the brain within a section into $\mathrm{dm}, \mathrm{m}$, external medial (em), vl, cl, dl, and el. Moving from rostral to caudal through sections, the last section in which the scp intersected with the mesencephalic tract was identified as $-5.5 \mathrm{~mm}$ post bregma. From this standardized section, the other 5 sections were designated starting at -5.26 and ending at $-5.66 \mathrm{~mm}$ post bregma. Subnuclear delineation was consistent with our previous scheme, derived from cytoarchitectural boundaries evident in cresyl violet-stained sections (Figure 6-1), and based upon delineations amalgamated from several sources ${ }^{50,69,264-266}$. Based on these studies, we are confident this range captures the majority of taste activity in the mouse PBN, which tends to be found at more caudal levels. FLI+ nuclei were only counted as valid if they were at least $50 \%$ within the delineated area, and overlapping $<50 \%$ with another stained nucleus. $75 \%$ of a dark nucleus could be seen and the structure was approximately at $50 \%$ darkness/density relative to others in the section.

\section{Analysis}

After decoding, cell counts were compiled and analyzed. Kolmogorov-Smirnov tests indicated most of the distributions within relevant groups were normal, and the decision to compare FLI between stimuli and strains using parametric ANOVAs (SPSS, v.25) was made, with corrected F-ratios used (Greenhouse-Geisser) where indicated by 

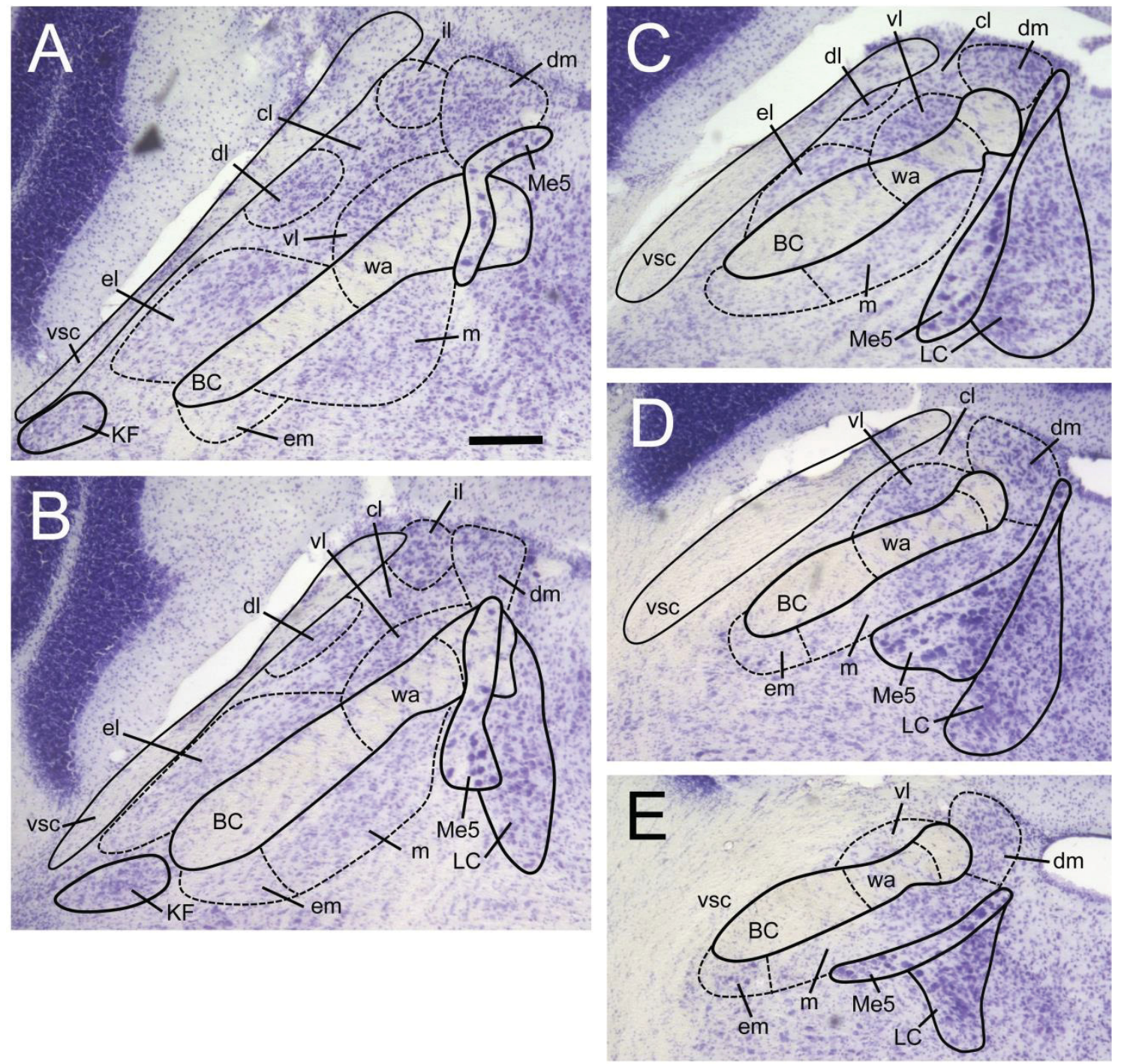

Figure 6-1. Delineation of PBN at 5 rostral-caudal levels.

Sections were Nissl-stained with cresyl violet to reveal cytoarchitecture. This span of sections is spaced out evenly over a $400 \mu \mathrm{m}$ distance, starting at the most rostral section (A) approximately - $5.24 \mathrm{~mm}$ from bregma. Sections are ordered A-E, from most rostral to most caudal. PBN subnuclei are labeled as follows: dm, dorsal medial; m, medial; em, external medial; wa, waist area; vl, ventral lateral; cl, central lateral; dl, dorsal lateral; el, external lateral; il, internal lateral. All pictures at same magnification, scale bar in $\mathrm{A}=$ $100 \mu \mathrm{m}$. vsc, ventral spinocerebellar tract; Me5, mesencephalic trigeminal nucleus; LC, locus coeruleus; BC, brachium conjunctivum; KF, Kölliker-Fuse nucleus. 
Mauchly's test of sphericity. The criterion for significant main effects and interactions was set a priori at $\alpha=0.05$. Where appropriate, post-hoc comparisons were made using Bonferroni's test $(\alpha=0.05)$.

\section{Results 6.1}

Stimuli (sucrose, $\mathrm{QHCl}$, or water [control]) were delivered i.o. to $\mathrm{B} 6$ and $\mathrm{KO}$ mice. Examples of FLI in B6 and KO mice in response to i.o. stimuli are shown in Figure 6-2. Genetic testing confirmed KO mice lacked the trpm 5 gene (Transnetyx, Cordova, TN, USA). In early observations of these sections, we noticed the PBNs of KO mice appeared to have less FLI than B6, which gave us cause to investigate more thoroughly using quantitative analyses.

We did not anticipate finding differences across left and right sides. To test this, data for subnuclei and AP sections were summed and an omnibus ANOVA (withinsubjects factor for side; between-subjects factors for stimulus and strain) was conducted. The data failed to pass Mauchly's test for sphericity and the Greenhouse-Geisser corrected F-ratio was used for determination of side difference. No effect of side was found $\left(\mathrm{F}_{(1,30)}=1.83, \mathrm{p}=0.187\right)$. Further, no interactions were found with stimulus $\left(\mathrm{F}_{(2,30)}\right.$ $=0.66, \mathrm{p}=0.53)$ or strain $\left(\mathrm{F}_{(1,30)}=0.08, \mathrm{p}=0.786\right)$. In between-subjects tests showed a significant effect of strain $\left(\mathrm{F}_{(1,30)}=4.83, \mathrm{p}=0.036\right)$ but not stimulus $\left(\mathrm{F}_{(2,30)}=1.78, \mathrm{p}=\right.$ $0.185)$.

With effects of side ruled out, we averaged the FLI across left and right PBN. We then summed the FLI counts within subnuclei across AP level. Another ANOVA (withinsubjects factor for subnucleus; between-subjects factors for stimulus and strain) was conducted to test the hypotheses that differences would occur between stimulus and strain. Again, the data failed the sphericity test, so Greenhouse-Geisser corrected F-ratios were used. A significant main effect of subnucleus was found $\left(\mathrm{F}_{(6,180)}=35.15, \mathrm{p}<\right.$ $0.001)$, and an interaction was found for subnucleus $\mathrm{X}$ stimulus $\left(\mathrm{F}_{(12,180)}=3.08, \mathrm{p}=\right.$ $0.028)$ but not for subnucleus $X$ strain $\left(\mathrm{F}_{(6,180)}=2.25, \mathrm{p}=0.121\right)$. A between-subjects effect was found for strain $\left(\mathrm{F}_{(1,30)}=4.83, \mathrm{p}=0.036\right)$ but not for stimulus $\left(\mathrm{F}_{(2,30)}=1.78, \mathrm{p}\right.$ $=0.185)$. Therefore, it was strongly evident that FLI was different between strains (Figure 6-3) and the strain effect appeared to occur primarily due to differences in FLI to $\mathrm{QHCl}$ (Figure 6-3C). To test this, we ran ANOVAs on FLI separately for each stimulus (within-subjects factor for subnucleus). Although effects of subnucleus were present in all three tests $(\mathrm{p}<0.001)$, only FLI to $\mathrm{QHCl}$ had significant main $\left(\mathrm{F}_{(1,10)}=5.81, \mathrm{p}=\right.$ $0.037)$ and interaction effects $\left(\mathrm{F}_{(6,60)}=5.13, \mathrm{p}<0.001\right)$. A post hoc Bonferroni test revealed a significant difference in el $(p<0.05)$ but only trends in $\mathrm{dm}(\mathrm{p}=0.166), \mathrm{m}(\mathrm{p}=$ $0.089)$ and $\mathrm{cl}(\mathrm{p}=0.11)$.

We also tested for differences of stimulus using ANOVAs separately for B6 and KO mice (within-subjects factor for subnucleus; Figure 6-4). Again, the effect of subnucleus was significant for both tests $(\mathrm{p}<0.001)$, but neither group had a significant main effect of stimulus $\left(\mathrm{B} 6: \mathrm{F}_{(2,15)}=1.8, \mathrm{p}=0.2 ; \mathrm{KO}: \mathrm{F}_{(2,15)}=2.27, \mathrm{p}=0.138\right)$. 

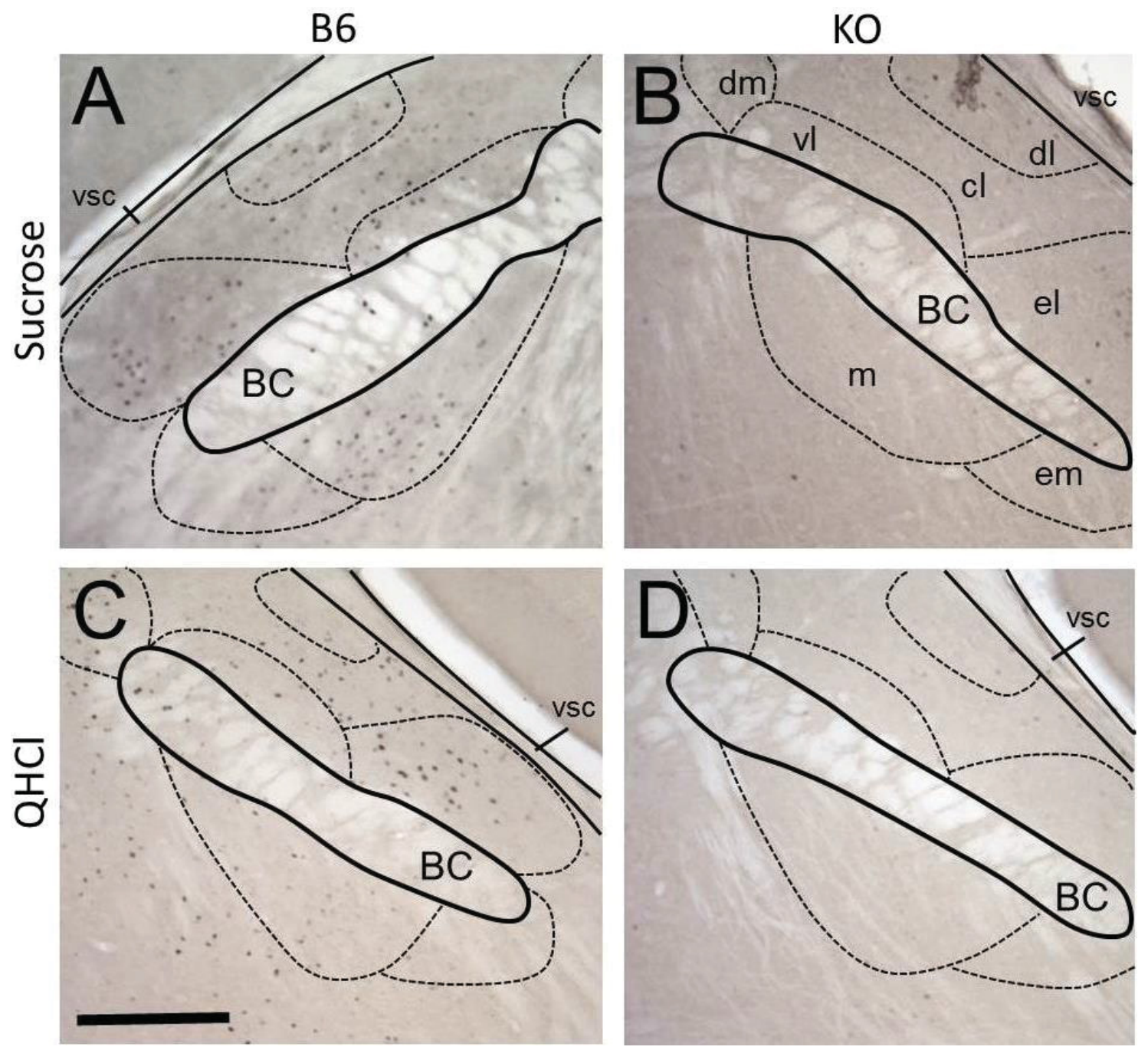

Figure 6-2. FLI in PBN measured with DAB immunohistochemistry in B6 and TRPM5 KO mice.

A,B. Sucrose-elicited FLI. C,D. QHCl -elicited FLI. A, C. FLI in B6 mice. B, D. FLI in TRPM5 KO mice. scp, superior cerebellar peduncle. BC, brachium conjunctivum; dm, dorsal medial; em, external medial; $\mathrm{m}$, medial; vl, ventral lateral; cl, central lateral; dl, dorsal lateral; el, external lateral; vsc, ventral spinocerebellar tract. All pictures at same magnification, scale bar in $\mathrm{C}=100 \mu \mathrm{m}$. 
A
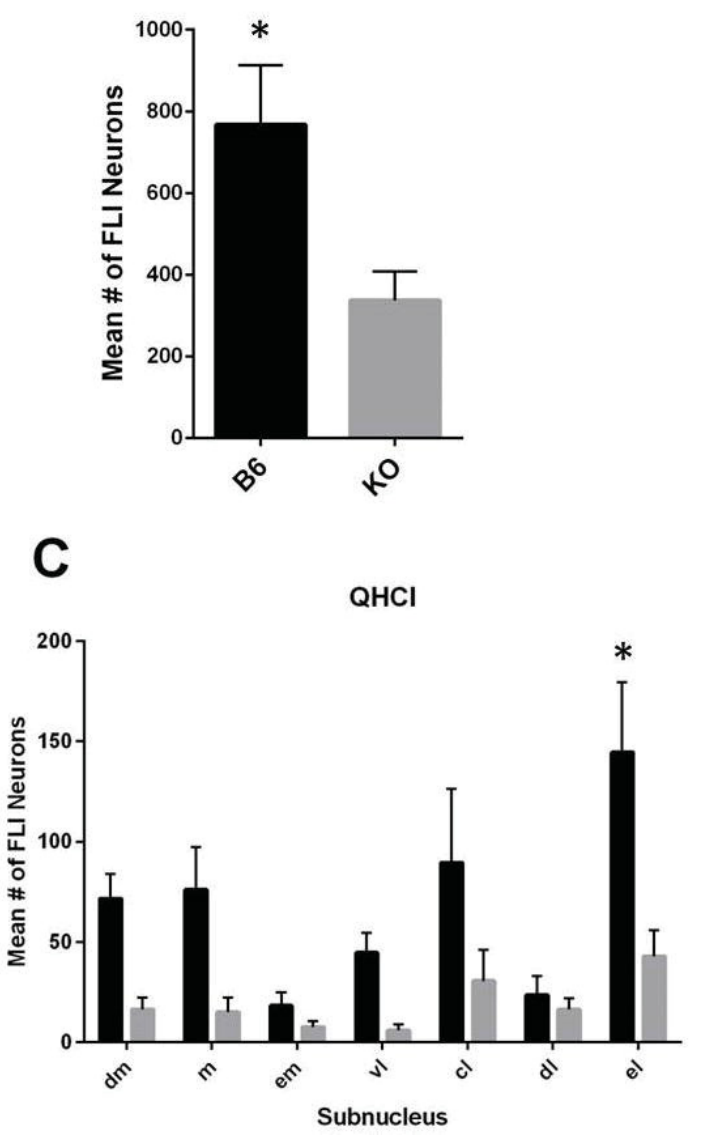
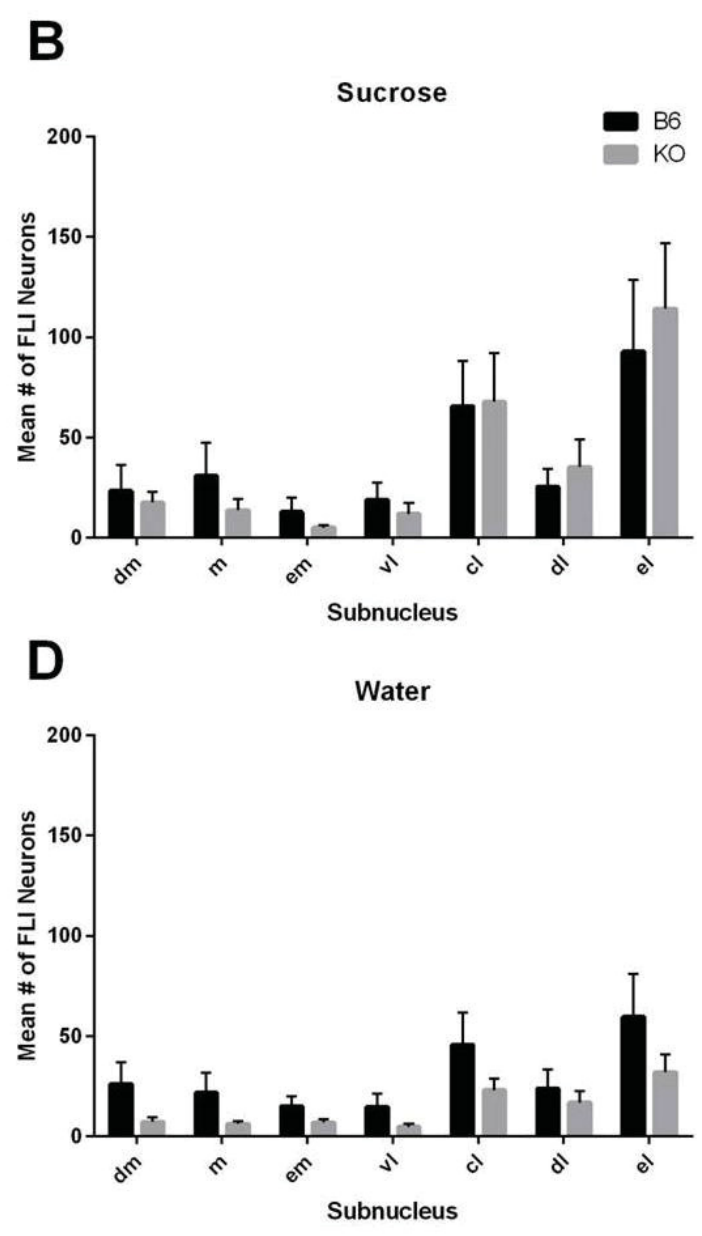

Figure 6-3. FLI in mouse strains and to taste stimuli.

A: Overall, FLI in B6 mice was significantly higher than in KO mice when counts in response to all taste stimuli are combined. This was expected for the mice stimulated with sucrose and $\mathrm{QHCl}$ given that $\mathrm{KO}$ mice are blind to sweet and bitter tastes. B-D: FLI expression in $\mathrm{B} 6$ and $\mathrm{KO}$ mice in each subnucleus, plotted for each intraoral stimulus. FLI expression between the two strains was similar across the PBN for sucrose (B) and water (D). However, B6 mice had significantly higher FLI expression as compared to KO mice in response to $\mathrm{QHCl}$. dm, dorsal medial; m, medial; em, external medial; vl, ventral lateral; cl, central lateral; dl, dorsal lateral; el, external lateral; B6, C57BL/6J mice; KO, trpm5 -/- mice. * $\mathrm{p}<0.05$. 

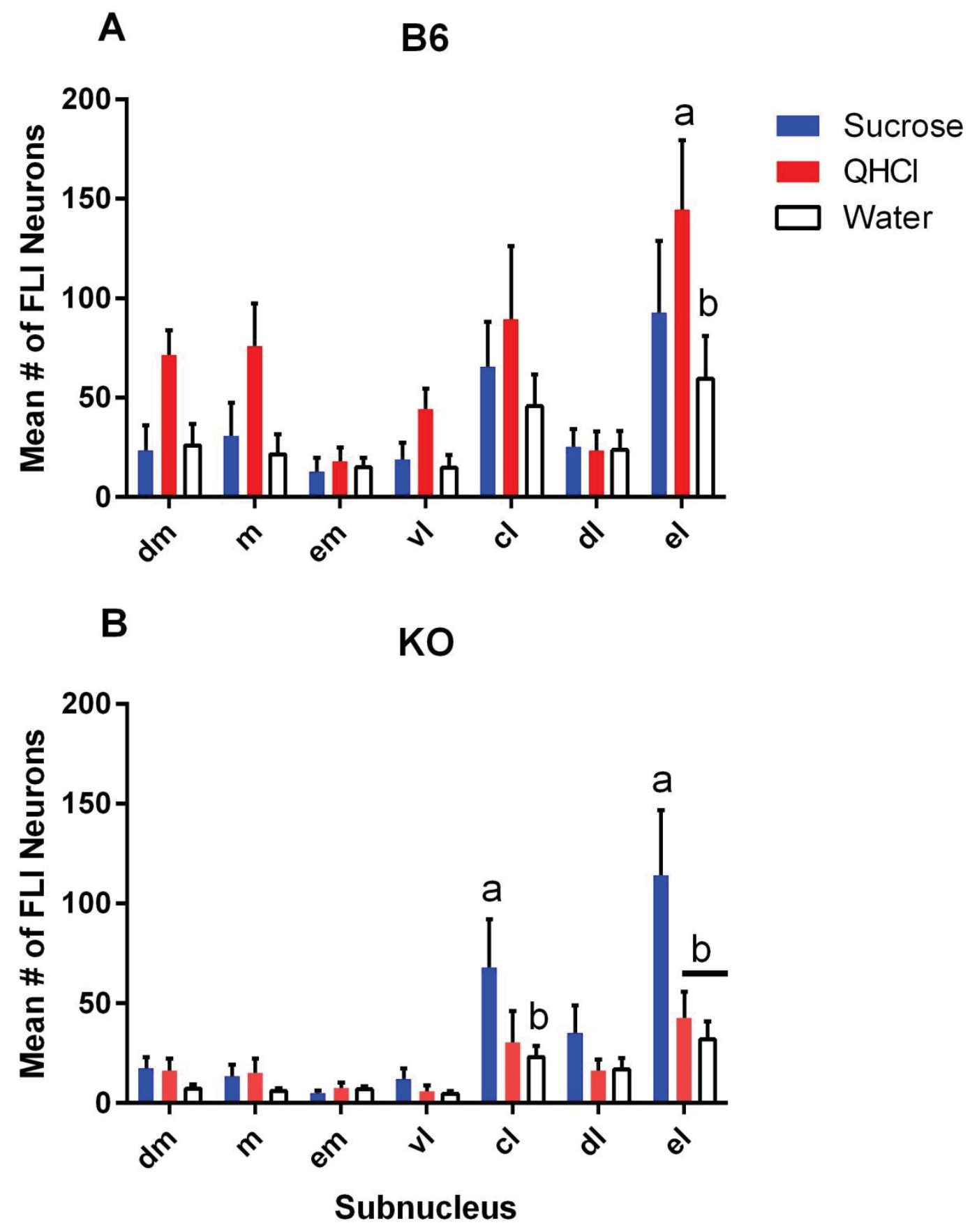

Figure 6-4. FLI in C57BL/6J (B6) and trpm5 -/- (KO) mice in PBN.

Plots of FLI expression evoked by each stimulus across subnuclei for B6 mice (A) and $\mathrm{KO}$ mice (B). These are the same data as shown in Figure 6-3 B-D, but are re-arranged here for a better comparison of the responses to stimuli. Letters denote statistically significant subgroups within particular subnuclei in post-hoc tests $(\mathrm{p}<0.05)$. 
However, we did find a significant interaction of subnucleus and stimulus in $\mathrm{KO}$ mice $\left(\mathrm{F}_{(12,90)}=4.26, \mathrm{p}<0.001\right)$. Post hoc tests in $\mathrm{B} 6$ mice indicated FLI to QHCl in the el $($ mean $=144.6)$ was significantly higher than to water $($ mean $=59.6, p<0.05$; Figure 6-4A). In KO mice, FLI to sucrose in $\mathrm{cl}($ mean $=67.8)$ was significantly higher than to water $($ mean $=23.1, \mathrm{p}<0.05$; Figure 6-4B) and FLI to sucrose $($ mean $=114.2)$ in the el was significantly higher than to $\mathrm{QHCl}($ mean $=42.8, \mathrm{p}<0.05)$ or water $($ mean $=31.9, \mathrm{p}<$ $0.05)$.

\section{Experiment 6.2}

\section{Animals}

14 male and female B6 mice were used in this experiment, ranging from 69 - 126 days in age at the start of the experiment. Mice were grouped by i.o. stimulus, including those receiving water $(n=4), 0.5 \mathrm{M}$ sucrose $(n=6)$ or $0.003 \mathrm{M} \mathrm{QHCl}(\mathrm{n}=4)$ on the test day (Table 5-1). Concentrations were chosen on the basis of a previous study from this laboratory which used a similar paradigm ${ }^{59}$.

\section{Tract Tracing}

Mice were anesthetized and scalped as described in Chapter 3 for i.o. cannula implantation. Mice were mounted in a stereotaxic frame (Stoelting, Wood Dale, IL, USA), and the skull was leveled between bregma and lambda by adjusting the bite bar. On the right side, $1 \%$ cholera toxin subunit $\mathrm{B}(\mathrm{CTb}$; List Biological Laboratories, Campbell, CA, USA) and 5\% Fluoro-Gold (FG; Fluorochrome, LLC, Denver, CO, USA) was microinjected into VPMpc (AP: -1.95, mediolateral [ML]: 0.4, dorsoventral [DV]: $4.3 \mathrm{~mm}$ relative to bregma) and VTA (AP: -3.1 , ML +0.6, DV 4.7), respectively. Tracers were injected by either iontophoresis ( $2 \mathrm{~mA}, 5 \mathrm{~s}$ on/off for $15 \mathrm{~min}$ ) with a precision constant current source (CAT. NO. 51595; Stoelting, Wood Dale, IL, USA) or pressure with a picospritzer (52-302-900; General Valve Corporation, Fairfield, NJ, USA). Glass micropipettes ( $30-35 \mu \mathrm{m}$ tip diameter for iontophoresis; $50-60 \mu \mathrm{m}$ for pressure) were positioned with a micromanipulator (SM-191; Narishige, Tokyo, Japan) and left unaltered for $10 \mathrm{~min}$ prior and after injections. I.o. cannulas were implanted on the ipsilateral side of the tracer microinjections. Animals were allowed to recover for 1 week following surgery, during which time i.o. cannulas were periodically flushed with water. Following this period, they were trained for 3 consecutive sessions with water administration through the cannula, then tested on the 4th day with solutions as described in the general methods, followed by perfusion. 


\section{Immunohistochemistry}

We stained the sections as in Chapter 3. Prior to the primary incubation, we used $3 \%$ normal donkey serum for blocking (Jackson ImmunoResearch). Then, we incubated sections in goat anti-CTb (List Biological, diluted to 1:5000) and rabbit anti-c-Fos (sc-52, lot \#F2510; Santa Cruz Biotechnology, diluted to 1:5000; sc-52, lot \#F3016; Santa Cruz Biotechnology, diluted to 1:500; or, ABE457, Millipore, diluted to 1:2000). Next, we used biotinylated donkey anti-goat IgG for secondary (Jackson ImmunoResearch, diluted to 1:1000) and the fluorescent cyanine dye, Cy3-conjugated streptavidin for a fluorophore (Jackson ImmunoResearch, diluted to 1:1000). In the last steps, biotinylated goat antirabbit for secondary (Jackson ImmunoResearch Laboratories, West Grove, PA, USA), and streptavidin-Cy2 (Jackson ImmunoResearch Laboratories, West Grove, PA, USA) or streptavidin-Alexa Fluor 568 (Molecular Probes/Invitrogen, San Diego, CA, USA) diluted to a concentration of 1:1000. Several sections were placed in the same solution only lacking the primary antibody to serve as a negative control. No FLI was observed in control sections.

\section{Quantification and Analysis}

Cell plots and counts were made from high-resolution digital images (150 ppi) taken at 20x magnification of 3-color fluorescent labeling, using ImageJ by an observer blind to stimulus group. The range of sections and subnuclear delineation was similar to Experiment 6.1. PBN labeling of tracers and FLI was examined in 5 sequential sections (every other section) along the rostral-caudal axis. This span of sections, spaced evenly over $360 \mu \mathrm{m}$, fits approximately within a length $(400 \mu \mathrm{m})$ of the pons corresponding to images 106-110 of the coronal reference atlas at Allen Brain Atlas. The PBN was delineated on both sides of the brain within a section into dm, m, em, vl, cl, dl, and el, and here the "waist" region (i.e., within the brachium wa) and internal lateral (il) were added.

Each type of label (FG, CTb, and FLI) was initially counted from a single channel color image. Only complete cellular (FG, CTb) or nuclear (FLI) profiles were counted. Moreover, intensity of the profile had to exceed the average background intensity, which was determined for each image from a 150 X 150 pixel ROI placed over a non-PBN region of the brainstem within that image. Double-labeled (DL) and triple-labeled cells were then counted using merged images. Data was assessed for normality, which it passed. To assess whether appetitive or aversive taste stimuli preferentially elevated activity in subnuclei or pathways, we analyzed data collected by projection, FLI positivity, and double labeling. ANOVAs were used to test for differences, followed by post-hoc tests where appropriate, and significance level was $\alpha=0.05$. 


\section{Results 6.2}

\section{Tracing}

Tracer injection sites in both the VTA and VPMpc were reconstructed for all mice and are shown in Figure 6-5. An example of fluorescent labeling, taken at 10X magnification to show the entire PBN, is shown in Figure 6-6. In this example, taken from the caudal PBN (similar to Figure 6-1D), FG expression is stronger on the contralateral side; cells labeled with this tracer tend to be located dorsally within the nucleus. CTb expression, on the other hand, was greater on the ipsilateral side, with the strongest expression medially and in the wa. FLI expression (evoked by i.o. sucrose in this mouse) is roughly similar within the PBN on both sides, and is also found expressed medial to the PBN itself, in the mesencephalic trigeminal nucleus (Me5) and locus coeruleus (LC). More detailed examples of tracer and i.o.-evoked FLI labeling, and DL cells, are shown in Figure 6-7.

Initially, we examined overall counts of labeled cells for each tracer, and for FLI, in each stimulus group and on both sides of the brain. Based on previous tracing studies, we expected that the 2 retrograde tracers (injected into mice several weeks before i.o. stimulation) would differ in expression on either side of the brain, and across PBN subnuclei, but would not differ according to stimulus group. This hypothesis was tested with a 3-way ANOVA for each tracer (within-group factors for side and subnucleus, and a between-group factor for stimulus). For FG expression, there were significant effects of side $\left(\mathrm{F}_{(1,11)}=23.15, \mathrm{p}=0.001\right)$ and subnucleus $\left(\mathrm{F}_{(8,88)}=49.73, \mathrm{p}<0.001\right)$, but not stimulus. Only the side $\mathrm{X}$ subnucleus interaction was significant $\left(\mathrm{F}_{(8,88)}=11.72, \mathrm{p}<\right.$ 0.001). Total FG expression (collapsed across group and subnucleus) was lower on the ipsilateral (mean total cells/animal $=284.86$ ) side as compared to the contralateral (mean cells/animal $=421.64)$ side. In general, FG expression was highest in the $\mathrm{dm}, \mathrm{m}, \mathrm{vl}$ and $\mathrm{cl}$ subnuclei relative to other subnuclei. Comparing individual subnuclei across side (i.e., collapsed across group) showed significant increases of labeled cells in the dm, $\mathrm{m}$, and vl subnuclei on the contralateral side.

For $\mathrm{CTb}$, the 3-way ANOVA similarly yielded significant effects of side $\left(\mathrm{F}_{(1,11)}=\right.$ $73.24, \mathrm{p}<0.001)$ and subnucleus $\left(\mathrm{F}_{(8,88)}=66.90, \mathrm{p}<0.001\right)$, as well as a significant side $\mathrm{X}$ subnucleus interaction $\left(\mathrm{F}_{(8,88)}=54.29, \mathrm{p}<0.001\right)$. However, stimulus did not significantly impact counts. Unlike FG, CTb was expressed in overall greater numbers on the ipsilateral side (mean counts $=578.36$ vs. 202.21 for ipsilateral and contralateral sides, respectively). CTb also varied from FG in its subnuclear expression, notably in its robust expression in the el, reflecting a difference in topography of neurons projecting to these distinct brain regions. When the sides were statistically compared (collapsed across group), larger numbers of labeled cells were found ipsilateral in the m, wa, and el subnuclei. The fact that only a handful of cells were found to collateralize to both VTA and VPMpc (3-7 per mouse, either side), further indicates the independence of these projections. 

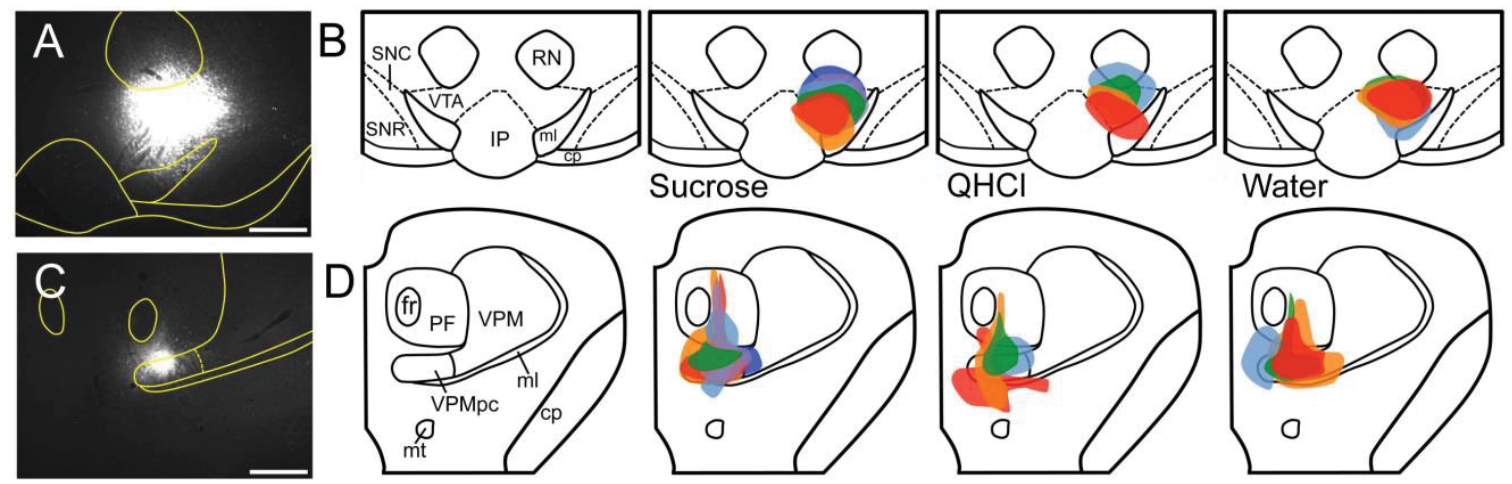

Figure 6-5. VTA and VPMpc injection sites.

A. Representative fluorescent image of FG injection site in the VTA. B. Plots of injection site and size in the VTA for each animal according to stimulus group. C. Representative fluorescent image of CTb injection site in the VPMpc. D. Plots of injection site and size in the VPMpc for each animal according to stimulus group. Colors identify single animals within each group, consistent for injections at both brain sites. Scale bars $=1$ $\mathrm{mm}$. SNC, substantia nigra pars compacta; SNR, substantia nigra, pars reticulata; IP, interpeduncular area; ml, medial lemniscus; cp, cerebral peduncle; RN, red nucleus; fr, fasciculus retroflexus; PF, parafascicular thalamic nucleus; VPM, venteroposteromedial nucleus of thalamus; $\mathrm{mt}$, mammillothalamic tract.
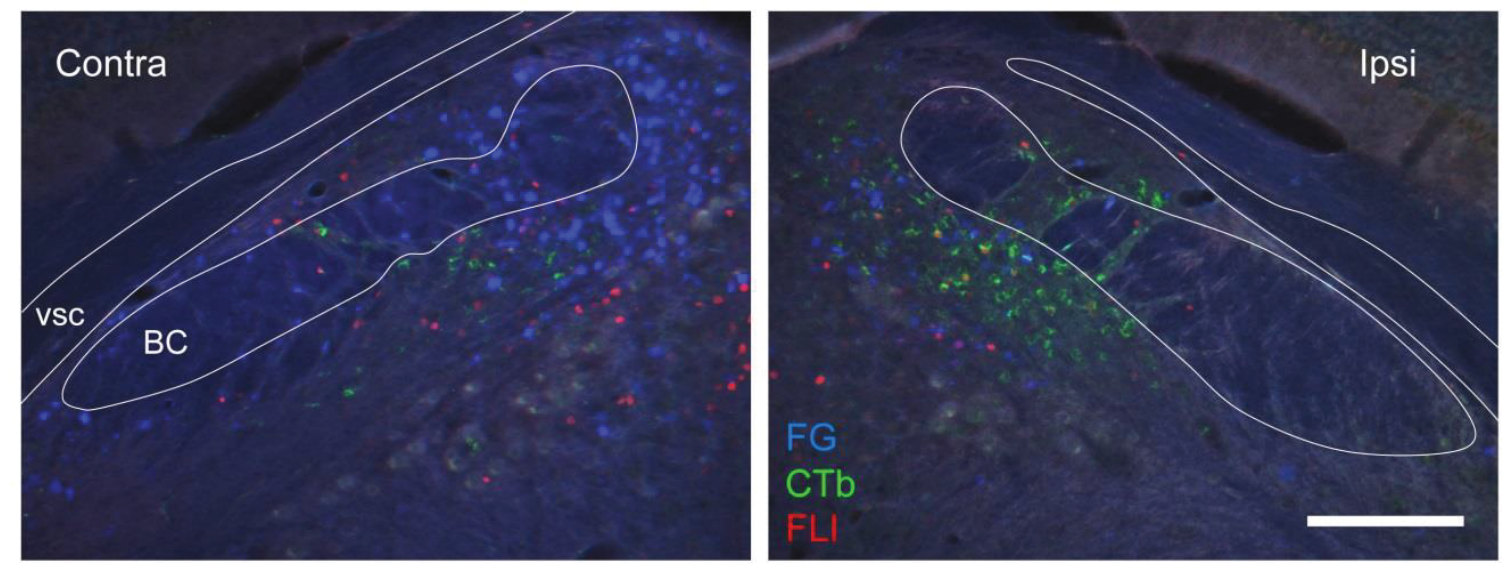

\section{Figure 6-6. Labeling in the caudal PBN.}

Low-power (10X) fluorescent images taken from both sides of a single brain section; the mouse received sucrose stimulation. Retrograde FG labeling (blue) from the VTA was stronger on the contralateral side, whereas retrograde $\mathrm{CTb}$ labeling (green) was stronger on the ipsilateral side. Expression of sucrose-evoked FLI (red) was similar on both sides. Scale $\mathrm{bar}=200 \mu \mathrm{m}$. 

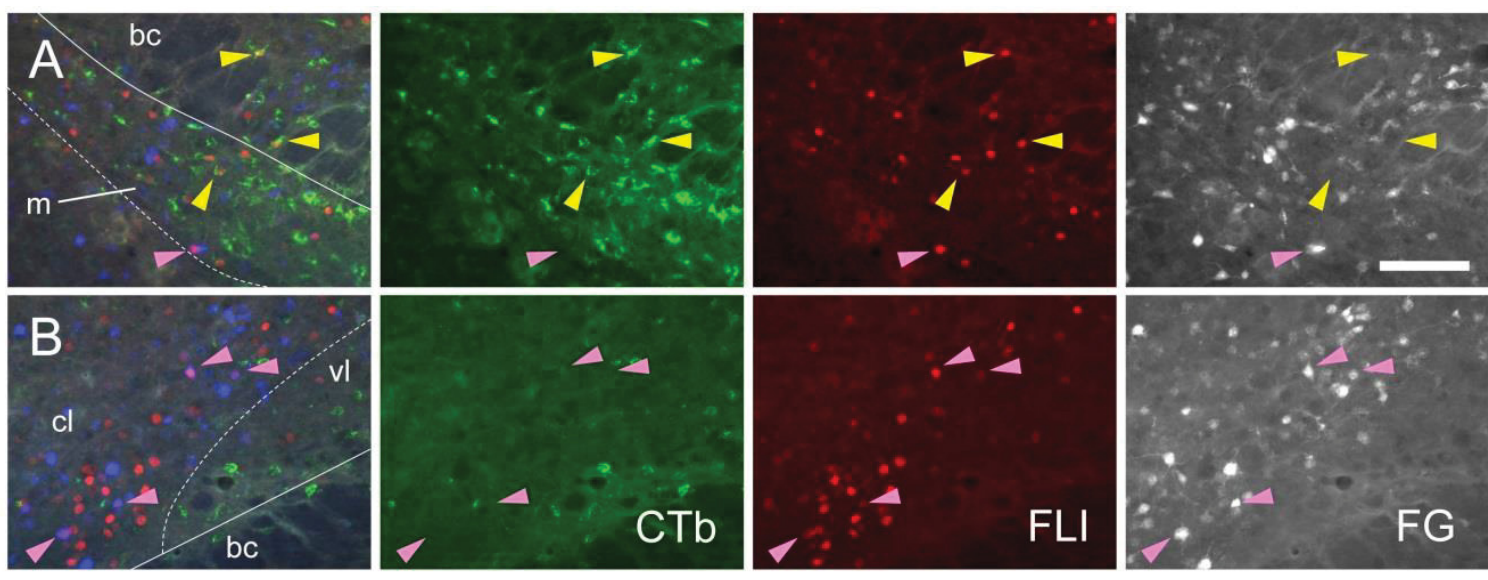

Figure 6-7. Examples of FG, CTb, and FLI labeling in the PBN.

Images shown merged, as well as individual channels for each fluorophore (FG, blue in the merged image, is shown more clearly in greyscale). The stimulus for each set of images (from separate mice) was sucrose. A. Several cells doubled-labeled for CTb and FLI (yellow arrowheads) are found in the ipsilateral, caudal PBN in the medial (m) subnucleus. B. Cells double-labeled for FG and FLI (pink arrowheads) in the central lateral $(\mathrm{cl})$ subnucleus on the contralateral side in a more rostral level of the PBN. All images same magnification, scale bar $=100 \mu \mathrm{m}$. 


\section{FLI and Double-labeled Cells}

Because FLI did not significantly differ on either side of the brain, counts were averaged across sides and analyzed according to i.o. stimulus (Figure 6-8; 2-way ANOVA, subnucleus $\mathrm{X}$ stimulus). There was a significant effect of both subnucleus $\left(\mathrm{F}_{(8}\right.$, $\left.{ }_{88)}=23.83, \mathrm{p}<0.001\right)$ and stimulus $\left(\mathrm{F}_{(2,11)}=13.82, \mathrm{p}=0.001\right)$, as well as a significant interaction $\left(\mathrm{F}_{(16,88)}=6.34, \mathrm{p}<0.001\right)$. Post-hoc multiple comparison tests $(\mathrm{p}<0.05)$ between stimuli at each subnucleus indicated significant differences in 5/9 comparisons. Each stimulus resulted in different counts in the dm, where QHCl evoked the most FLI, and water the least. Sucrose and QHCl both evoked more FLI than water in the $\mathrm{m}$ and el, but did not differ from each other. Sucrose stimulation resulted in more FLI than either $\mathrm{QHCl}$ or water in the dl, and sucrose, but not $\mathrm{QHCl}$, evoked more FLI in the cl.

Cells DL for both tracers, or one tracer and FLI, were generally found in low amounts $(<7 \%$ for all types) relative to overall counts of each marker type (only a couple of cells were triple-labeled across all mice and are not dealt with any further). DL cells were therefore examined as total counts across all subnuclei. The number of cells doubled-labeled for either FG + FLI, or CTb + FLI, likely depends on the total numbers of cells expressing each individual marker. When expressed as a percentage of tracermarked cells, there was a significant effect of stimulus group $\left(F_{(2,22)}=7.88 ; p=0.003\right)$, but not side, on the percentage of cells doubled-labeled for FG and FLI, with higher numbers of these in sucrose-stimulated mice on the ipsilateral side relative to water, but not $\mathrm{QHCl}$. There were significant effects of both stimulus $\left(\mathrm{F}_{(2,22)}=9.79 ; \mathrm{p}<0.001\right)$ and side $\left(\mathrm{F}_{(1,22)}=5.97 ; \mathrm{p}=0.023\right)$ on the percentage of cells doubled-labeled for $\mathrm{CTb}$ and FLI. Significantly more DL cells were found in the sucrose and $\mathrm{QHCl}$ groups relative to water on the ipsilateral side. However, when expressed as a percentage of total FLI expression in each mouse, no significant differences among stimulation groups occurred for either FG + FLI or CTb + FLI cells. Collectively, this evidence suggests that the double-label measure (i.e., co-expression of FLI and tracers) is most likely proportional to the amount of FLI evoked by the i.o. stimulus. Although these i.o. stimuli consistently evoked activity in a small proportion of tracer-labeled cells, the hypothesis that stimuli of different qualities differentially elevate activity in neurons projecting to either VTA or VPMpc is not supported.

\section{Conclusions}

In Experiment 6.1, B6 mice had higher levels of expression of FLI than TRPM5 $\mathrm{KO}$ mice in the PBN following i.o. administration of taste stimuli. On the surface, this result was expected based on behavioral and electrophysiological studies with these KO mice that show an attenuation, or even total lack of, oral sensitivity to sweet and bitter tastants ${ }^{244,245}$. However, a careful examination of FLI expression in each strain across subnuclei suggested that this effect depended on which stimulus was used. In particular, the strain difference appeared to largely rely on the effects of increased FLI to QHCl in B6 mice. Intriguingly, sucrose-evoked FLI was basically similar between strains. It is possible that sucrose evokes taste or orosensory responses in $\mathrm{KO}$ mice through a 


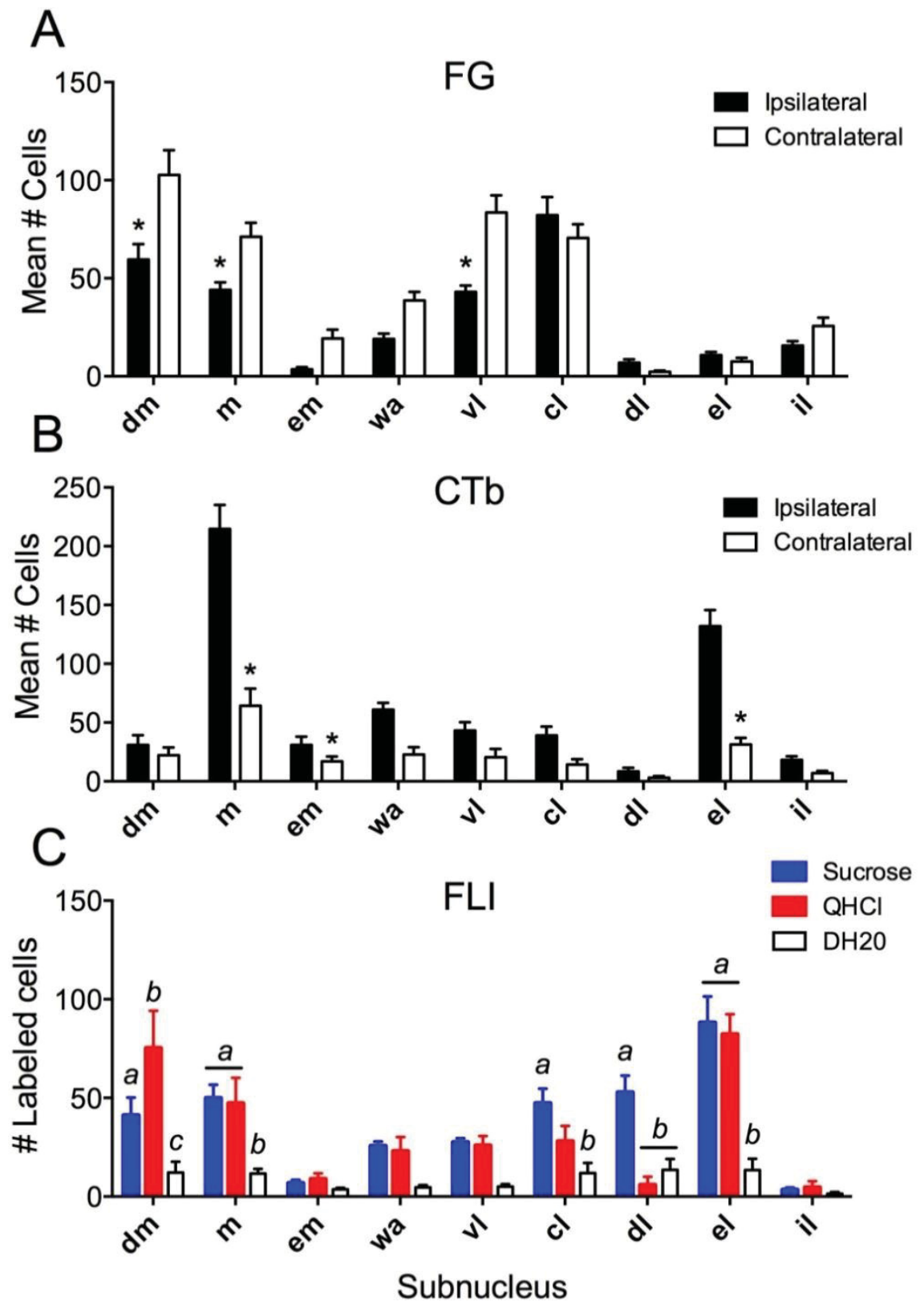

Figure 6-8. Quantification of FG, CTb, and FLI labeling in the PBN by subnucleus.

A. Mean number of VTA-projecting ( $\mathrm{FG}^{+}$) neurons, combined across stimulus groups, varied according to both subnuclear location and side of the brain. B. Mean number of VPMpc-projecting $(\mathrm{CTb}+)$ neurons, combined across stimulus groups, also varied according to subnucleus and side. C. Mean FLI expression by intraoral stimulus group, plotted for each subnucleus-here data from both sides of the brain are averaged for each animal. Asterisks in A, B show significant ipsilateral-contralateral differences in particular subnuclei $(\mathrm{p}<0.05)$. Letters/horizontal bars denote statistically different groups $(\mathrm{p}<0.05)$ in $\mathrm{C}$. 
TRPM5-independent pathway. The effects of TRPM5 are thought to be specific to sweet receptor (T1R) and bitter receptor (T2R) expressing taste cells. In the transduction of these classes of stimuli, TRPM5 plays a vital role in taste cells as a calcium-gated cation channel; when this channel opens in response to elevated intracellular calcium it produces a depolarizing generator potential ${ }^{2}$. However, recent studies uncovered a host of nonT1R mechanisms expressed in taste cells that may play a role in sweet transduction, including sugar transporters, metabolic sensor channels, and brush-border enzymes ${ }^{267,268}$. Though, an important caveat to this possibility is that although alternative pathways for sweet transduction exist, mice without TRPM5 show severely diminished behavioral sensitivity to sucrose ${ }^{245,246}$, yet in our study sucrose was equally potent as an activator of FLI in KO mice as it was in B6 mice (Figure 6-3B).

A more likely explanation is that the robust FLI in response to sucrose in both strains stems from viscerosensory effects. Even a diminished preference to the sucrose in the $\mathrm{KO}$ mice might lead to them consuming the solution during i.o. stimulation in a manner similar to the B6 mice. Further, the mild restriction both strains underwent before testing might also explain consumption of a stimulus by $\mathrm{KO}$ mice. $\mathrm{B} 6$ mice likely consumed more sucrose and less $\mathrm{QHCl}$ because of perceived palatability, and $\mathrm{KO}$ mice likely consumed all three stimuli at the same rate that B6 consumed water. Importantly, PBN effects may not strongly depend on volume consumed. Therefore, the elevated FLI could represent some aspect of consummatory behavior, such as gastric load, nutrient signaling in the small intestine, or feedback from other brain areas concerned with the onset of satiety. Indeed, the most robust response to sucrose occurred in the lateral PBN, in the $\mathrm{cl}$ or el, areas known to have a definite visceral-related function. Interestingly, $\mathrm{QHCl}$ evoked a much stronger response in $\mathrm{B} 6$ mice than in $\mathrm{KO}$ mice in some of these same lateral subnuclei. In previous experiments we have conducted in B6 mice using i.o. stimulation with $\mathrm{QHCl}$ (Boughter, unpublished), it is clear that the mice do not consume much of this stimulus; instead they display an obvious aversive reaction, even letting much of the solution drain from their mouths as they shake their heads. Therefore, elevated FLI in the B6 mouse (and the lack of it in the $\mathrm{KO}$ ) in response to QHCl surely represents a taste- or at least orosensory-mediated effect. This in turn suggests that these lateral PBN subnuclei, especially $\mathrm{cl}$ and el, contain both taste- and viscerally-related neurons. This idea has much support in the literature ${ }^{73}$. In fact, in rats, it has been noted that aversive stimuli such as QHCl or hydrochloric acid produce FLI in an "inner" division of the el, whereas visceral stimulation occurs in primarily an "outer" layer of this same subnucleus ${ }^{66,269}$. In the much smaller el subnucleus in mice, any cytoarchitectural or functional division remains uncertain; in the present study, it is not clear that sucrose and QHCl FLI are topographically distinct. Future studies examining the independence of these modalities in the PBN could use next generation techniques such as TRAP to visualize activity to multiple stimuli, or to specifically interrogate the function of responsive neurons (e.g. McCullough et al., $2016^{270}$ ).

The results of Experiment 6.1 impact the interpretation of the results of Experiment 6.2, in that stimulus-evoked FLI of projection neurons can be categorized by either their taste or visceral responsiveness. In this experiment, we found that sucrose and $\mathrm{QHCl}$ were roughly equivalent in terms of their FLI in either VTA or VPMpc-projecting 
neurons. This equivalence extended to water, when the total amount of FLI expression was considered. The projection to VTA was stronger on the contralateral side, and almost completely independent of the projection to VPMpc (few cells DL for these tracers). This contrasts with prior findings that single PBN neurons often collateralized to two forebrain regions, i.e. VPMpc and AMY, or VPMpc and $\mathrm{LH}^{69}$. It is also interesting that VTAprojecting neurons were only rarely found in the el subnucleus, an area (as discussed above) with robust bitter taste and visceral responses. Of course, physiology studies find bitter-responsive neurons in other subnuclei as well ${ }^{49}$, and tracing studies indicated a mix of taste (rNST) and visceral (cNST) to most parts of $\mathrm{PBN}^{76}$. Therefore, activation of VTA projection neurons by sucrose or $\mathrm{QHCl}$ in other $\mathrm{PBN}$ regions may still be visceral or orosensory in nature, respectively.

We did notice a difference between stimulus-evoked FLI in the two experiments, with a significant effect of stimulus present in Experiment 6.2 but not in 6.1. The main driver in this difference was a greater level of water-evoked FLI in Experiment 6.1. It is possible that the different immunohistochemical techniques used for FLI in both experiments contributed to this difference, with a greater level of sensitivity perhaps found with the DAB staining technique as opposed to immunofluorescence. 


\section{CHAPTER 7. LOCATION AND MORPHOLOGY OF VTA NEURONS RESPONSIVE TO TASTE STIMULI WITH VARIED VALENCE}

NAc DA release and VTA activity in response to palatable taste stimuli has been well-established. It was previously found that the VTA increased FLI in response to palatable meals ${ }^{212}$. First, we wanted to replicate this result and see if it was primarily a taste-evoked response or dependent on post-ingestional feedback. To achieve this end, we used TRPM5 KO mice as was mentioned in Chapter 6. Given that these mice were shown to be taste-blind to sweet and bitter stimuli ${ }^{244,245}$, they allowed us to separate out responses to post-ingestional input. A second experiment set out to determine if activity in known VTA cell types produced a differential pattern based on taste valence.

We wanted to see if bitter and aversive QHCl would elicit FLI in the VTA as might be inferred from studies that showed the VTA to be responsive to aversive footshock ${ }^{80}$ and the NAc to release DA to various aversive stimuli ${ }^{127}$. Although the VTA responds to both palatable and aversive tastes, its DA neurons generally increase their phasic responses to palatable stimuli and decrease to aversive, although a small percentage are actually excited by aversive air puffs ${ }^{92,151}$. Further investigation may help to explain some of the more nuanced responsiveness of the VTA. Both of the experiments in this chapter tested for responses to bitter $\mathrm{QHCl}$ and compared them to those to sweet sucrose.

The VTA may differentiate palatable and aversive tastes using separate anatomical pathways and with distinct activity within specific cell types or projections. Several subnuclei have long been defined within the VTA based on clustering patterns of $\mathrm{TH}+$ cells ${ }^{156}$ and projection ${ }^{94}$. Although the VTA is known for being primarily DAergic, a relatively high population of GABA cells was also previously found ${ }^{201}$. Some GABA cells in the VTA project to the same forebrain areas as the DAergic ${ }^{198}$, and VTA DA and GABA neurons have been classified into various types based on responses to conditioned and reward stimuli ${ }^{92}$. It would be interesting to know how activity in each of these cell populations in the VTA compares based on stimulation with pleasurable and displeasurable stimuli.

Additionally, we included the RRF and RMTg in our measurements of the midbrain anatomy. The RRF is a separate but related nucleus to the VTA; it has been anatomically well-studied but remains functionally nebulous ${ }^{271,272}$. Waraczynski and Perkins (2000) ${ }^{272}$ showed that inactivating it causes a loss in the reinforcing effect of electrically stimulating the medial forebrain bundle. Thus, the RRF was included in this study to determine if it contributed to taste reward. One longstanding controversy regarding the VTA GABA cells and subnuclei concerns whether the RMTg should be considered a VTA subnucleus. While we did not set out to resolve the issue, we did use our possession of transgenic mice with green fluorescent protein (GFP) hemizygously expressed on their gadl promoters (GAD67-GFP mice) to attempt to observe this lesser understood subnucleus. Previous studies located the subnucleus in the posterior end of the VTA, sometimes referring to it as the "tail of the VTA" 166,194,197. We used these 
studies along with our other delineation methods to attempt to quantify GAD67 neurons in the RMTg as well as the rest of the VTA.

To evaluate VTA activity with respect to particular regional and cell type populations, we conducted two experiments in which labeled neurons responsive to taste stimuli were quantified. Experiment 7.1 was a continuation of Experiment 6.1, but instead taste-evoked FLI was examined in the VTA. A second experiment used fluorescent labeling and confocal microscopy to observe FLI in DA or GABA cells in the VTA's subnuclei and the RRF. The overall goal was to discover patterns in VTA activity indicating how it encodes appetitive and aversive tastes.

\section{Experiment 7.1}

The same 36 B6 and KO mice used to collect DAB-stained FLI data from the PBN in Experiment 6.1 were also used for the VTA. Likewise, the same nickel-enhanced DAB immunohistochemistry was used to measure FLI in the VTA (Figure 7-1).

As an anatomical reference to align the section's AP level, moving from rostral to caudal sections, the first section in which the medial lemniscus (ml) touched the cerebral peduncle (cp) was identified as $-3.64 \mathrm{~mm}$ (post) bregma. FLI was measured separately in 3 sections used in previous experiments to approximate the aVTA $(-3.08)$, pVTA (-3.52), and tVTA $(-3.88)^{67,160-164,166,167,262}$. Except that counts were being done in the VTA, the same quantitative conditions for validity of FLI-positivity were applied as those done in Experiment 6.1 for the PBN. FLI labeled neurons were plotted and quantified from highresolution digital microscopic images (150 ppi) taken at 20x magnification using ImageJ (National Institutes of Health, USA) by an observer blind to condition.

After counting, conditions were revealed so the data could be categorized by strain and stimulus. Kolmogorov-Smirnov tests indicated most of the distributions were normal, and FLI was compared using parametric ANOVAs (SPSS). Greenhouse-Geisser corrected F ratios were used where Mauchly's test indicated the sphericity assumption was not met. Significance level was $\alpha=0.05$ for main effects and interactions, and post hoc Bonferroni tests were used where appropriate $(\alpha=0.05)$.

\section{Results 7.1}

Fos was stained in the VTA with DAB to measure FLI in B6 and KO mice after i.o. stimulation with palatable sucrose, aversive $\mathrm{QHCl}$, and neutral water. In initial observations of these sections (Figure 7-1), those of B6 appeared to have higher FLI than sections taken from $\mathrm{KO}$ mice. Thus, we tested this quantitatively.

We had no reason to expect left-to-right side differences, so we summed FLI in the AP sections and conducted an omnibus ANOVA (within-subjects factor for side; between-subjects factors for stimulus and strain). The data failed to pass Mauchly's test 

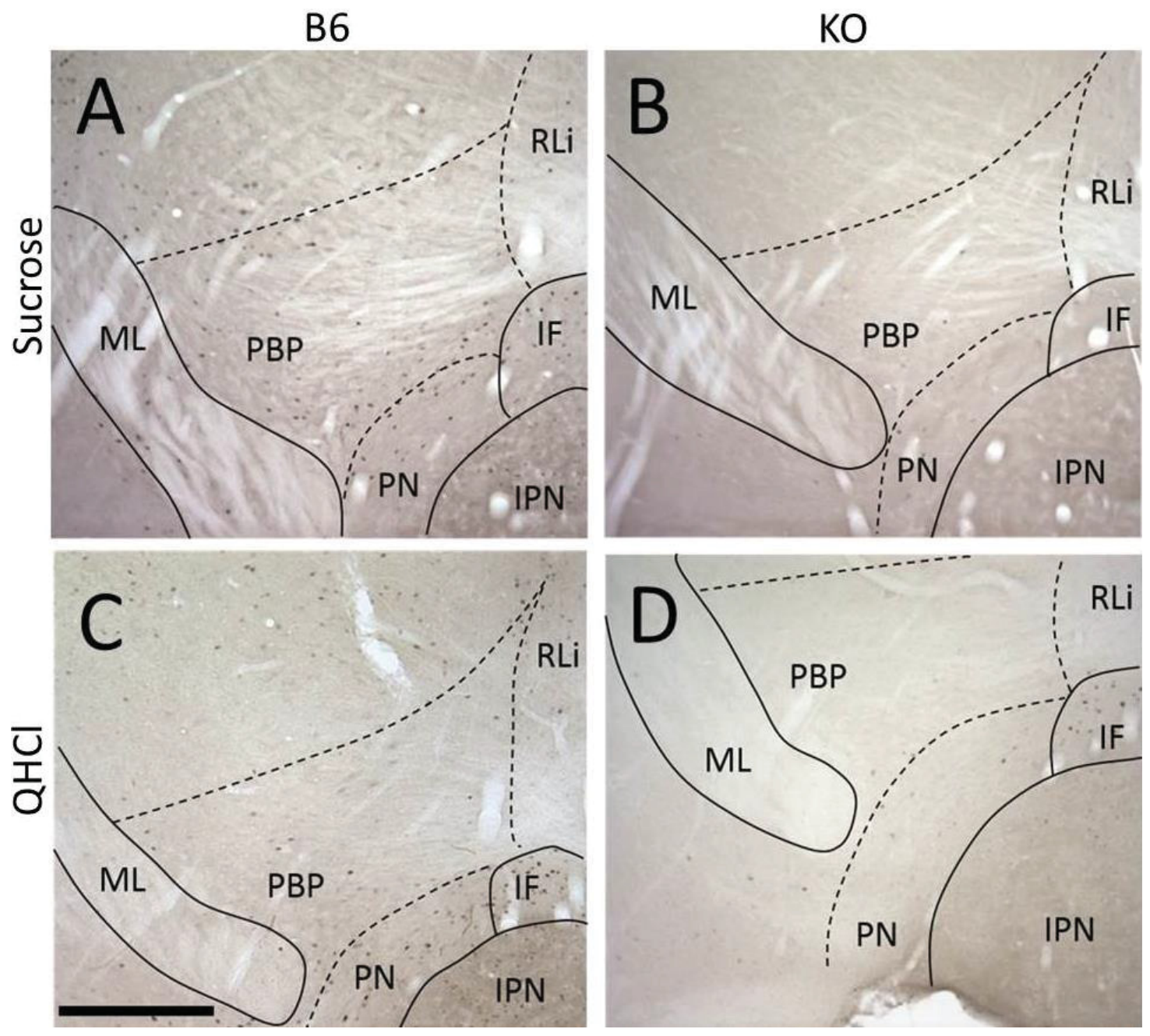

Figure 7-1. FLI in VTA measured with DAB immunohistochemistry in B6 and TRPM5 KO mice.

A, B. Sucrose elicited FLI. C, D. QHCl-elicited FLI. A, C. FLI in B6 mice. B, D. FLI in TRPM5 KO mice. IF, interfascicular nucleus; IPN, interpeduncular nucleus; ML, medial lemniscus; PBP, pigmented parabrachial nucleus; PN, paranigral nucleus; RLi, raphe linear nucleus. Scale bar $=100 \mu \mathrm{m}$. 
for sphericity and the Greenhouse-Geisser corrected F-ratio was used for determination of side difference. No effect of side was found $\left(\mathrm{F}_{(1,30)}=0.88, \mathrm{p}=0.357\right)$. Further, no interactions were found of side with stimulus $\left(\mathrm{F}_{(2,30)}=0.21, \mathrm{p}=0.81\right)$ or $\operatorname{strain}\left(\mathrm{F}_{(1,30)}=\right.$ $0.13, \mathrm{p}=0.725)$. Between-subjects tests were not significant for strain $\left(\mathrm{F}_{(1,30)}=1.27, \mathrm{p}=\right.$ $0.268)$ or stimulus $\left(\mathrm{F}_{(2,30)}=0.95, \mathrm{p}=0.399\right)$.

With effects of side ruled out, we averaged the FLI across left and right VTA. Since prior studies suggested differences in AP levels of VTA ${ }^{164}$, our follow-up ANOVA expanded this as a within-subjects factor (and kept between-subjects factors for stimulus and strain). We expected to find a significant strain difference reflecting our visual observation of FLI (Figure 7-1); stimulus group differences were also expected, especially for sucrose, which is palatable (and rewarding), compared to neutral water. AP level data passed Mauchly's test of sphericity. The effect of AP level was significant $\left(\mathrm{F}_{(2}\right.$, $\left.{ }_{60)}=8.62, \mathrm{p}=0.001\right)$; however, no effects of strain $\left(\mathrm{F}_{(1,30)}=1.27, \mathrm{p}=0.268\right)$ or stimulus $\left(\mathrm{F}_{(2,30)}=0.95, \mathrm{p}=0.399\right)$ were found. Neither were interactions for AP level with strain $\left(\mathrm{F}_{(2,60)}=0.07, \mathrm{p}=0.937\right)$ or stimulus $\left(\mathrm{F}_{(4,60)}=0.81, \mathrm{p}=0.526\right)$. A post hoc Bonferroni test indicated FLI was lower in sections collected at $-3.52 \mathrm{~mm}$ post bregma (mean $=15.5$ \pm 2.9 ) as compared to the other two AP levels (mean difference to $-3.08=-19.7,-3.88=$ $-15.8, \mathrm{p}<0.05)$. This prompted us to keep the data for FLI across side averaged but analyze data in each section level for stimulus and strain differences with two-way ANOVAs. Again, no main or interaction effects for strain or stimulus were found (Table 7-1). Mean number of FLI neurons for each stimulus/strain group were individually calculated and displayed with Figure 7-2.

To determine which groups were responsible for the lower FLI at - $3.52 \mathrm{~mm}$ post bregma, one-way ANOVAs (AP level) were used to test each stimulus/strain group individually. Results of these analyses are shown in Table 7-2, including whether Mauchly's test of sphericity passed indicating whether a corrected F-ratio was used to determine significance. Although most of the $\mathrm{p}$ values from these tests were near the $a$ priori significance value $(\alpha=0.05)$, none recapitulated the effect of AP level found in the follow-up ANOVA.

\section{Experiment 7.2}

\section{Animals}

CB6-Tg(Gad1-EGFP)G42 transgenic mice bred with GFP expressed hemizygously on the gadl promoter (GAD67-GFP mice) allowed the identification of GABAergic neurons $198,273,274$. 12 GAD67-GFP mice were used for the GAD67 experiment (ages on stimulation d: $68-89 \mathrm{~d}$, mean $=77 \pm 3 \mathrm{~d}, 6$ male, 6 female). Sample sizes of groups were similar to those published in other studies ${ }^{198}$. 
Table 7-1. Results of ANOVAs at VTA AP levels.

\begin{tabular}{ccccccccc}
\hline & \multicolumn{2}{c}{ Strain } & & \multicolumn{2}{c}{ Stimulus } & & \multicolumn{2}{c}{ Strain X Stimulus } \\
\cline { 2 - 3 } Level & $\mathbf{F}^{*}$ & $\mathbf{p}-$ Value & & $\mathbf{F}^{*}$ & $\mathbf{p}-$ Value & & $\mathbf{F}^{*}$ & $\mathbf{p}-$ Value \\
\hline-3.08 & 0.36 & 0.552 & & 0.29 & 0.753 & & 0.60 & 0.553 \\
-3.52 & 3.24 & 0.082 & & 1.40 & 0.263 & & 0.04 & 0.965 \\
-3.88 & 1.02 & 0.322 & & 1.60 & 0.218 & & 0.48 & 0.622 \\
\hline
\end{tabular}

* df were 1, 30 for strain, 2, 30 for stimulus, and 2, 30 for their interaction

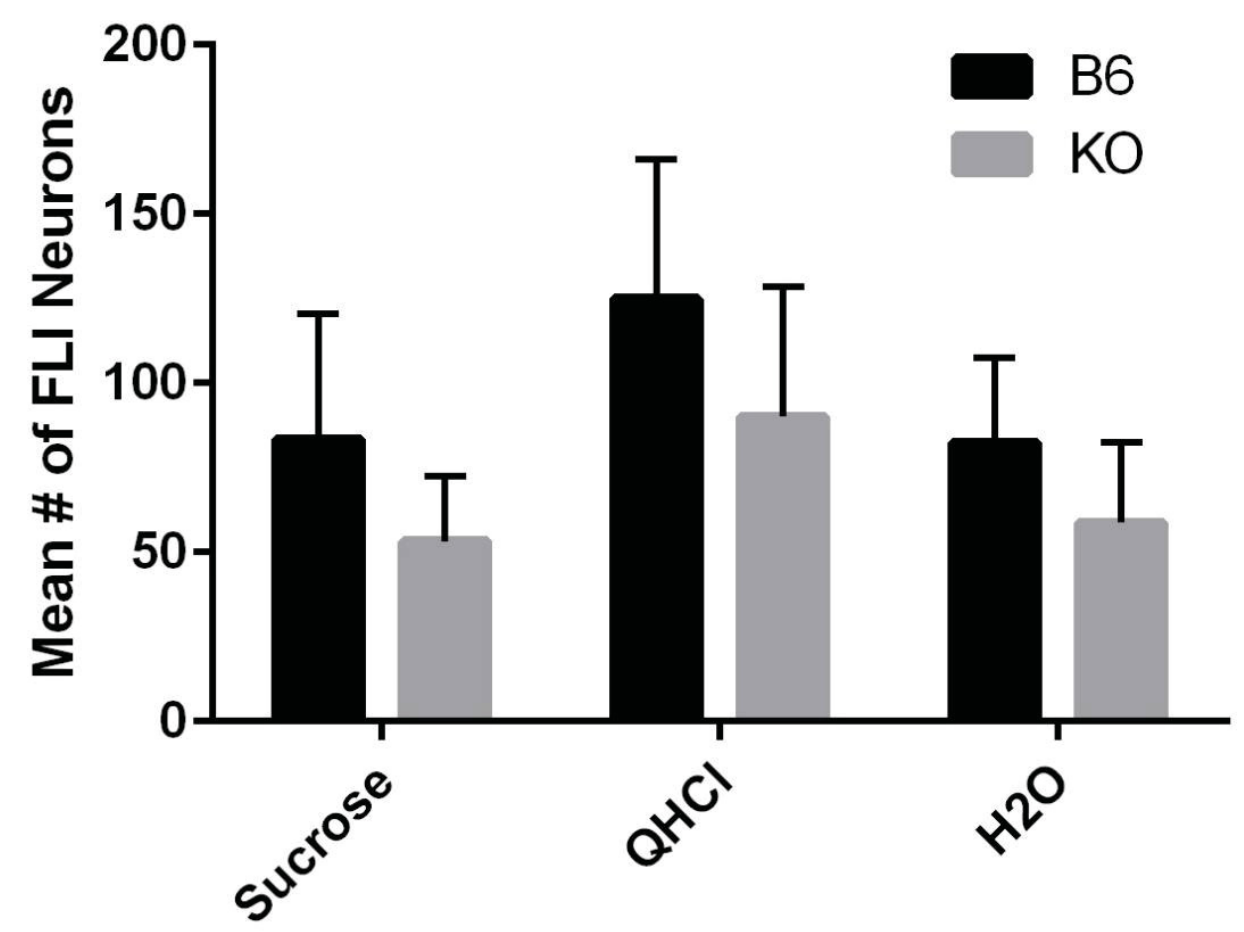

\section{Stimulus}

Figure 7-2. FLI in B6 and KO mice to taste stimuli.

Mice were i.o. infused with sucrose, $\mathrm{QHCl}$, and water. Data from sections were averaged across left and right; then, these section values were summed to produce a total number of FLI neurons in the VTA for each case. Cases in each strain/stimulus group were averaged to provide its mean number of FLI neurons. Apparent trending differences between FLI in B6 and KO mice were not statistically different. 
Table 7-2. Results of ANOVAs testing AP level for each strain/stimulus group.

\begin{tabular}{cccccc}
\hline Test & Mauchly's & $\mathbf{F}^{\mathbf{a}, \mathbf{b}}$ & $\mathbf{p}-$ Value & $\mathbf{G}^{\mathbf{c}} \mathbf{c}^{\mathbf{c}}$ & Significant $^{\mathbf{d}}$ \\
\hline Sucrose B6 & 0.023 & 2.31 & 0.185 & Yes & No \\
Sucrose KO & 0.511 & 2.28 & 0.152 & No & No \\
QHCl B6 & 0.943 & 1.30 & 0.315 & No & No \\
QHCl KO & 0.911 & 3.32 & 0.078 & No & No \\
H2O B6 & 0.015 & 3.81 & 0.104 & Yes & No \\
H2O KO & 0.618 & 2.38 & 0.143 & No & No \\
\hline
\end{tabular}

a. Greenhouse-Geisser corrected F-ratio was used if Mauchly's test of sphericity was not passed.

b. df were 1,5

c. Decision if Greenhouse-Geisser indicated to use corrected F-ratio.

d. Decision if p-value was significant. 


\section{Immunohistochemistry}

To stain TH and FLI, we stained the sections as in Chapter 5. Prior to the primary and secondary incubation steps, we used 3\% normal donkey serum for blocking (Jackson ImmunoResearch Laboratories). We incubated sections in mouse anti-TH (22941, ImmunoStar, Hudson, WI, USA, diluted to 1:5000) and rabbit anti-c-Fos (sc-52, lot \#F2510 Santa Cruz Biotechnology, diluted to 1:5000; sc-52, lot \#F3016 Santa Cruz Biotechnology, diluted to 1:500; or, ABE457, Millipore, diluted to 1:2000). Next, we used biotinylated donkey anti-rabbit IgG for secondary (Jackson ImmunoResearch, diluted to 1:1000) and DyLight 649-conjugated streptavidin for a fluorophore (SA-5649, Vector Laboratories, Burlingham, CA, USA, diluted to 1:1000). In the last steps, biotinylated donkey anti-mouse for secondary (Jackson ImmunoResearch, diluted to 1:1000), and streptavidin-Cy3 (Jackson ImmunoResearch, diluted to 1:1000).

\section{Histology}

As an anatomical reference to align the section's AP level, moving from rostral to caudal sections, the first section in which the ml touched the cp was identified as -3.64 mm (post) bregma. VTA subnuclei and the RRF (abbreviated as "subnuclei" from this point forward) were delineated using the Paxinos and Watson atlas, except for a few sections indicated by previous studies to contain VTA more anteriorly (see Lammel et al., $2008^{94}$ ). Detailed diagrams of the subnuclei delineated can be seen in Figure 7-3. TH-, GAD67-, and FLI-labeled neurons were plotted and quantified using ZEN software (black, black lite, and blue lite editions; Carl Zeiss, Thornwood, NY, USA). 14 representative sections, primarily at beginning and end points of subnuclei, were selected rostrocaudally $\mathrm{mm}$ relative to bregma: $-2.73,-2.97,-3.05,-3.29,-3.37,-3.45,-3.53,-3.69$, $-3.77,-3.85,-4.01,-4.25,-4.33$. Beginning and end points of subnuclei were as follows: PBP, -2.73 to -3.93 ; RLi, -3.05 to -3.93 ; IF, -3.05 to -3.8 ; PN, -3.37 to -3.77 ; RMTg, 3.53 to -4.41 ; RRF, -3.77 to -4.25 ; and, $\mathrm{CLi},-4.01$ to -4.41 .

Obvious landmarks were used to delineate subnuclei, including the interpeduncular fossa that appears medially $(\sim-3.05)$ and dorsal to the mammillary nucleus (MM), the detachment of the MM ( - -3.13), and expansion of the rostral subnucleus of the interpeduncular nucleus (IPN; beginning -3.21). In the most anterior sections, the medial and lateral pigmented parabrachial subnuclei (mPBP and 1PBP, respectively) were approximated in the atlas' unlabeled triangle within the LH, zona incerta (ZI), or posterior hypothalamus (PH). This area overlaps where Paxinos and Watson label the VTA's location in more posterior figures (Note: sagittal sections of Paxinos and Watson, Allen Brain Atlas, and Lammel et al. indicate the VTA extends more anterior than what is labeled in coronal sections of Paxinos and Watson; Lammel et al. indicates mPBP begins at this most anterior, but Allen Brain Atlas only appears to label lateral). The midline was approximated down the middle of the section using medial structures. The $\mathrm{cp}, \mathrm{SN}$, and $\mathrm{ml}$ were used to approximate the most lateral boundary. In more anterior sections a line was drawn ventrally from the $\mathrm{ml}$ to separate $1 \mathrm{PBP}$ from the $\mathrm{SN}$, but the more dorsal position of the $\mathrm{ml}$ in posterior sections demarcated this boundary. 

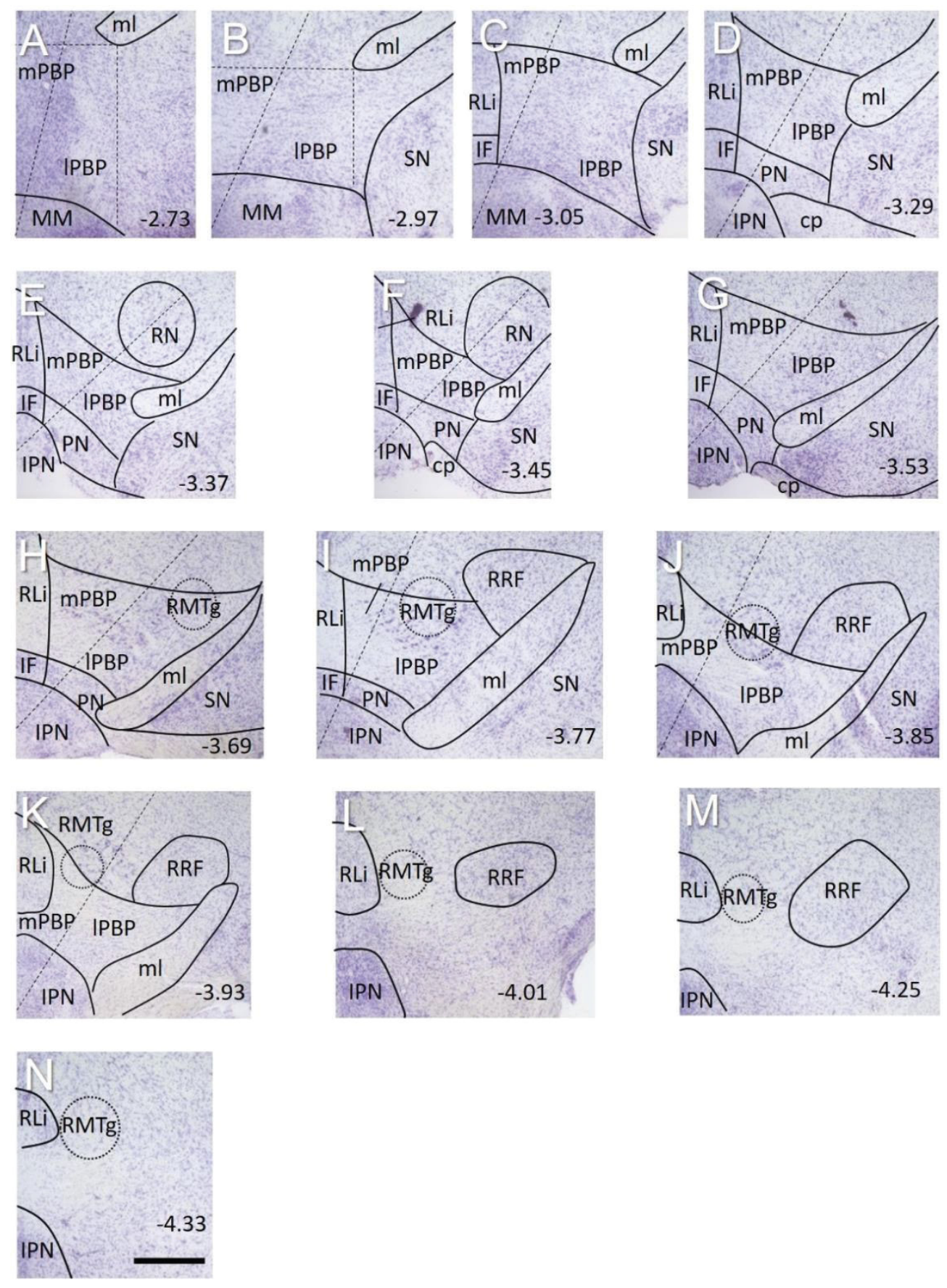

Figure 7-3. Semi-consecutive representative sections containing VTA subnuclei.

Sections were Nissl-stained with cresyl violet to reveal cytoarchitecture. Values in bottom right denote $\mathrm{mm}$ post bregma. Left boundary of images is midline. VTA subnuclei: PBP, pigmented parabrachial; RLi, rostral linear nucleus of the raphe; IF, interfascicular; PN, paranigral; RMTg, rostromedial tegmental; RRF, retrorubral field; $\mathrm{CLi}$, caudal linear nucleus of the raphe. All pictures at same magnification, scale bar in $\mathrm{N}$ $=25 \mu \mathrm{m}$. cp, cerebral peduncle; IPN, interpeduncular nucleus; ml, medial lemniscus; MM, mammillary nucleus; RN, red nucleus; SN, substantia nigra. 
A line drawn from the $\mathrm{ml}$ or tracing the $\mathrm{TH}+$ cells medially approximated the dorsal boundary. In anterior sections, a line drawn dorsal to the MM or the IPN or outlining the $\mathrm{TH}+$ cells from the lateral boundary approximated the ventral boundary. In posterior sections, the PBP ended and the RRF and CLi were approximated by outlining clusters of $\mathrm{TH}+$ cells corresponding to locations labeled in the Paxinos and Watson atlas. The PBP was split into medial and lateral areas by a line drawn from the center of the MM or IPN and dividing the scp/red nucleus $(\mathrm{RN})$ in half. The ventral disappearance of the ventral tegmental decussation was estimated as the ventral limit of the RLi and dorsal limit of the IF. The dorsal boundary of the IF and ventral boundary of the $\mathrm{ml}$ was used to estimate the $\mathrm{PN}$ in more anterior sections. Moving posteriorly, at the section when the distance between the $\mathrm{ml}$ and $\mathrm{cp}$ became shorter than the thickness of the IF, the thickness of the PN was estimated to be equal to that of the IF. The remaining boundaries of subnuclei, except the RMTg, were approximated according to Paxinos and Watson and/or where noticeable $\mathrm{TH}+$ cells clustered. The RMTg was approximated by drawing a circle around the cluster of GAD67+ cells which first appear in the 1PBP and, continuing posteriorly, appear to migrate medially. This GAD67+ cluster overlapped other subnuclei (e.g., PBP and RLi); thus, some overlap of cell counts occurred for this subnucleus only. However, RMTg data was analyzed separately from that of the remaining VTA and RRF.

When left and right side anatomy was noticeably asymmetrical, the difference in the AP level was estimated by multiplying the number of sections between each side's 3.64 section by $80 \mu \mathrm{m}$. If the difference in AP level was $<\sim 160 \mu \mathrm{m}$, medial and lateral subnuclei were matched as though no difference existed; if the difference was $\geq \sim 160$ $\mu \mathrm{m}$, medial and lateral subnuclei were treated as though they were $80 \mu \mathrm{m}$ apart (i.e., staggered medial and lateral subnuclei counts by 1 section).

\section{Quantification and Analysis}

Z-stack images were collected with confocal microscopy (equipment details in Chapter 5) through the sections with the aim of using the best image in the stack (i.e., one in which the entire VTA had cells visible) for counting. This resulted in roughly 2 to 6 images per stack through 10 to $15 \mu \mathrm{m}$ for each dry section. Images were collected at $20 \mathrm{x}$ magnification, and the optical section thickness ranged from about 1 to $3 \mu \mathrm{m}$.

Cells/nuclei were counted in ZEN by visualizing the images in double or single channels. DL cells/nuclei were marked first followed by single-labeled. Total counts were calculated by adding DL cells/nuclei to the single-labeled counts within the same categories. A cell/nucleus was only counted as valid if it was $>50 \%$ within the delineated area and not overlapping with another cell/nucleus counted in the same category (i.e., FLI, TH, or GAD67). A cell/nucleus was only counted as TH+ (cyan), GAD67+ (magenta), or FLI + (yellow) if $>75 \%$ of the structure was in the plane visualized and approximately $\geq 50 \%$ brightness/density relative to other visible structures of that same classification (judged by observer). Cells were only counted as $\mathrm{TH}+$, if a bright cell body was visibly enclosing a dark nucleus. To be counted as double or triple-labeled, criteria for 2 or 3 of the above labeling requirements had to be met for the same cell/nucleus, and where it would be expected, a change in color visualized had to occur (e.g., GAD67 and 
FLI overlap in the nucleus and blend colors). Quantification only included the right side, i.e., ipsilateral to the cannula. Bar graphs were made to compare DA and GABA cell counts/ratios between nuclei by AP level (see Figures S3C and S4E in Lammel et al., $2008^{94}$ ). To assess whether appetitive or aversive taste stimuli preferentially elicited activity in subnuclei or cell types, we analyzed data collected by projection, FLI positivity, and double labeling. ANOVAs were used to test for differences, followed by Bonferroni post-hoc tests where appropriate, and significance level was $\alpha=0.05$.

\section{Results 7.2}

Figure 7-4 depicts example micrographs of TH, GAD67, and FLI labeling found throughout the subnuclei. FLI double-labeling (with TH or GAD67) was also found, but double-labeling of TH and GAD67 was not. Both TH+ and GAD67+ cells varied significantly in shape and size and typically had processes visibly extending from their somas. FLI+ nuclei were typically circular or ovoid in shape. DL TH+ and FLI+ cells met both sets of criteria above as well as a FLI+ nucleus approximating the shape of the dark area within the TH+ cell body. DL GAD67+ and FLI+ cells met both of their criteria above; the overlap of the FLI+ nucleus and GAD67+ cell produced an off-white merged color. A priori criteria for DL TH+ and GAD67+ as well as triple-labeled cells were defined; however, neither was found in sections.

\section{TH and GAD67 Cells}

DA and GABA neurons were identified by fluorescently labeling TH and GAD67, respectively. We expected to find similar distributions of TH+ cells in specific subnuclei as were previously found ${ }^{94,156}$ as well as a distinct cluster of GAD67+ cells posteriorly in the RMTg ${ }^{166,197}$. The mean number of $\mathrm{TH}+$ cells found was 743 , and for GAD67+, 198. Although we expected cell-type labeling to differ by subnuclei, we did not expect a difference for stimulus group. We tested this for both cell types with ANOVAs (within-subjects for subnuclei, between-subjects for stimulus). As expected, TH differed for subnucleus $\left(\mathrm{F}_{(6,54)}=112.17, \mathrm{p}<0.001\right)$, but not stimulus $\left(\mathrm{F}_{(2,9)}=0.17, \mathrm{p}=0.851\right)$; GAD67 also differed for subnucleus $\left(\mathrm{F}_{(6,54)}=66.12, \mathrm{p}<0.001\right)$, but not stimulus $\left(\mathrm{F}_{(2,9)}=\right.$ $1.02, \mathrm{p}=0.4)$. Since the VTA varies substantially along its AP axis, a similar pair of analyses was conducted for AP level by reorganizing the data and repeating the tests. Again, we expected difference in subdivision (i.e., AP level), but not stimulus group. In this case, $\mathrm{TH}$ differed for AP level $\left(\mathrm{F}_{(13,117)}=33.75, \mathrm{p}<0.001\right)$, but not stimulus $\left(\mathrm{F}_{(2,9)}=\right.$ $0.17, \mathrm{p}=0.851)$; likewise, GAD67 differed for AP level $\left(\mathrm{F}_{(13,117)}=17.85, \mathrm{p}<0.001\right)$, but not stimulus $\left(\mathrm{F}_{(2,9)}=0.2, \mathrm{p}=0.821\right)$. $\mathrm{TH}+$ and GAD67+ cells were distributed throughout the VTA within its subnuclei and at varying AP levels as shown in Figures 7-5 and 7-6. Most of the TH+ cells were located within the 1PBP (Figure 7-5A). The second-highest counts of TH+ cells were in the RLi and RRF, followed by the PN, IF, mPBP, RMTg, and CLi (Figure 7-5B). Most of the GAD67+ cells were found in the RMTg, followed by the 1PBP and RRF (Figure 7-5C). Next to these, some GAD67+ cells were found in the mPBP, RLi, IF, PN, and CLi (Figure 7-5D). TH+ cells were the highest between -3.29 

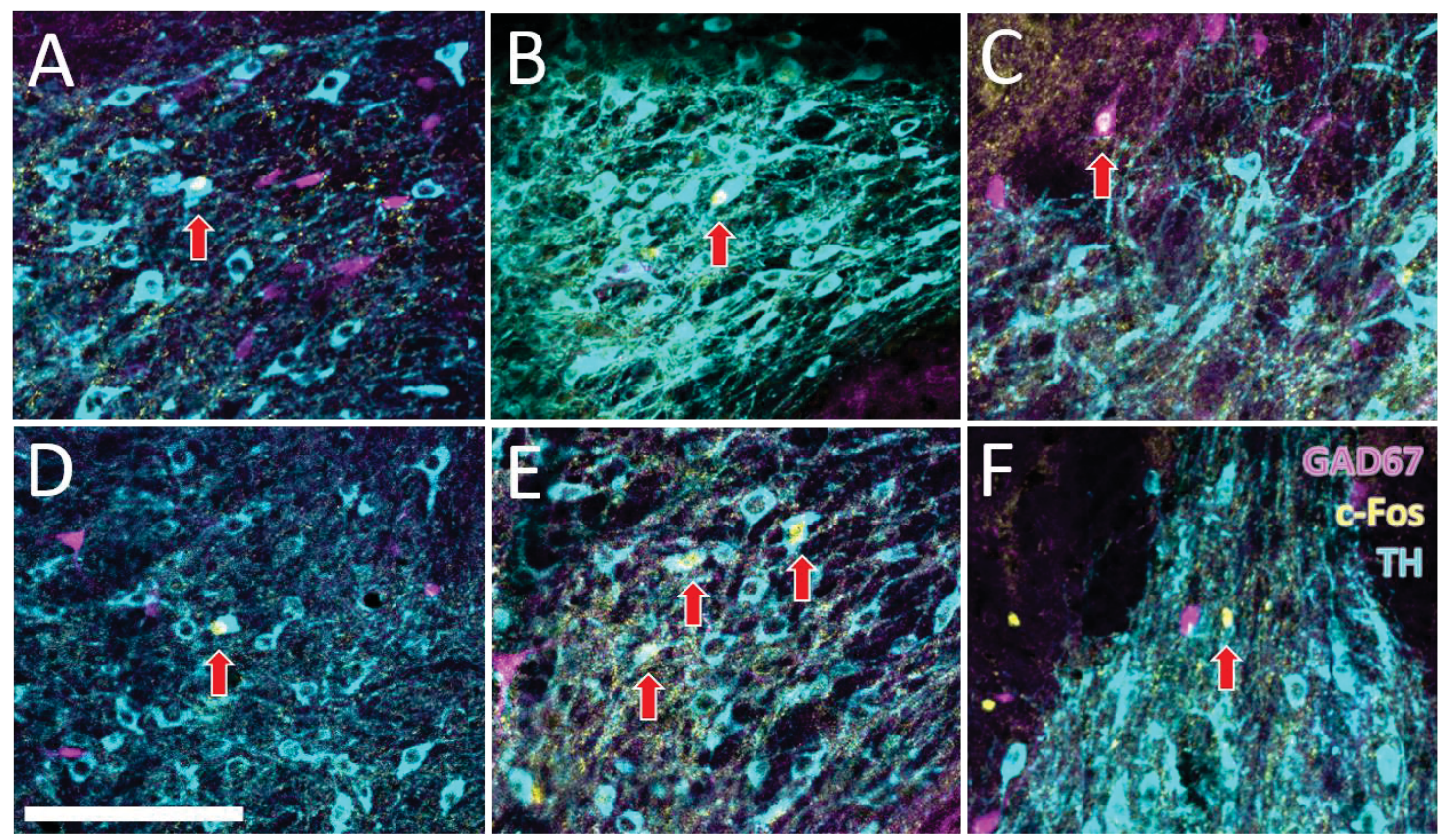

Figure 7-4. Examples of taste-elicited c-Fos in TH and GAD67 VTA neurons.

A-C. Sucrose elicited c-Fos. D-F. QHCl-elicited c-Fos. A, C, \& D. Lateral pigmented parabrachial subnucleus. B \& E. Border of pigmented parabrachial and paranigral subnucleus. F. Rostral linear nucleus of raphe. Cells with FLI are indicated with red arrows. Scale bar $=50 \mu \mathrm{m}$. 

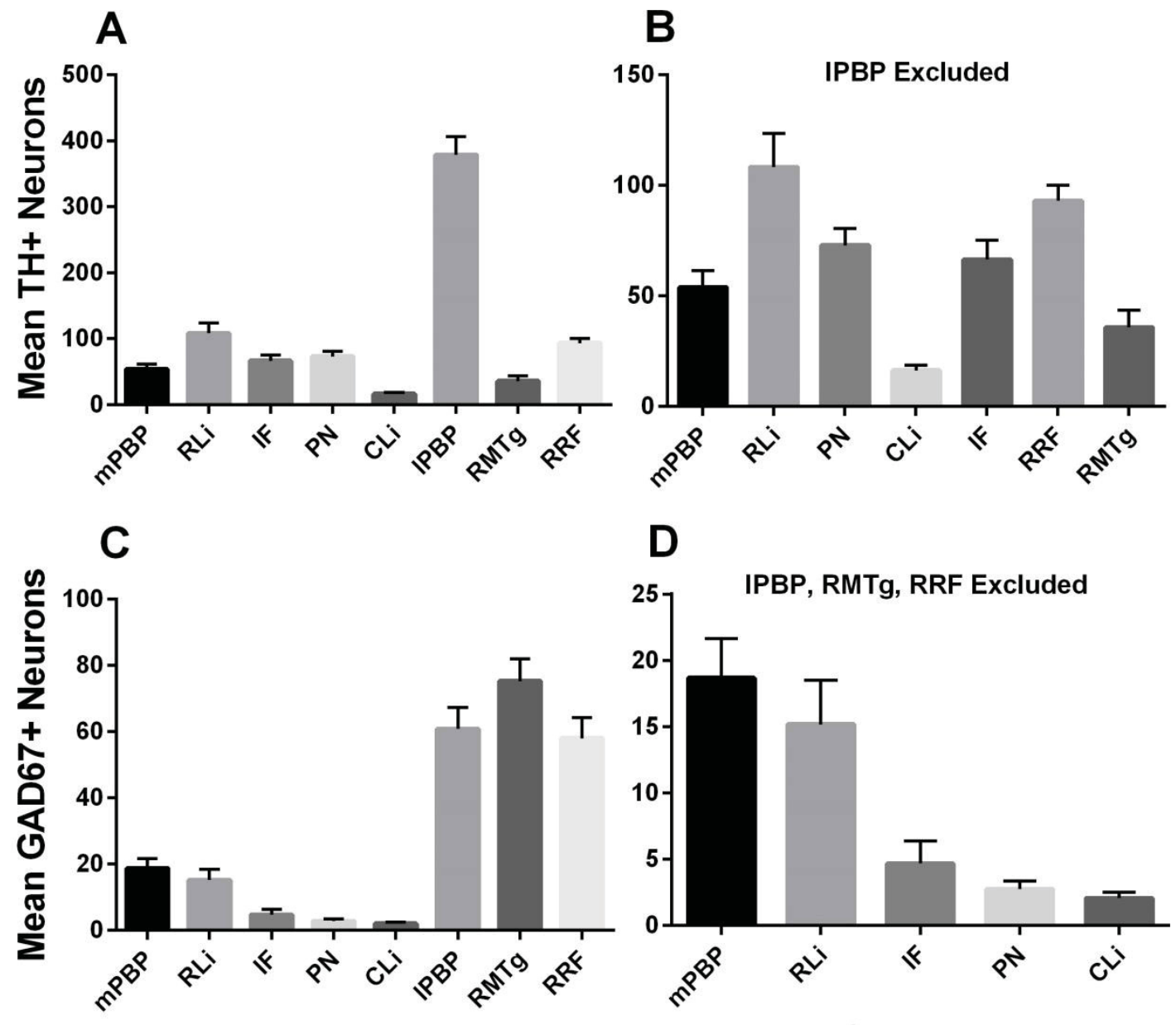

Subnucleus

Subnucleus

Figure 7-5. TH+ and GAD67+ cells in VTA subnuclei.

Depending on the subnucleus, different numbers of TH+ and GAD67+ cells were found, in part due to the differences in subnuclei volume and cell density. A. TH in the VTA: 1PBP had an overwhelmingly large proportion of TH. To give a better perspective of TH in VTA, a second graph was made excluding IPBP, B. B. In order of means from highest to lowest, TH neurons were found in the RLi, RRF, PN, IF, mPBP, RMTg, and CLi. C. GAD67 in the VTA: 1PBP, RMTg, and RRF had far more GAD67+ neurons in the VTA. Again, a second graph was made to allow perspective for the GAD67 in the remaining subnuclei, D. D. mPBP and RLi had fairly high GAD67, while the IF, PN, and CLi were sparsely populated with GAD67 neurons. 


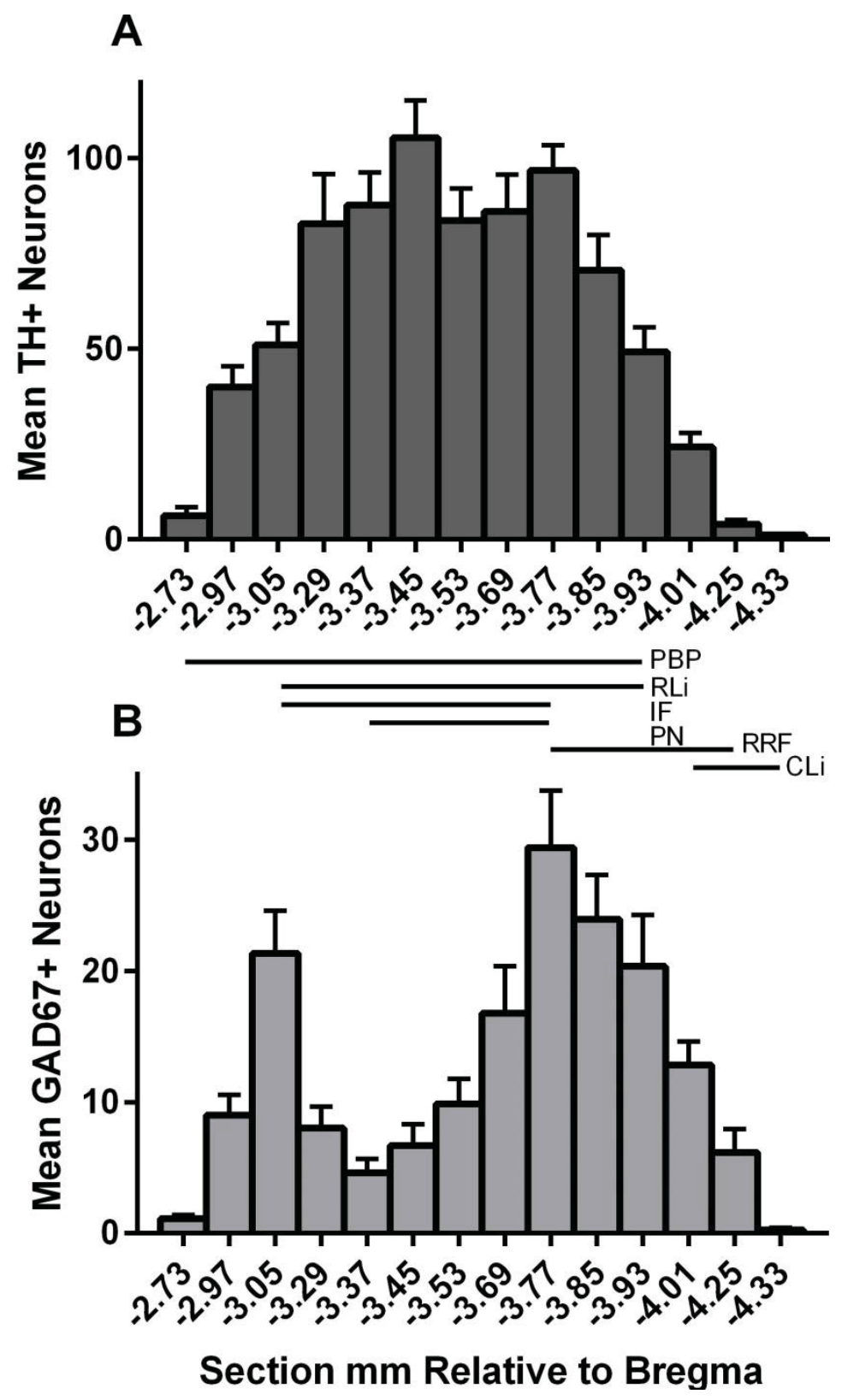

Figure 7-6. TH+ and GAD67+ cells in the VTA organized by AP level (mm relative to bregma).

A. $\mathrm{TH}+$ cells increase moving posteriorly until $\sim-3.29$ where they plateau until they decrease again beginning at -3.85 . B. GAD67+ cells form to peaks, the first of which begins its increase after -2.97 and drops back by -3.29 , only to increase again starting at 3.45 and decrease again at -3.85 . Lines between A and B indicate the subnuclei present at each anterior-posterior level. 
and $-3.85 \mathrm{~mm}$ post bregma (Figure 7-6A). GAD67+ cells formed two peaks at -3.05 and $-3.77 \mathrm{~mm}$ post bregma (Figure 7-6B).

The RMTg was recognizable as a roughly ovoid cluster of GAD67+ cells with boundaries that overlapped with several of the other subnuclei (e.g., RRF, 1PBP, CLi). Also noteworthy was this subnucleus' movement from a more lateral position to more medial moving posteriorly. ANOVAs to test for differences between stimulus groups revealed none occurred for $\mathrm{TH}\left(\mathrm{F}_{(2,9)}=0.43, \mathrm{p}=0.611\right)$ nor $\operatorname{GAD} 67\left(\mathrm{~F}_{(2,9)}=0.07, \mathrm{p}=\right.$ $0.935)$. A peak in $\mathrm{TH}+$ cells was seen at $-3.77 \mathrm{~mm}$ post bregma (Figure 7-7A), and GAD67+ cells were fairly stable from -3.53 to $-4.01 \mathrm{~mm}$ post bregma but dropping off more posteriorly (Figure 7-7B).

\section{FLI and Double-labeling}

FLI was measured in the subnuclei and cell types (DA, GABA) after stimulating them with sucrose, $\mathrm{QHCl}$, and water (control). Differentials in FLI were expected (either by subdivision [i.e., subnuclei, AP level], by cell type, or some combination of these) on the basis of taste stimulus group. FLI measurements alone in the subnuclei and at several AP levels did not pass normality tests. Hence, "FLI ratios" were calculated (FLI of subdivision / total FLI in VTA) and analyzed. The majority of the FLI ratios were distributed normally around means and passed normality tests; thus, parametric statistical tests were used on the calculated FLI ratios. Two-way repeated measures ANOVAs were used to test for differences in subnucleus and AP level as within-subjects variables and for stimulus group as a between-subjects factor. Differences in subdivision were expected, but these would more likely be due to differences in volumes and raw counts of cells than to actual differences in responsiveness to stimuli across subnuclei; we also hypothesized a differential in responses to taste stimuli may occur in subdivisions, which would be indicated by significant taste stimulus or stimulus $\mathrm{X}$ subdivision effects. Subnucleus was a significant main effect $\left(\mathrm{F}_{(6,54)}=50.5, \mathrm{p}<0.001\right)$, but no difference was found for stimulus group $\left(\mathrm{F}_{(2,9)}=2.92, \mathrm{p}=0.105\right)$. Further, no difference was found for the subnuclei $X$ stimulus interaction effect $\left(F_{(12,54)}=1.47, p=0.165\right.$; Figure 7-8). No difference was found for the stimulus group with the data organized by AP level either $\left(\mathrm{F}_{(2,9)}=0.05, \mathrm{p}=0.953\right)$. However, FLI in AP levels was different $\left(\mathrm{F}_{(13,117)}=25.7, \mathrm{p}<\right.$ $0.001)$, and the AP level $X$ stimulus group interaction was significant $\left(\mathrm{F}_{(26,117)}=1.95, \mathrm{p}=\right.$ 0.009; Figure 7-9). Follow-up Bonferroni tests revealed the section difference to be due to FLI ratios at -3.85 and -3.93 post bregma, which were higher $(\mathrm{p}<0.05)$ to $\mathrm{QHCl}(-$ 3.85 mean $=0.131 ;-3.93$ mean $=0.153)$ than to sucrose $(-3.85$ mean $=0.061 ;-3.93$ mean $=0.073)$ and water $(-3.85$ mean $=0.087 ;-3.93$ mean $=0.066)$. Data in sections at -3.85 and -3.93 were extracted for further tests to check for differences to taste stimuli in their subnuclei, but no differences were found.

FLI occurred in both TH and GAD67 cells, but no cases of TH + GAD67 or triple-labeling were found. Labeling of FLI, TH, and GAD67 likely influence the counts of TH + FLI and GAD67 + FLI DL cells; thus, this data was analyzed both for differences in DL cells as well as by calculating ratios of DL cells to each of the 

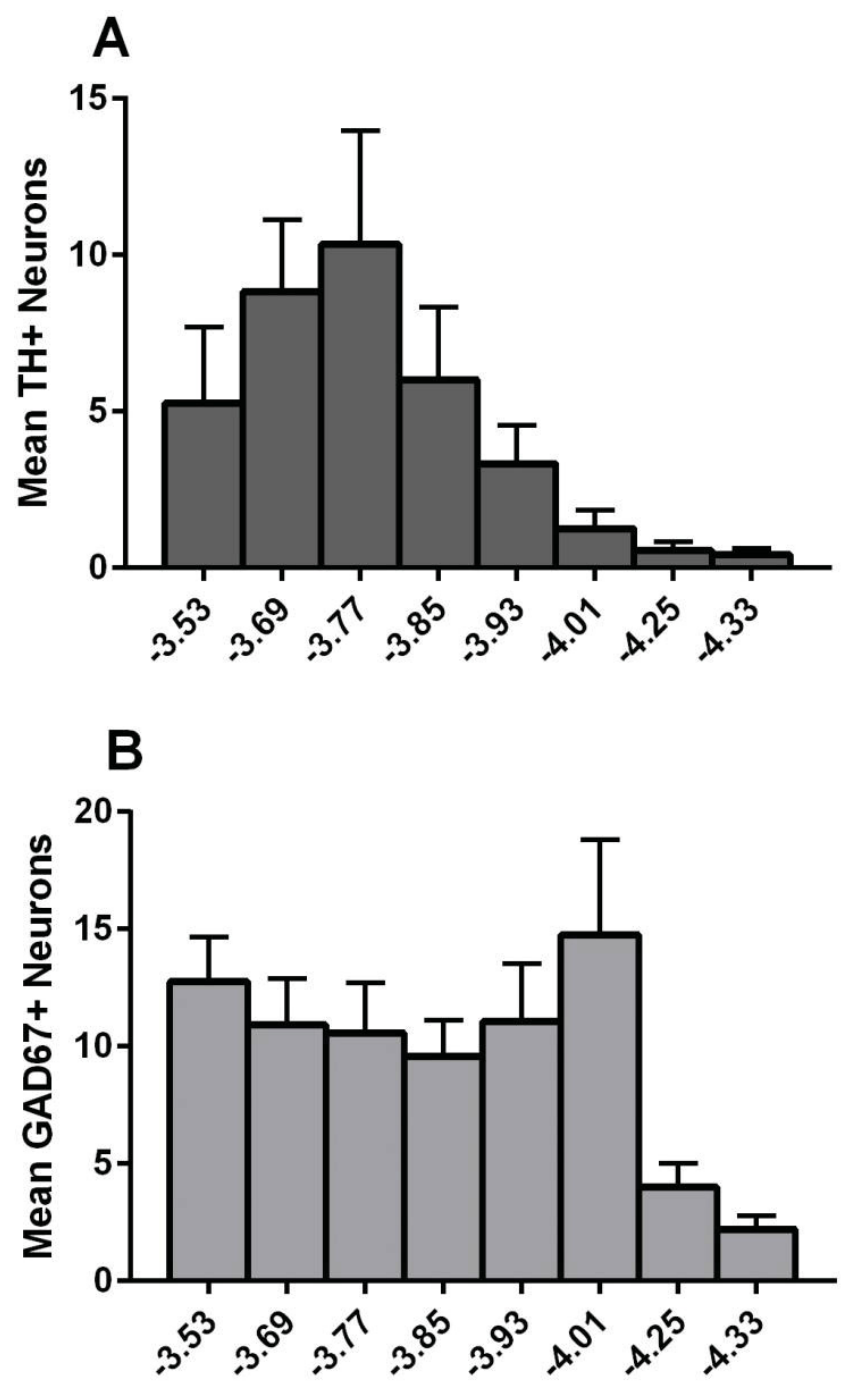

Section mm Relative to Bregma

Figure 7-7. TH+ and GAD67+ neurons in the RMTg subnucleus of the VTA.

A. $\mathrm{TH}+$ cells form a peak beginning their increase at -3.69 to decrease at -3.85 . B. GAD67+ cells are stable moving posteriorly through the RMTg until -4.25 when they drop. 


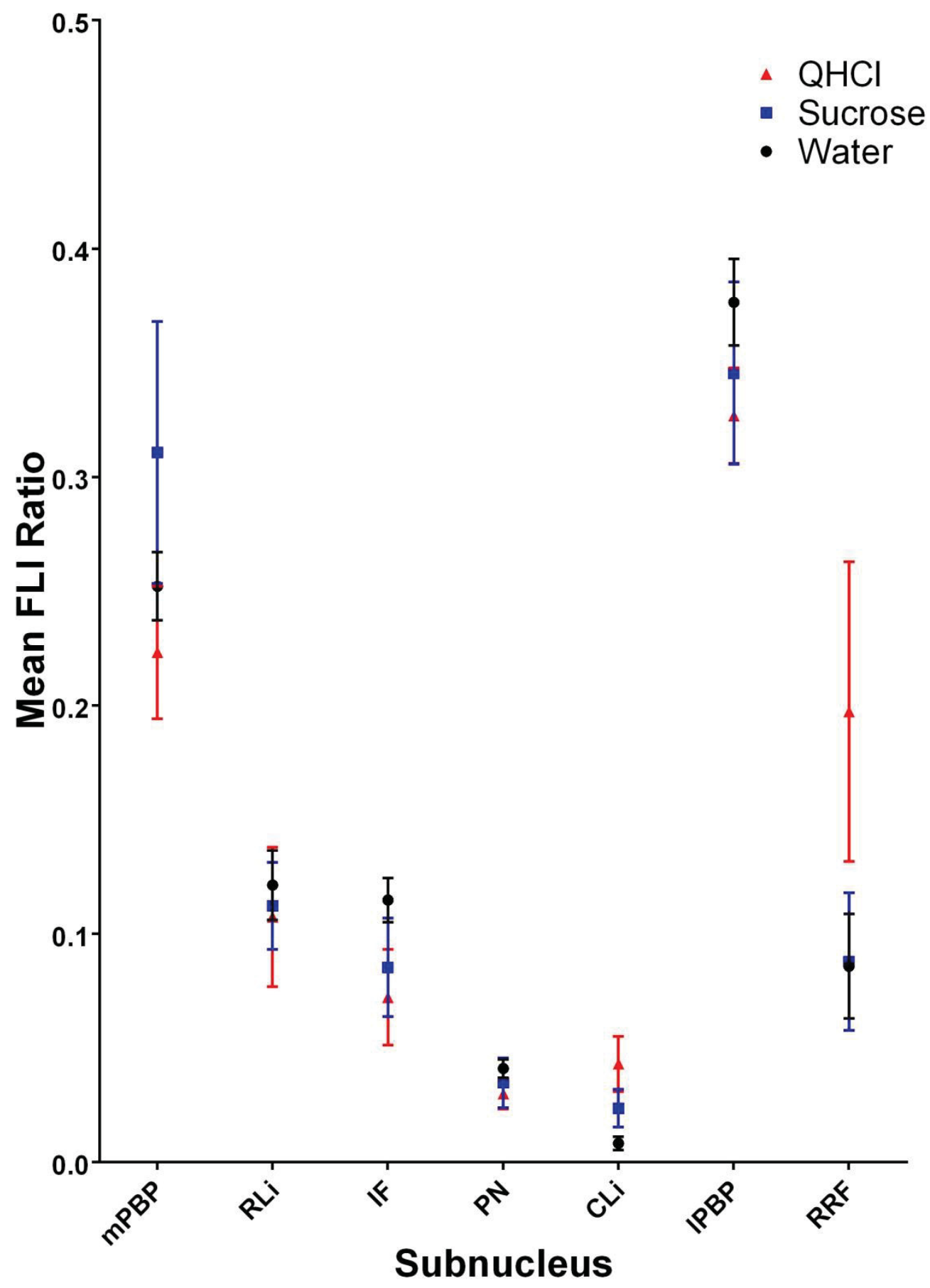

Figure 7-8. FLI ratios in VTA subnuclei to taste stimuli.

FLI ratio varied significantly across subnuclei $(\mathrm{p}<0.001)$ likely reflecting changes in subnucleus volume and cell density. However, no differences were found between taste stimulus group (water, sucrose, $\mathrm{QHCl}$ ) or its interaction with subnucleus. 


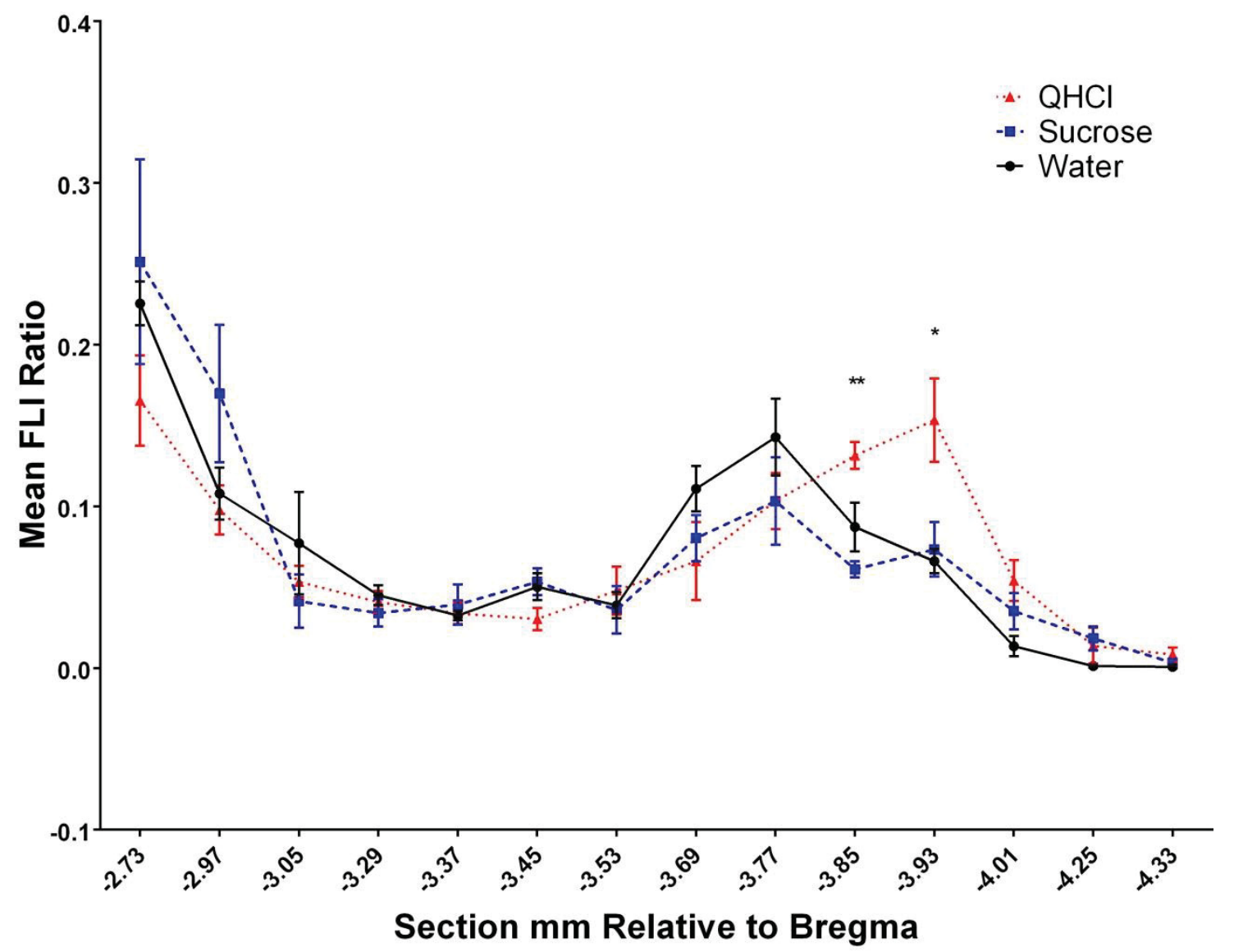

Figure 7-9. FLI ratios to taste stimuli in the VTA by anterior-posterior level.

Variation in FLI ratio across AP level was significant, but this was probably due to the area and cell density at each level. Taste stimulus was not a significant factor; however, the AP level X stimulus group interaction was significant $(\mathrm{p}=0.009)$. Further, FLI ratio to $\mathrm{QHCl}$ was found to be significantly higher than water or sucrose in the sections at 3.85 and -3.93 . Asterisks denote significance level: $* \mathrm{p}<0.05,{ }^{* *} \mathrm{p}<0.005$. 
constituents (e.g., TH + FLI counts in each subdivision [i.e., subnucleus, AP level] were divided by that subdivision's TH and analyzed, then by that subdivision's FLI and analyzed separately). Differences in subdivisions were expected as well as the possibility that some differential may occur in cell types to taste stimuli. Tests for sphericity failed, and Greenhouse-Geisser corrected F-ratios were used for determining significance. Results from the analyses of the TH + FLI double-label counts are presented in Figure 7-10. The effect of subnucleus was significant $\left(\mathrm{F}_{(6,54)}=3.95, \mathrm{p}=0.02\right)$, but stimulus group $\left(\mathrm{F}_{(2,9)}=0.59, \mathrm{p}=0.576\right)$ and the subnucleus $X$ group interaction $\left(\mathrm{F}_{(12,54)}=0.76, \mathrm{p}\right.$ $=0.606)$ were not (Figure 7-10A). When the data was reorganized into AP level (Figure 7-10B), similar results were found with a significant effect of level $\left(\mathrm{F}_{(13,117)}=3.95, \mathrm{p}=\right.$ $0.011)$ and acceptance of null hypotheses for stimulus $\left(\mathrm{F}_{(2,9)}=0.59, \mathrm{p}=0.576\right)$ and the interaction $\left(\mathrm{F}_{(26,117)}=1.38, \mathrm{p}=0.243\right)$. The effects of subdivisions in these cases can be explained by differences in the number of neurons as the volume or cell density of a subdivision changes. Analyzing ratios of double-label counts with respect to their constituents allows a test which compensates for this; thus, their significant subdivision effects are more meaningful. When TH + FLI counts were divided by FLI, subnucleus remained significant $\left(\mathrm{F}_{(6,54)}=4.39, \mathrm{p}=0.022\right)$ and an effect of stimulus group was found $\left(\mathrm{F}_{(2,9)}=4.51, \mathrm{p}=0.044\right)$. However, no interaction of subnucleus $\mathrm{X}$ group was found $\left(\mathrm{F}_{(12}\right.$, $\left.\left.{ }_{54}\right)=1.61, \mathrm{p}=0.206\right)$. When $\mathrm{TH}+\mathrm{FLI}$ counts were divided by $\mathrm{TH}$, subnucleus was again found to be significant $\left(\mathrm{F}_{(6,54)}=6.74, \mathrm{p}=0.002\right)$. Although the stimulus group for this adjustment was not significant $\left(\mathrm{F}_{(2,9)}=1.21, \mathrm{p}=0.343\right)$, the subnucleus $\mathrm{X}$ group interaction was $\left(\mathrm{F}_{(12,54)}=3.61, \mathrm{p}=0.01\right)$. These results suggest responses to taste stimuli vary in subnuclei but do so in a way relative to their overall FLI and TH expression. While adjusting for FLI revealed a difference in VTA TH + FLI expression to taste stimuli, adjusting for TH suggested the differences were within individual subnuclei. Bonferroni post hoc analyses were conducted for both of the adjusted DL counts to determine which subnuclei and taste stimuli were responsible for the effects. The analysis of $\mathrm{TH}+\mathrm{FLI}$ relative to FLI data indicated a higher mean ratio to $\mathrm{QHCl}$ in the $\mathrm{RLi}$ and $\mathrm{CLi}$ (mean ratios $=0.4,0.33$, respectively) compared to water (mean ratio $=0.1,0$, respectively; $p<0.05$ ). Mean ratios of taste stimuli in the rest of the subnuclei were not found to be significantly different. The analysis of TH + FLI with respect to TH data indicated mean ratios in CLi for water (mean ratio $=0)$, sucrose $($ mean ratio $=0.09)$, and $\mathrm{QHCl}($ mean ratio $=0.17)$ were all significantly different $(\mathrm{p}<0.05)$; again, the other subnuclei showed no differences. Together, these results strongly suggest a differential response to taste stimuli in the VTA TH neurons, especially in those of the CLi subnucleus (Figure 7-11). Like the double- label counts, the data for both ratios were reorganized into AP level. When TH + FLI was adjusted by the FLI count, AP level was not significant $\left(\mathrm{F}_{(13,117)}=2.38, \mathrm{p}=0.101\right.$; Figure 7-12). Interestingly, an effect of stimulus group occurred $\left(\mathrm{F}_{(2,9)}=5.61, \mathrm{p}=0.026\right)$, confirming the overall result for VTA found when analyzing the subnuclei data. The interaction of AP level X stimulus group was not significant $\left(\mathrm{F}_{(26,117)}=0.97, \mathrm{p}=0.46\right)$. When $\mathrm{TH}+\mathrm{FLI}$ was divided by TH, AP level was significant $\left(\mathrm{F}_{(13,117)}=5.26, \mathrm{p}=0.021\right)$; however, the lack of significance in the counts with respect to FLI suggests this effect is partially stochastic and merely differed as the result of overall FLI differences. Stimulus group $\left(\mathrm{F}_{(2,9)}=1.27, \mathrm{p}=0.327\right)$ and the interaction of group and level $\left(\mathrm{F}_{(26,117)}=0.71, \mathrm{p}=0.57\right)$ were also not significant. A similar analysis of GAD67 + FLI DL data was conducted, and results are presented in 

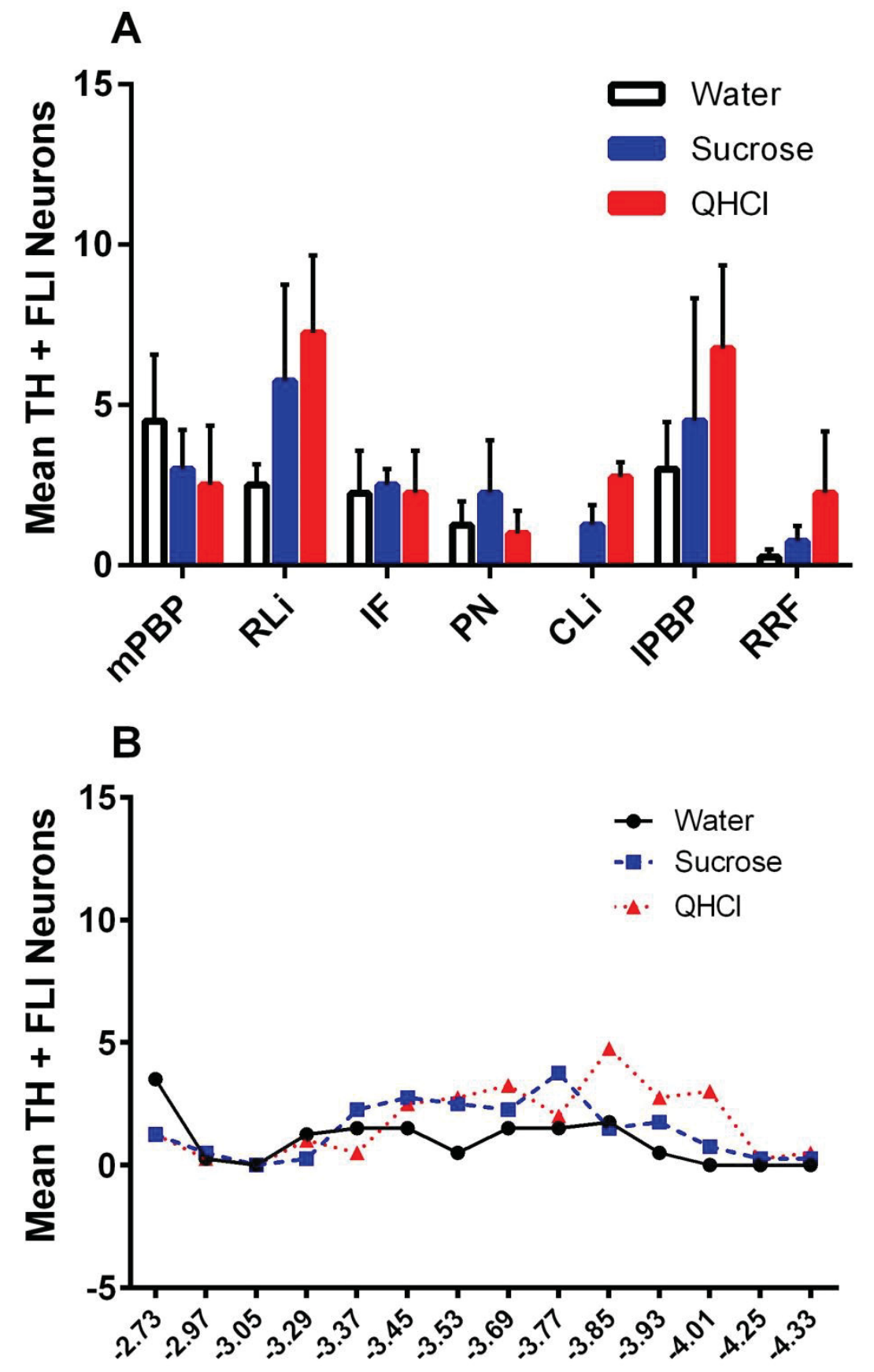

Section mm Relative to Bregma

Figure 7-10. TH + FLI counts to taste stimuli in the VTA.

Subdivision was a significant factor when analyzing double-labeled TH + FLI cells in the VTA's subnuclei (A) and at its AP levels (B); however, this was possible to the extent that subdivision volume and cell density varied. No differences were found in this raw data for stimulus group or subdivision $\mathrm{X}$ stimulus interaction. 

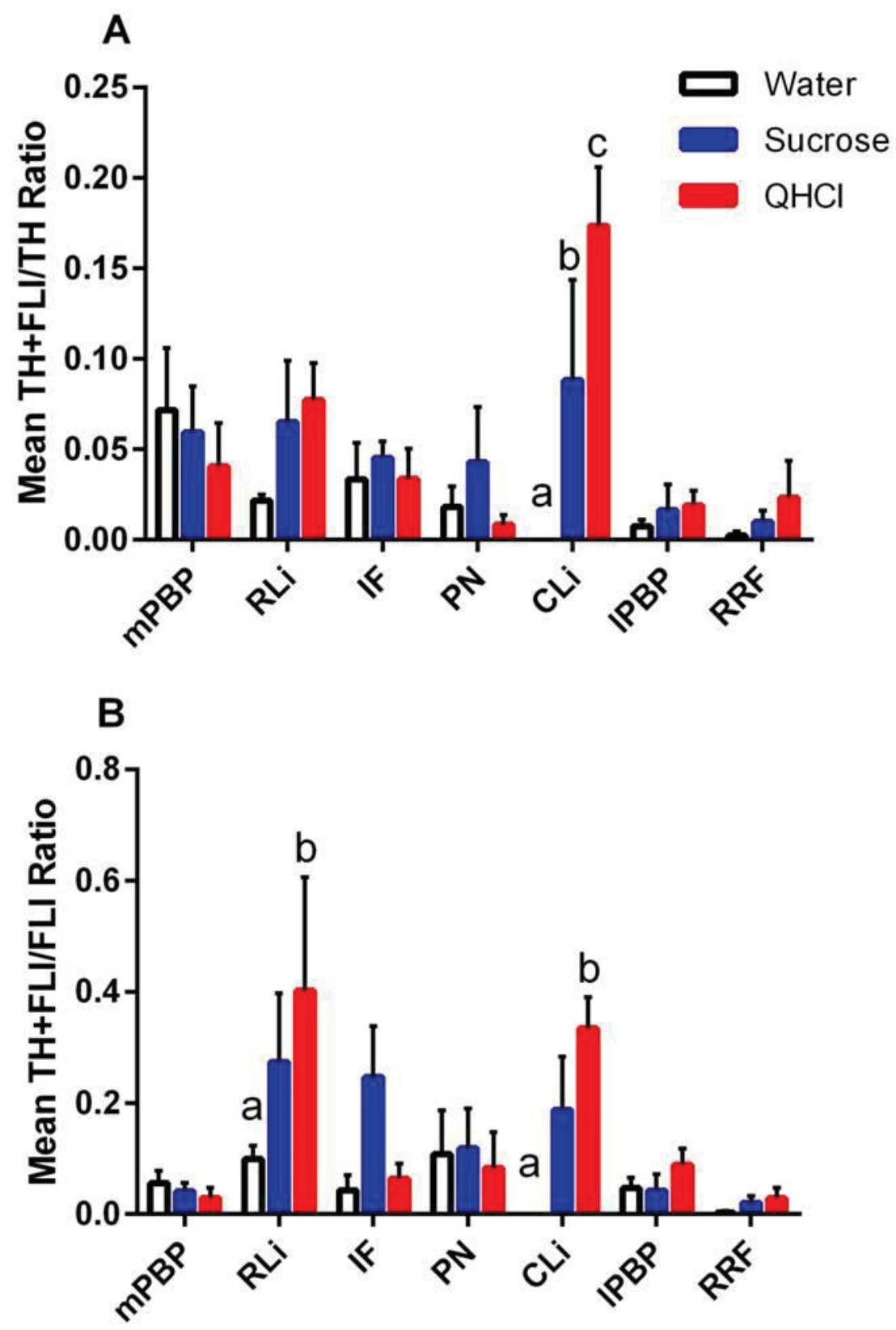

Subnuclei

Figure 7-11. TH + FLI ratios to taste stimuli relative to constituents in VTA subnuclei.

When double-labeled TH + FLI counts were analyzed as ratios of their constituents, differences remained significant within subnuclei and stimulus group became a significant factor. A. Relative to TH counts, TH + FLI cells were different for the 3 stimuli in CLi $(p<0.05)$. B. Relative to FLI, the RLi and CLi contained significant differences in double-labeling for water vs. QHCl. 


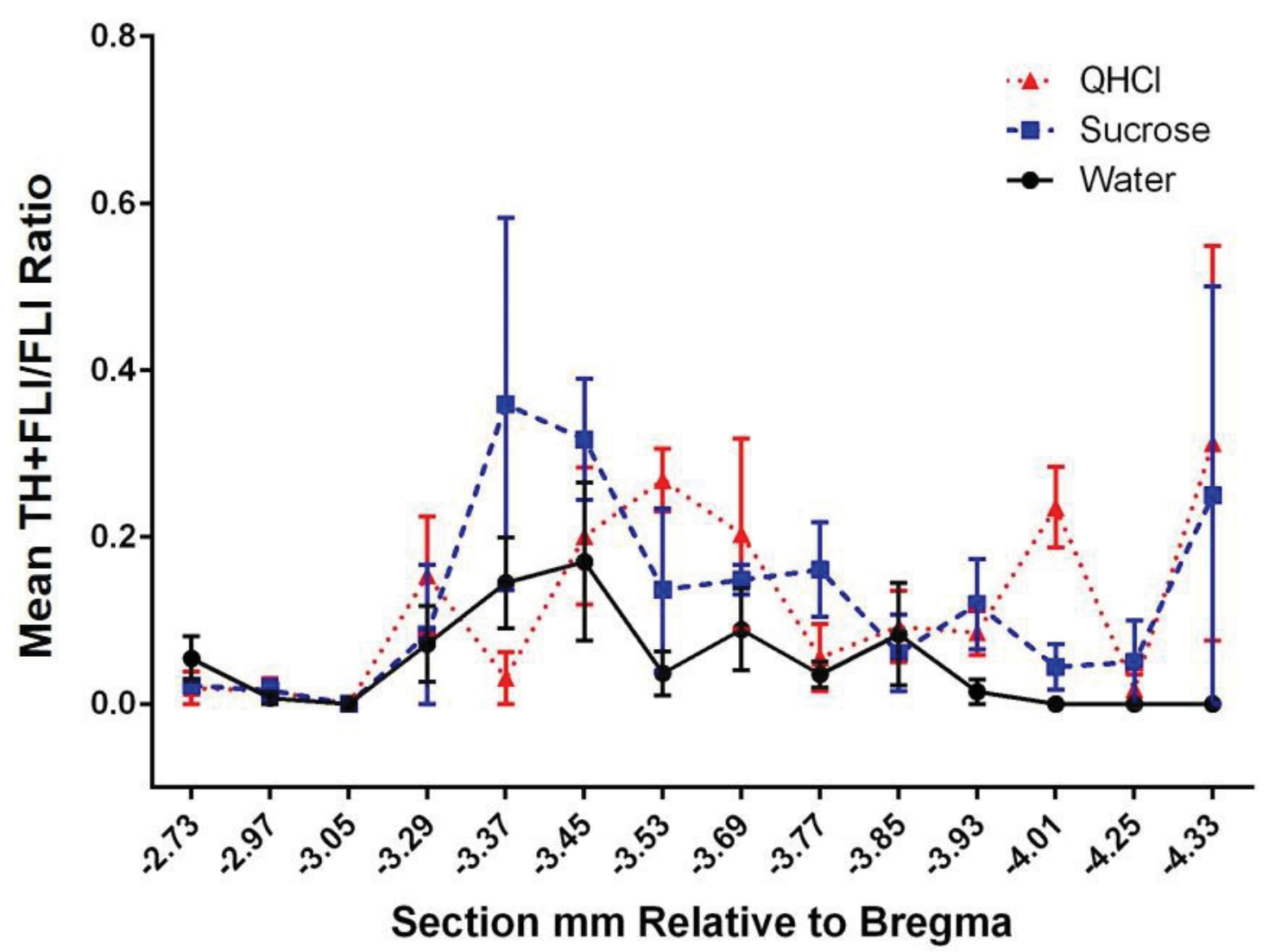

Figure 7-12. TH + FLI ratios to taste stimuli relative to constituents along AP axis of the VTA.

When TH + FLI was adjusted by the FLI count, AP level was not significant. An effect of stimulus group occurred, confirming the overall result for VTA found when analyzing the subnuclei data. The interaction of AP level X stimulus group was not significant. When the double-labeled counts were divided by TH (data not shown), no effects were found, suggesting the stimulus group effect occurred as a result elevated TH counts. 
Table 7-3. Significant effects were only found for subnucleus DL counts and GAD67 + FLI with respect to FLI. The former are expected to be due to variation in subnuclei volume and cell density, and the latter likely occurs from variation in GAD67+ neuronal count since the effect was not found when the data was adjusted for GAD67 cells.

The RMTg was segregated in the analyses because its area overlapped with other subnuclei. We conducted separate but similar analyses on FLI, double-labeling, and ratio data from the RMTg. The main difference in these was the only subdivision analyzed was AP level (it is only one subnucleus and it was not compared with statistical tests to the others). FLI ratios were calculated for the RMTg and tested for AP level and stimulus group effects with a two-way repeated measures ANOVA. AP level was significant $\left(\mathrm{F}_{(7}\right.$, $\left.{ }_{49)}=2.52, \mathrm{p}=0.027\right)$. Stimulus group $\left(\mathrm{F}_{(2,7)}=4.55, \mathrm{p}=0.054\right)$ and interaction effects $\left(\mathrm{F}_{(14,49)}=1.59, \mathrm{p}=0.116\right)$ were not. DL data for the RMTg was analyzed in a similar manner as the other subnuclei. In TH + FLI counts, neither AP level $\left(\mathrm{F}_{(7,49)}=1.33, \mathrm{p}=\right.$ $0.257)$ nor stimulus group $\left(\mathrm{F}_{(2,7)}=1.64, \mathrm{p}=0.261\right)$ were significant. A significant AP X stimulus interaction effect was found $\left(\mathrm{F}_{(14,49)}=1.95, \mathrm{p}=0.044\right)$, but this may reflect changes in the constituents instead. No other significant effects were found when the TH + FLI data were analyzed with respect to their constituents, nor were any found for GAD67 + FLI data (including adjusted values; see Table 7-4).

\section{Conclusions}

Results gained from measuring i.o.-evoked FLI in the VTA using DAB histology were inconsistent with the PBN study (Experiment 6.1) both in terms of stimulus effects and mouse strain (B6 vs. KO). Previously, microdialysis and electrophysiological studies showed that oral sucrose stimulation is followed by DA being released from neurons within the midbrain DAergic complex. With the VTA being one step away from the periphery compared to the PBN and receiving PBN input, we expected the VTA's i.o.evoked activity to reflect the PBN's. Deletion of the TRPM5 channel gene results in a loss of transduction in bitter and sweet TRCs. However, no FLI differences occurred in the VTAs between B6 and KO mice, suggesting the VTA may lack responsiveness to the taste aspects of these stimuli. Although we found FLI in the VTA to both sucrose and $\mathrm{QHCl}$, it was not greater than that resulting from water stimulation. We were also unable to show a differential in FLI to sucrose versus QHCl-tastes with opposite hedonic impacts. Several explanations for this lack of consistency between areas are possible: greater neuronal representation of visceral or TRPM5-independent gustatory inputs (discussed in Chapter 6) in the VTA; or conversely, PBN input conveying specificity about tastants vs. water may represent only a small part of the VTA activity following the type of behavior we measured here. It is also possible that FLI is not an optimal technique for estimating neuronal activation in this area relative to the PBN (see discussion of limitations in Chapter 9). The one effect found was lower FLI in the section at $-3.52 \mathrm{~mm}$ post bregma, but this was found in all 3 groups and could be due to differences in cell counts. An additional cross-cutting analysis of data from Experiments 6.1 and 7.1 was conducted to help explain the unexpected discord between the PBN and VTA FLI. Those results are discussed in Chapter 9. 
Table 7-3. Effects in VTA GAD67 + FLI double-labeled neurons.

\begin{tabular}{|c|c|c|c|c|c|}
\hline Subdivision* & DL/Denominator & Factor & df & $\mathbf{F}$ & p-Value \\
\hline \multirow[t]{3}{*}{ subN } & $\mathrm{DL}$ & subN & 6,54 & 5.33 & 0.015 \\
\hline & & stimulus & 2,9 & 0.99 & 0.410 \\
\hline & & subN X stimulus & 12,54 & 1.89 & 0.156 \\
\hline \multirow[t]{3}{*}{ subN } & FLI & subN & 6,54 & 5.32 & 0.011 \\
\hline & & stimulus & 2,9 & 0.91 & 0.438 \\
\hline & & subN X stimulus & 12,54 & 0.96 & 0.462 \\
\hline \multirow[t]{3}{*}{ subN } & GAD67 & subN & 6,54 & 0.50 & 0.570 \\
\hline & & stimulus & 2,9 & 1.13 & 0.372 \\
\hline & & subN X stimulus & 12,54 & 0.40 & 0.685 \\
\hline \multirow[t]{3}{*}{$\mathrm{AP}$} & DL & AP & 13,117 & 2.14 & 0.134 \\
\hline & & stimulus & 2,9 & 0.99 & 0.410 \\
\hline & & AP X stimulus & 26,117 & 1.13 & 0.374 \\
\hline \multirow[t]{3}{*}{$\mathrm{AP}$} & FLI & $\mathrm{AP}$ & 13,117 & 1.55 & 0.220 \\
\hline & & stimulus & 2,9 & 1.26 & 0.329 \\
\hline & & AP X stimulus & 26,117 & 0.93 & 0.492 \\
\hline \multirow[t]{3}{*}{ AP } & GAD67 & AP & 13,117 & 1.65 & 0.177 \\
\hline & & stimulus & 2,9 & 0.83 & 0.466 \\
\hline & & AP X stimulus & 26,117 & 0.72 & 0.682 \\
\hline
\end{tabular}

* subN, subnucleus; AP, anteroposterior level 
Table 7-4. Effects in RMTg double-labeled neurons.

\begin{tabular}{|c|c|c|c|c|c|}
\hline Cell Type & DL/Denominator & Factor & df & $\mathbf{F}$ & p-Value \\
\hline \multirow[t]{3}{*}{$\mathrm{TH}$} & $\mathrm{DL}$ & subN & 7,49 & 1.33 & 0.257 \\
\hline & & stimulus & 2,7 & 1.64 & 0.261 \\
\hline & & subN X stimulus & 14,49 & 1.95 & 0.044 \\
\hline \multirow[t]{3}{*}{$\mathrm{TH}$} & FLI & subN & 7,49 & 0.42 & 0.622 \\
\hline & & stimulus & 2,7 & 0.81 & 0.482 \\
\hline & & subN X stimulus & 14,49 & 0.66 & 0.600 \\
\hline \multirow[t]{3}{*}{$\mathrm{TH}$} & $\mathrm{TH}$ & subN & 7,49 & 0.77 & 0.449 \\
\hline & & stimulus & 2,7 & 0.24 & 0.793 \\
\hline & & subN X stimulus & 14,49 & 0.55 & 0.651 \\
\hline \multirow[t]{3}{*}{ GAD67 } & DL & $\mathrm{AP}$ & 7,49 & 3.07 & 0.058 \\
\hline & & stimulus & 2,7 & 0.39 & 0.692 \\
\hline & & AP X stimulus & 14,49 & 1.21 & 0.343 \\
\hline \multirow[t]{3}{*}{ GAD67 } & FLI & AP & 7,49 & 0.52 & 0.672 \\
\hline & & stimulus & 2,7 & 0.13 & 0.885 \\
\hline & & AP X stimulus & 14,49 & 0.48 & 0.815 \\
\hline \multirow[t]{3}{*}{ GAD67 } & GAD67 & $\mathrm{AP}$ & 7,49 & 0.59 & 0.552 \\
\hline & & stimulus & 2,7 & 1.27 & 0.340 \\
\hline & & AP X stimulus & 14,49 & 0.53 & 0.699 \\
\hline
\end{tabular}


The contradictory results between Park and Carr (1998) ${ }^{212}$, Kest et al. (2012) ${ }^{161}$, and Mungarndee et al. (2008) ${ }^{275}$ further suggests a need for nuance when discussing the communication of taste-to-reward nuclei, i.e., distinctions between oral and visceral origins must be considered. FLI elicited in the VTA of rats in the Park and Carr study ${ }^{212}$ was to meals of chow, sucrose, and vegetable shortening, a substance the rats found palatable and were allowed to fully ingest. Similarly, Kest et al. (2012) ${ }^{161}$ found elevated FLI in VTA TH neurons after rats consumed food pellets. In these two studies, one cannot determine whether FLI in the VTA was due to oral, visceral, or a combination of their input. However, rats in Mungarndee et al. (2008) ${ }^{275}$ had surgically-fistulated stomachs which emptied into troughs at the bottom of their cages, i.e., no viscerosensation occurred. With this manipulation, differences in FLI between water and taste stimuli could easily be assumed to be specific to taste, but none occurred in the VTA to $0.6 \mathrm{M}$ sucrose solution. These findings further support the idea that viscerosensation produces a strong, and perhaps the only, effect in the VTA to appetitive stimuli. Two final notes can be made that these studies used rats while ours involved mice; we used a PAP immunohistochemical protocol while the others used ABC methods. Neither of these methodological differences provides parsimonious explanations for the conflicting results; however, they may be all that is available to help explain why we found no difference in FLI in the VTA in our sucrose-stimulated mice, which should at the least have been elevated by the intact viscerosensation.

The TH+ and GAD67+ cells were seen distributed throughout the VTA and RRF as expected, including with significant counts of TH+ neurons in the PBP and PN subnuclei as were found in previous studies ${ }^{94,156}$. A cluster of GAD67+ cells, identified as the RMTg, were found posteriorly which started laterally and moved medially in more posterior sections, overlapping with other subnuclei. The identification of these midbrain areas allowed the investigation into their potential differential activity to taste stimuli.

FLI ratio in the AP data was significant in posterior sections, and TH + FLI data with respect to constituents showed significant activity in $\mathrm{CLi}$. These findings suggest a differential effect is occurring in these posterior TH+ cells to taste stimuli. More so, this effect appears to be due to responses to $\mathrm{QHCl}$, which somewhat conflicts with the narrative that palatable stimuli should be more effective. Instead, these data show the VTA response to an aversive stimulus, however, one with a likely potent salience (e.g., if bitter was equated with poison). The effect of TH + FLI with respect to FLI to taste stimuli remained a trend when DL counts were adjusted by $\mathrm{TH}$, suggesting the differential was only marginally reduced by accounting for the number of TH cells which could have potentially shown activity. Although few studies have attempted to narrow in on the differential of reward responses of individual VTA subnuclei (especially the RLi and $\mathrm{CLi}$ ), the RLi was previously found to have a role in reinforcing the behaviors involved in heroin use ${ }^{276}$. This is interesting in light of our finding that RLi TH + FLI was elevated in response to QHCl when data was adjusted by FLI. An AP X stimulus interaction effect in the RMTg TH + FLI data was found. However, this subnucleus is defined by its GAD67+ cell population; effects in GAD67 + FLI data would have been more impressive but were not found. Further, the lack of effect in the TH + FLI data with respect to TH or FLI further suggests this effect was stochastic. 
The difference in findings between the DAB and double-labeling data may be due to a couple of additional factors accounted for in the double-labeling data. For one, cell labeling, i.e., TH positivity, allowed a reference for neuronal count in the VTA. Second, measures and comparisons of individual subnuclei were made in Experiment 7.2. Also, the a, $\mathrm{p}$, and $\mathrm{t}$ distinction (essentially, an AP level factor) may not be as functionally important (at least not for taste stimuli) as subnuclei distinctions. Activity in GABA neurons appeared unimportant since few of these were FLI+. Anatomical studies of the VTA and forebrain, including tracing investigations using advanced methods, will help to identify VTA function in motivated behaviors like feeding. For example Beier et al. (2015) ${ }^{202}$ found an anterior cortex to VTA-DA to lateral NAc circuit to be reinforcing. De Luca (2014) ${ }^{239}$ was also able to show that DA release in forebrain areas that receive medial input (i.e., NAcc and mPFC) does not habituate. Thus, our finding that the DL TH + FLI occurred in two of the VTA medial subnuclei may be supported by this evidence of ML differential. While the lateral circuitry may be more important for the development of preference, the medial projections may inform the system about aversive stimuli. 


\section{CHAPTER 8. MODULATION OF FEEDING BY MANIPULATING THE VTA}

Our neuroanatomical approaches strongly suggested that taste stimuli varying in their hedonic valence, and in post-ingestive potency, elevated activity in neurons in the PBN and VTA, including PBN neurons that project to the VTA. A reasonable topic for subsequent inquiry was to investigate What effect might this circuit have on feeding? Hajnal and Norgren (2005) ${ }^{216}$ showed that oral application of sucrose results in DA release in the NAc, and lesioning the PBN diminished this release, indirectly implicating the PBN-VTA circuit as one which may mediate the affect associated with

consummatory and appetitive feeding behaviors. Considered with other observations that the PBN projected to the VTA ${ }^{80}$, including our own (Chapter 6), it seemed plausible that destruction of the PBN could have caused the decreased DA release in the NAc through direct disruption of this pathway. Other studies have shown DA release in the NAc to palatable stimuli ${ }^{127,239}$ suggesting the system for incentive salience is being activated ${ }^{10}$. Therefore, manipulating this pathway to increase or decrease that DA function should also affect feeding.

Activating the VTA-to-NAc neurons with DREADDs increased active lever pressing for sucrose pellets ${ }^{277}$, confirming this circuit's sufficiency in increasing urge for obtaining palatable food. A separate study showed activating the VTA DA neurons decreased average meal size and total food intake; however, feeding frequency increased ${ }^{241}$. Specifically activating the VTA-to-NAc was enough to reproduce the meal size and feeding frequency effects, but did not lower total food intake. Bilateral electrolytic lesions of the VTA decreased sucrose consumption, and lesioning the VTA with 6OHDA blocked the appetitive effects of i.p. midazolam (positive allostatic modulator of GABAR) or subcutaneous morphine (opioid agonist) ${ }^{211}$. Therefore, the VTA mediates feeding behavior, and to a certain extent the mechanism works via GABA and opioid systems. In fact, modulation of GABA receptors with intra-VTA muscimol or baclofen increased feeding (food pellets, Purina rat chow) ${ }^{251,252}$, effects which can be blocked by $\mathrm{GABA}_{\mathrm{B}}$ or opioid antagonism.

While some investigations showed systemic administrations of opioid and GABA modulators generally increased feeding and positive facial reactions ${ }^{278,279}$, others have attempted to use pharmacological manipulation to investigate the contribution of specific brain areas to taste-related feeding behaviors. For example, injecting the mu-opioid agonist DAMGO into the lateral PBN increases food intake ${ }^{280}$. Manipulations of the PBN via GABA mechanisms also modulates feeding ${ }^{237}$, and intra-PBN midazolam increased hedonic impact and increased feeding of a palatable stimulus ${ }^{238}$. However, results were not always consistent, as when sweeteners, such as saccharin, became aversive and total intake decreased when morphine was injected into the $\mathrm{PBN}^{234}$. Other manipulations of the PBN also have inhibitory effects on feeding such as activating a cluster of CGRP neurons in the el subnucleus of the PBN which project to part of the $\mathrm{CeA}^{73}$. 
Past studies have shown evidence that visceral and taste circuits each provide paths for different information to systems that modulate feeding, suggesting that factors regarding both palatability and caloric-content of stimuli need to be considered.

Responsiveness in the PBN to i.g. stimulation differs from i.o. ${ }^{67}$. Additional work has shown that viscerosensation and the transmission of caloric signals may be effective at driving consumption even if the ability to sense a palatable taste is disrupted. Sweet-blind trpm5 -/- mice gradually develop a preference to the caloric sweetener, sucrose, but not to the non-caloric sucralose ${ }^{246}$. The authors suggest these effects are due to post-ingestive feedback, which remain intact despite the disruption to the taste pathways. Moreover, separate circuits in brain areas other than the PBN and VTA were implicated in the communication of hedonic and nutritional information about sugar ${ }^{240}$.

To fortify the argument that the PBN's projection to the VTA serves to modulate feeding, we designed an experiment to isolate this circuit and measure the effects on feeding of activating it with DREADDs. This experiment proved methodologically challenging and yielded an inconclusive null result. Simplifying the question and methodology, we instead focused on what effect inhibiting the VTA with the GABA agonist, muscimol, had on palatability and appetite. Behavior was assessed using the MS160 lickometer. The advantage of measuring licking behavior is that both orosensory and post-ingestive factors of ingestion can be measured and analyzed (as opposed to longterm intake tests). The lick count (or rate) in response to stimuli delivered in this apparatus in brief trials ( $\leq 10 \mathrm{~s}$ ) by mice is considered a taste-salient behavior; on the other hand, the number of trials initiated in a session, as well as the cumulative intake function, are measures that reflect ingestion ${ }^{281}$.

\section{Experiment 8.1}

\section{Animals}

16 B6 mice were used for this experiment (ages on surgery d: $59-90 \mathrm{~d}$, mean $=$ $75 \pm 3$ d; 8 male, 8 female). Mice were randomly assigned to 1 of 3 groups: experimental, saline controls, and CNO controls. The conditions for the experimental group are explained in the upcoming sections. The saline control group received virus microinjections like the experimental, but i.p. saline replaced the CNO injections. The CNO control group did not receive microinjections, but $\mathrm{CNO}$ was i.p. injected.

\section{Viral Transfection}

Mice were anesthetized with ketamine/xylazine $(100 / 10 \mathrm{mg} / \mathrm{kg})$, and placed in a stereotaxic device. A midline incision was made to expose the skull. Burr holes with $\sim 1-$ $2 \mathrm{~mm}$ diameters were drilled over the injection site. Glass pipettes with inner diameters of $50-60 \mu \mathrm{m}$ were used with a picospritzer (52-302-900, General Valve Corporation, 
Fairfield, NJ, USA) for pressure injections. After the surgery, the incision was superglued or sutured closed, and mice were given antibiotic and buprenorphine.

To measure the potential effects of activating the PBN-to-VTA circuit on feeding behaviors (Figure 8-1), we used combined microinjections of a CRE-dependent DREADD and CRE-expressing vectors that targeted this pathway ${ }^{277,282}$. Other studies used similar methodology to target projections for DREADD activation ${ }^{277,283}$. Despite typically migrating anterogradely, adeno-associated viruses (AAV) have produced retrograde transfections when used in methods like the one proposed here ${ }^{284}$. Stock titers were diluted to 1 part stock in 4 parts deionized water. The diluted virus was successfully injected up to 1 week post-thaw. The stock titer of the rAAV was 5 X $10^{12}$. A $0.1 \mu \mathrm{L}$ bolus containing a vector for designer receptor exclusively activated by designer drug (DREADD; rAAV8-hSyn-DIO-hM3D(Gq)-mCherry) was pressure injected into the PBN bilaterally (AP: -5.4, DV 3.75, R\&L: +/- 1.2; +1 mm DV adjustment for brain depression). A $0.1 \mu \mathrm{L}$ bolus of CRE-recombinase expressing virus (AAV8-hSynmCherry-CRE) was pressure injected into the midline VTA (AP: -3.64, DV: 3.88, ML: 0; $+1 \mathrm{~mm}$ DV adjustment for brain depression), and its diffusion was expected to extend to bilateral limits without crossing into the SN. A fast rate of infusion (and thus, a temporally shorter, but increased pressure) was intended to overcome any inhibition by the heavily decussated VTA while the $\mathrm{ml}$ and scp were expected to limit diffusion into the $\mathrm{SN}$.

\section{Behavioral Apparatus}

A Davis MS-160 contact lickometer was used here and in the following experiment (8.2) for habituation, training, and testing procedures. The apparatus consisted of a plastic test chamber $(30 \times 14.5 \times 16 \mathrm{~cm})$ with a stainless steel front wall and mesh floor (DiLog Instruments; Tallahassee, FL, USA). For details of basic use in our lab, see Boughter et al. (2002) ${ }^{285}$, St. John and Boughter (2009) ${ }^{286}$, and Saites et al. $(2015)^{259}$; for the full scope of use in the field, the reader may reference other studies $240,255,256,287$. Where specified below, access to one or more bottles containing aqueous stimuli was provided through a small aperture in the front wall of the chamber, closeable via a shutter. Trials began and ended with the opening and closing of the shutter, respectively. Licks were detected and counted via a computer when a (undetectable) low current circuit was closed by the contact of a mouse's tongue with the steel of the bottle's sipper.

\section{Taste Solutions}

All tastants were prepared fresh daily using reagent grade chemicals (SigmaAldrich) dissolved in distilled water. Calorie-free taste stimuli were initially selected to prevent metabolic effects from interfering with outcome measures: $0.1 \mathrm{mM} \mathrm{QHCl}$ and 3 $\mathrm{mM}$ sucralose. 

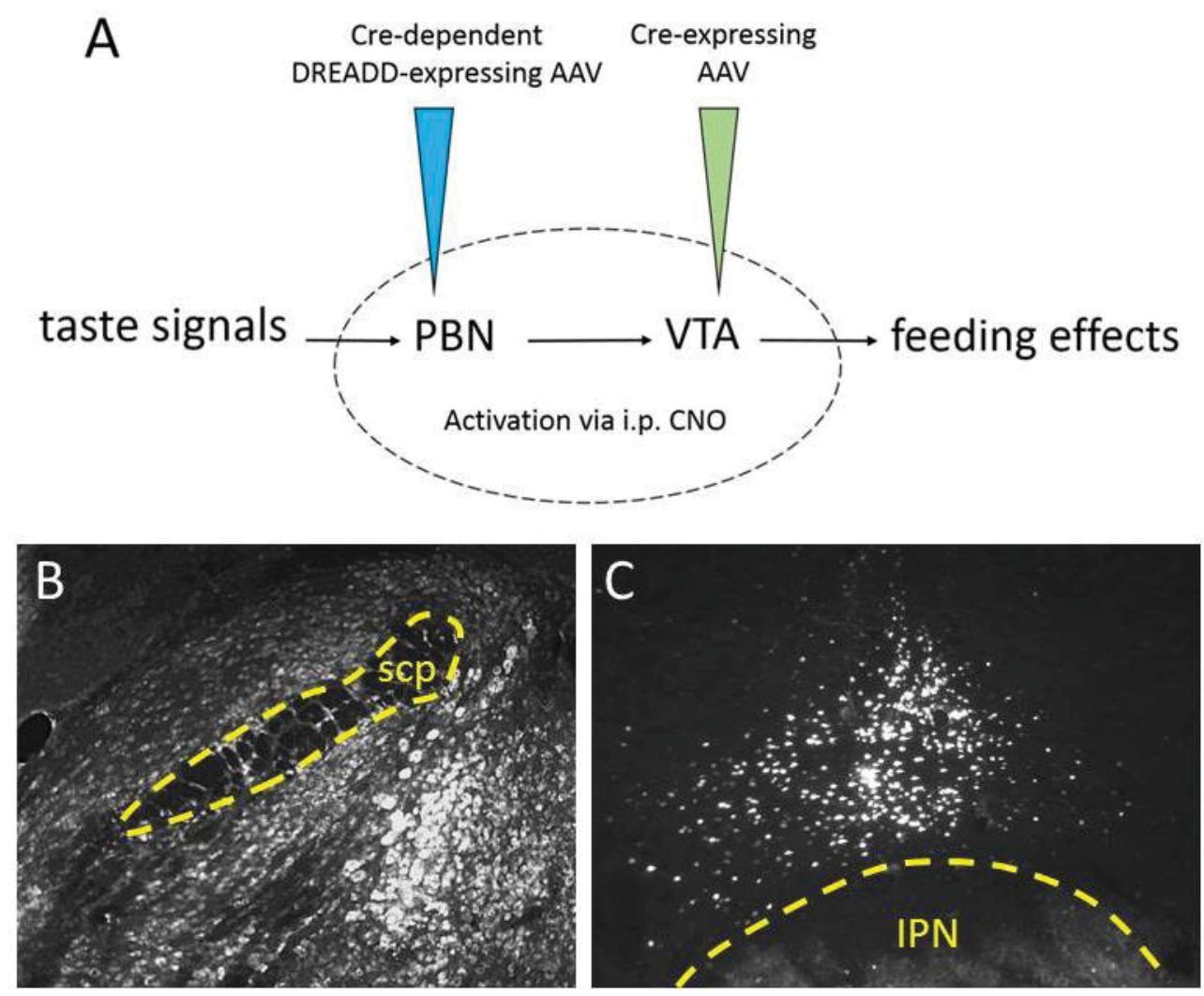

Figure 8-1. Targeted activation of the PBN-to-VTA circuit may affect feeding.

A. Schematic of the experimental design. Viral vectors were microinjected into the PBN and VTA, causing DREADD expression only in this circuit. Subsequent i.p. injection of CNO ostensibly activated this circuit which was expected to affect feeding. B \& C. Example microinjections of the mCherry-expressing AAVs into the PBN and VTA. IPN, interpeduncular nucleus; scp, superior cerebellar peduncle. 


\section{Training}

The entire training and testing schedule is summarized in Table 8-1. Viral microinjections were done in the first week of the experiment, and mice were allowed to recover at least 3 days before training began. The training started with a $5 \mathrm{~min}$ "habituation" session in the chamber without stimuli. Sipper tube training sessions consisted of a single, 20 min access trial to water. Trial training sessions included $27-5$ $\mathrm{s}$ trials separated by $7.5 \mathrm{~s}$ inter-trial intervals. After the shutter opened, a given trial could be initiated by a lick. If the mouse did not lick within 2 min of the shutter opening, the shutter closed and reopened after the inter-trial interval. These events were noted as skipped or missed trials. Finally, a single session ended after 30 min regardless of whether the mouse had completed all 27 trials. The training week (Week 2) included, consecutively: $1 \mathrm{~d}$ habituation, $1 \mathrm{~d} 20$ min water, and $3 \mathrm{~d}$ trial training. Subsequent weeks (Weeks 3 and 4) started with $2 \mathrm{~d}$ trial training followed by pre-testing and testing. To habituate the mice to the stress of i.p. injections, saline was given before each training session starting the first day of trial training.

The viral incubation period overlapped with training but ended before testing began. Mice were placed on water restriction the first week: they only received $2 \mathrm{~mL}$ of water after the session, and only if they initiated at least 3 trials and licked at least 10 times during the session (no lick count per trial requirements were made; no water was provided, if mice did not meet the requirements). Mice were then placed on food restriction (no food) in Weeks 3 and 4 (except in rest periods beginning Fridays after sessions and ending Mondays before sessions), including when pre-test and test data were collected.

\section{CNO}

A volume of $2.5 \mu \mathrm{L}$ sterile DMSO was used to dissolve $0.5 \mathrm{mg}$ CNO. Aliquots of $\mathrm{CNO}$ in DMSO were stored at $-10{ }^{\circ} \mathrm{C}$ and prepared freshly before use. On test days, $5 \mathrm{~mL}$ sterile saline was added, and the solution was filtered with a $0.2 \mu \mathrm{m}$ sterile syringe filter. The final concentrations of DMSO and CNO were $0.5 \%$ and $0.1 \mathrm{mg} / \mathrm{mL}$, respectively. $\mathrm{CNO}$ was administered i.p. 15 min prior to testing. From the body weight (BW) measured, the volume calculated was based on $1 \mathrm{mg} / \mathrm{kg}$ dose. Following the 19 day incubation period, i.p. injection of CNO ostensibly, selectively activated the PBN-toVTA circuit via DREADD-modulation, allowing measurement of any changes in palatability or appetite it mediates. The effects of CNO in DREADD-expressing mice could last up to 9 hours; however, appropriate viral titers and $\mathrm{CNO}$ dose supposedly optimized the behavioral effect's timing and minimized circuit desensitization ${ }^{288}$.

\section{Histology}

Effectiveness of DREADD-activation and cannula placements were checked via $a$ posteriori analyses. After DREADD testing was complete, mice were given an i.p. 
Table 8-1. PBN-to-VTA activation behavioral paradigm schedule.

\begin{tabular}{|c|c|c|c|c|c|c|c|}
\hline Week & Sunday & Monday & Tuesday & Wednesday & Thursday & Friday & Saturday \\
\hline 1 & & Microinject & Microinject & Microinject & Microinject & Microinject & rest \\
\hline 2 & rest & $\begin{array}{l}\text { habituate \& } \\
\text { water restrict }\end{array}$ & $\underset{\text { train }}{20 \min _{2} \mathrm{H}_{2} \mathrm{O}}$ & $\begin{array}{l}5 \mathrm{~s} \text { trials } \mathrm{H}_{2} \mathrm{O} \\
\text { train }\end{array}$ & $5 \mathrm{~s}$ trials $\mathrm{H}_{2} \mathrm{O}$ train & $\begin{array}{c}5 \mathrm{~s} \text { trials } \mathrm{H}_{2} \mathrm{O} \text { train } \& \\
\text { water replete }\end{array}$ & rest \\
\hline 3 & rest & $\begin{array}{l}5 \mathrm{~s} \text { trials } \mathrm{H}_{2} \mathrm{O} \\
\text { train \& food } \\
\text { restrict }\end{array}$ & $\begin{array}{l}5 \mathrm{~s} \text { trials } \\
\mathrm{H}_{2} \mathrm{O} \text { train }\end{array}$ & $\begin{array}{c}5 \text { s trials } \\
\text { sucralose, } \mathrm{QHCl} \\
\& \mathrm{H}_{2} \mathrm{O} \text { pre-test }\end{array}$ & $\begin{array}{c}5 \text { s trials sucralose, } \\
\mathrm{QHCl} \mathrm{\&} \mathrm{H}_{2} \mathrm{O} \text { pre- } \\
\text { test }\end{array}$ & $\begin{array}{l}5 \mathrm{~s} \text { trials sucralose, } \\
\mathrm{QHCl} \& \mathrm{H}_{2} \mathrm{O} \text { pre-test } \\
\text { \& food replete }\end{array}$ & rest \\
\hline 4 & rest & $\begin{array}{l}5 \mathrm{~s} \text { trials } \mathrm{H}_{2} \mathrm{O} \\
\text { train \& food } \\
\text { restrict }\end{array}$ & $\begin{array}{l}5 \mathrm{~s} \text { trials } \\
\mathrm{H}_{2} \mathrm{O} \text { train }\end{array}$ & $\begin{array}{c}5 \text { s trials } \\
\text { sucralose, } \mathrm{QHCl} \\
\& \mathrm{H}_{2} \mathrm{O} \text { test }\end{array}$ & $\begin{array}{l}5 \mathrm{~s} \text { trials sucralose, } \\
\mathrm{QHCl} \& \mathrm{H}_{2} \mathrm{O} \text { test }\end{array}$ & $\begin{array}{l}5 \text { s trials sucralose, } \\
\mathrm{QHCl} \& \mathrm{H}_{2} \mathrm{O} \text { test \& } \\
\text { food replete }\end{array}$ & rest \\
\hline
\end{tabular}

a. Week 1: Virus microinjections were done.

b. Mice were restricted in water and food consumption beginning in Week 2 on Mondays and ending on Fridays.

c. Habituate: Training started with a 5 min session in the chamber without stimuli to allow mice to acclimate.

d. 20 min $\mathrm{H}_{2} \mathrm{O}$ train: The second training session was 20 min with only a water bottle.

e. $5 \mathrm{~s}$ trials: Trial training, pre-test, and test sessions included $27-5 \mathrm{~s}$ trials separated by $7.5 \mathrm{~s}$ inter-trial intervals. After the shutter opened, a given trial could be initiated by a lick. If the mouse did not lick within 2 min of the shutter opening, the shutter closed and reopened after the inter-trial interval. These events were noted as skipped or missed trials. Finally, a single session ended after 30 min regardless of whether the mouse had completed all 27 trials.

f. More details may be found in the methods section. 
injection of $\mathrm{CNO}$ (saline controls received saline) and perfused $2 \mathrm{~h}$ later. Brains were collected and 4/16 (4/6 of experimental group) were sectioned as described previously. Sections in and around the AP level of the VTA and PBN were collected for the immunohistochemistry preparation. To label mCherry and c-Fos, we treated the sections as in General Methods, except the primary was already conjugated with Alexa Fluor 594 (no additional steps required) and blocking included 6\% bovine serum albumin (9048-468 , Sigma-Aldrich) in addition to 3\% normal donkey serum (Jackson ImmunoResearch Laboratories). An overnight incubation in a rabbit anti-c-fos (sc-52, lot \#F2510 Santa Cruz Biotechnology, CA, USA, diluted to 1:5000; sc-52, lot \#F3016 Santa Cruz Biotechnology, CA, USA, diluted to 1:500; or, ABE457, Millipore, Burlington, MA, USA, diluted to 1:2000) and rat anti-mCherry (M11240, Molecular Probes, Eugene, OR, USA, diluted to 1:100) primaries was followed by a biotinylated donkey anti-rabbit IgG secondary (1:1000, Jackson ImmunoResearch Laboratories) and Cy2-conjugated streptavidin (1:1000, Jackson ImmunoResearch Laboratories). Sections were observed under a fluorescence microscope to check for injection site accuracy and CNO-elicited activity.

\section{Data Analysis}

Pre-test and test data collection included the same trial specifications as trial training but used 3 bottles in a randomized, block design: 9 blocks in which each stimulus is randomly presented once. Collection of pre-test data excluded $\mathrm{CNO}$ administration. BW, licks to water/stimuli, lick latencies, and trial initiations were measured and plotted for graphical analysis. No further analysis of DREADDs data was conducted due to the inability to validate the effectiveness of the PBN-VTA circuit activation by CNO.

\section{Results 8.1}

Although activating this circuit was expected to affect feeding, specific effects were challenging to predict due to a paucity of congruent data available and lack of consensus in current publications. Although PBN projections to VTA are glutamatergic, we were uncertain to what extent this signaling influences DA versus GABA neurons in the VTA ${ }^{177,198}$. In fact, activating this circuit could have caused water, or even QHCl, to be consumed at levels similar to sucrose if it mimicked neuronal responses typically used to encode that a stimulus was palatable or caloric. Our model somewhat arbitrarily predicted consummatory and appetitive behavior to increase (based on the work of de Araujo and colleagues ${ }^{240,246}$ ).

Some cases were excluded due to one or more of the viral transfections being off target; however by and large the more pressing concern became the inability to validate activation of the circuit. The design included 3 microinjections total (both sides of the PBN and the midline VTA), which elevated the risk of exclusion (compared to fewer injections) for off-target injections. While the 4 cases observed had acceptable PBN injections, 2/4 were excluded due to off-target VTA injections. Moreover, a major 
setback occurred when PBN sections were observed and no FLI could be found in cells identified as having been isolated by the two viral transfections. Although the activation of PBN-to-VTA neurons could not be verified with immunohistochemistry, data from the experiment was graphed and observed (not shown). No obvious effects in licks or trial initiations to water, sucralose, or $\mathrm{QHCl}$ between groups were apparent, and data were not analyzed for statistical inferences. Instead, we opted to first measure the feeding effects of manipulating the VTA directly.

\section{Experiment 8.2}

\section{Animals}

23 mice were used for this VTA manipulation experiment (ages on surgery d: 45 $-82 \mathrm{~d}$, mean $=63 \pm 3 ; 14$ male, 10 female). 9 additional mice were excluded from the VTA manipulation experiment: 5 did not learn during training, 1 died after the taste stimulation data was collected, 1 showed no activity during testing, and 2 had misplaced cannula. Mice were assigned either to the drug group or the vehicle (control) group. Mice were tested in 3 iterations with varying number of mice per iteration. The test procedure was the same for all 3 iterations, except the first iteration received a higher $\mathrm{QHCl}$ concentration during the caloric test. The behavioral apparatus used was the same as that described in Experiment 8.1.

\section{Intra-VTA Guide Cannula Implantation}

Guide cannula were implanted in a separate cohort of mice to target the VTA for inhibition before measuring feeding. Mice were anesthetized with ketamine/xylazine and mounted on a stereotaxic frame. A small burr hole was drilled in the mouse's skull over the VTA (AP: -3.5, DV: 4.0, ML: 0.0), and a guide cannula (custom 4 mm C315G-SPC, PlasticsOne, Roanoke, VA, USA) was inserted. The guide cannula ended $2 \mathrm{~mm}$ dorsal to the intended target allowing this length to remain unaffected except by the injection cannula (custom C315I-SPC, PlasticsOne) which extended the remaining length when fully inserted. A screw (00-96 X 1/16, Invivo1, PlasticsOne) was placed near the cannula, and dental acrylic (resin liquid and powder, Lang Dental Manufacturing, Wheeling, IL, USA) was applied to the cannula and screw to fix the cannula in place.

\section{Training}

Mice were allowed to recover for at least two days after surgery before food and water restriction began for training (Table 8-2). High restriction of mice was based on BW: $0.5-2.5 \mathrm{~mL}$ of water was provided after a session (Table 8-3) only if the mouse licked at least 10 times and initiated at least 3 trials during the session (no lick count per trial requirements were made; no water was provided, if mice did not meet the 
Table 8-2. VTA inhibition train and test schedule.

\begin{tabular}{|c|c|c|c|c|}
\hline Phase $^{a}$ & Procedure $^{\mathrm{b}}$ & Restriction $^{\mathrm{c}}$ & Post-surgery Day $^{\mathrm{d}}$ & Contingency to Next Phase \\
\hline 0 & surgery/recovery & none & 0 & Survival of successful surgery \\
\hline 1 & $10 \mathrm{~s} \mathrm{~S}$ train & high & At least 2 & $\begin{array}{l}\text { At least } 2 \text { sessions in which at least } 3 \text { trials are initiated } \\
\text { and at least } 2 \text { trials include } 20 \text { or more licks (individual) }\end{array}$ \\
\hline 2 & "replete" \& $10 \mathrm{~s} \mathrm{~S}$ train & none & & $\begin{array}{l}\text { Body weight ratio average above } 1.01 \text { and at least } 2 \text { days } \\
\text { (all) }\end{array}$ \\
\hline 3 & $10 \mathrm{~s}$ WQS train & low & & 1 day \\
\hline 4 & $10 \mathrm{~s}$ WQS pre-test & low & & 1 day \\
\hline 5 & taste test & low & At least 8 & 1 day \\
\hline 6 & $10 \mathrm{~s} \mathrm{WQcSc}$ train & low & & 1 day \\
\hline 7 & $10 \mathrm{~s} \mathrm{WQcSc}$ pre-test & low & & 1 day \\
\hline 8 & calorie test & low & & 1 day \\
\hline
\end{tabular}

a. Mice remain at a behavioral phase until its contingency is passed. Phase 1 is passed individually; phase 2 requires all mice pass before phase 3 begins.

b. All behavioral procedures (i.e., procedure "10 s S train" and on) include $27-10 \mathrm{~s}$ trials with $120 \mathrm{~s}$ waits for first licks and $7.5 \mathrm{~s}$ IPIs. Procedure "10 s S train" uses 1 tube with sucralose. All procedures after use 3 tubes (water [W], bitter [Q], and sweet [S]). The session time minimum is $\sim 8 \mathrm{~m}$ and the maximum is $\sim 62 \mathrm{~m}$, which is within the period of drug effects. Beginning when all mice have entered phase 2, mice are held in the hand for 2 min prior to the session to habituate the mice to stay calm for the intraVTA injection and the stress before "hard data" collection. The airflow to prevent olfactory cues is also initiated when all mice have entered phase 2 to habituate them to the sound and feeling of the air. In "taste test" and "calorie test", muscimol is infused into the VTA. Calories are added by exchanging sucralose for sucrose and adding sucrose to a higher concentration of $\mathrm{QHCl}$.

c. Partial water and food high restriction: $0.5-2.5 \mathrm{~mL}$ of water is given only if the mouse licks at least 10 times and initiates at least 3 trials; $0.5-2.5 \mathrm{~g}$ of food is given based on BW (chart below); if BW drops below 75\% without passing to next phase, the mouse is excluded and "repleted". Partial water\&food low restriction: $1-3 \mathrm{~mL}$ of water is given daily after session based on BW (chart below); $1-3 \mathrm{~g}$ of food is given based on BW (chart below); if BW drops below 85\%, the water and food given are doubled.

d. All surgeries are completed the first day of the experiment. All mice are given at least 2 days to recover from the surgery before training begins. All mice are allowed at least 8 days before testing begins. 
Table 8-3. VTA inhibition water and food partial restriction schedules.

\begin{tabular}{lcc}
\hline & \multicolumn{2}{c}{ Water or Food $(\mathbf{m L}$ or g) } \\
\cline { 2 - 3 } Training Day 1 BW $(\mathbf{g})$ & High & Low \\
\hline $10-14.9$ & 0.5 & 1.0 \\
$15-19.9$ & 1.0 & 1.5 \\
$20-24.9$ & 1.5 & 2.0 \\
$25-29.9$ & 2.0 & 2.5 \\
$30-34.9$ & 2.5 & 3.0 \\
\hline
\end{tabular}


requirements); $0.5-2.5 \mathrm{~g}$ of chow was given daily regardless of session behavior (Table 8-3); if BW dropped below 75\% without the mouse passing to the next phase, it was excluded and "repleted". A lower restriction, also based on BW, was used while test data was collected: $1-3 \mathrm{~mL}$ of water and $1-3 \mathrm{~g}$ of chow was given daily after the session regardless of session behavior (Table 8-3); if BW dropped below $85 \%$, the water and food given were doubled. This type of combined restriction (i.e., high and low) is common in taste experiments; animals are motivated by appetite to sample palatable stimuli in a concentration-dependent manner, while they are not as strongly fluid restricted. A high level of fluid-only restriction (i.e. little or no daily supplemental water) tends to result in mice licking any neutral-to-palatable fluid at a maximal rate, causing a loss of sensitivity in palatability and appetite measurements. Thus, higher restriction works well to accelerate training, but lick data collection may benefit from lower restriction. While using this procedure, percentage of initial BW, which may be used as a rough estimate of appetite, indicated these intentions were achieved (Figure 8-2). BW\% dropped sharply during training, and after a shallower drop, it remained relatively stable during testing.

Table 8-2 provides a detailed explanation of the training and testing schedule used for this experiment, including each phase's procedures, (water/food) restrictions, start relative to post-surgical day, and contingencies placed on mouse behavior for passing to the next phase. The first two phases varied in the number of days depending on how quickly mice learned to lick $3 \mathrm{mM}$ sucralose from the sipper tube in the rig. To pass the first phase, mice had to accumulate at least 2 sessions ( 1 session per day) in which they initiated at least 3 trials and in at least 2 of those an accumulation of at least 20 licks. Mice passed phase 1 individually. The second phase allowed mice to become replete after the previous phase's high restriction and before the low restriction of the subsequent phases. This second phase lasted at least 2 days after the last mouse completed phase 1 and required the cohort to maintain a BW ratio average of at least 1.01 . The mice passed phase 2 as a cohort. The additional phases were 1 day each, of which 3 were training. Phase 3 initiated the low restriction. Neophobia can occur in mice wherein novel taste stimuli, regardless of palatability, are consumed less avidly to prevent accidental poisoning (see Learning in Feeding and Synaptic Adaptation section in Chapter 9 for more on neophobia). Prior exposure before testing allows mice to habituate and prevents collection of aberrant test data. Phases 3 and 6 gave mice prior exposure to taste stimuli to overcome neophobia before test data was collected in phases $4,5,7$, and 8 .

\section{Muscimol}

Muscimol was prepared from a stock solution kept at $10 \mathrm{~g} / \mathrm{L}$ (M1523-5MG, Sigma-Aldrich) by mixing $20 \mu \mathrm{L}$ of stock into $480 \mu \mathrm{L}$ of $0.9 \%$ sterile saline for a final concentration of $0.4 \mathrm{~g} / \mathrm{L}$. Both the stock and test concentrations were kept at $4{ }^{\circ} \mathrm{C}$ until testing. Mice in the drug group were microinjected into the VTA with $0.3 \mu \mathrm{L}$ of muscimol solution at a constant rate over $2 \mathrm{~min}$, and the injection cannula was left in place for an additional 2 min before placing the mouse in the rig for testing. The vehicle group received intra-VTA saline only. 


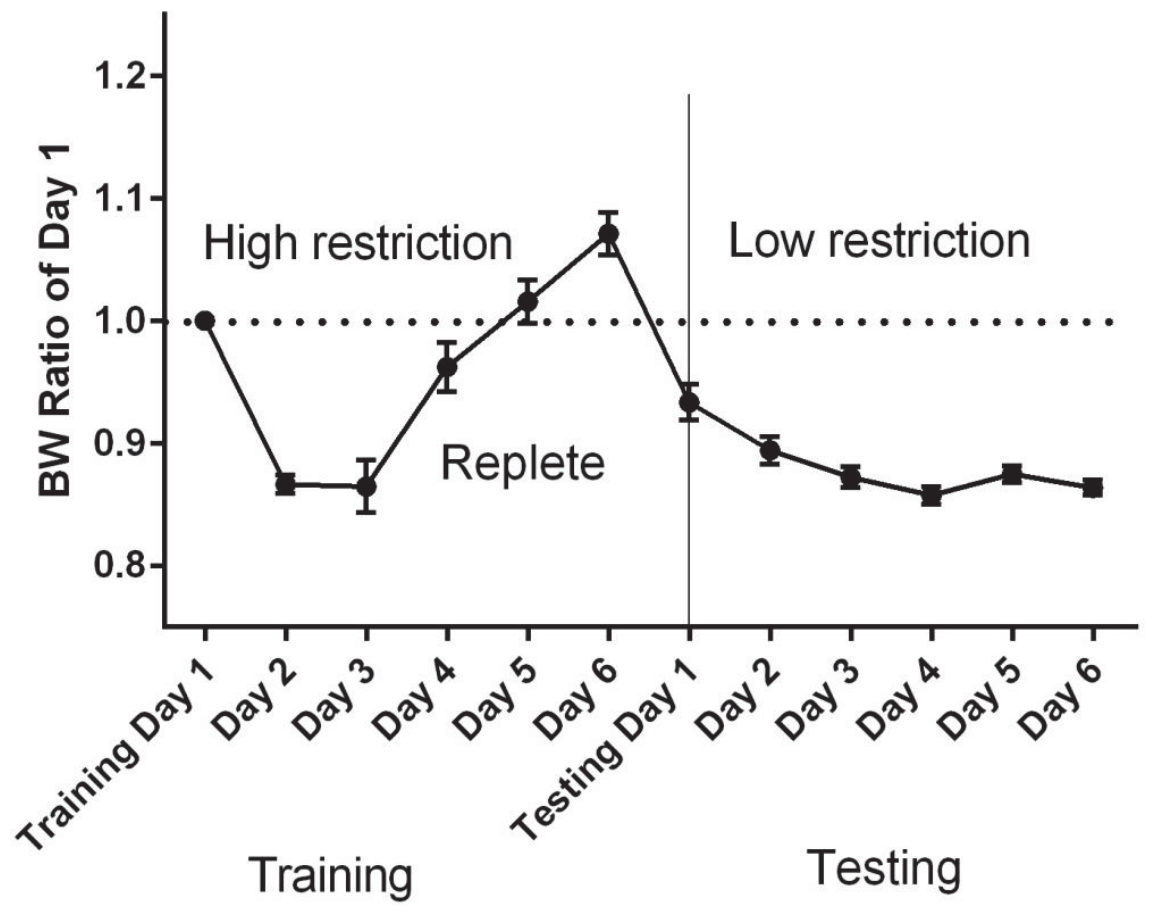

Figure 8-2. Body weight (BW) fluctuation throughout experiment.

BW remained relatively stable throughout the experiment as compared to the Day 1 measurement taken (dotted horizontal line). BW dropped sharply upon initiation of the high restriction schedule and gradually increased as mice passed training and became replete. The drop in BW was shallower going into testing (solid vertical line) and remained relatively constant during the low deprivation schedule. 


\section{Taste Solutions}

All tastants were prepared fresh daily using reagent grade chemicals (SigmaAldrich) dissolved in distilled water. Calorie-free taste stimuli were tested first to prevent metabolic effects from interfering with outcome measures: $0.1 \mathrm{mM} \mathrm{QHCl}$ and $3 \mathrm{mM}$ sucralose. Caloric stimuli were tested second; to test for palatability and aversiveness effects while keeping in calories constant sucrose concentration was used for both palatable and aversive stimuli but it was adulterated with $\mathrm{QHCl}$ to make it aversive: 0.3 M sucrose, 0.3 M sucrose + 0.3 mM QHCl.

\section{Histology}

Mice were anesthetized prior to intra-VTA microinjection of Chicago sky blue (Sigma-Aldrich) and perfusion. Brains were collected and sectioned for histological verification of cannula placements (Figure 8-3).

\section{Results 8.2}

\section{Non-caloric Taste Test}

All mice (groups combined) reliably licked the three stimuli (3 mM sucralose, 0.1 $\mathrm{mM}$ QHCl, and water) throughout the 27 trials (9 trials/stimulus) in the pretest session. Total consumption during this session was 408.5 licks (range $=149-833$ ), although mice consumed the sucralose much more avidly (mean licks $=341.4 ; 82 \%$ of the total) than either $\mathrm{QHCl}$ (mean licks $=38.64 ; 11 \%$ of the total) or water $($ mean licks $=28.5 ; 7 \%$ of the total). These percentages did not differ between groups, although lick totals did. Total consumption per session (sum of licks to all stimuli combined) was examined between groups during both the pretest and (drug) test session using a 2-way ANOVA (between-subjects factor for group $\mathrm{X}$ within-subjects factor for session). There was a significant effect of group $\left(\mathrm{F}_{(1,20)}=20.85 ; \mathrm{p}<0.001\right)$ but not session; however, there was a significant group $X$ session interaction $\left(F_{(1,20)}=12.47 ; p=0.002\right)$. This interaction effect seemed to indicate a drug-dependent effect on total consumption, with a decrease only evident in the mice receiving muscimol. However, post-hoc comparisons showed significant group differences $(\mathrm{p}<0.05)$ in licking during both pre-test and test session, although the mean group difference was much larger on the test day (421.5 licks) than on the pretest day (239.0 licks).

Further analysis showed total licks were highly correlated between both days in the 11 mice receiving vehicle $(\mathrm{r}=0.86, \mathrm{p}<0.001)$, but not in the drug group. In other words, although consumption in the vehicle group increased slightly from pretest to test session, individuals behaved consistently regardless of whether they had relatively low or high lick counts (e.g., range for this group was 149 - 833 licks in the pretest session). On the other hand, the majority of mice in the drug group (8 of 11) decreased total 


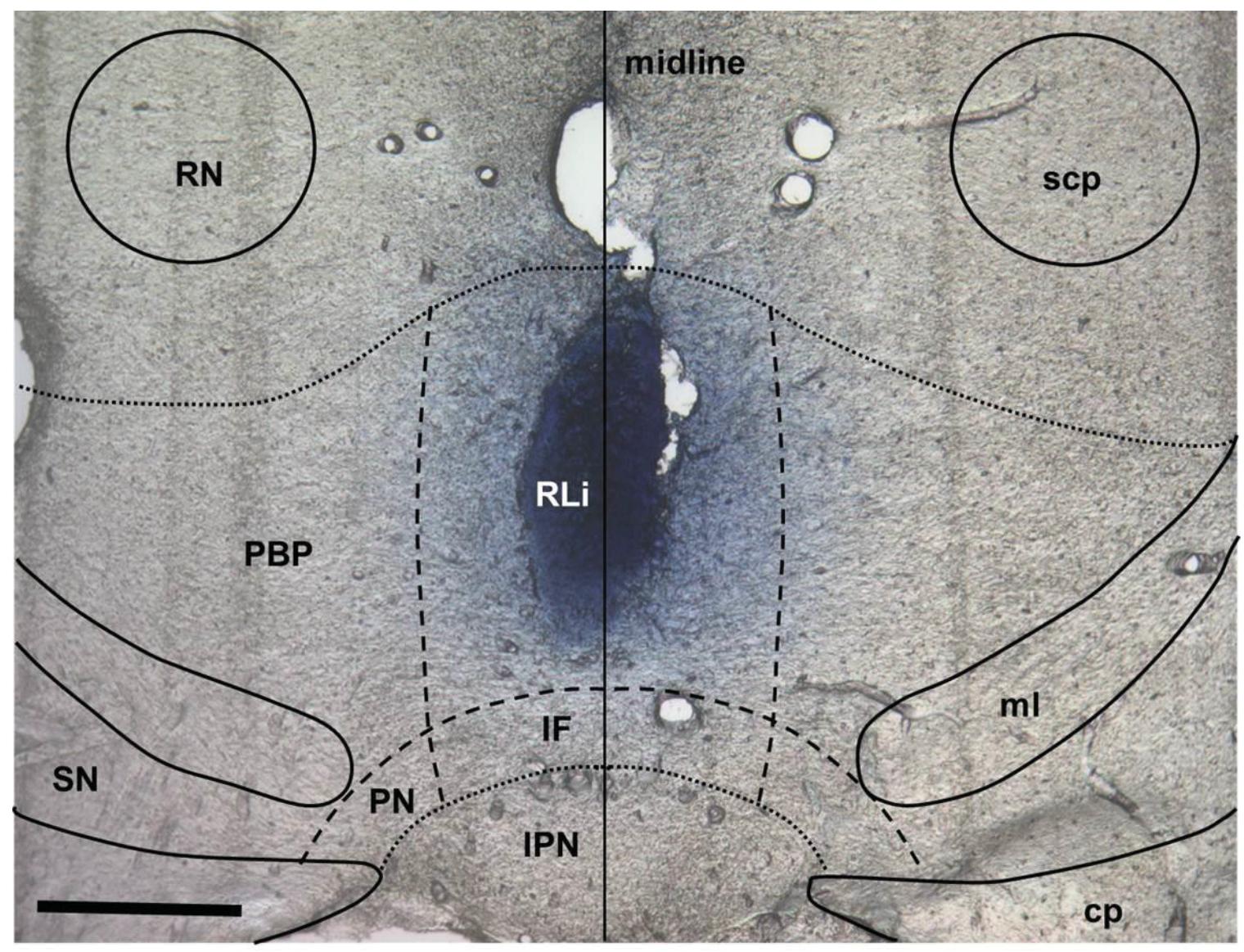

Figure 8-3. Exemplar of VTA cannula placement indicated by injections.

After testing, Chicago Sky blue was injected via guide cannula to check for correct placement. Most cannula were within the acceptable anterior-posterior limits of the VTA, and therefore, muscimol was considered to have targeted the desired location. Only 2 out of 25 cannula missed the VTA. Note that the smaller volume of dye $(0.1 \mu \mathrm{L})$ used was not expected to fully recapitulate the diffusion of muscimol, but only to provide an estimation of the point from which the drug was infused; the full $0.3 \mu \mathrm{L}$ infusion of muscimol likely diffused throughout the entire VTA. PBP, pigmented parabrachial; RLi, rostral linear nucleus of the raphe; IF, interfascicular; PN, paranigral; cp, cerebral peduncle; IPN, interpeduncular nucleus; ml, medial lemniscus; RN, red nucleus; SN, substantia nigra. Scale bar $=500 \mu \mathrm{m}$. 
consumption on the test day, presumably due to an inhibitory effect of the muscimol. The cause of individual and group differences among B6 mice in our experiment during the pretest (where we did not expect to see group differences, as no drug was administered) was not immediately clear, and may have been coincidental. Total licks were not explained by variation in age, BW, or sex (correlation analyses; ns). Of course, phenotypic variation among even highly isogenic individuals (i.e., within inbred strains) has been found in just about every type of behavior, including consumption (e.g. Wahlsten et al. $2003^{289}$; Loos et al. $2015^{290}$ ).

To account for individual baseline differences in licking (and thereby correct for group differences), potential drug effects on the individual taste stimuli were examined by computing a pre-test/test ratio (mean of total licks on test day / mean of total licks on pretest day) for each mouse. This data is shown in Figure 8-4. Ratio data were logtransformed to correct for non-normality and analyzed with a 2-way ANOVA (group X stimulus). Only the interaction between these variables was found to be significant $\left(\mathrm{F}_{(2,59)}\right.$ $=3.59 ; \mathrm{p}=0.038)$. Post-hoc tests (Bonferroni) showed that the groups significantly differed $(\mathrm{p}<0.05)$ in pre-test/test ratio for sucralose, but not $\mathrm{QHCl}$ or water, with drug mice showing reduced licking (mean ratio $=0.73$ ) relative to vehicle mice $($ mean ratio $=$ 1.30; Figure 8-4A). In general, this reduced licking was spread out evenly across the test session-i.e., mid-session onset of satiety did not appear to play a role in attenuation of licking. One way to quantify this is by comparing the number of trials initiated to each stimulus. Vehicle mice essentially licked sucralose in all possible trials (of which there are 9$)$ during both pretest $($ mean $=8.82$ trials) and test (mean $=8.91)$ sessions, whereas drug-treated mice showed, on average, a slight decrease in trials initiated on the test day (8.73 to 7). However, these differences were not quite significant for sucralose, or for the other stimuli, as assessed via non-parametric tests. A second way to assess whether decreases in licking are spread evenly across the test session is to visualize cumulative lick functions for each group on each day (Figure 8-5). For mice receiving vehicle, functions are linear for sucralose in both sessions, indicating consistent sampling behavior across the 9 trials. The drug group also has a linear function on the pre-test day, but deviates somewhat from this on the test day, reflecting the tendency of some of the mice in this group to terminate licking behavior to sucralose earlier in the session. Notably, however, the functions for both groups for $\mathrm{QHCl}$ on both days are similar, again underlining the specificity of the drug effect on sucralose. Water licking was not examined in this fashion due to it being the least avidly consumed of the three stimuli.

\section{Caloric Taste Test}

We next examined total consumption in the sessions with caloric stimuli $(0.3 \mathrm{M}$ sucrose, $0.3 \mathrm{M}$ sucrose $+0.3 \mathrm{mM} \mathrm{QHCl}[\mathrm{S}+\mathrm{Q}]$, water). Among all mice, total consumption in the pre-test averaged 640.9 licks; $61 \%$ of these were to sucrose, $37 \%$ to the sucrose adulterated with $\mathrm{QHCl}$, and just $2 \%$ to water. As before, these percentages

did not vary among group, but total licks did. There was both a significant effect of group $\left(F_{(1,14)}=8.82 ; p=0.01\right)$, as well as a group $X$ session interaction $\left(F_{(1,14)}=6.75 ; p=0.02\right)$ on total licks across the pretest and test sessions. As was the case with the non-caloric 

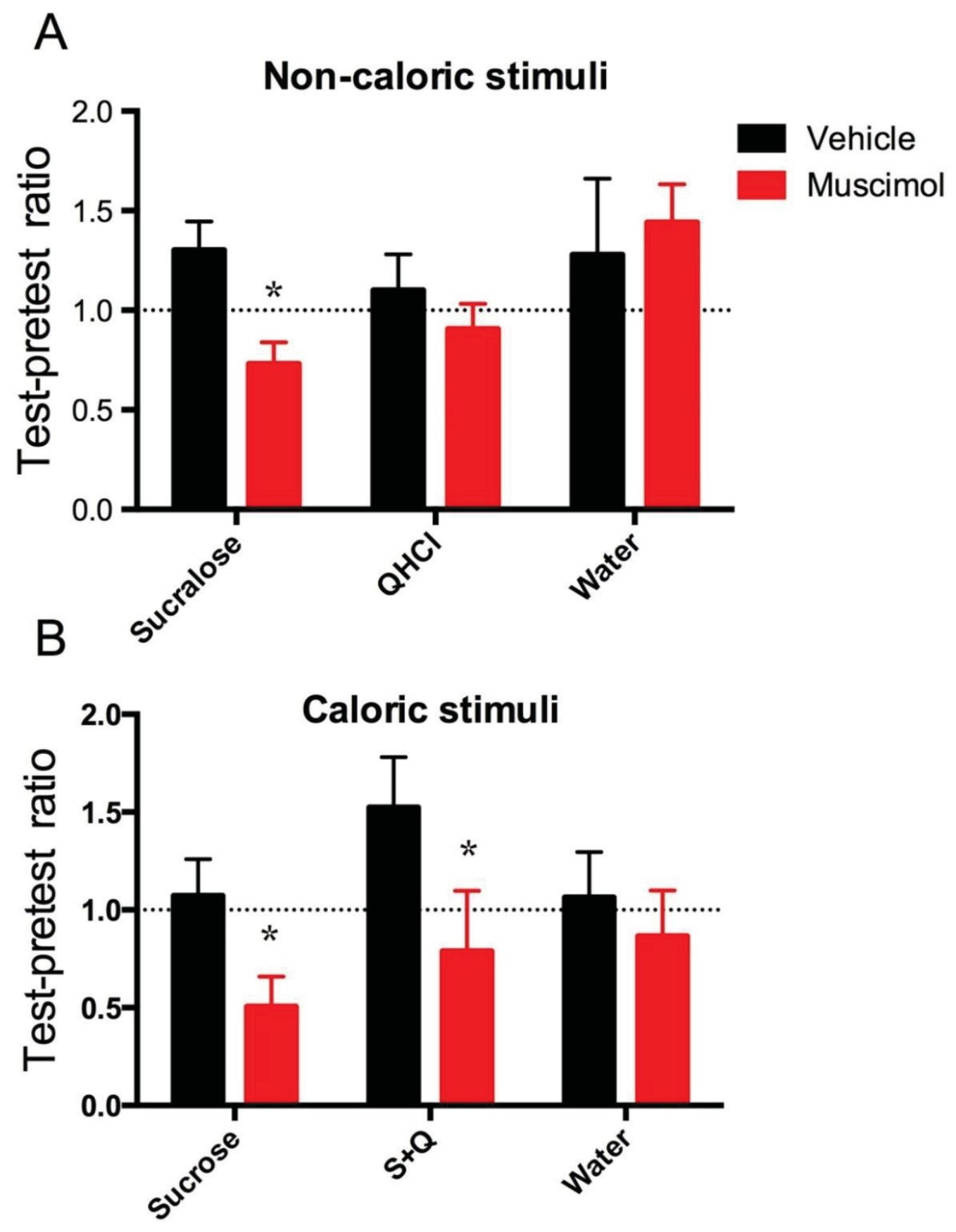

\section{Stimulus}

Figure 8-4. Test-pretest ratios to non-caloric and caloric taste stimuli.

Mice that received intra-VTA muscimol injections consumed less palatable and caloric and caloric stimuli relative to controls which received saline. No effect was seen to aversive, non-caloric $\mathrm{QHCl}$ or neutral, non-caloric water. Data shown was logtransformed for analysis (see text). Asterisks denote significant effects $(\mathrm{p}<0.05)$. 


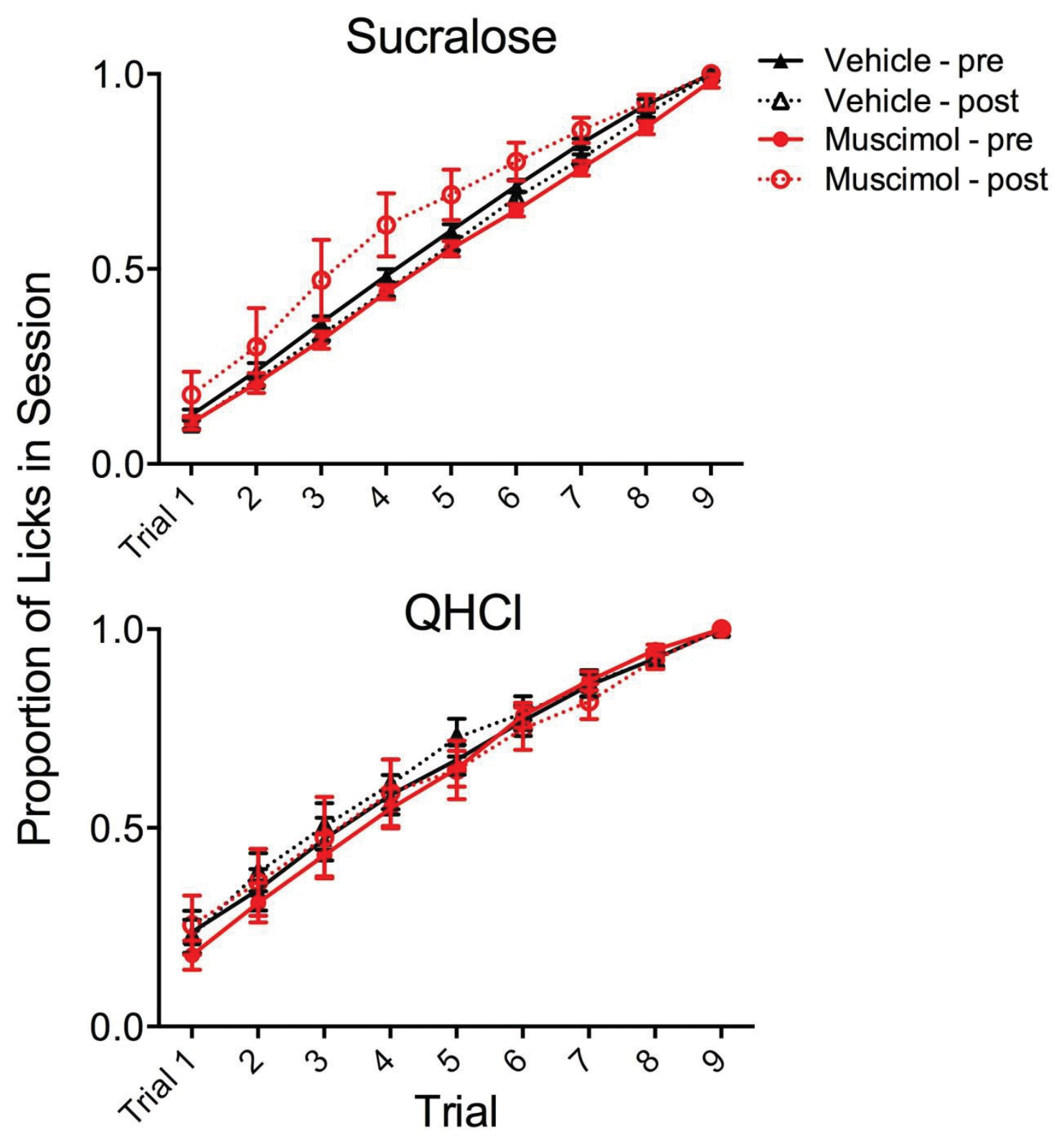

Figure 8-5. Cumulative licking in sessions to non-caloric taste stimuli.

Cumulative licking was used as one method to assess effects on appetite. Consumption of stimuli during the non-caloric trials was relatively linear and unaffected from pre-test to test conditions or by intra-VTA muscimol injections, indicating no effects on appetite. 
stimuli, vehicle mice licked more than drug mice in both sessions, with this group difference being greater on the test day (mean difference $=660.6$ licks) than on the pretest day (mean difference $=329.4$ licks). Again, within the vehicle group, both "high" and "low" consumers were relatively consistent with their behavior from one session to the next $(\mathrm{r}=0.87 ; \mathrm{p}=0.005)$, whereas $7 / 8$ mice in the drug group decreased consumption on the test day $(\mathrm{r}=0.29 ; \mathrm{ns})$.

When pretest/test ratios (log transformed) for each stimulus were compared (Figure 8-4B), a significant effect was found for group $\left(F_{(2,40)}=13.92 ; p<0.001\right)$ but not stimulus. Post-hoc tests confirmed that the ratios were reduced for both sucrose and $\mathrm{S}+\mathrm{Q}(\mathrm{p}<0.05)$, but not water, indicating a decrease in licking of these stimuli relative to baseline resulting from muscimol infusion. In addition, drug-treated mice initiated significantly fewer trials than vehicle mice to both sucrose (mean \# trials $=6$ vs. $9 ; \mathrm{p}=$ $0.002)$ and $\mathrm{S}+\mathrm{Q}(6$ vs. $9 ; \mathrm{p}=0.01)$. When cumulative licking of either stimulus was visualized (Figure 8-6), the drug group showed a marked deviation from linearity on the test day; these functions show that these mice generally ceased licking behavior to both stimuli earlier in the session than they did in the pre-test. Vehicle mice possessed linear functions for both sessions, maintaining their consumption of these stimuli at a near constant rate. Unlike the data collected with non-caloric stimuli, these data suggest that muscimol not only affected avidity for caloric stimuli within trials, but that it also affected satiety, causing mice to limit their overall intake.

\section{Conclusions}

Although our methods were unable to isolate the PBN-to-VTA circuit, we did show that both taste and caloric information must be processed in the VTA. Boekhoudt et al. $2017^{241}$ found, somewhat unexpectedly, that activating the VTA-to-NAc circuit decreased food intake. One might have hypothesized that NAc DA levels would have increased from activating this circuit, leading to increased consumption of food.

However, the food may be neutral or only slightly palatable unlike a sucrose pellet or solution. Increased consumption with VTA-to-NAc activation may require the meal be palatable. Further, their manipulation did increase feeding frequency, suggesting the urge to consume had been increased. Hence, if our method worked, we might infer from their results that the PBN innervates cells in the VTA other than the DAergic projections to the NAc. Otherwise decreased consumption of water and increased trial initiations might have occurred. In contrast, no effects were found when we tried to specifically activate the PBN-to-VTA circuit using virally-delivered CRE-dependent DREADDs. However, it remains possible our methods did not successfully activate this circuit. Therefore, it is possible that our data show that this circuit has no appreciable effect on feeding, but further investigation is certainly needed to confirm this. Our experiment was discontinued because FLI was not found in cells which appeared to be positive for DREADDs. Thus, we were unable to validate PBN-to-VTA activation was occurring. Although other means were possible to verify circuit activation (e.g., electrophysiology), this was not an option for us at the time and the experiment became unfeasible. This prompted us to move on to 


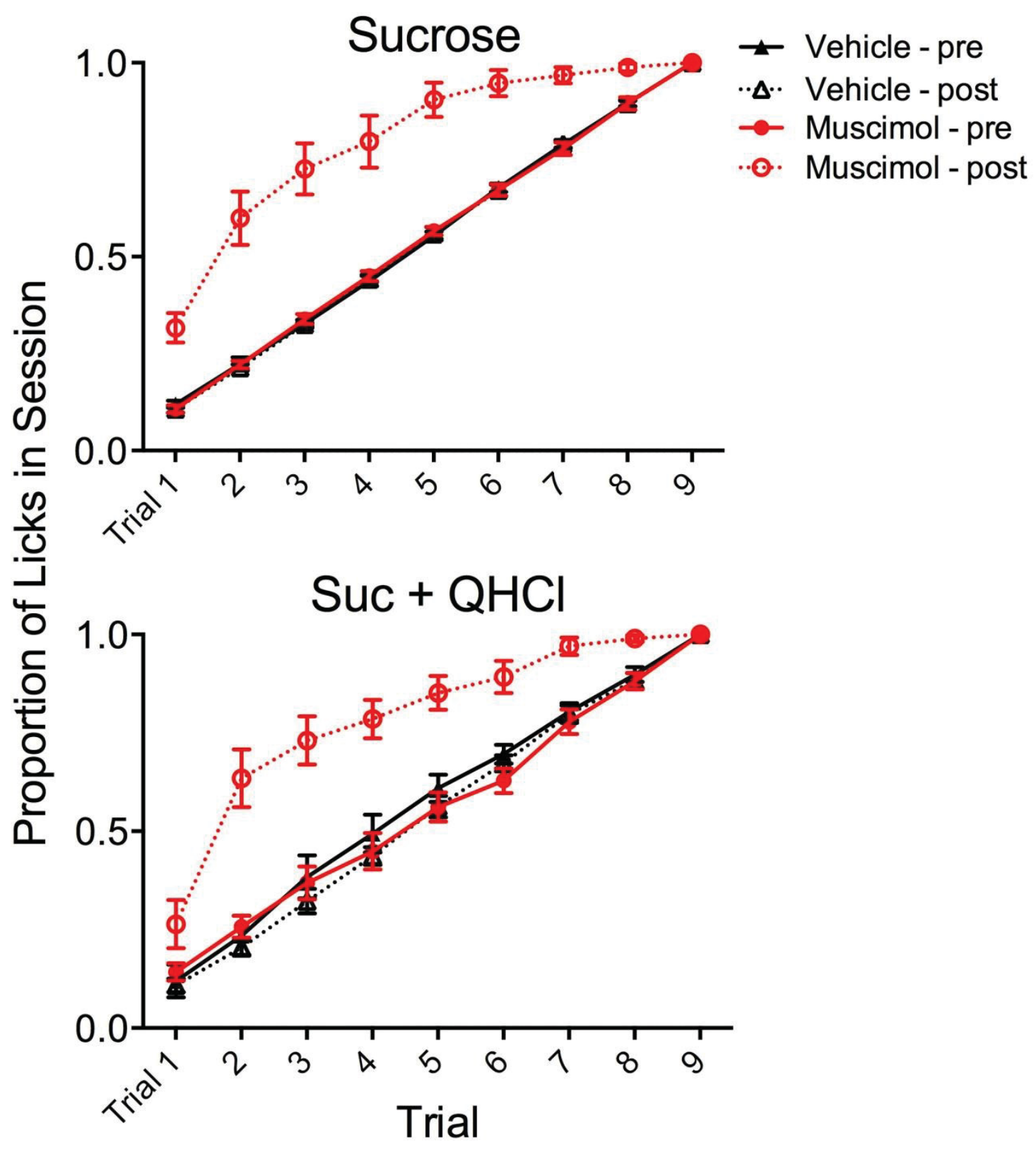

Figure 8-6. Cumulative licking in sessions to caloric taste stimuli.

Consumption of caloric stimuli during the caloric trials was relatively linear and unaffected from pre-test to test conditions in control mice. Mice that received intra-VTA muscimol injections showed a marked deviation in their consumption of caloric stimuli during testing (from both controls and their pre-test behavior), indicating the onset of satiety had occurred sooner as a result of the GABA agonist. 
a different and more direct experimental option-i.e., inhibiting the VTA with intracranial muscimol and gauging the effects on taste and consummatory behavior.

The group of mice with muscimol-inhibited VTAs decreased consumption compared to their own pre-test measurements and to a control group of mice that received saline on test day. The control group's behavior remained consistent from pre-test and test day, supporting our inference that the VTA manipulation decreased consumption. Muscimol-inhibited-VTA mice decreased consumption of sucralose, but not $\mathrm{QHCl}$ or water, suggesting this effect is taste-specific (in this case, for a palatable stimulus as opposed to aversive or neutral). However, consumption of both a palatable and aversive tasting stimulus decreased when it also contained calories. It is also likely the mixture of sucrose and QHCl, while not as appetitive as sucrose, was also not as repulsive as an imaginary "caloric version of QHCl” could have been. Although test-pre-test ratio decreases to caloric stimuli were like those to non-caloric sucralose, an apparent calorieinduced satiety effect was caused by inhibiting the VTA. Consumption of both sucrose and $\mathrm{S}+\mathrm{Q}$ decreased more rapidly (i.e., less consumption in later trials) in mice that received muscimol on test day, presumably an effect of hastening satiety onset. IntraVTA muscimol also notably affected locomotion which became erratic or slowed; however, the lack of a difference in water consumption in the drug group means the locomotor effects were not a factor influencing our interpretation of altered response to taste and caloric stimuli.

Our results contrast with other studies in which intra-VTA muscimol caused increased feeding ${ }^{251,252}$. One possible reason our results might differ could be a differential effect in the VTA based on the location of injections (see Beier et al., $2015^{202}$ for VTA to different outputs). Echo et al. (2002) ${ }^{251}$ and Khaimova et al. (2004) ${ }^{252}$ did intra-VTA injections bilaterally which may have inhibited more lateral aspects of the VTA than our midline injection. Shimura et al. (2002) ${ }^{211}$, who also found increased feeding after injecting the benzodiazepine agonist, midazolam, did so systemically with i.p. administration. Thus, our results here may confirm that manipulating VTA neurons in different subnuclei has different effects on behavior. Additional experiments could test this by using small volumes of drug and injecting into slightly different VTA locations while measuring consumption behaviors. A second difference between the Echo et al. and Khaimova et al. studies and ours is the palatability/appetitiveness of the stimulus. The cited studies used rat chow to measure feeding which might at best be considered slightly palatable. Further, no alternatives were offered to the rats meaning the effects on stimuli with varying palatability were not measured. Our method allowed us to simultaneously assess the effects of inhibiting the VTA on consumption of aversive, appetitive, and neutral stimuli.

The lowered consumption of caloric stimuli on test day could raise a question about the interpretation of responses to calories themselves. How can we have a caloric effect if the mice are not ingesting caloric stimuli? Possible explanations include 1) caloric content can be sensed in the oral cavity and does not actually require ingestion, and 2) mice responded to stimuli in accordance to the memory of "caloric" taste. Although artificial sweeteners and caloric sugars both taste sweet, it is possible that some 
means of differentiating noncaloric from caloric stimuli exist at the level of the oral cavity. This may occur via the "taste" transduction mechanisms themselves (followed by differential caloric sensation but similar taste sensation) or this could occur through a completely separate transduction mechanism. Further investigation is necessary to determine the process mediating this effect. It is also possible the mice formed a memory after the sucrose-calories pairing experienced in the first two sessions. The decreased consumption would have been due to a response to the taste cue signaling a caloric stimulus. In this case, the behavioral response is still in part due to the caloric content of the stimulus but less directly. Finally, it should be reiterated that the responses were shown to be due to the stimuli themselves because the response to water was unaffected. 


\section{CHAPTER 9. DISCUSSION}

The experiments conducted for this dissertation collectively present further investigation into the connectivity between the PBN and VTA and the possible role of this taste/reward interface in feeding. Although a selective manipulation of the PBN-toVTA circuit could not be satisfactorily achieved, both the projection's existence and the ability of the VTA to alter feeding were supported. This investigation elucidates one example of a direct connection between canonical taste and reward nuclei. Together with other taste/reward interfaces, these connections likely help produce the complex and highly-regulated feeding behaviors that both prevent us from accidental poisonings and urge us to consume to remain nutritionally replete.

We quantified the amount of neuronal activity (FLI+ cells) in the PBN in response to taste stimuli with varying palatability similar to previous studies ${ }^{59,66,67}$. These studies showed subnuclei specific effects to taste stimuli. For example, Yamamoto et al. (1994) ${ }^{66}$ used similar DAB staining in rats for FLI and found caudal el and em activity to aversive bitter stimuli and $\mathrm{dl}$ and cl activity to palatable. Tokita and Boughter (2016) ${ }^{45}$ recently characterized the $\mathrm{PBN}$ with electrophysiology, finding roughly topographic and somatotopic patterns in taste-responsiveness, some of which were specific to subnuclei. Sucrose-best neurons were found in higher numbers in BC and QHCl-best in lateral subnuclei. Our results agree to an extent with these previous studies and also suggest the dependence of FLI on taste transduction via the TRPM5 channel. We found an elevation in FLI to QHCl in the el, an aversive bitter stimulus, in agreement with the Yamamoto et al. finding. This was corroborated when we compared the FLI response to QHCl with the response to water; further, we showed $\mathrm{KO}$ mice had a diminished FLI response to $\mathrm{QHCl}$ in the el. These results also agreed with the general finding that QHCl-best neurons were typically found laterally ${ }^{45}$. It was interesting that sucrose also elicited a significant FLI response in the el of $\mathrm{KO}$ mice compared with responses to water or QHCl. However, the response was comparable to the elevation seen in $\mathrm{B} 6$ mice. Together, these results confirm the finding from Palmiter and colleagues (Carter et al., $2013^{73}$ ). The FLI in the el was also higher in more visceral, rostral sections of the PBN (data not shown). Regardless, this effect to sucrose was more likely post-ingestive as this subnucleus has been implicated for in other studies ${ }^{73}$. Indeed, Palmiter and colleagues have repeatedly found a subset of CGRP+ neurons which project to the CeAlc from the PBN and both inhibit feeding (induce satiety) and are capable of recapitulating a CTA like that caused by pairing i.p. $\mathrm{LiCl}$ with a taste stimulus ${ }^{72}$. $\mathrm{PBN}$ neurons in the el may respond primarily to aversive and repulsive stimuli, and by relaying information about such perceptions to the CeAlc, participate in a role to decrease consumption of possibly toxic substances. With evidence that the CeA also projects to the VTA, it is possible this el-CeAlc circuit further influences the VTA and DA release as well. This would likely impact the incentive salience of associated stimuli possibly via a learning mechanism (see below).

The sparse overlap between FG and CTb labeling in the PBN suggested these pathways are mostly discrete. Unlike the findings for the previously recognized thalamic and ventral pathways, in which more overlap was found ${ }^{59,69}$, the projections to the VTA 
and VPMpc barely collateralize at all. In fact, the two projections mostly originated from opposite sides of the brain with VTA projecting from the dm, m, and vl, while the VPMpc from the $\mathrm{m}$, wa, and el. If the PBN is indeed sending taste quality or hedonic information to the VTA, future studies will need to account for this in their design to effectively test the hypothesis. These findings also contrast with some previously found when retrograde tracing identified mostly ipsilateral PBN projections to the VTA ${ }^{80}$. This previous study also found labeling in all PBN subnuclei; however, VTA projection cells tended to be more laterally located than in our study. This discrepancy may indicate an anatomical difference between mice and rats or some variation specific to the B6 strain. These mostly separate pathways to the VTA and VPMpc also suggest that any taste or visceral information being relayed could be target-specific. For example, the VPMpc may play a major role in taste quality recognition and discrimination, whereas the pathway to the VTA may function more for taste and visceral reward. Placed in the context of other models of reward circuits, the PBN-to-VTA communication may provide influence in assessments of stimulus salience and promote urges to continue or discontinue consumption of sapid stimuli.

FLI in the retrograde tracer study in response to sucrose and $\mathrm{QHCl}$ was elevated as compared to water. Further, some differential activity in individual subnuclei was found including in the dm, m, el, cl, and dl. As in Yamamoto et al. (1994) ${ }^{66}$, we found the $\mathrm{cl}$ and $\mathrm{dl}$ to be responsive to palatable stimuli. Unfortunately, less could be concluded from the results of quantifying double labeled cells. Although the pathways to the VTA and VPMpc were discrete, the FLI in projection cells may be stochastic with respect to stimulus, i.e., the amount of double-labeling merely correlated with overall FLI. Nonetheless, it is worth simultaneously examining the results of tracing and FLI to see what the data suggest about how the PBN subnuclei inform downstream targets about taste stimuli. In this case, the various differences in how the PBN projects to VTA and VPMpc and in how activity is elicited to stimuli of varying palatability might suggest the contralateral $\mathrm{m} \mathrm{PBN}$ is most capable of communicating the salience of sucrose to the VTA while contralateral dm may transmit information about aversive QHCl. Meanwhile, the VPMpc appears to receive input from ipsilateral $\mathrm{m}$ and el to communicate about palatable sucrose or aversive QHCl.

$\mathrm{TH}+$ and GAD67+ cells were identified throughout the VTA, including in specific subnuclei. Unsurprisingly, the TH labeling was useful in identifying this nucleus and its subnuclei. Our delineations easily followed from other publications identifying the localization of these subnuclei (e.g., Oades and Halliday, $1987^{156}$; Lammel et al., 2008 ${ }^{94}$ ), including the RMTg ${ }^{198,201}$. Others have observed the VTA might co-express DA and GABA (e.g., Gonzalez-Hernandez et al., $2001^{291}$; Olson and Nestler, $2007^{183}$; NairRoberts et al., $2008^{201}$ ), but our study suggests that the small percentage of cells found in these studies may in fact be due to the inadequate methods they used. Nair-Roberts et al. critiqued of their study, "the cell labeling methods [we] used...are not suitable for an accurate quantitative analysis of DL neurons as both the in situ and immunohistochemical reactions produced opaque precipitates that interfered with the detection of the other. An accurate analysis of the possible colocalization of $\mathrm{TH}$ [and] GAD...will require the use of different cell labeling methods that utilize different markers e.g. fluorescence...labeling" 
(Nair-Roberts et al., $2008^{201}$ ). Indeed, our study took advantage of two methods which fluorescently labeled VTA neurons, and in conjunction with high-resolution confocal microscopy, provided detailed data about the 3-dimensional presence of $\mathrm{TH}$ - and GAD67-expressing cells, of which none were DL. This finding supports the conclusion that DA and GABA neurons compose entirely separate cell populations in the VTA. The RMTg was identified with GAD67+ cells and seen to cross from a relatively lateral position to medial as it was traced posteriorly. Like the subnuclei identified with $\mathrm{TH}$, the localization of the RMTg was in agreement with other publications ${ }^{198}$.

Surprisingly, no significant differences of FLI to tastants were found when we stained the VTA with DAB, even in comparisons of B6 and KO mice. This contrasts with studies in which DAB was used to identify increases in response to food in rats ${ }^{212}$. This might reflect a low sample size $(n=6)$, however it was similar to that of other studies which specifically found elevated FLI expression in response to palatable meals in rats 212. Thus, our results seem to suggest it may in fact be the case that the VTAs of mice are not so sensitive to effects of tastes with varying palatability. In contrast, when measuring FLI with fluorescent IHC, FLI in a posterior region of the VTA was significantly higher to $\mathrm{QHCl}$ than water or sucrose. With respect to FLI, the RLi and CLi had significant responses to $\mathrm{QHCl}$ compared to water; with respect to $\mathrm{TH}, \mathrm{CLi}$ had significant differences in water, sucrose, and $\mathrm{QHCl}$. The $\mathrm{RLi}$ is present at the AP level at which the heightened response to $\mathrm{QHCl}$ was found. The $\mathrm{CLi}$ is present more posteriorly, but the CLi data agree when adjusted by both TH and FLI. The short span in the AP dimension of VTA in which this effect was found may explain why it was apparently missed when DAB IHC was used. That is, the one section sampled (tVTA, $-3.88 \mathrm{~mm}$ post bregma) that overlapped in these two experiments happened to not contain a significantly higher level of FLI, but this may be less conclusive if this study missed the relatively few sections in which $\mathrm{QHCl}$ did elicit more FLI. Another possible explanation is that one or more outlier measurements occurred in which the sections were shifted posteriorly as compared to those measured for FLI to sucrose and water. This seems unlikely for two reasons: 1) the effect was found in two consecutive sections, meaning the shift had to be greater than 160 $\mu \mathrm{m}$, and 2) the delineations used throughout the VTA sections were standardized and consistently applied, meaning the shift would have caused highly noticeable alterations in the anatomy present. In fact, one case, in which the anatomy was noticeably different, actually seemed to shorten rather than extend this region. Although this case was forced into the standardized delineations mentioned, examination of the data indicates its unusual anatomy had little effect when it was averaged across cases. We conclude a posterior portion of the VTA, likely in the area of the $\mathrm{RLi} / \mathrm{CLi}$ is responsive to taste stimuli, especially when they are aversive.

Some interesting considerations present when we compare the findings from the experiments described in Chapters 6 and 7. In the DAB and fluorescent cases in these studies, both the PBN and the VTA had higher FLI measured using DAB as compared to the fluorescent IHC methods. This may reflect a methodological limitation of DAB immunohistochemistry, if only the highest intensity of FLI was seen when visualized with fluorescence. This finding suggests an important consideration to make when comparing the significant results to taste stimuli in the PBN and VTA. For example, one 
disparity occurs in the effects seen in the cl of the PBN, in which sucrose caused a higher FLI response than water. It is interesting that no el PBN projection occurs to the VTA, yet the VTA seems most responsive to the QHCl taste stimulus. Perhaps the $\mathrm{QHCl} /$ aversive taste information is reaching the VTA through other pathways, but it is also possible the PBN is using the projection from the $\mathrm{dm}$ to send this information. As mentioned, an additional cross-cutting analysis of data from Experiments 6.1 and 7.1 was conducted to help explain the discord between the PBN and VTA FLI results when using DAB. To examine this, we calculated the correlations of the FLI data between the PBN and VTA. The sucrose and $\mathrm{QHCl}$ were correlated, but water was not $(\mathrm{r}=0.7,0.81$, and 0.32 , respectively). This suggested the VTA responded to stimuli other than taste or had a "noisier" baseline. Although our methods aimed to control for such a possibility (e.g., comparing tastes to water, comparing wild-type to putatively taste-blind mice), methods which better compensate for the noise might produce different results.

Inhibiting the VTA with muscimol decreased the consumption of palatable, appetitive, and caloric stimuli. Further, it may have shortened the onset of satiety of caloric stimuli. Although little effect was found in the VTA to essentially the same stimuli (actually of higher concentrations) in FLI experiments, these behavioral results suggest the VTA is necessary for increasing the consumption of foods when they are palatable and/or nutritious (i.e., high in calories). The shortened onset of satiety to caloric stimuli may reflect a default behavior which occurs when the VTA does not send signals that the stimulus is caloric. Interestingly, a study in which the VTA was injected with muscimol or the $\mathrm{GABA}_{\mathrm{B}}$ agonist, baclofen, rats increased their consumption of food ${ }^{251}$. A few methodological differences that may explain the difference between this and our study are the use of rats instead of mice, the provision of essentially palatably-neutral rat chow versus more palatable taste solutions in our study, and a shorter access period to the stimulus being used for the consumption measurement ( $4 \mathrm{~h}$ vs $\sim 30 \mathrm{~min}$, respectively). Our methods of injecting the VTA also differed, a factor which likely makes a difference in behavioral outcome as was shown in Beier et al. (2015) ${ }^{202}$. As opposed to our midline injections, Echo et al. (2002) ${ }^{251}$ and Khaimova et al. (2004) ${ }^{252}$ did intra-VTA injections bilaterally which likely inhibited lateral aspects more effectively. Indeed, our TH + FLI results support this in that midline nuclei, the CLi and RLi, were the two with significant differences. Shimura et al. (2002) ${ }^{211}$, who also found increased feeding after injecting the benzodiazepine agonist, midazolam, did so systemically with i.p. administration. These findings strongly suggest VTA subnuclei differ in responsiveness to taste and caloric stimuli and can cause different behaviors based on which are excited/inhibited (i.e., medial vs lateral).

Our findings in context with the ongoing research in the field help fill in the gap in understanding the network mediating feeding that exists between the peripheral inputs and forebrain. The release of DA in the ventral striatum to sucrose in trpm 5 -/- mice was shown to be due to caloric post-ingestive feedback (sucralose was used as a control) ${ }^{246}$. Later, neurons in the insular cortex (IC) was suggested to code post-ingestive information about sucrose ${ }^{292}$, since IC-lesioned trpm5 -/- mice do not develop preferences for sucrose. "Dorsal IC is not necessary for the detection of the postingestive value of sucrose" (Oliveira-Maia et al., $2012^{292}$ ). The differences were explained in their 
consumption measurements based on when the measurement was made: preconditioning, conditioning, or post-conditioning. Lesioned mice, like sham mice, drank more sucrose than water in the conditioning sessions indicating they were detecting the post-ingestive value of sucrose. Since lesioned mice did not make the association of sipper tube position with post-ingestive effects of sucrose, dorsal IC must be necessary for this function. Moreover, separate circuits at levels higher than the PBN and VTA were implicated in the communication of hedonic and nutritional information about sugar 240. Stimulating D1R expressing ventral striatum (VS) neurons increased sucralose intake but not when it is adulterated with bitter denatonium. Stimulating D1R expressing dorsal striatum (DS) neurons increased sucralose intake, even when it is adulterated. Stimulating D2R expressing neurons had little effect. Activating the VS projection to the VP increased sweetener intake, and activating the VP blocked this effect, confirming the VSVP projection is GABAergic. Activating the DS projection to the substantia nigra pars reticulata (SNpr) also increases sweetener intake, which is not blocked by VP activation, but is by SNpr activation. The principle investigator of these studies, Ivan de Araujo, makes the argument that reward of foods is not from taste, but from post-ingestive effects. He concludes that sweetness is neither necessary nor sufficient for formation of memories of energy sources ${ }^{293}$. However, the DA system and its mesoaccumbens and nigrostriatal pathways have been shown to be connected to centers for both taste and feeding; modulation of DA release likely drives urges to consume palatable food and to approach its associated cues. Further, while visceral/caloric input may explain long term feeding behavior, it cannot explain the short term indulgence in palatable foods to which subjects are naïve. Our findings provide evidence of a parallel and proximal pathway which may contribute to these short term increases in consumption when novel, palatable stimuli are presented to rodents.

\section{Study Limitations}

The inherent limitation to the use of the expression of the IEG $c F o s$ as an indicator of neuronal activity is well known ${ }^{294}$. FLI can be found in neurons that are not found to be activated using electrophysiological measurements. Conversely, it has also been shown that c-Fos expression underestimates the actual number of neurons activated ${ }^{294,295}$. Given these limitations, one may wonder why bother to use such a method. In response, we argue that, like other activity proxies (e.g., calcium imaging, oxygen in fMRI), FLI correlates to neuronal activation more often than not. We accept this limitation and agree our measurements are approximations of activation. In fact, i.o. stimulation with citrate elicits Fos expression in the same forebrain areas that PET identified as active ${ }^{296}$. More importantly, the expression of c-Fos itself indicates a biological event has occurred within the cell regardless of whether it fired an action potential. Therefore, investigating the neurobiological correlates of this cellular event may prove to be useful in and of itself. Finally, FLI measurement has the advantage over electrophysiology of providing accurate localization of the activity, meaning the two techniques complement one another. 
Another limitation specific to this investigation, as opposed to the field at large, was the inability to validate the activation of PBN neurons transfected with DREADDs. Unfortunately, this failure may reflect our reliance on elevated FLI as the measure chosen for validation. This is, however, considered an acceptable means of validation. It is possible that electrophysiological recordings in the PBN may have revealed activation of neurons after CNO administration. Most regrettably, the negative results found when ostensibly activating the PBN-to-VTA circuit remain inconclusive as a direct consequence of this limitation.

\section{Future Directions}

\section{A Feeding CPG}

Additional work will be necessary to help explain how the PBN and VTA fit into the larger network of nuclei which control feeding. One nucleus long known to be strongly implicated in the control of feeding is the LH. Takashi Yamamoto and Tsuyoshi Shimura (2008) ${ }^{57}$ indicate the $\mathrm{LH}$ as the endpoint for converging circuits modulating feeding ${ }^{297}$. Anand and Brobeck (1951) ${ }^{298}$ found that bilateral destruction of the LH in rats caused complete cessation of feeding. Later, Delgado and Anand (1953) 299 stimulated the LH in cats and saw a $1000 \%$ increase in food intake. Taken together, these results suggest the LH is both necessary and sufficient in causing feeding. Importantly, the $\mathrm{LH}$ and other areas also interact with the areas for affect to influence feeding. For example, $\mathrm{OX}$ and $\mathrm{MCH}$ neurons project to NAc, VTA, LC, hippocampus, thalamus, AMY, cortex, and various nuclei of the hypothalamus ${ }^{222-224}$. Activating LH neurons with OX increased reinstatement of reward seeking. Similar results were found when it was injected directly into the VTA ${ }^{300}$. LH-projecting PBN neurons show increased activity to i.o. gustatory stimuli ${ }^{59}$. The lateral habenula has inhibitory control over the mesolimbic DA system and forms reciprocal connections with the LH and VTA ${ }^{212,301-303}$. Thus, taking the LH as a "center" for feeding, one could work backward to construct a network for feeding that includes taste and reward nuclei such as the PBN and VTA.

Ultimately, the neurobiology of feeding must prime or cause the motor behavior that produces these processes through central pattern generators (see Selverston, 2010 for a review of $\mathrm{CPGs}^{304}$ ), but many investigators point out multiple neural origins motivate behavior, including some with higher cognitive functions. Some feeding behaviors with evidence for CPGs include licking, masticating, and swallowing. Basal ganglia and substantia nigra pars compacta (SNpc) are involved with serial grooming behavior and may also be related to facial expressions used to measure hedonic impact in taste reactivity experiments. These findings fit in with a general theory that striatal neurons are part of a system mediating the "syntax of sequential motor behavior (action syntax)" 305 . GC neural states and their transitions were identified (with Hidden Markov Modeling [see Miller and Katz, $2010^{306}$ ]), which predict expel/swallow decisions from electromyocardiograms by $<500 \mathrm{~ms}{ }^{307}$. This state follows an identification state in the 
ensemble. Future studies could work to discover the roles the PBN and VTA play in these overall network behaviors which affect feeding.

Results of experiments in which the VTA is unilaterally manipulated may indicate there is a crossing over in the transmission from the VTA to areas more directly responsible for feeding, but our finding that the PBN projections to the VTA are contralateral could also feasibly influence this. When one side of the VTA of rats was lesioned and the contralateral side stimulated, the increase in Fos expression was amplified compared to the increase seen in the unlesioned but unilaterally stimulated group ${ }^{162}$. Similarly, a shortened latency to eat in the unlesioned, unilaterally stimulated group was amplified in the group with a contralateral VTA lesion. Unilaterally lesioned rats that received no VTA stimulation showed no changes in Fos or behavior. This indicated the lesions had suppressed the inhibition of afferent brain areas to the VTA ${ }^{162}$. Further, unilateral block of excitatory input via NMDA receptors and current into the contralateral VTA led to a shortened feeding latency ${ }^{254}$, suggesting at least the feeding effect is mediated by glutamate. Taken with our finding that the PBN-to-VTA circuit connects contralaterally, the effects of unilateral manipulations could work in part due to descending feedback that then crosses over from the pons to the midbrain.

Two distinct anatomical DA subpopulations in the VTA, especially between medial and lateral, may reflect differential functions for various behaviors, including feeding. Caudal and rostral IVTA neurons project to the lateral NAcs ${ }^{83}$. Dorsolateral VTA neurons project to lateral NAcs or dorsal striatum ${ }^{96}$. Caudal mVTA neurons project to the BLA, as well as the medial NAcs, NAcc, and PFC ${ }^{83,96}$. Further, a major distinction of inputs to the VTA can be made in terms of where the receiving VTA neuron projects. For example, VTA neurons receiving excitatory glutamatergic and cholinergic input from LDTg inputs project to the NAcs ${ }^{143,177}$, whereas VTA neurons receiving inhibitory GABAergic input from the RMTg project to the NAcc and PFC 143,177,308. Additional work is needed to determine what, if any, differential these projection patterns contribute to feeding behaviors.

\section{Learning in Feeding and Synaptic Adaptation}

Three types of taste learning are attenuation of neophobia, CTA, and conditioned taste preference ${ }^{57,309}$. Animals are initially conservative about how much of a novel tasting stimulus they will consume, hence the term "neophobia." It is thought this behavior reduces any deleterious effects of a possibly toxic, unknown substance. After exposure to the substance and pending agreeable post-ingestive effects, the animal will consume the substance more readily. This is known as attenuation of neophobia. CTA, originally discovered by John Garcia (1955) ${ }^{310}$, occurs when a novel substance is consumed that evokes gastrointestinal malaise ${ }^{43,311,312}$ After which, the animal avoids the consumption of that substance and others that taste similar. Conditioned taste preference may occur if an animal associates positive experiences after ingesting something with an inherently neutral or aversive taste ${ }^{313-316}$. 
Activation of the el-CeAlc projection also induces CTA ${ }^{72}$. Evidence from CTA studies suggest the displeasure of taste may be mediated via the CeA and PBN. Despite the work by Richard Palmiter and colleagues, Yamamoto and Shimura suggest the BLA may be more important than the CeA in acquisition and retention of CTA $57,66,297,317$. However, lesioning the BLA may cause a loss of neophobia rather than loss of CTA ${ }^{43}$. Lesioning the CeA, but not the BLA prevents CTA development ${ }^{318}$. Still, it is known that the $\mathrm{PBN}$ projects to the BLA, possibly giving this nucleus an indirect role in CTA. Lesions of the PBN prevents the acquisition of CTA, predicted to be due to a reduced or abolished perception of illness US ${ }^{43,319-322}$. Lesioning of the $\mathrm{m}$ is assumed to prevent integration of the neural representations of the US and CS because none of the other four stages of CTA development are disrupted ${ }^{43,321,323}$. Since neurotoxic DA lesions leave evidence that mechanisms for CTA development remain intact ${ }^{17,144}$, phasic DA responses may not be necessary for displeasure to taste to occur. Instead, recent work suggests displeasure of taste may be mediated via GABA. Systemic baclofen, a GABA agonist, had no effect on the expression of conditioned flavor preference, but reduced its acquisition ${ }^{324}$. It also enhanced the acquisition of conditioned flavor aversion.

Synaptic adaptations in VTA neurons accompany functional and behavioral changes seen with regards to DA release and reward-seeking and could play an important role in learning about taste and food stimuli and their cues. Depending on whether the stimulus is stress, drugs, or some other motivational stimulus, DA release is evoked in anatomically and functionally different DA cell subpopulations. Such variation in synaptic adaptations are suggested by differences Maria De Luca and colleagues have found in forebrain DA release to food versus drugs ${ }^{239}$. Habituation of DA release to food rewards occurs in the NAcs, but not in $\mathrm{mPFC}$ and NAcc. Chronic, but not acute morphine, can sensitize the NAcs so that habituation of DA release to food reward no longer occurs. This may mean that although food itself cannot cause "food addiction" via sensitizing the DA system, the prior use of some addictive substances can. Synaptic adaptations are largely known to be mediated via glutamatergic input. Activating postsynaptic NMDA receptors leads to bursts of action potentials in VTA DA neurons ${ }^{83,325}$. Synaptic adaptations in the VTA are well-known to be affected by various addictive drugs ${ }^{326}$. Among the most important neural circuit modifications that contribute to development of addiction are changes in the properties of excitatory synapses on midbrain VTA DA neurons ${ }^{83,326}$. For example, drugs of abuse induce potentiation of $\alpha-$ amino-3-hydroxy-5-methyl-4-isoxazoleproprionic acid (AMPA) receptor-mediated synaptic transmission in VTA DA neurons ${ }^{83}$. Various LTDs and LTPs are produced in VTA. Since the PBN projection to the VTA is glutamatergic, it contributes an excitatory potential and could have strong learning effects up to those resembling addiction to food taste. At the least, it seems likely that synaptic adaptations in the VTA affect the changes in DA release De Luca found and could in turn mediate learning long-term, taste-feeding contingencies.

Additional functional anatomical investigation has begun to tease apart the interaction of the DA system with the two hedonic dimensions of affect (i.e., pleasure and displeasure) ${ }^{177}$. The circuits from the laterodorsal tegmentum to DAergic VTA to NAcs are for reward (e.g., activation mediated conditioned place preference [CPP]). The 
circuits from the lateral habenula to DAergic VTA to mPFC are for aversion (e.g., activation mediated conditioned place aversion [CPA]). Therefore, it seems plausible these two sets of circuits could play similar roles in the development of conditioned preferences and aversions of tastes, respectively. The lateral habenula also forms GABAergic projections to the RMTg. D1 antagonists to the MPFC prevented CPA development. D1 and D2 antagonists to the lateral NAcs prevented CPP development. This suggests a plausible neurobiological substrate for how the mesocorticolimbic DA system could produce urge generally, leaving the determination of behavioral directionality to the involvement of polarized systems. Another mechanism at work for the interaction between displeasure and urge may be GABA agonists or glutamate antagonists in the caudal NAcs leading to inhibition of GABAergic medium spiny neurons which disinhibit neurons in LH, VP, or VTA ${ }^{210,327-333}$. Future work should attempt to determine what roles these circuits for affect play in development of taste aversions and preferences.

\section{Concluding Remarks}

Overall, this project has confirmed that both the PBN and VTA function to communicate taste and reward information. Although its function remained elusive, the evidence of the direct path from the PBN to the VTA was fortified. Further, to our knowledge, this was the first time evidence has been found of its existence as a mostly separate pathway from the thalamic pathway. Combined with the knowledge of this circuit, the activity in these nuclei and the ability to affect consumption by inhibiting the VTA suggest the PBN and VTA work together to influence feeding by detecting and integrating information about palatability and calories. Future work will be able to identify the precise functions of this circuit as well as those of the more indirect connections of the PBN to the VTA.

We have made strides since Pavlov and Thorndike began explaining conditioning, processes that include some we use to learn to consume savory and satiating foods. After Olds and Milner came Schultz, then Berridge, further enlightening us to the basic neural mechanisms underlying our tendency to reward ourselves. We can now thank Lammel, De Luca, de Araujo, Palmiter, and others for continuing to elucidate the network underlying taste-mediated feeding, including how it responds to stimuli from either end of the valence spectrum. Finally, the evidence discovered from experiments described in this dissertation added a few more dots which may be connected to the theoretical whole that explains how an electrically-connected, cellular network generates the complex output of self-nourishment. 


\section{LIST OF REFERENCES}

1. Finger S. Origins of neuroscience : a history of explorations into brain function. New York: Oxford University Press; 1994.

2. $\quad$ Chaudhari N, Roper SD. The cell biology of taste. J Cell Biol 2010; 190(3): 28596.

3. Liman ER, Zhang YV, Montell C. Peripheral coding of taste. Neuron 2014; 81(5): 984-1000.

4. Smith DV, Boughter J. Taste: Vertebrate Central Pathways; 2009.

5. Loney GC, Blonde GD, Eckel LA, Spector AC. Determinants of taste preference and acceptability: quality versus hedonics. J Neurosci 2012; 32(29): 10086-92.

6. Aristotle. De anima. 1994.

7. Bartoshuk LM. The psychophysics of taste. Am J Clin Nutr 1978; 31(6): 1068-77.

8. Ikeda K. On a new seasoning. J Chem Soc Tokyo 1909; 30: 820-36.

9. Spence C. Multisensory Flavor Perception. Cell 2015; 161(1): 24-35.

10. Berridge KC. The debate over dopamine's role in reward: the case for incentive salience. Psychopharmacology (Berl) 2007; 191(3): 391-431.

11. Marks LE. A Brief History of Sensation and Reward. In: Gottfried JA, ed. Neurobiology of Sensation and Reward. Boca Raton (FL); 2011.

12. Cabanac M. Physiological role of pleasure. Science 1971; 173(4002): 1103-7.

13. Pavlov PI. Conditioned reflexes: An investigation of the physiological activity of the cerebral cortex. Ann Neurosci 2010; 17(3): 136-41.

14. Berridge KC. From prediction error to incentive salience: mesolimbic computation of reward motivation. Eur J Neurosci 2012; 35(7): 1124-43.

15. Your Amazing Brain. www.youramazingbrain.org (accessed Jan 2018.

16. Schultz W. Predictive reward signal of dopamine neurons. J Neurophysiol 1998; 80(1): 1-27. 
17. Berridge $\mathrm{KC}$, Robinson TE. What is the role of dopamine in reward: hedonic impact, reward learning, or incentive salience? Brain Res Brain Res Rev 1998; 28(3): 309-69.

18. Henderson LW, Knight T. Integrating the hedonic and eudaimonic perspectives to more comprehensively understand wellbeing and pathways to wellbeing. International Journal of Wellbeing 2012; 2(3): 196-221.

19. Delle Fave A, Brdar I, Freire T, Vella-Brodrick D, Wissing MP. The eudaimonic and hedonic components of happiness: Qualitative and quantitative findings. Soc Indic Res 2011; 100: 185-207.

20. Frankl VE. Man's search for meaning. Boston: Beacon Press; 2006.

21. Nichols DE. Hallucinogens. Pharmacol Ther 2004; 101(2): 131-81.

22. Gresch PJ, Strickland LV, Sanders-Bush E. Lysergic acid diethylamide-induced Fos expression in rat brain: role of serotonin-2A receptors. Neuroscience 2002; 114(3): 707-13.

23. Gresch PJ, Smith RL, Barrett RJ, Sanders-Bush E. Behavioral tolerance to lysergic acid diethylamide is associated with reduced serotonin-2A receptor signaling in rat cortex. Neuropsychopharmacology 2005; 30(9): 1693-702.

24. Gresch PJ, Barrett RJ, Sanders-Bush E, Smith RL. 5-Hydroxytryptamine (serotonin)2A receptors in rat anterior cingulate cortex mediate the discriminative stimulus properties of d-lysergic acid diethylamide. J Pharmacol Exp Ther 2007; 320(2): 662-9.

25. Arango V, Underwood MD, Mann JJ. Serotonin brain circuits involved in major depression and suicide. Prog Brain Res 2002; 136: 443-53.

26. McKim WA. Drugs and behavior: An introduction to behavioral pharmacology. Upper Saddle River (NJ): Pearson Prentice Hall; 2007.

27. Huxley A. The doors of perception. New York City: HarperCollins; 1954.

28. Barnes NM, Sharp T. A review of central 5-HT receptors and their function. Neuropharmacology 1999; 38(8): 1083-152.

29. Fuster JM. The prefrontal cortex. Cambridge (MA): Academic Press; 2008.

30. Kandel ER. Disorders of mood: Depression, mania, and anxiety disorders. Principles of Neural Science. Amsterdam: Elsevier; 2000. 
31. Wikisource. https://en.wikisource.org/wiki/Ethics_Spinoza)/Part 3 (accessed Dec 2017.

32. Papanicolaou A. 2013.

33. Berridge KC, Ho CY, Richard JM, DiFeliceantonio AG. The tempted brain eats: pleasure and desire circuits in obesity and eating disorders. Brain Res 2010; 1350: 43-64.

34. Grigson PS. Like drugs for chocolate: separate rewards modulated by common mechanisms? Physiol Behav 2002; 76(3): 389-95.

35. Levine AS, Billington CJ. Opioids as agents of reward-related feeding: a consideration of the evidence. Physiol Behav 2004; 82(1): 57-61.

36. Cooper SJ, Higgs S. Neuropharmacology of appetite and taste preferences. In: Legg CR, Booth DA, eds. Appetite: neural and behavioural bases. New York City: Oxford University Press; 1994: 212-42.

37. Dickinson A, Balleine B. The role of learning in the operation of motivational systems. In: Gallistel CR, ed. Stevens' handbook of experimental psychology: learning, motivation, and emotion. New York City: Wiley and Sons; 2002: 497534.

38. Kelley AE, Baldo BA, Pratt WE, Will MJ. Corticostriatal-hypothalamic circuitry and food motivation: Integration of energy, action and reward. Physiol Behav 2005; 86: 773-95.

39. Barraco R, el-Ridi M, Ergene E, Parizon M, Bradley D. An atlas of the rat subpostremal nucleus tractus solitarius. Brain Res Bull 1992; 29(6): 703-65.

40. Contreras RJ, Beckstead RM, Norgren R. The central projections of the trigeminal, facial, glossopharyngeal and vagus nerves: an autoradiographic study in the rat. J Auton Nerv Syst 1982; 6(3): 303-22.

41. Halsell CB, Travers JB, Travers SP. Gustatory and tactile stimulation of the posterior tongue activate overlapping but distinctive regions within the nucleus of the solitary tract. Brain Res 1993; 632(1-2): 161-73.

42. Whitehead MC, Frank ME. Anatomy of the gustatory system in the hamster: central projections of the chorda tympani and the lingual nerve. J Comp Neurol 1983; 220(4): 378-95.

43. Reilly S, Bornovalova MA. Conditioned taste aversion and amygdala lesions in the rat: a critical review. Neurosci Biobehav Rev 2005; 29(7): 1067-88. 
44. Hamilton RB, Norgren R. Central projections of gustatory nerves in the rat. $J$ Comp Neurol 1984; 222(4): 560-77.

45. Tokita K, Boughter JD, Jr. Topographic organizations of taste-responsive neurons in the parabrachial nucleus of C57BL/6J mice: An electrophysiological mapping study. Neuroscience 2016; 316: 151-66.

46. Lundy RF, Jr., Norgren R. Gustatory system. In: Paxinos G, ed. The rat nervous system. San Diego (CA): Elsevier Academic Press; 2014: 733-60.

47. Whitehead MC. Gustatory. In: Watson C, Paxinos G, Puelles L, eds. The mouse nervous system. San Diego (CA): Elsevier Academic Press; 2012: 571-88.

48. Tokita K, Inoue T, Boughter JD, Jr. Afferent connections of the parabrachial nucleus in C57BL/6J mice. Neuroscience 2009; 161(2): 475-88.

49. Tokita K, Boughter JD, Jr. Sweet-bitter and umami-bitter taste interactions in single parabrachial neurons in C57BL/6J mice. J Neurophysiol 2012; 108(8): 2179-90.

50. Tokita K, Yamamoto T, Boughter JD, Jr. Gustatory neural responses to umami stimuli in the parabrachial nucleus of C57BL/6J mice. J Neurophysiol 2012; 107(6): 1545-55.

51. Gill CF, Madden JM, Roberts BP, Evans LD, King MS. A subpopulation of neurons in the rat rostral nucleus of the solitary tract that project to the parabrachial nucleus express glutamate-like immunoreactivity. Brain Res 1999; 821(2): 251-62.

52. Herbert H, Moga MM, Saper CB. Connections of the parabrachial nucleus with the nucleus of the solitary tract and the medullary reticular formation in the rat. $J$ Comp Neurol 1990; 293(4): 540-80.

53. Norgren R, Leonard CM. Taste pathways in rat brainstem. Science 1971; 173(4002): 1136-9.

54. Norgren R, Leonard CM. Ascending central gustatory pathways. J Comp Neurol 1973; 150(2): 217-37.

55. Travers JB. Efferent projections from the anterior nucleus of the solitary tract of the hamster. Brain Res 1988; 457(1): 1-11.

56. Williams JB, Murphy DM, Reynolds KE, Welch SJ, King MS. Demonstration of a bilateral projection from the rostral nucleus of the solitary tract to the medial parabrachial nucleus in rat. Brain Res 1996; 737(1-2): 231-7. 
57. Yamamoto T, Shimura T. 4.24 - Roles of Taste in Feeding and Reward A2 Masland, Richard H. In: Albright TD, Albright TD, Masland RH, et al., eds. The Senses: A Comprehensive Reference. New York: Academic Press; 2008: 437-58.

58. Rolls ET. Smell, taste, texture, and temperature multimodal representations in the brain, and their relevance to the control of appetite. Nutr Rev 2004; 62(11 Pt 2): S193-204; discussion S24-41.

59. Tokita K, Armstrong WE, St John SJ, Boughter JD, Jr. Activation of lateral hypothalamus-projecting parabrachial neurons by intraorally delivered gustatory stimuli. Front Neural Circuits 2014; 8: 86.

60. Bernard JF, Alden M, Besson JM. The organization of the efferent projections from the pontine parabrachial area to the amygdaloid complex: a Phaseolus vulgaris leucoagglutinin (PHA-L) study in the rat. J Comp Neurol 1993; 329(2): 201-29.

61. Halsell CB. Organization of parabrachial nucleus efferents to the thalamus and amygdala in the golden hamster. J Comp Neurol 1992; 317(1): 57-78.

62. Karimnamazi H, Travers JB. Differential projections from gustatory responsive regions of the parabrachial nucleus to the medulla and forebrain. Brain Res 1998; 813(2): 283-302.

63. Norgren R. Taste pathways to hypothalamus and amygdala. J Comp Neurol 1976; 166(1): 17-30.

64. Veening JG. Subcortical afferents of the amygdaloid complex in the rat: an HRP study. Neurosci Lett 1978; 8(3): 197-202.

65. Yokota S, Kaur S, VanderHorst VG, Saper CB, Chamberlin NL. Respiratoryrelated outputs of glutamatergic, hypercapnia-responsive parabrachial neurons in mice. J Comp Neurol 2015; 523: 907-20.

66. Yamamoto T, Shimura T, Sakai N, Ozaki N. Representation of hedonics and quality of taste stimuli in the parabrachial nucleus of the rat. Physiol Behav 1994; 56(6): 1197-202.

67. Yamamoto T, Sawa K. Comparison of c-fos-like immunoreactivity in the brainstem following intraoral and intragastric infusions of chemical solutions in rats. Brain Res 2000; 866(1-2): 144-51.

68. Yamamoto T, Sawa K. c-Fos-like immunoreactivity in the brainstem following gastric loads of various chemical solutions in rats. Brain Res 2000; 866(1-2): 13543. 
69. Tokita K, Inoue T, Boughter JD, Jr. Subnuclear organization of parabrachial efferents to the thalamus, amygdala and lateral hypothalamus in C57BL/6J mice: a quantitative retrograde double labeling study. Neuroscience 2010; 171(1): 35165.

70. Fulwiler CE, Saper CB. Subnuclear organization of the efferent connections of the parabrachial nucleus in the rat. Brain Res 1984; 319(3): 229-59.

71. Alden M, Besson JM, Bernard JF. Organization of the efferent projections from the pontine parabrachial area to the bed nucleus of the stria terminalis and neighboring regions: a PHA-L study in the rat. J Comp Neurol 1994; 341(3): 289314.

72. Carter ME, Han S, Palmiter RD. Parabrachial calcitonin gene-related peptide neurons mediate conditioned taste aversion. $J$ Neurosci 2015; 35(11): 4582-6.

73. Carter ME, Soden ME, Zweifel LS, Palmiter RD. Genetic identification of a neural circuit that suppresses appetite. Nature 2013; 503(7474): 111-4.

74. Wu Q, Clark MS, Palmiter RD. Deciphering a neuronal circuit thatmediates appetite. Nature 2012; 483: 594-8.

75. Li CS, Chung S, Lu DP, Cho YK. Descending projections from the nucleus accumbens shell suppress activity of taste-responsive neurons in the hamster parabrachial nuclei. J Neurophysiol 2012; 108(5): 1288-98.

76. Karimnamazi H, Travers SP, Travers JB. Oral and gastric input to the parabrachial nucleus of the rat. Brain Res 2002; 957(2): 193-206.

77. Cho YK, Li CS, Smith DV. Descending influences from the lateral hypothalamus and amygdala converge onto medullary taste neurons. Chem Senses 2003; 28(2): $155-71$.

78. Magableh A, Lundy R. Somatostatin and corticotrophin releasing hormone cell types are a major source of descending input from the forebrain to the parabrachial nucleus in mice. Chem Senses 2014; 39(8): 673-82.

79. Panguluri S, Saggu S, Lundy R. Comparison of somatostatin and corticotrophinreleasing hormone immunoreactivity in forebrain neurons projecting to tasteresponsive and non-responsive regions of the parabrachial nucleus in rat. Brain Res 2009; 1298: 57-69.

80. Coizet V, Dommett EJ, Klop EM, Redgrave P, Overton PG. The parabrachial nucleus is a critical link in the transmission of short latency nociceptive information to midbrain dopaminergic neurons. Neuroscience 2010; 168(1): 26372 . 
81. Freeman AS, Bunney BS. Activity of A9 and A10 dopaminergic neurons in unrestrained rats: further characterization and effects of apomorphine and cholecystokinin. Brain Res 1987; 405(1): 46-55.

82. Horvitz JC, Stewart T, Jacobs BL. Burst activity of ventral tegmental dopamine neurons is elicited by sensory stimuli in the awake cat. Brain Res 1997; 759(2): 251-8.

83. Lammel S, Lim BK, Malenka RC. Reward and aversion in a heterogeneous midbrain dopamine system. Neuropharmacology 2014; 76 Pt B: 351-9.

84. Bromberg-Martin ES, Matsumoto M, Hikosaka O. Dopamine in motivational control: rewarding, aversive, and alerting. Neuron 2010; 68(5): 815-34.

85. Schultz W. Multiple dopamine functions at different time courses. Annu Rev Neurosci 2007; 30: 259-88.

86. Ungless MA, Argilli E, Bonci A. Effects of stress and aversion on dopamine neurons: implications for addiction. Neurosci Biobehav Rev 2010; 35(2): 151-6.

87. Frank ST, Krumm B, Spanagel R. Cocaine-induced dopamine overflow within the nucleus accumbens measured by in vivo microdialysis: a meta-analysis. Synapse 2008; 62(4): 243-52.

88. Fiorino DF, Coury A, Fibiger HC, Phillips AG. Electrical stimulation of reward sites in the ventral tegmental area increases dopamine transmission in the nucleus accumbens of the rat. Behav Brain Res 1993; 55(2): 131-41.

89. Schultz W. Dopamine neurons and their role in reward mechanisms. Curr Opin Neurobiol 1997; 7(2): 191-7.

90. Ungless MA, Magill PJ, Bolam JP. Uniform inhibition of dopamine neurons in the ventral tegmental area by aversive stimuli. Science 2004; 303(5666): 2040-2.

91. D'Ardenne K, McClure SM, Nystrom LE, Cohen JD. BOLD responses reflecting dopaminergic signals in the human ventral tegmental area. Science 2008; 319(5867): 1264-7.

92. Cohen JY, Haesler S, Vong L, Lowell BB, Uchida N. Neuron-type-specific signals for reward and punishment in the ventral tegmental area. Nature 2012; 482(7383): 85-8.

93. Puopolo M, Raviola E, Bean BP. Roles of subthreshold calcium current and sodium current in spontaneous firing of mouse midbrain dopamine neurons. $J$ Neurosci 2007; 27(3): 645-56. 
94. Lammel S, Hetzel A, Hackel O, Jones I, Liss B, Roeper J. Unique properties of mesoprefrontal neurons within a dual mesocorticolimbic dopamine system. Neuron 2008; 57(5): 760-73.

95. Ding S, Wei W, Zhou FM. Molecular and functional differences in voltageactivated sodium currents between GABA projection neurons and dopamine neurons in the substantia nigra. J Neurophysiol 2011; 106(6): 3019-34.

96. Roeper J. Dissecting the diversity of midbrain dopamine neurons. Trends Neurosci 2013; 36(6): 336-42.

97. Berridge KC, Venier IL, Robinson TE. Taste reactivity analysis of 6hydroxydopamine-induced aphagia: implications for arousal and anhedonia hypotheses of dopamine function. Behav Neurosci 1989; 103(1): 36-45.

98. Schultz W. Behavioral theories and the neurophysiology of reward. Annu Rev Psychol 2006; 57: 87-115.

99. Cagniard B, Balsam PD, Brunner D, Zhuang X. Mice with chronically elevated dopamine exhibit enhanced motivation, but not learning, for a food reward. Neuropsychopharmacology 2006; 31(7): 1362-70.

100. Pecina S, Cagniard B, Berridge KC, Aldridge JW, Zhuang X. Hyperdopaminergic mutant mice have higher "wanting" but not "liking" for sweet rewards. J Neurosci 2003; 23(28): 9395-402.

101. Wyvell CL, Berridge KC. Intra-accumbens amphetamine increases the conditioned incentive salience of sucrose reward: enhancement of reward "wanting" without enhanced "liking" or response reinforcement. J Neurosci 2000; 20(21): 8122-30.

102. Tindell AJ, Berridge KC, Zhang J, Pecina S, Aldridge JW. Ventral pallidal neurons code incentive motivation: amplification by mesolimbic sensitization and amphetamine. Eur J Neurosci 2005; 22(10): 2617-34.

103. Berridge KC, Valenstein ES. What psychological process mediates feeding evoked by electrical stimulation of the lateral hypothalamus? Behav Neurosci 1991; 105(1): 3-14.

104. Sienkiewicz-Jarosz H, Scinska A, Kuran W, et al. Taste responses in patients with Parkinson's disease. J Neurol Neurosurg Psychiatry 2005; 76(1): 40-6.

105. Evans AH, Pavese N, Lawrence AD, et al. Compulsive drug use linked to sensitized ventral striatal dopamine transmission. Ann Neurol 2006; 59(5): 852-8. 
106. Dommett E, Coizet V, Blaha CD, et al. How visual stimuli activate dopaminergic neurons at short latency. Science 2005; 307(5714): 1476-9.

107. Horvitz JC. Dopamine gating of glutamatergic sensorimotor and incentive motivational input signals to the striatum. Behav Brain Res 2002; 137(1-2): 65 74.

108. Robbins TW, Everitt BJ. Functional studies of the central catecholamines. Int Rev Neurobiol 1982; 23: 303-65.

109. Salamone JD, Cousins MS, McCullough LD, Carriero DL, Berkowitz RJ. Nucleus accumbens dopamine release increases during instrumental lever pressing for food but not free food consumption. Pharmacol Biochem Behav 1994; 49(1): 25-31.

110. Stricker EM, Zigmond MJ. Brain monoamines, homeostasis, and adaptive behavior handbook of physiology: intrinsic regulatory systems of the brain. Bethesda (MD): American Physiological Society; 1986.

111. de la Fuente-Fernandez R, Phillips AG, Zamburlini M, et al. Dopamine release in human ventral striatum and expectation of reward. Behav Brain Res 2002; 136(2): 359-63.

112. Ito R, Dalley JW, Robbins TW, Everitt BJ. Dopamine release in the dorsal striatum during cocaine-seeking behavior under the control of a drug-associated cue. J Neurosci 2002; 22(14): 6247-53.

113. Phillips PE, Stuber GD, Heien ML, Wightman RM, Carelli RM. Subsecond dopamine release promotes cocaine seeking. Nature 2003; 422(6932): 614-8.

114. Tobler PN, O'Doherty J P, Dolan RJ, Schultz W. Human neural learning depends on reward prediction errors in the blocking paradigm. $J$ Neurophysiol 2006; 95(1): 301-10.

115. Datla KP, Ahier RG, Young AM, Gray JA, Joseph MH. Conditioned appetitive stimulus increases extracellular dopamine in the nucleus accumbens of the rat. Eur J Neurosci 2002; 16(10): 1987-93.

116. Blackburn JR, Phillips AG, Jakubovic A, Fibiger HC. Increased dopamine metabolism in the nucleus accumbens and striatum following consumption of a nutritive meal but not a palatable non-nutritive saccharin solution. Pharmacol Biochem Behav 1986; 25(5): 1095-100.

117. Fumero B, Fernandez-Vera JR, Gonzalez-Mora JL, Mas M. Changes in monoamine turnover in forebrain areas associated with masculine sexual behavior: a microdialysis study. Brain Res 1994; 662(1-2): 233-9. 
118. Kiyatkin EA, Gratton A. Electrochemical monitoring of extracellular dopamine in nucleus accumbens of rats lever-pressing for food. Brain Res 1994; 652(2): 22534.

119. Cannon CM, Abdallah L, Tecott LH, During MJ, Palmiter RD. Dysregulation of striatal dopamine signaling by amphetamine inhibits feeding by hungry mice. Neuron 2004; 44(3): 509-20.

120. Yin HH, Zhuang X, Balleine BW. Instrumental learning in hyperdopaminergic mice. Neurobiol Learn Mem 2006; 85(3): 283-8.

121. Gallistel CR, King A, McDonald R. Sources of variability and systematic error in mouse timing behavior. J Exp Psychol Anim Behav Process 2004; 30(1): 3-16.

122. Sanders AE, Cagniard B, Manning SN, Zhuang X. Dissecting aspects of motivation that are differentially modulated by dopamine and serotonin. Society for Neuroscience; 2003; Washington (DC); 2003.

123. Everitt BJ, Parkinson JA, Olmstead MC, Arroyo M, Robledo P, Robbins TW. Associative processes in addiction and reward. The role of amygdala-ventral striatal subsystems. Ann N Y Acad Sci 1999; 877: 412-38.

124. Robbins TW, Everitt BJ. Neurobehavioural mechanisms of reward and motivation. Curr Opin Neurobiol 1996; 6(2): 228-36.

125. Tindell AJ, Berridge KC, Aldridge JW. Ventral pallidal representation of pavlovian cues and reward: population and rate codes. J Neurosci 2004; 24(5): 1058-69.

126. Hamilton G. 2012.

127. Bassareo V, De Luca MA, Di Chiara G. Differential Expression of Motivational Stimulus Properties by Dopamine in Nucleus Accumbens Shell versus Core and Prefrontal Cortex. J Neurosci 2002; 22(11): 4709-19.

128. Thierry AM, Tassin JP, Blanc G, Glowinski J. Selective activation of mesocortical DA system by stress. Nature 1976; 263(5574): 242-4.

129. Herman JP, Guillonneau D, Dantzer R, Scatton B, Semerdjian-Rouquier L, Le Moal M. Differential effects of inescapable footshocks and of stimuli previously paired with inescapable footshocks on dopamine turnover in cortical and limbic areas of the rat. Life Sci 1982; 30(25): 2207-14. 
130. Abercrombie ED, Keefe KA, DiFrischia DS, Zigmond MJ. Differential effect of stress on in vivo dopamine release in striatum, nucleus accumbens, and medial frontal cortex. J Neurochem 1989; 52(5): 1655-8.

131. Doherty MD, Gratton A. High-speed chronoamperometric measurements of mesolimbic and nigrostriatal dopamine release associated with repeated daily stress. Brain Res 1992; 586(2): 295-302.

132. Doherty MD, Gratton A. Medial prefrontal cortical D1 receptor modulation of the meso-accumbens dopamine response to stress: an electrochemical study in freelybehaving rats. Brain Res 1996; 715(1-2): 86-97.

133. Sullivan RM, Gratton A. Relationships between stress-induced increases in medial prefrontal cortical dopamine and plasma corticosterone levels in rats: role of cerebral laterality. Neuroscience 1998; 83(1): 81-91.

134. Brake WG, Sullivan RM, Gratton A. Perinatal distress leads to lateralized medial prefrontal cortical dopamine hypofunction in adult rats. J Neurosci 2000; 20(14): $5538-43$.

135. Dunn AJ, File SE. Cold restraint alters dopamine metabolism in frontal cortex, nucleus accumbens and neostriatum. Physiol Behav 1983; 31(4): 511-3.

136. Claustre Y, Rivy JP, Dennis T, Scatton B. Pharmacological studies on stressinduced increase in frontal cortical dopamine metabolism in the rat. J Pharmacol Exp Ther 1986; 238(2): 693-700.

137. Imperato A, Puglisi-Allegra S, Casolini P, Zocchi A, Angelucci L. Stress-induced enhancement of dopamine and acetylcholine release in limbic structures: role of corticosterone. Eur J Pharmacol 1989; 165(2-3): 337-8.

138. Stevenson CW, Gratton A. Basolateral amygdala modulation of the nucleus accumbens dopamine response to stress: role of the medial prefrontal cortex. Eur J Neurosci 2003; 17(6): 1287-95.

139. Tidey JW, Miczek KA. Social defeat stress selectively alters mesocorticolimbic dopamine release: an in vivo microdialysis study. Brain Res 1996; 721(1-2): 1409.

140. Anstrom KK, Woodward DJ. Restraint increases dopaminergic burst firing in awake rats. Neuropsychopharmacology 2005; 30(10): 1832-40.

141. Berton O, McClung CA, Dileone RJ, et al. Essential role of BDNF in the mesolimbic dopamine pathway in social defeat stress. Science 2006; 311(5762): 864-8. 
142. Brischoux F, Chakraborty S, Brierley DI, Ungless MA. Phasic excitation of dopamine neurons in ventral VTA by noxious stimuli. Proc Natl Acad Sci U S A 2009; 106(12): 4894-9.

143. Polter AM, Kauer JA. Stress and VTA synapses: implications for addiction and depression. Eur J Neurosci 2014; 39(7): 1179-88.

144. Schultz W. Updating dopamine reward signals. Curr Opin Neurobiol 2013; 23(2): 229-38.

145. Tsai CT, Nakamura S, Iwama K. Inhibition of neuronal activity of the substantia nigra by noxious stimuli and its modification by the caudate nucleus. Brain Res 1980; 195(2): 299-311.

146. Schultz W, Romo R. Responses of nigrostriatal dopamine neurons to highintensity somatosensory stimulation in the anesthetized monkey. $J$ Neurophysiol 1987; 57(1): 201-17.

147. Brown MT, Henny P, Bolam JP, Magill PJ. Activity of neurochemically heterogeneous dopaminergic neurons in the substantia nigra during spontaneous and driven changes in brain state. J Neurosci 2009; 29(9): 2915-25.

148. Tan KR, Yvon C, Turiault M, et al. GABA neurons of the VTA drive conditioned place aversion. Neuron 2012; 73(6): 1173-83.

149. Mirenowicz J, Schultz W. Preferential activation of midbrain dopamine neurons by appetitive rather than aversive stimuli. Nature 1996; 379(6564): 449-51.

150. Joshua M, Adler A, Mitelman R, Vaadia E, Bergman H. Midbrain dopaminergic neurons and striatal cholinergic interneurons encode the difference between reward and aversive events at different epochs of probabilistic classical conditioning trials. J Neurosci 2008; 28(45): 11673-84.

151. Matsumoto M, Hikosaka O. Two types of dopamine neuron distinctly convey positive and negative motivational signals. Nature 2009; 459(7248): 837-41.

152. Mileykovskiy B, Morales M. Duration of inhibition of ventral tegmental area dopamine neurons encodes a level of conditioned fear. J Neurosci 2011; 31(20): 7471-6.

153. Young AM. Increased extracellular dopamine in nucleus accumbens in response to unconditioned and conditioned aversive stimuli: studies using $1 \mathrm{~min}$ microdialysis in rats. J Neurosci Methods 2004; 138(1-2): 57-63. 
154. Hommer DW, Bunney BS. Effect of sensory stimuli on the activity of dopaminergic neurons: involvement of non-dopaminergic nigral neurons and striato-nigral pathways. Life Sci 1980; 27(5): 377-86.

155. Guarraci FA, Kapp BS. An electrophysiological characterization of ventral tegmental area dopaminergic neurons during differential pavlovian fear conditioning in the awake rabbit. Behav Brain Res 1999; 99(2): 169-79.

156. Oades RD, Halliday GM. Ventral tegmental (A10) system: neurobiology. 1. Anatomy and connectivity. Brain Res 1987; 434(2): 117-65.

157. Yetnikoff L, Lavezzi HN, Reichard RA, Zahm DS. An update on the connections of the ventral mesencephalic dopaminergic complex. Neuroscience 2014; 282: 23 48.

158. Kauer JA, Malenka RC. Synaptic plasticity and addiction. Nat Rev Neurosci 2007; 8(11): 844-58.

159. Sesack SR, Grace AA. Cortico-Basal Ganglia reward network: microcircuitry. Neuropsychopharmacology 2010; 35(1): 27-47.

160. Huang AC, Shyu BC, Hsiao S, Chen TC, He AB. Neural substrates of fear conditioning, extinction, and spontaneous recovery in passive avoidance learning: a c-fos study in rats. Behav Brain Res 2013; 237: 23-31.

161. Kest K, Cruz I, Chen DH, Galaj E, Ranaldi R. A food-associated CS activates cFos in VTA DA neurons and elicits conditioned approach. Behav Brain Res 2012; 235(2): 150-7.

162. Majkutewicz I, Cecot T, Jerzemowska G, et al. Lesion of the ventral tegmental area amplifies stimulation-induced Fos expression in the rat brain. Brain Res 2010; 1320: 95-105.

163. McCabe BJ, Horn G. Learning-related changes in Fos-like immunoreactivity in the chick forebrain after imprinting. Proc Natl Acad Sci U S A 1994; 91(24): 11417-21.

164. Zhao-Shea R, Liu L, Soll LG, et al. Nicotine-mediated activation of dopaminergic neurons in distinct regions of the ventral tegmental area.

Neuropsychopharmacology 2011; 36(5): 1021-32.

165. Zhong J, Liang M, Akther S, Higashida C, Tsuji T, Higashida H. c-Fos expression in the paternal mouse brain induced by communicative interaction with maternal mates. Mol Brain 2014; 7: 66. 
166. Kaufling J, Veinante P, Pawlowski SA, Freund-Mercier MJ, Barrot M. Afferents to the GABAergic tail of the ventral tegmental area in the rat. J Comp Neurol 2009; 513(6): 597-621.

167. Ikemoto S. Dopamine reward circuitry: two projection systems from the ventral midbrain to the nucleus accumbens-olfactory tubercle complex. Brain Res Rev 2007; 56(1): 27-78.

168. Taber E. The cytoarchitecture of the brain stem of the cat. I. Brain stem nuclei of cat. J Comp Neurol 1961; 116: 27-69.

169. Phillipson OT. The cytoarchitecture of the interfascicular nucleus and ventral tegmental area of Tsai in the rat. J Comp Neurol 1979; 187(1): 85-98.

170. van Domburg PH, ten Donkelaar HJ. The human substantia nigra and ventral tegmental area. A neuroanatomical study with notes on aging and aging diseases. Adv Anat Embryol Cell Biol 1991; 121: 1-132.

171. Berman AC. The brain stem of the cat. A cytoarchitectonic atlas with stereotaxic coordinates. Madison (WI): Univ Wisconsin Press; 1968.

172. Swanson LW. The projections of the ventral tegmental area and adjacent regions: a combined fluorescent retrograde tracer and immunofluorescence study in the rat. Brain Res Bull 1982; 9(1-6): 321-53.

173. Ford CP, Mark GP, Williams JT. Properties and opioid inhibition of mesolimbic dopamine neurons vary according to target location. J Neurosci 2006; 26(10): 2788-97.

174. Margolis EB, Lock H, Chefer VI, Shippenberg TS, Hjelmstad GO, Fields HL. Kappa opioids selectively control dopaminergic neurons projecting to the prefrontal cortex. Proc Natl Acad Sci U S A 2006; 103(8): 2938-42.

175. Margolis EB, Mitchell JM, Ishikawa J, Hjelmstad GO, Fields HL. Midbrain dopamine neurons: projection target determines action potential duration and dopamine D(2) receptor inhibition. J Neurosci 2008; 28(36): 8908-13.

176. Lammel S, Ion DI, Roeper J, Malenka RC. Projection-specific modulation of dopamine neuron synapses by aversive and rewarding stimuli. Neuron 2011; 70(5): 855-62.

177. Lammel S, Lim BK, Ran C, et al. Input-specific control of reward and aversion in the ventral tegmental area. Nature 2012; 491(7423): 212-7.

178. Beckley JT, Evins CE, Fedarovich H, Gilstrap MJ, Woodward JJ. Medial prefrontal cortex inversely regulates toluene-induced changes in markers of 
synaptic plasticity of mesolimbic dopamine neurons. J Neurosci 2013; 33(2): 80413.

179. Hnasko TS, Hjelmstad GO, Fields HL, Edwards RH. Ventral tegmental area glutamate neurons: electrophysiological properties and projections. J Neurosci 2012; 32(43): 15076-85.

180. Ungless MA, Grace AA. Are you or aren't you? Challenges associated with physiologically identifying dopamine neurons. Trends Neurosci 2012; 35(7): 422 30 .

181. Zhang TA, Placzek AN, Dani JA. In vitro identification and electrophysiological characterization of dopamine neurons in the ventral tegmental area. Neuropharmacology 2010; 59(6): 431-6.

182. Perrotti LI, Bolanos CA, Choi KH, et al. DeltaFosB accumulates in a GABAergic cell population in the posterior tail of the ventral tegmental area after psychostimulant treatment. Eur J Neurosci 2005; 21(10): 2817-24.

183. Olson VG, Nestler EJ. Topographical organization of GABAergic neurons within the ventral tegmental area of the rat. Synapse 2007; 61(2): 87-95.

184. Li X, Qi J, Yamaguchi T, Wang HL, Morales M. Heterogeneous composition of dopamine neurons of the rat A10 region: molecular evidence for diverse signaling properties. Brain Struct Funct 2013; 218(5): 1159-76.

185. Tan Y, Williams ES, Zahm DS. Calbindin-D 28kD immunofluorescence in ventral mesencephalic neurons labeled following injections of Fluoro-Gold in nucleus accumbens subterritories: inverse relationship relative to known neurotoxin vulnerabilities. Brain Res 1999; 844(1-2): 67-77.

186. Barrot M, Calza L, Pozza M, Le Moal M, Piazza PV. Differential calbindinimmunoreactivity in dopamine neurons projecting to the rat striatal complex. Eur J Neurosci 2000; 12(12): 4578-82.

187. Gerfen CR, Baimbridge KG, Miller JJ. The neostriatal mosaic: compartmental distribution of calcium-binding protein and parvalbumin in the basal ganglia of the rat and monkey. Proc Natl Acad Sci U S A 1985; 82(24): 8780-4.

188. Geisler S, Zahm DS. Afferents of the ventral tegmental area in the rat-anatomical substratum for integrative functions. J Comp Neurol 2005; 490(3): 270-94.

189. Fadel J, Deutch AY. Anatomical substrates of orexin-dopamine interactions: lateral hypothalamic projections to the ventral tegmental area. Neuroscience 2002; 111(2): 379-87. 
190. Phillipson OT. Afferent projections to the ventral tegmental area of Tsai and interfascicular nucleus: a horseradish peroxidase study in the rat. J Comp Neurol 1979; 187(1): 117-43.

191. Geisler S, Derst C, Veh RW, Zahm DS. Glutamatergic afferents of the ventral tegmental area in the rat. J Neurosci 2007; 27(21): 5730-43.

192. Geisler S, Zahm DS. Neurotensin afferents of the ventral tegmental area in the rat: [1] re-examination of their origins and [2] responses to acute psychostimulant and antipsychotic drug administration. Eur J Neurosci 2006; 24(1): 116-34.

193. Zahm DS, Parsley KP, Schwartz ZM, Cheng AY. On lateral septum-like characteristics of outputs from the accumbal hedonic "hotspot" of Pecina and Berridge with commentary on the transitional nature of basal forebrain "boundaries". J Comp Neurol 2013; 521(1): 50-68.

194. Stamatakis AM, Jennings JH, Ung RL, et al. A unique population of ventral tegmental area neurons inhibits the lateral habenula to promote reward. Neuron 2013; 80(4): 1039-53.

195. Stamatakis AM, Stuber GD. Activation of lateral habenula inputs to the ventral midbrain promotes behavioral avoidance. Nat Neurosci 2012; 15(8): 1105-7.

196. Brinschwitz K, Dittgen A, Madai VI, Lommel R, Geisler S, Veh RW. Glutamatergic axons from the lateral habenula mainly terminate on GABAergic neurons of the ventral midbrain. Neuroscience 2010; 168(2): 463-76.

197. Jhou TC, Geisler S, Marinelli M, Degarmo BA, Zahm DS. The mesopontine rostromedial tegmental nucleus: A structure targeted by the lateral habenula that projects to the ventral tegmental area of Tsai and substantia nigra compacta. $J$ Comp Neurol 2009; 513(6): 566-96.

198. Taylor SR, Badurek S, Dileone RJ, Nashmi R, Minichiello L, Picciotto MR. GABAergic and glutamatergic efferents of the mouse ventral tegmental area. $J$ Comp Neurol 2014; 522(14): 3308-34.

199. Yamaguchi T, Sheen W, Morales M. Glutamatergic neurons are present in the rat ventral tegmental area. Eur J Neurosci 2007; 25(1): 106-18.

200. Yamaguchi T, Wang HL, Li X, Ng TH, Morales M. Mesocorticolimbic glutamatergic pathway. J Neurosci 2011; 31(23): 8476-90.

201. Nair-Roberts RG, Chatelain-Badie SD, Benson E, White-Cooper H, Bolam JP, Ungless MA. Stereological estimates of dopaminergic, GABAergic and glutamatergic neurons in the ventral tegmental area, substantia nigra and retrorubral field in the rat. Neuroscience 2008; 152(4): 1024-31. 
202. Beier KT, Steinberg EE, DeLoach KE, et al. Circuit Architecture of VTA Dopamine Neurons Revealed by Systematic Input-Output Mapping. Cell 2015; 162(3): 622-34.

203. van Zessen R, Phillips JL, Budygin EA, Stuber GD. Activation of VTA GABA neurons disrupts reward consumption. Neuron 2012; 73(6): 1184-94.

204. De Luca MA, Solinas M, Bimpisidis Z, Goldberg SR, Di Chiara G. Cannabinoid facilitation of behavioral and biochemical hedonic taste responses. Neuropharmacology 2012; 63(1): 161-8.

205. Berridge KC, Kringelbach ML. Pleasure systems in the brain. Neuron 2015; 86(3): 646-64.

206. Castro DC, Berridge KC. Opioid hedonic hotspot in nucleus accumbens shell: mu, delta, and kappa maps for enhancement of sweetness "liking" and "wanting". $J$ Neurosci 2014; 34(12): 4239-50.

207. Ho CY, Berridge KC. An orexin hotspot in ventral pallidum amplifies hedonic 'liking' for sweetness. Neuropsychopharmacology 2013; 38(9): 1655-64.

208. Pecina S, Berridge KC. Hedonic hot spot in nucleus accumbens shell: where do mu-opioids cause increased hedonic impact of sweetness? J Neurosci 2005; 25(50): 11777-86.

209. Smith KS, Berridge KC. The ventral pallidum and hedonic reward: neurochemical maps of sucrose "liking" and food intake. J Neurosci 2005; 25(38): 8637-49.

210. Castro DC, Cole SL, Berridge KC. Lateral hypothalamus, nucleus accumbens, and ventral pallidum roles in eating and hunger: interactions between homeostatic and reward circuitry. Front Syst Neurosci 2015; 9: 90.

211. Shimura T, Kamada Y, Yamamoto T. Ventral tegmental lesions reduce overconsumption of normally preferred taste fluid in rats. Behav Brain Res 2002; 134(1-2): 123-30.

212. Park TH, Carr KD. Neuroanatomical patterns of fos-like immunoreactivity induced by a palatable meal and meal-paired environment in saline- and naltrexone-treated rats. Brain Res 1998; 805(1-2): 169-80.

213. Geran LC, Travers SP. Bitter-responsive gustatory neurons in the rat parabrachial nucleus. J Neurophysiol 2009; 101(3): 1598-612. 
214. Halsell CB, Travers SP. Anterior and posterior oral cavity responsive neurons are differentially distributed among parabrachial subnuclei in rat. J Neurophysiol 1997; 78(2): 920-38.

215. King CT, Deyrup LD, Dodson SE, Galvin KE, Garcea M, Spector AC. Effects of gustatory nerve transection and regeneration on quinine-stimulated Fos-like immunoreactivity in the parabrachial nucleus of the rat. J Comp Neurol 2003; 465(2): 296-308.

216. Hajnal A, Norgren R. Taste pathways that mediate accumbens dopamine release by sapid sucrose. Physiol Behav 2005; 84(3): 363-9.

217. Saites LN, Tokita K, Lu L, Boughter JD, Jr. Parabrachial nucleus projections to ventral tegmental area transmit taste information to the reward system in mice. Association for Chemoreception Sciences; 2015; Bonita Springs (FL); 2015.

218. Saites LN, Lu L, Boughter JD, Jr. Ventral tegmental area afferents for tasterelated inputs with both positive and negative hedonic value. Association for Chemoreception Sciences; 2016; Bonita Springs (FL); 2016.

219. Maeda H, Mogenson GJ. Electrophysiological responses of neurons of the ventral tegmental area to electrical stimulation of amygdala and lateral septum.

Neuroscience 1981; 6(3): 367-76.

220. Gonzales C, Chesselet MF. Amygdalonigral pathway: an anterograde study in the rat with Phaseolus vulgaris leucoagglutinin (PHA-L). J Comp Neurol 1990; 297(2): 182-200.

221. Wallace DM, Magnuson DJ, Gray TS. Organization of amygdaloid projections to brainstem dopaminergic, noradrenergic, and adrenergic cell groups in the rat. Brain Res Bull 1992; 28(3): 447-54.

222. Bittencourt JC, Presse F, Arias C, et al. The melanin-concentrating hormone system of the rat brain: an immuno- and hybridization histochemical characterization. J Comp Neurol 1992; 319(2): 218-45.

223. Peyron C, Tighe DK, van den Pol AN, et al. Neurons containing hypocretin (orexin) project to multiple neuronal systems. J Neurosci 1998; 18(23): 999610015.

224. Barson JR, Morganstern I, Leibowitz SF. Complementary roles of orexin and melanin-concentrating hormone in feeding behavior. Int J Endocrinol 2013; 2013: 983964.

225. Millhouse OE, De Olmos J. The amygdaloid complex. In: Ben-Ari Y, ed. Aspects of the neuronal organization of the amygdala. Amsterdam: Elsevier; 1981: 33-43. 
226. Pitkanen A. Connectivity of the rat amygdaloid complex. In: Aggleton JP, ed. The amygdala: A functional analysis 2nd edition. Oxford (UK): Oxford University Press; 2000: 31-115.

227. Samuelsen CL, Gardner MP, Fontanini A. Thalamic contribution to cortical processing of taste and expectation. $J$ Neurosci 2013; 33(5): 1815-27.

228. Fontanini A, Grossman SE, Figueroa JA, Katz DB. Distinct subtypes of basolateral amygdala taste neurons reflect palatability and reward. $J$ Neurosci 2009; 29(8): 2486-95.

229. Samuelsen CL, Gardner MP, Fontanini A. Effects of cue-triggered expectation on cortical processing of taste. Neuron 2012; 74(2): 410-22.

230. Ogawa H, Nomura T. Receptive field properties of thalamo-cortical taste relay neurons in the parvicellular part of the posteromedial ventral nucleus in rats. Exp Brain Res 1988; 73(2): 364-70.

231. Verhagen JV, Giza BK, Scott TR. Responses to taste stimulation in the ventroposteromedial nucleus of the thalamus in rats. J Neurophysiol 2003; 89(1): $265-75$.

232. Mansour A, Khachaturian H, Lewis ME, Akil H, Watson SJ. Autoradiographic differentiation of mu, delta, and kappa opioid receptors in the rat forebrain and midbrain. J Neurosci 1987; 7(8): 2445-64.

233. Carr KD, Aleman DO, Bak TH, Simon EJ. Effects of parabrachial opioid antagonism on stimulation-induced feeding. Brain Res 1991; 545(1-2): 283-6.

234. Moufid-Bellancourt S, Velley L. Effects of morphine injection into the parabrachial area on saccharin preference: modulation by lateral hypothalamic neurons. Pharmacol Biochem Behav 1994; 48(1): 127-33.

235. Shimura T, Watanabe U, Yanagawa Y, Yamamoto T. Altered taste function in mice deficient in the 65-kDa isoform of glutamate decarboxylase. Neurosci Lett 2004; 356(3): 171-4.

236. Cooper SJ, van der Hoek G, Kirkham TC. Bi-directional changes in sham feeding in the rat produced by benzodiazepine receptor ligands. Physiol Behav 1988; 42(3): 211-6.

237. Higgs S, Cooper SJ. Hyperphagia induced by direct administration of midazolam into the parabrachial nucleus of the rat. Eur J Pharmacol 1996; 313(1-2): 1-9. 
238. Soderpalm AH, Berridge KC. The hedonic impact and intake of food are increased by midazolam microinjection in the parabrachial nucleus. Brain Res 2000; 877(2): 288-97.

239. De Luca MA. Habituation of the responsiveness of mesolimbic and mesocortical dopamine transmission to taste stimuli. Front Integr Neurosci 2014; 8: 21.

240. Tellez LA, Han W, Zhang X, et al. Separate circuitries encode the hedonic and nutritional values of sugar. Nat Neurosci 2016; 19(3): 465-70.

241. Boekhoudt L, Roelofs TJM, de Jong JW, et al. Does activation of midbrain dopamine neurons promote or reduce feeding? Int J Obes (Lond) 2017; 41(7): $1131-40$.

242. Zagon A, Totterdell S, Jones RS. Direct projections from the ventrolateral medulla oblongata to the limbic forebrain: anterograde and retrograde tracttracing studies in the rat. J Comp Neurol 1994; 340(4): 445-68.

243. Hajnal A, Norgren R. Dopamine release by sucrose. The senses: A comprehensive reference; 2008: 459-68.

244. Zhang Y, Hoon MA, Chandrashekar J, et al. Coding of sweet, bitter, and umami tastes: different receptor cells sharing similar signaling pathways. Cell 2003; 112(3): 293-301.

245. Damak S, Rong M, Yasumatsu K, et al. Trpm5 null mice respond to bitter, sweet, and umami compounds. Chem Senses 2006; 31(3): 253-64.

246. de Araujo IE, Oliveira-Maia AJ, Sotnikova TD, et al. Food reward in the absence of taste receptor signaling. Neuron 2008; 57(6): 930-41.

247. Gosnell BA, Levine AS. Stimulation of ingestive behavior by preferential and selective opioid agonists. In: Cooper SJ, Clifton PG, eds. Drug receptor subtypes and ingestive behavior. New York: Academic Press; 1996: 147-66.

248. Bozarth MA, Wise RA. Intracranial self-administration of morphine into the ventral tegmental area in rats. Life Sci 1981; 28(5): 551-5.

249. Goeders NE, Smith JE, Lane JD. Intracranial self-administration of the endocoid methionine-enkephalin. J Am Osteopath Assoc 1984; 84(1 Suppl): 148-51.

250. Kirkham TC, Cooper SJ. Naloxone attenuation of sham feeding is modified by manipulation of sucrose concentration. Physiol Behav 1998; 44: 491-4. 
251. Echo JA, Lamonte N, Ackerman TF, Bodnar RJ. Alterations in food intake elicited by GABA and opioid agonists and antagonists administered into the ventral tegmental area region of rats. Physiol Behav 2002; 76(1): 107-16.

252. Khaimova E, Kandov Y, Israel Y, Cataldo G, Hadjimarkou MM, Bodnar RJ. Opioid receptor subtype antagonists differentially alter GABA agonist-induced feeding elicited from either the nucleus accumbens shell or ventral tegmental area regions in rats. Brain Res 2004; 1026(2): 284-94.

253. Murschall A, Hauber W. Inactivation of the ventral tegmental area abolished the general excitatory influence of Pavlovian cues on instrumental performance. Learn Mem 2006; 13(2): 123-6.

254. Maliszewska-Scislo M, Trojniar W. Effect of unilateral injection of MK-801 into the area of A10 cells on feeding evoked by stimulation of homologous area in the contralateral hemisphere. Acta Neurobiol Exp (Wars) 2000; 60(4): 489-94.

255. Baird JP, Choe A, Loveland JL, et al. Orexin-A hyperphagia: hindbrain participation in consummatory feeding responses. Endocrinology 2009; 150(3): $1202-16$.

256. Baird JP, Rios C, Loveland JL, Beck J, Tran A, Mahoney CE. Effects of hindbrain melanin-concentrating hormone and neuropeptide $\mathrm{Y}$ administration on licking for water, saccharin, and sucrose solutions. Am J Physiol Regul Integr Comp Physiol 2008; 294(2): R329-43.

257. DiNardo LA, Travers JB. Distribution of fos-like immunoreactivity in the medullary reticular formation of the rat after gustatory elicited ingestion and rejection behaviors. J Neurosci 1997; 17(10): 3826-39.

258. Harrer MI, Travers SP. Topographic organization of Fos-like immunoreactivity in the rostral nucleus of the solitary tract evoked by gustatory stimulation with sucrose and quinine. Brain Res 1996; 711(1-2): 125-37.

259. Saites LN, Goldsmith Z, Densky J, Guedes VA, Boughter JD, Jr. Mice perceive synergistic umami mixtures as tasting sweet. Chem Senses 2015; 40(5): 295-303.

260. Savchenko VL, Boughter JD, Jr. Regulation of neuronal activation by Alpha2A adrenergic receptor agonist. Neurotox Res 2011; 20(3): 226-39.

261. Travers JB, Herman K, Yoo J, Travers SP. Taste reactivity and Fos expression in GAD1-EGFP transgenic mice. Chem Senses 2007; 32(2): 129-37.

262. Zhang YH, Ennis M. Inactivation of the periaqueductal gray attenuates antinociception elicited by stimulation of the rat medial preoptic area. Neurosci Lett 2007; 429(2-3): 105-10. 
263. Kovacs KJ. Measurement of immediate-early gene activation- c-fos and beyond. $J$ Neuroendocrinol 2008; 20(6): 665-72.

264. Hashimoto K, Obata K, Ogawa H. Characterization of parabrachial subnuclei in mice with regard to salt tastants: possible independence of taste relay from visceral processing. Chem Senses 2009; 34(3): 253-67.

265. Paxinos G, Franklin KB. The mouse brain in stereotaxic coordinates. Amsterdam: Elsevier; 2001.

266. Science AIfB. Allen Brain Atlas. 2015. http://www.brain-map.org/.

267. Yee KK, Sukumaran SK, Kotha R, Gilbertson TA, Margolskee RF. Glucose transporters and ATP-gated K+ (KATP) metabolic sensors are present in type 1 taste receptor 3 (T1r3)-expressing taste cells. Proc Natl Acad Sci U S A 2011; 108(13): 5431-6.

268. Sukumaran SK, Yee KK, Iwata S, et al. Taste cell-expressed alpha-glucosidase enzymes contribute to gustatory responses to disaccharides. Proc Natl Acad Sci U $S$ A 2016; 113(21): 6035-40.

269. Sewards TV. Dual separate pathways for sensory and hedonic aspects of taste. Brain Res Bull 2004; 62(4): 271-83.

270. McCullough KM, Morrison FG, Ressler KJ. Bridging the Gap: Towards a celltype specific understanding of neural circuits underlying fear behaviors. Neurobiol Learn Mem 2016; 135: 27-39.

271. Fu Y, Yuan Y, Halliday G, Rusznak Z, Watson C, Paxinos G. A cytoarchitectonic and chemoarchitectonic analysis of the dopamine cell groups in the substantia nigra, ventral tegmental area, and retrorubral field in the mouse. Brain Struct Funct 2012; 217(2): 591-612.

272. Waraczynski M, Perkins M. Temporary inactivation of the retrorubral fields decreases the rewarding effect of medial forebrain bundle stimulation. Brain Res 2000; 885(2): 154-65.

273. Tamamaki N, Yanagawa Y, Tomioka R, Miyazaki J, Obata K, Kaneko T. Green fluorescent protein expression and colocalization with calretinin, parvalbumin, and somatostatin in the GAD67-GFP knock-in mouse. J Comp Neurol 2003; 467(1): 60-79.

274. Lazarus MS, Krishnan K, Huang ZJ. GAD67 deficiency in parvalbumin interneurons produces deficits in inhibitory transmission and network disinhibition in mouse prefrontal cortex. Cereb Cortex 2015; 25(5): 1290-6. 
275. Mungarndee SS, Lundy RF, Jr., Norgren R. Expression of Fos during sham sucrose intake in rats with central gustatory lesions. Am J Physiol Regul Integr Comp Physiol 2008; 295(3): R751-63.

276. Flores JA, Galan-Rodriguez B, Ramiro-Fuentes S, Fernandez-Espejo E. Role for dopamine neurons of the rostral linear nucleus and periaqueductal gray in the rewarding and sensitizing properties of heroin. Neuropsychopharmacology 2006; 31(7): 1475-88.

277. Boender AJ, de Jong JW, Boekhoudt L, Luijendijk MC, van der Plasse G, Adan RA. Combined use of the canine adenovirus-2 and DREADD-technology to activate specific neural pathways in vivo. PLoS One 2014; 9(4): e95392.

278. Higgs S, Cooper SJ. Evidence for early opioid modulation of licking responses to sucrose and intralipid: a microstructural analysis in the rat. Psychopharmacology (Berl) 1998; 139(4): 342-55.

279. Pittman DW, McGinnis MR, Richardson LM, Miller EJ, Alimohamed ML, Baird JP. Multiple processes underlie benzodiazepine-mediated increases in the consumption of accepted and avoided stimuli. Chem Senses 2012; 37(5): 431-44.

280. Wilson JD, Nicklous DM, Aloyo VJ, Simansky KJ. An orexigenic role for muopioid receptors in the lateral parabrachial nucleus. Am J Physiol Regul Integr Comp Physiol 2003; 285(5): R1055-65.

281. St John SJ, Lu L, Williams RW, Saputra J, Boughter JD, Jr. Genetic control of oromotor phenotypes: A survey of licking and ingestive behaviors in highly diverse strains of mice. Physiol Behav 2017; 177: 34-43.

282. Armbruster BN, Li X, Pausch MH, Herlitze S, Roth BL. Evolving the lock to fit the key to create a family of $\mathrm{G}$ protein-coupled receptors potently activated by an inert ligand. Proc Natl Acad Sci U S A 2007; 104(12): 5163-8.

283. Krashes MJ, Koda S, Ye C, et al. Rapid, reversible activation of AgRP neurons drives feeding behavior in mice. J Clin Invest 2011; 121(4): 1424-8.

284. Rothermel M, Brunert D, Zabawa C, Diaz-Quesada M, Wachowiak M. Transgene expression in target-defined neuron populations mediated by retrograde infection with adeno-associated viral vectors. J Neurosci 2013; 33(38): 15195-206.

285. Boughter JD, Jr., St John SJ, Noel DT, Ndubuizu O, Smith DV. A brief-access test for bitter taste in mice. Chem Senses 2002; 27(2): 133-42.

286. St John SJ, Boughter JD, Jr. Orosensory responsiveness to and preference for hydroxide-containing salts in mice. Chem Senses 2009; 34(6): 487-98. 
287. Treesukosol Y, Bi S, Moran TH. Overexpression of neuropeptide Y in the dorsomedial hypothalamus increases trial initiation but does not significantly alter concentration-dependent licking to sucrose in a brief-access taste test. Physiol Behav 2013; 110-111: 109-14.

288. Roth BL. DREADDs for Neuroscientists. Neuron 2016; 89(4): 683-94.

289. Wahlsten D, Rustay NR, Metten P, Crabbe JC. In search of a better mouse test. Trends Neurosci 2003; 26(3): 132-6.

290. Loos M, Koopmans B, Aarts E, et al. Within-strain variation in behavior differs consistently between common inbred strains of mice. Mamm Genome 2015; 26(78): 348-54.

291. Gonzalez-Hernandez T, Barroso-Chinea P, Acevedo A, Salido E, Rodriguez M. Colocalization of tyrosine hydroxylase and GAD65 mRNA in mesostriatal neurons. Eur J Neurosci 2001; 13(1): 57-67.

292. Oliveira-Maia AJ, de Araujo IE, Monteiro C, Workman V, Galhardo V, Nicolelis MA. The insular cortex controls food preferences independently of taste receptor signaling. Front Syst Neurosci 2012; 6: 5.

293. de Araujo IE. Sweet taste signaling and the formation of memories of energy sources. Front Syst Neurosci 2011; 5: 99.

294. King MS. Distribution of Fos-immunoreactive neurons in the gustatory cortex elicited by intra-oral infusion of taste solutions in conscious rats. Brain Res 2018; 1683: $67-77$.

295. Dragunow M, Faull R. The use of c-fos as a metabolic marker in neuronal pathway tracing. J Neurosci Methods 1989; 29(3): 261-5.

296. Kobayashi M, Cui Y, Sako T, et al. Functional neuroimaging of aversive tasterelated areas in the alert rat revealed by positron emission tomography. J Neurosci Res 2013; 91(10): 1363-70.

297. Yamamoto T. Central mechanisms of taste: Cognition, emotion and taste-elicited behaviors. Japanese Dental Science Review 2008; 44(2): 91-9.

298. Anand BK, Brobeck JR. Localization of a "feeding center" in the hypothalamus of the rat. Proc Soc Exp Biol Med 1951; 77(2): 323-4.

299. Delgado JM, Anand BK. Increase of food intake induced by electrical stimulation of the lateral hypothalamus. Am J Physiol 1953; 172(1): 162-8. 
300. Harris GC, Wimmer M, Aston-Jones G. A role for lateral hypothalamic orexin neurons in reward seeking. Nature 2005; 437(7058): 556-9.

301. Nishikawa T, Fage D, Scatton B. Evidence for, and nature of, the tonic inhibitory influence of habenulointerpeduncular pathways upon cerebral dopaminergic transmission in the rat. Brain Res 1986; 373(1-2): 324-36.

302. Herkenham M, Nauta WJ. Afferent connections of the habenular nuclei in the rat. A horseradish peroxidase study, with a note on the fiber-of-passage problem. $J$ Comp Neurol 1977; 173(1): 123-46.

303. Herkenham M, Nauta WJ. Efferent connections of the habenular nuclei in the rat. J Comp Neurol 1979; 187(1): 19-47.

304. Selverston AI. Invertebrate central pattern generator circuits. Philos Trans $R$ Soc Lond B Biol Sci 2010; 365(1551): 2329-45.

305. Aldridge JW, Berridge $\mathrm{KC}$, Rosen AR. Basal ganglia neural mechanisms of natural movement sequences. Can J Physiol Pharmacol 2004; 82(8-9): 732-9.

306. Miller P, Katz DB. Stochastic transitions between neural states in taste processing and decision-making. J Neurosci 2010; 30(7): 2559-70.

307. Sadacca BF, Mukherjee N, Vladusich T, Li JX, Katz DB, Miller P. The Behavioral Relevance of Cortical Neural Ensemble Responses Emerges Suddenly. J Neurosci 2016; 36(3): 655-69.

308. Goncalves L, Sego C, Metzger M. Differential projections from the lateral habenula to the rostromedial tegmental nucleus and ventral tegmental area in the rat. J Comp Neurol 2012; 520(6): 1278-300.

309. Scott TR. Learning through the taste system. Front Syst Neurosci 2011; 5: 87.

310. Garcia J, Kimeldorf DJ, Koelling RA. Conditioned aversion to saccharin resulting from exposure to gamma radiation. Science 1955; 122(3160): 157-8.

311. Garcia J, Ervin FR. Appetites, aversions, and addictions: a model for visceral memory. Recent Adv Biol Psychiatry 1968; 10: 284-93.

312. Reilly S, Schachtman TR. Conditioned taste aversion : behavioral and neural processes. Oxford ; New York: Oxford University Press; 2009.

313. Sclafani A. Oral and postoral determinants of food reward. Physiol Behav 2004; 81(5): 773-9. 
314. Giza BK, Ackroff K, McCaughey SA, Sclafani A, Scott TR. Preference conditioning alters taste responses in the nucleus of the solitary tract of the rat. Am J Physiol 1997; 273(4 Pt 2): R1230-40.

315. Elizade G, Sclafani A. Flavor preferences conditioned by intragastric polycose infusions: A detailed analysis using an electronic esophagus preparation. Physiol Behav 1990; 47: 63-77.

316. Fanselow MS, Birk J. Flavor-flavor associations induce hedonic shifts in taste preference. Animal Learning \& Behavior 1982; 10(2): 223-8.

317. Yamamoto T, Fujimoto Y, Shimura T, Sakai N. Conditioned taste aversion in rats with excitotoxic brain lesions. Neurosci Res 1995; 22(1): 31-49.

318. Petrovich GD, Ross CA, Mody P, Holland PC, Gallagher M. Central, but not basolateral, amygdala is critical for control of feeding by aversive learned cues. $J$ Neurosci 2009; 29(48): 15205-12.

319. Aguero A, Arnedo M, Gallo M, Puerto A. The functional relevance of the lateral parabrachial nucleus in lithium chloride-induced aversion learning. Pharmacol Biochem Behav 1993; 45(4): 973-8.

320. Sakai N, Yamamoto T. Role of the medial and lateral parabrachial nucleus in acquisition and retention of conditioned taste aversion in rats. Behav Brain Res 1998; 93(1-2): 63-70.

321. Reilly S. The parabrachial nucleus and conditioned taste aversion. Brain Res Bull 1999; 48(3): 239-54.

322. Reilly S, Trifunovic R. Lateral parabrachial nucleus lesions in the rat: aversive and appetitive gustatory conditioning. Brain Res Bull 2000; 52(4): 269-78.

323. Spector AC, Norgren R, Grill HJ. Parabrachial gustatory lesions impair taste aversion learning in rats. Behav Neurosci 1992; 106(1): 147-61.

324. Rotella FM, Vig V, Olsson K, et al. Baclofen differentially mediates fructoseconditioned flavor preference and quinine-conditioned flavor avoidance in rats. Eur J Pharmacol 2016; 775: 15-21.

325. Korotkova TM, Ponomarenko AA, Brown RE, Haas HL. Functional diversity of ventral midbrain dopamine and GABAergic neurons. Mol Neurobiol 2004; 29(3): $243-59$.

326. Luscher C, Malenka RC. Drug-evoked synaptic plasticity in addiction: from molecular changes to circuit remodeling. Neuron 2011; 69(4): 650-63. 
327. Mogenson GJ, Swanson LW, Wu M. Neural projections from nucleus accumbens to globus pallidus, substantia innominata, and lateral preoptic-lateral hypothalamic area: an anatomical and electrophysiological investigation in the rat. J Neurosci 1983; 3(1): 189-202.

328. Zahm DS, Heimer L. Two transpallidal pathways originating in the rat nucleus accumbens. J Comp Neurol 1990; 302(3): 437-46.

329. Heimer L, Zahm DS, Churchill L, Kalivas PW, Wohltmann C. Specificity in the projection patterns of accumbal core and shell in the rat. Neuroscience 1991; 41(1): 89-125.

330. Lu XY, Ghasemzadeh MB, Kalivas PW. Expression of D1 receptor, D2 receptor, substance $\mathrm{P}$ and enkephalin messenger RNAs in the neurons projecting from the nucleus accumbens. Neuroscience 1998; 82(3): 767-80.

331. Usuda I, Tanaka K, Chiba T. Efferent projections of the nucleus accumbens in the rat with special reference to subdivision of the nucleus: biotinylated dextran amine study. Brain Res 1998; 797(1): 73-93.

332. Zhou L, Furuta T, Kaneko T. Chemical organization of projection neurons in the rat accumbens nucleus and olfactory tubercle. Neuroscience 2003; 120(3): 78398.

333. Humphries MD, Prescott TJ. The ventral basal ganglia, a selection mechanism at the crossroads of space, strategy, and reward. Prog Neurobiol 2010; 90(4): 385417. 


\section{VITA}

Louis "Nick" Saites was born in Ann Arbor, Michigan in 1986, but from age 7, he grew up in the small, rural town of Lewisburg, Tennessee. On reaching adulthood, he eventually moved to Murfreesboro, Tennessee to earn his Bachelor of Science in Biology at Middle Tennessee State University. He entered the Neuroscience track of the Integrated Biomedical Sciences program at University of Tennessee Health Science Center in Memphis, Tennessee in August of 2012. Outside the lab, he also dedicated a significant amount of time volunteering as a meeting facilitator for SMART Recovery, a science-based, self-help program for individuals overcoming addictive behavior. He served as President of the Graduate Student Executive Council during the 2013-2014 academic year and in several other campus leadership positions throughout his graduate career. He published his first paper in June 2015 in the journal Chemical Senses and has presented 4 posters at conferences of the Association for Chemoreception Sciences (AChemS). He earned his Ph.D. in August of 2018. 